NBSIR 76-1179

\title{
Attack and Release Characteristics of Compression Hearing Aids
}

Edwin D. Burnett and Martin A. Bassin

Institute for Basic Standards

National Bureau of Standards

Washington, D. C. 20234

December 1976

Final

Prepared for

Department of Medicine and Surgery

Veterans Administration

Washington, D. C. 20422 



\section{ATTACK AND RELEASE \\ CHARACTERISTICS OF COMPRESSION HEARING AIDS}

Edwin D. Burnett and Martin A. Bassin

Institute for Basic Standards

National Bureau of Standards

Washngton, D. C. 20234

December 1976

Final

Prepared for

Department of Medicine and Surgery

Veterans Administration

Washington, D. C. 20422

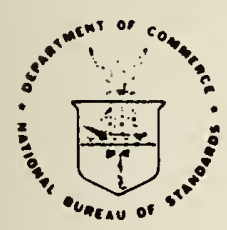

U.S. DEPARTMENT OF COMMERCE, Elliot L. Richardson, Secretary Edward O. Vetter, Under Secretany

Dr. Betsy Ancker-Johnson, Assistant Secretary for Science and Technology

NATIONAL BUREAU OF STANDARDS, Emest Ambler, Acting Director 

Edwin D. Burnett and Martin A. Bassin

Institute for Basic Standards National Bureau of Standards

Washington, D.C. 20234

\section{ABSTRACT}

The attack and release time characteristics of 81 compression hearing aids are presented. The input signal used was that specified in the standard, ANSI S3.22. 



\section{INTRODUCTION}

The attack and release time characteristics of compression hearing aids were recorded by NBS and incorporated for the first time as part of the regular testing of FY-76 hearing aids for the Veterans Administration. This report describes the method used to obtain the results and presents oscilloscope recordings of the envelopes of the attack and release intervals. The actual attack and release times can be obtalned from these recordings using the definitions given in Figure 1.

\section{METHOD}

To determine the attack and release times of hearing aids tested by NBS the envelope input signal shown in the top of Figure 1 is used. This is the signal specified in the standard, ANSI S3.22 (1). The carrier frequency of this signal is $2 \mathrm{kHz}$. The amplitude of the input signal corresponds to a sound pressure level (SPL) of elther 55 or $80 \mathrm{~dB}$ re $20 \mu \mathrm{Pa}$. To determine the attack time of the hearing aid the SPL is increased abruptly from a SPL of $55 \mathrm{~dB}$ to one of $80 \mathrm{~dB}$. The release time is determined by abruptly reducing the SPL from $80 \mathrm{~dB}$ to $55 \mathrm{~dB}$. An idealized envelope of the output signal from the hearing aid is shown in the bottom of Figure 1 . A1though one burst of the $2 \mathrm{kHz}$ signal shown in the top of Figure 1 would be sufficient for the measurement, in practice it is convenient to have this signal repeat itself a sufficient number of times so that adjustments to signal level into the oscilloscope can be made prior to taking the permanent recording of the output signal. The duration of the signal at each of the two SPLs is not critical; however, it must be somewhat longer than the time required for the hearing aid output to reach steady-state conditions after 
the level is changed. A duration of $200 \mathrm{~ms}$ for each of the signal levels has been found sufficient for most hearing aids.

To minimize the occurrence of spurious transients in the input signal envelope, the abrupt changes in the levels are made at a time corresponding to a zero voltage crossing of the input carrier signal.

The test is performed in a free field. The hearing aid volume control is turned to its maximum setting and its receiver is coupled to a Zwislocki coupler having a type HA-2 adaptor. This adaptor is used even for high-pass hearing aids which are normally tested for their other characteristics on an open couplér.

The coupler's microphone output voltage is displayed on a large-screen $(15 \times 20 \mathrm{~cm})$ oscilloscope. A total sweep time of 0.5 seconds is used so that the entire duration of the high and low amplitude portions of the signal are displayed. The vertical amplitude control on the oscilloscope is adjusted so the initial overshoot during the attack portion of the signal goes beyond maximum limits of the screen. This permits one to obtain a recording with higher amplitude resolution for those portions of the signal that are used to determine the attack and release times. A second oscilloscope recording is made usually with a total sweep time of 0.05 seconds, with the amplitude attenuated such that the entire overshoot region can be seen.

On each of the figures depicting the envelope of the output signal of the hearing aid are three labels. The first label indicates the alphanumeric code corresponding to hearing aid manufacturer and model number which are given in Table 1. The second label denotes the total duration in seconds 
of the sweep of the horizontal axis. This axis is linear so that half the length of the axis corresponds to half the total duration, and so on. The third label indicates the amount of attenuation of the signal relative to that used to obtain the preceding record of the attack and release portions of the same hearing aid. For some of the hearing aids a number is shown above the high-leve1 portion of the signal. This number is the steadystate output SPL at $2 \mathrm{kHz}$ for an input SPL of $80 \mathrm{~dB}$.

Figures 2 and 3 show the output of the "1/2 inch" reference microphone. The minor irregularities in the steady-state portion of the signal are caused by low frequency acoustic noise. These components do not affect the test results since they fall below the frequency region passed by the hearing aids. Figure 3 shows that a time interval of $3 \mathrm{msec}$ ( 6 cycles of the $2-\mathrm{kHz}$ signal) is required for the acoustical signal to reach steady-state conditions. The peak value of the first cycle of the high amplitude portion of the signal is approximately $1.75 \mathrm{~dB}$ below the steady-state peak value.

\section{REFERENCE}

1. ANSI S3.22 (1976), "Specification of Hearing Aid Characteristics," American National Standards Institute, New York, N.Y. 
Hearing Aid Codes, Manufacturers, and Models

Code
AT 310-AT 312
DA2 17-DA2 19
DX145-DX147
DX148-DX150
DY001-DY003
FI229-FI231
FI232-FI234
FI238-FI240
$\mathrm{HCO} 19-\mathrm{HCO} 21$
$\mathrm{HCO} 22-\mathrm{HCO} 24$
$\mathrm{HCO} 25-\mathrm{HCO} 27$
LE127-LE129
N0166-N0168
N0172-N0174
OI214-OI 216
QU259-QU261
RA256-RA258
SI157-SI 159
ST004-ST007
TE 301-TE303
UN001-UN003
UN007-UN009
UN013-UN015
UN019-UN021
WI 109-WI111
WI 121-WI 123
ZE307-ZE 310

Manufacturer

Audiotone
Dahlberg
Danavox
Danavox
Dynatone
Fidelity
Fidelity
Fidelity
HC Electronics
HC Electronics
HC Electronics
Lehr
Norelco
Norelco
Oticon
Qualitone
Radioear
Siemens
Starkey
Telex
Unitron
Unitron
Viennatone
Viennatone
Widex
Widex
Zenith

Model

A24 Compression HT1233 Compression

745 AGC

745 DAGC

$500 \mathrm{P}$

F34

F52Bicros

F58D

HC527LW

HC527SEW

HC801AGC

Star 6DRC

HP 8234AGC

HP 8274 Lim, Time, TC

E14C

$\mathrm{CNE}$

1063

24PP-AGC-I

4112

336DC

295T

$695 \mathrm{H}$

88AMA-D

90/AOC-PC

$\mathrm{AlT}$

A6T

Command 100D 


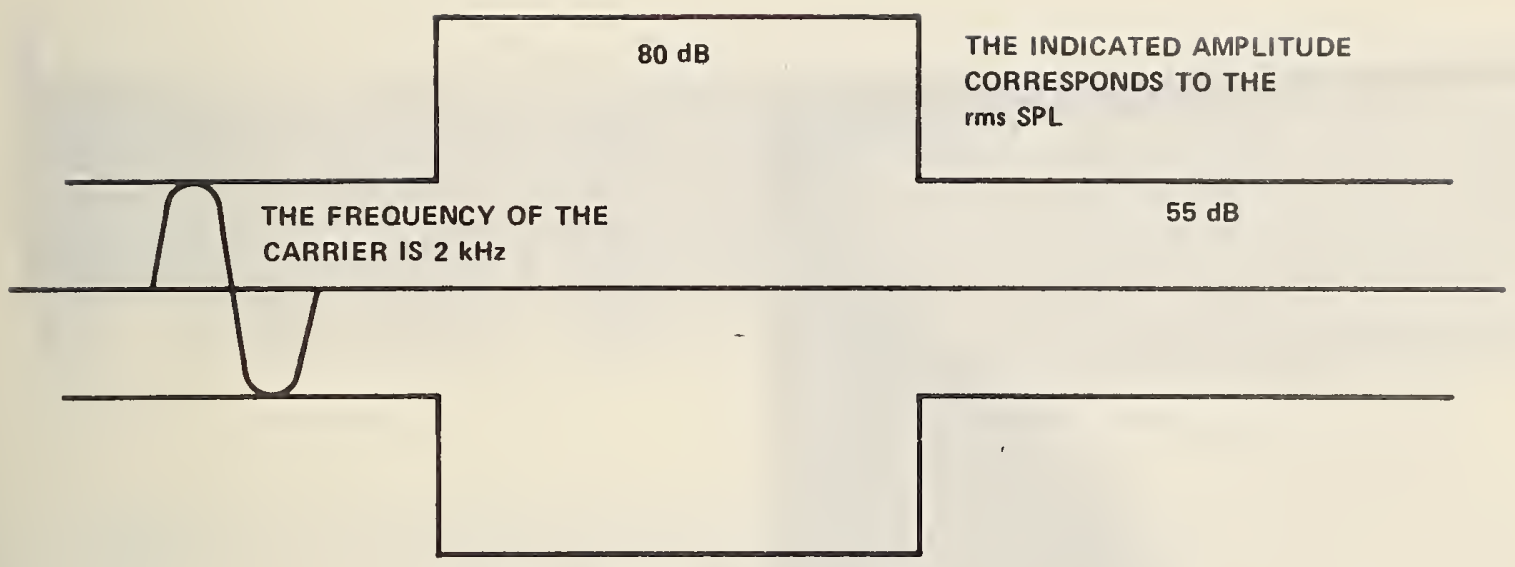

ENVELOPE OF INPUT SIGNAL
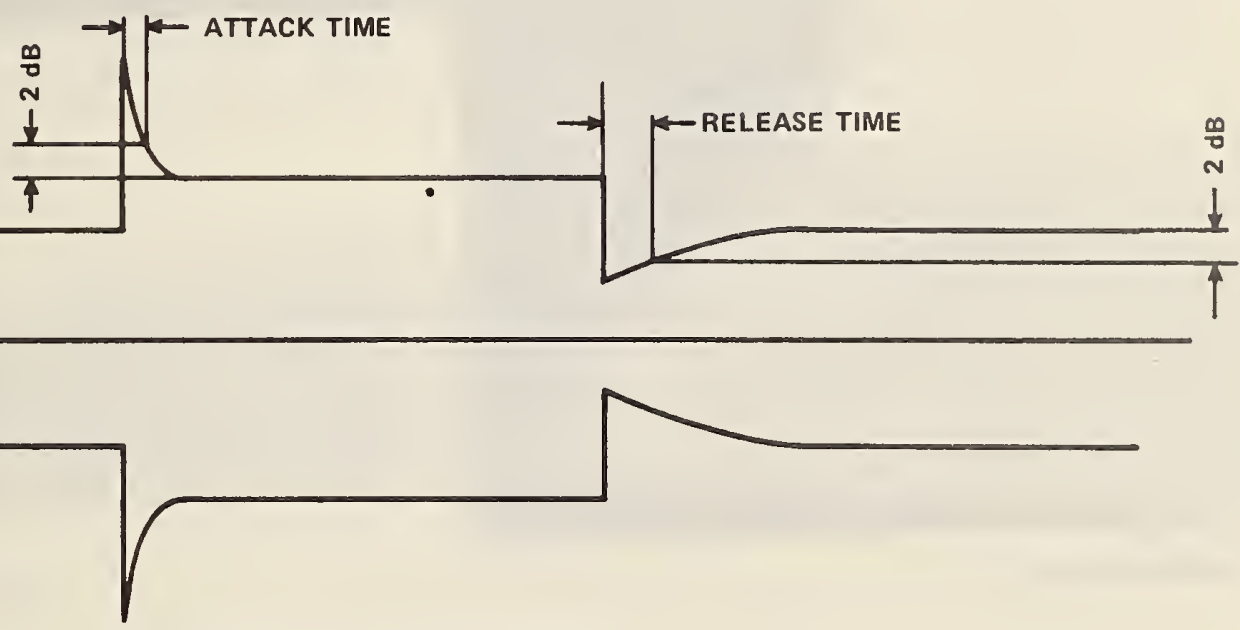

ENVELOPE OF OUTPUT SIGNAL

Fig. 1. Idealized envelopes of hearing aid input and output signals. 


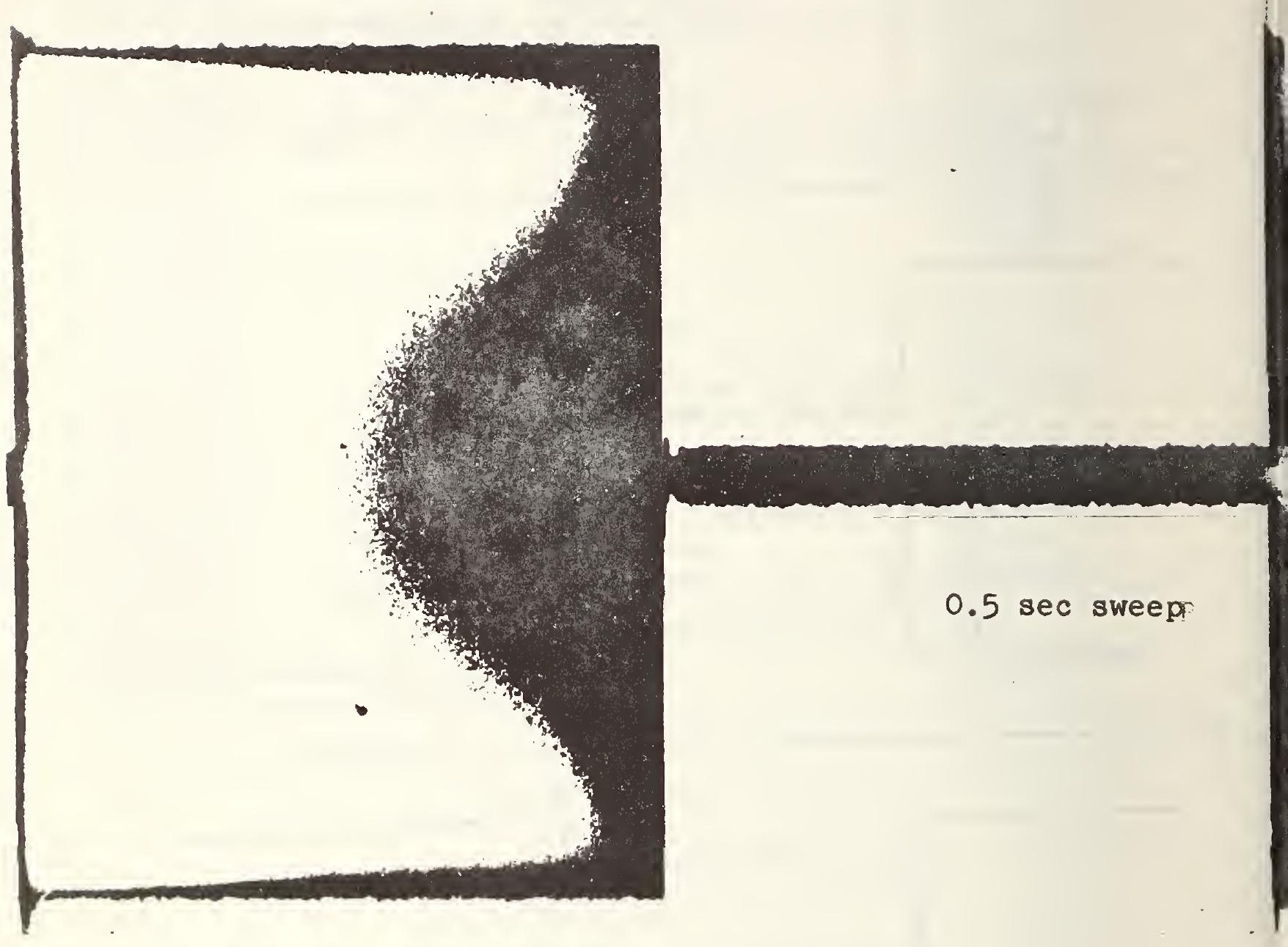

Fig 2 Voltage output from the reference microphone. 

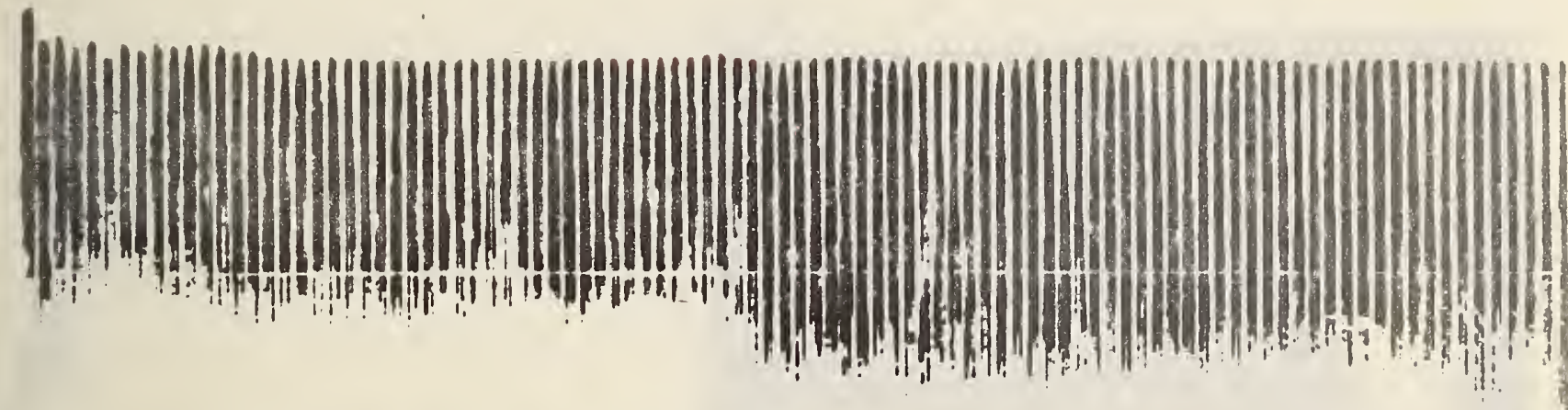

\section{$0.05 \mathrm{sec}$ sweep}

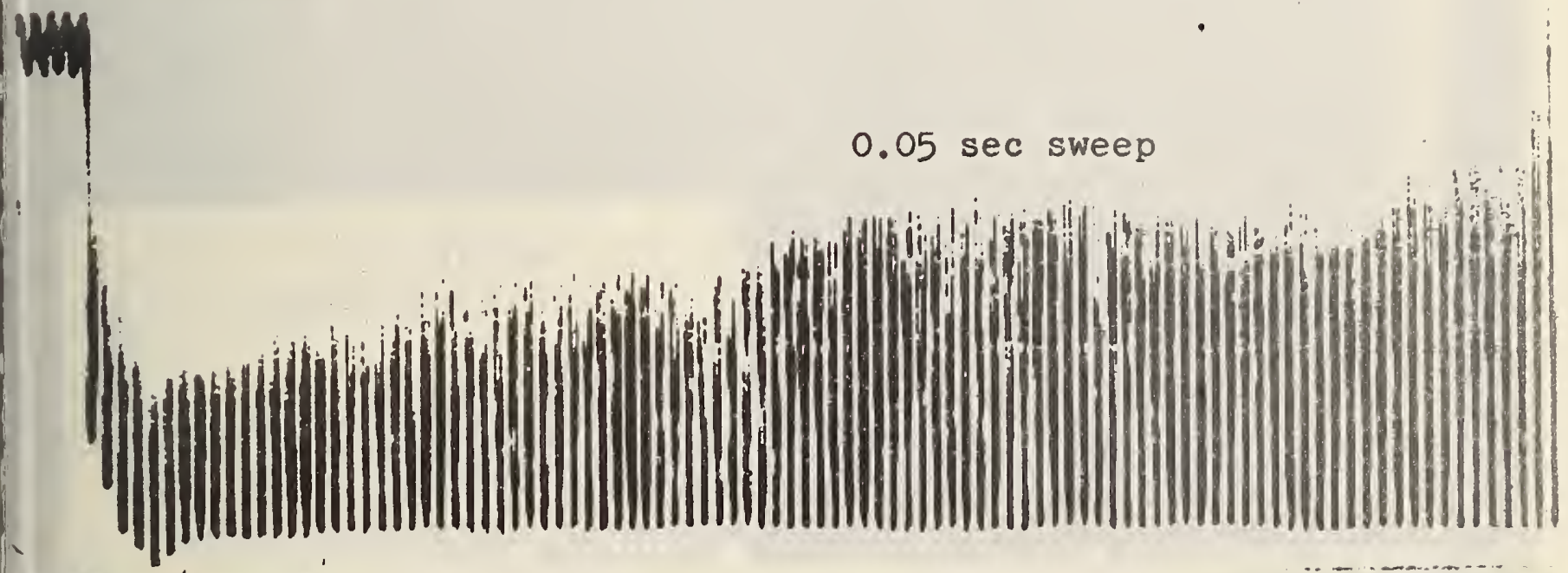

Fig. 3 Voltage output from the reference microphone viewed with an expanded time scale. 


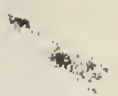

\section{5}

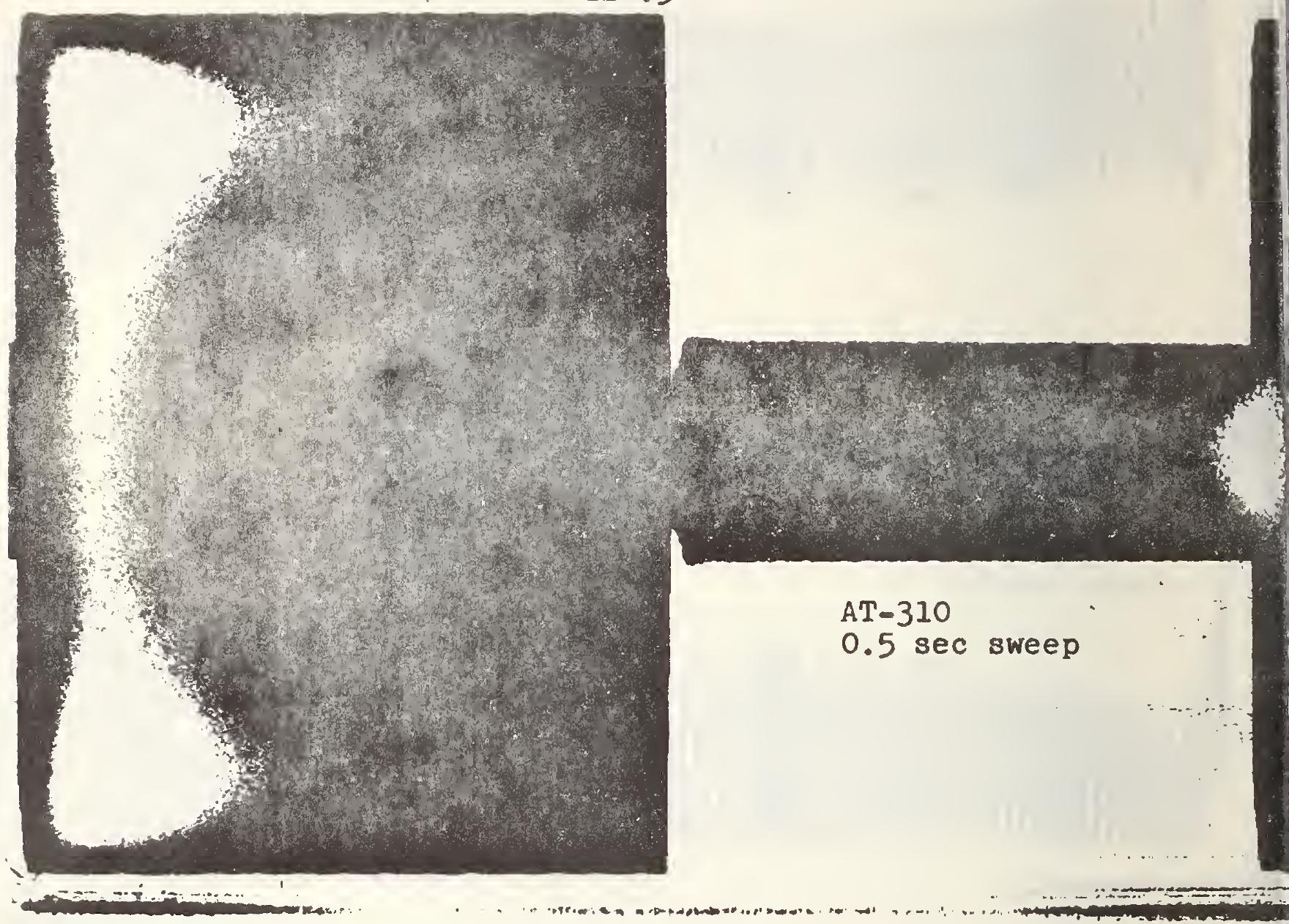




\section{5}

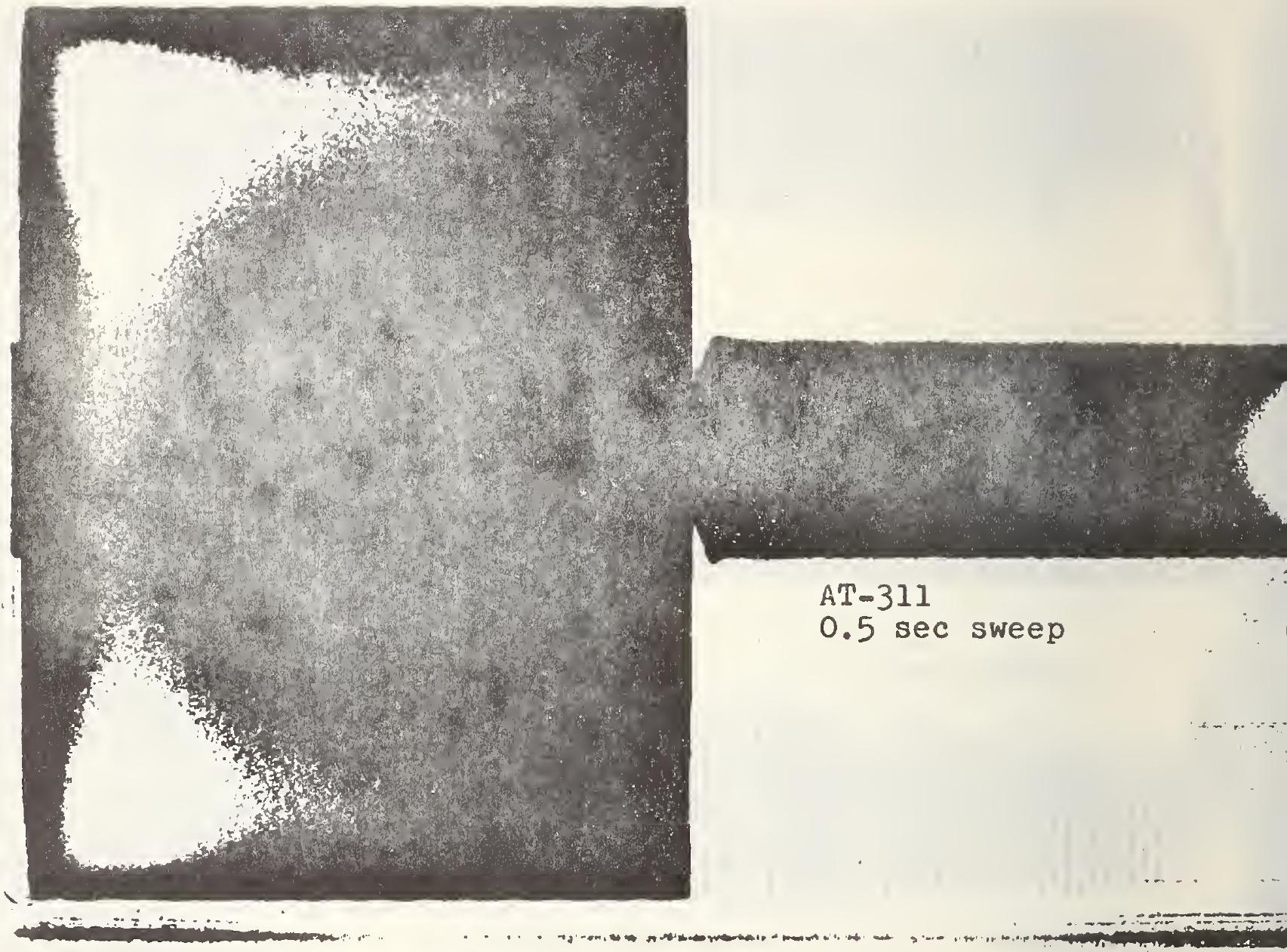




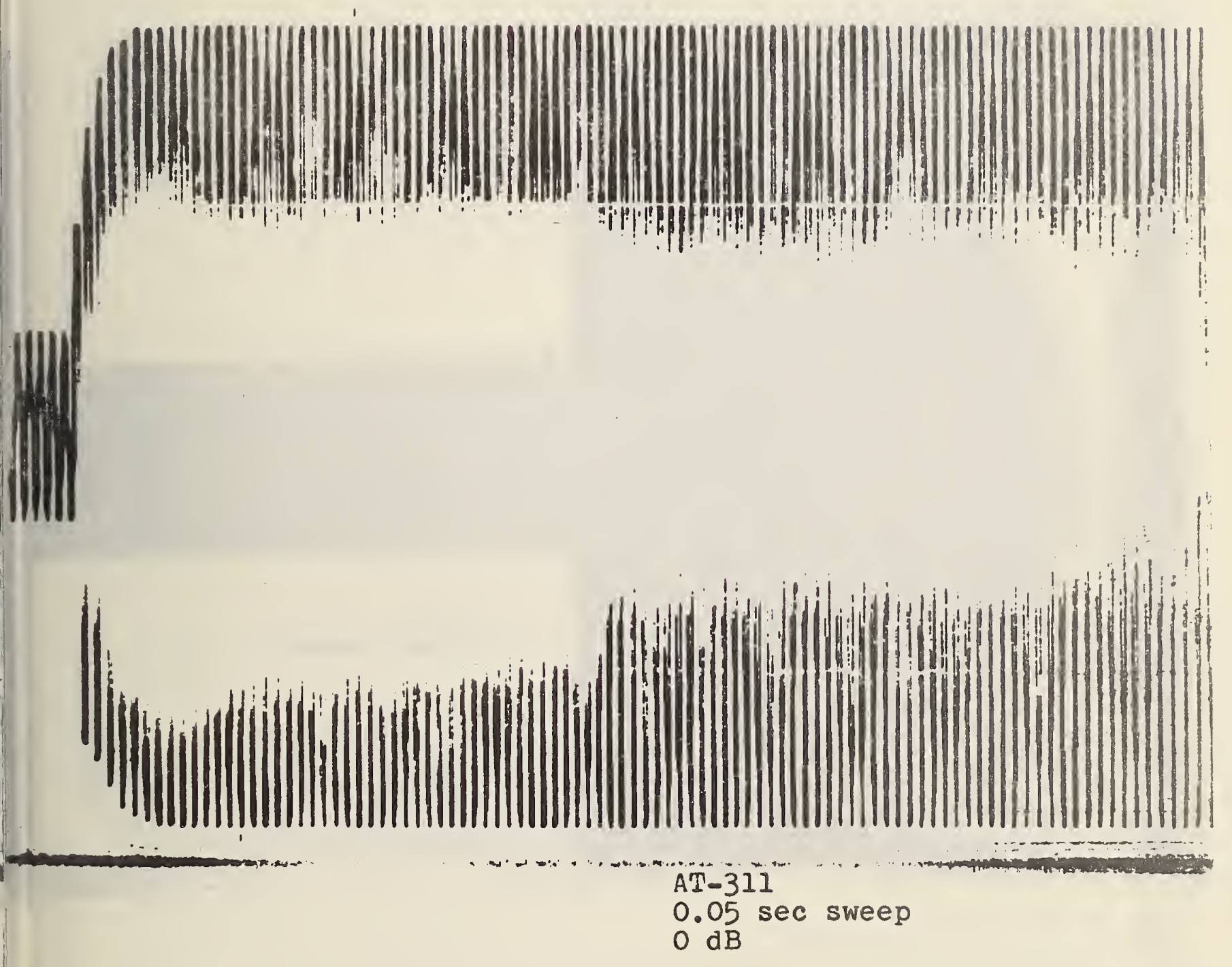


117

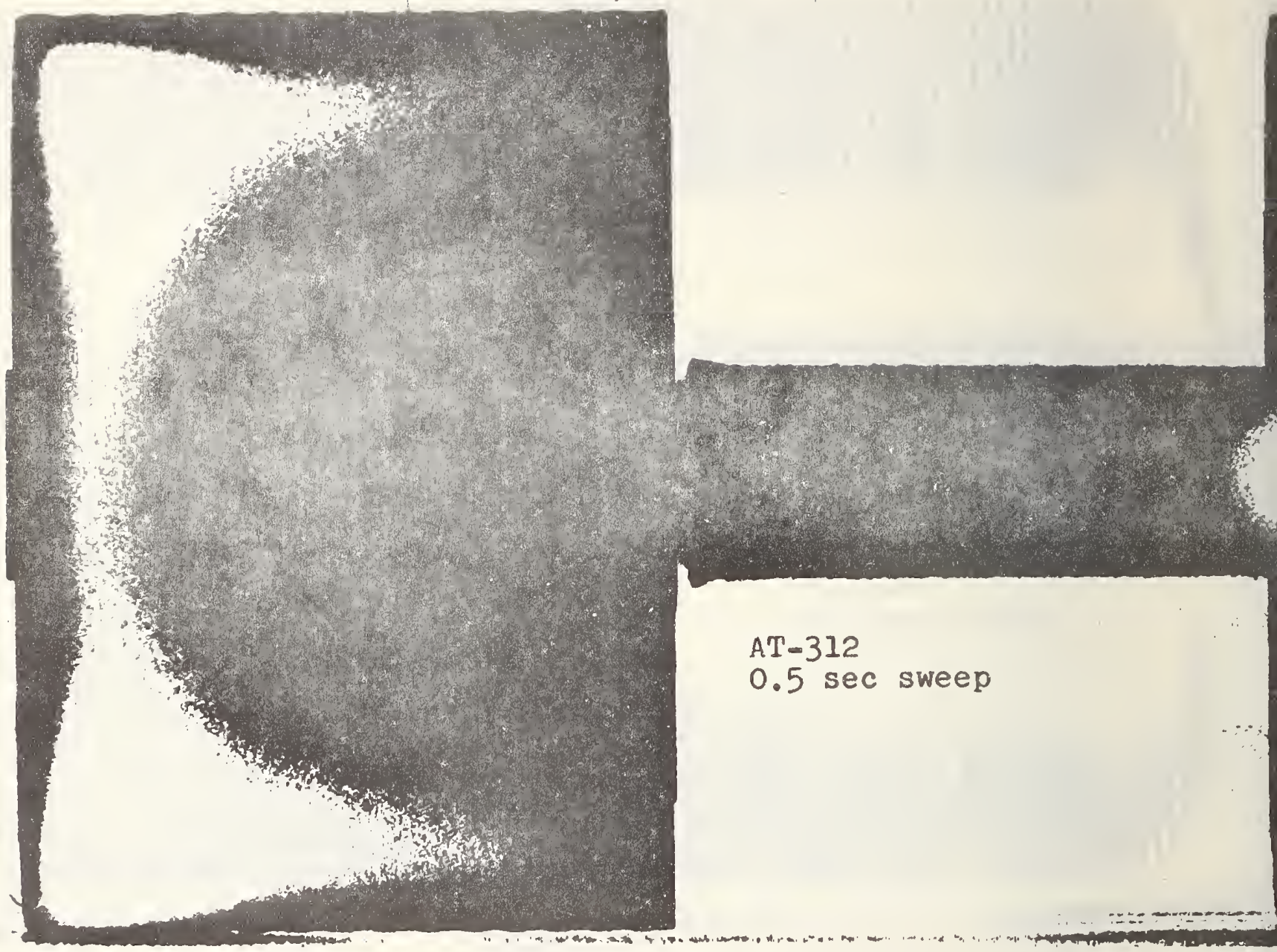




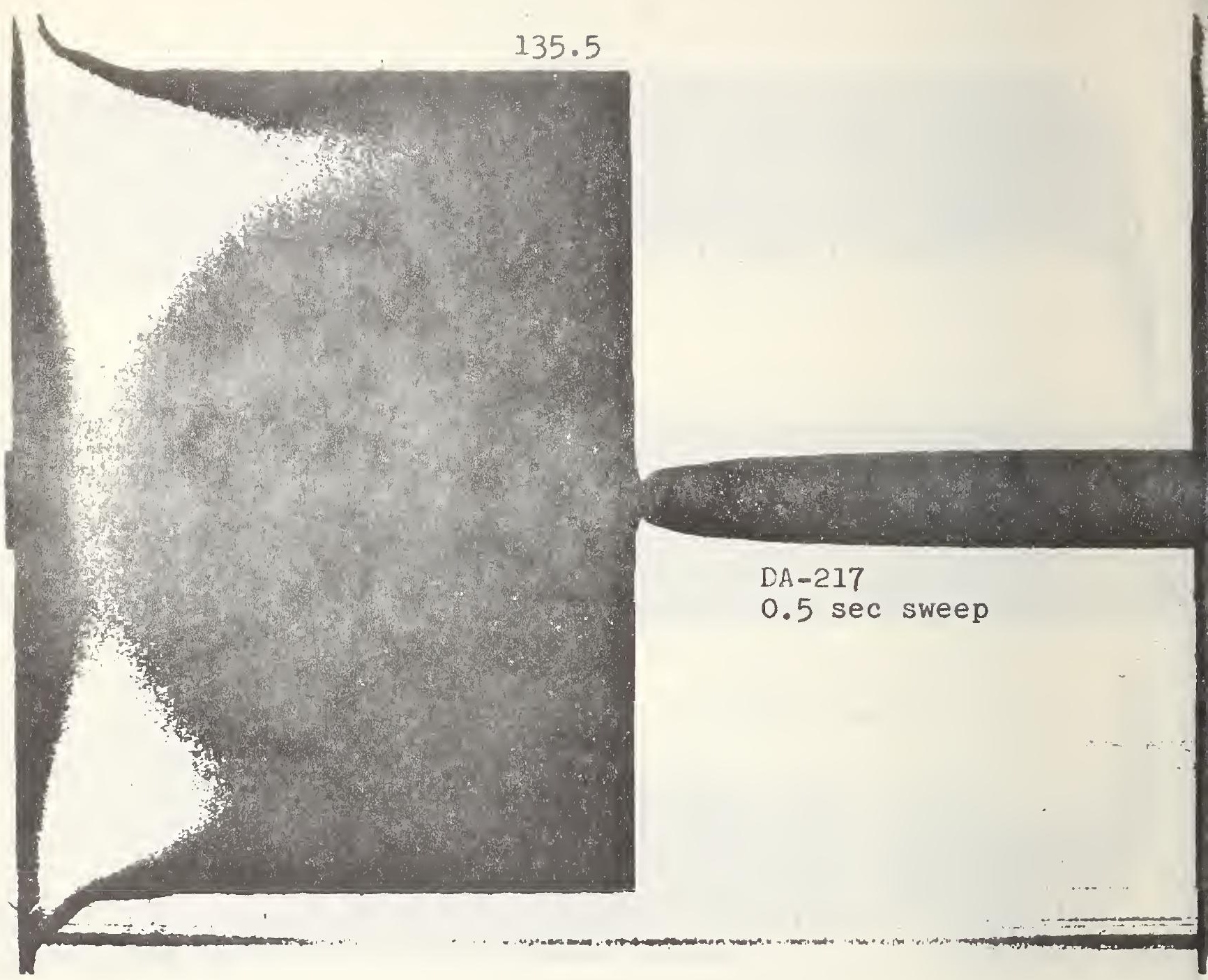




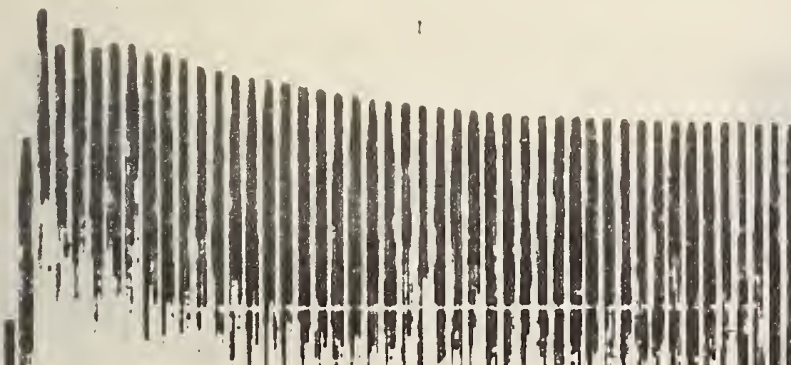

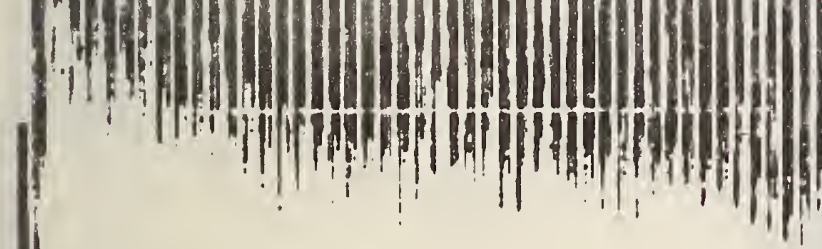
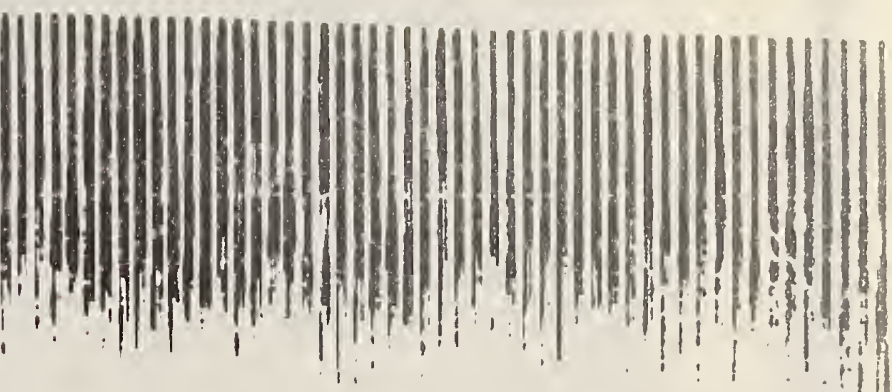

$$
\begin{aligned}
& D A-217 \\
& 0.05 \text { sec sweep } \\
& -2 \mathrm{~dB}
\end{aligned}
$$




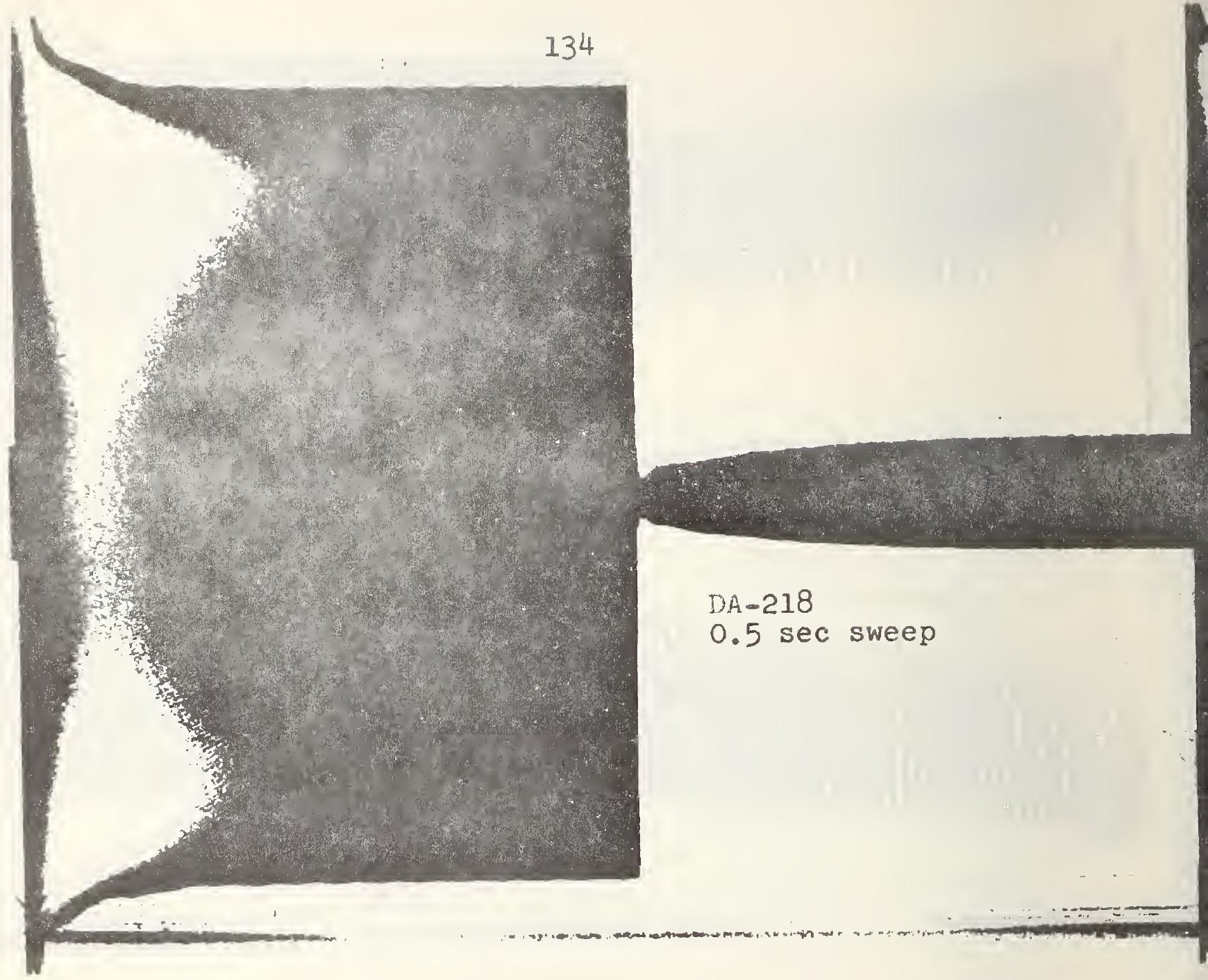




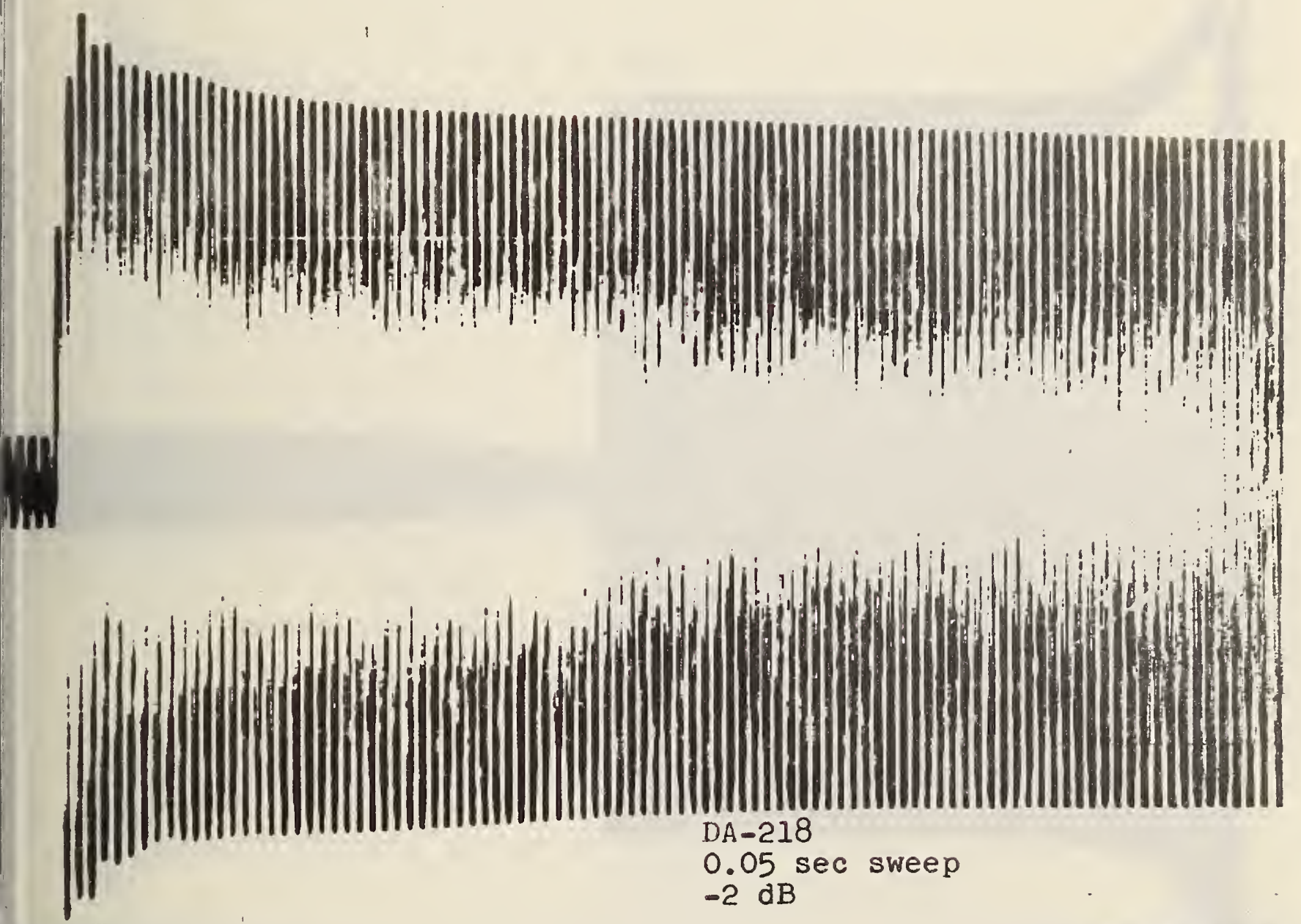




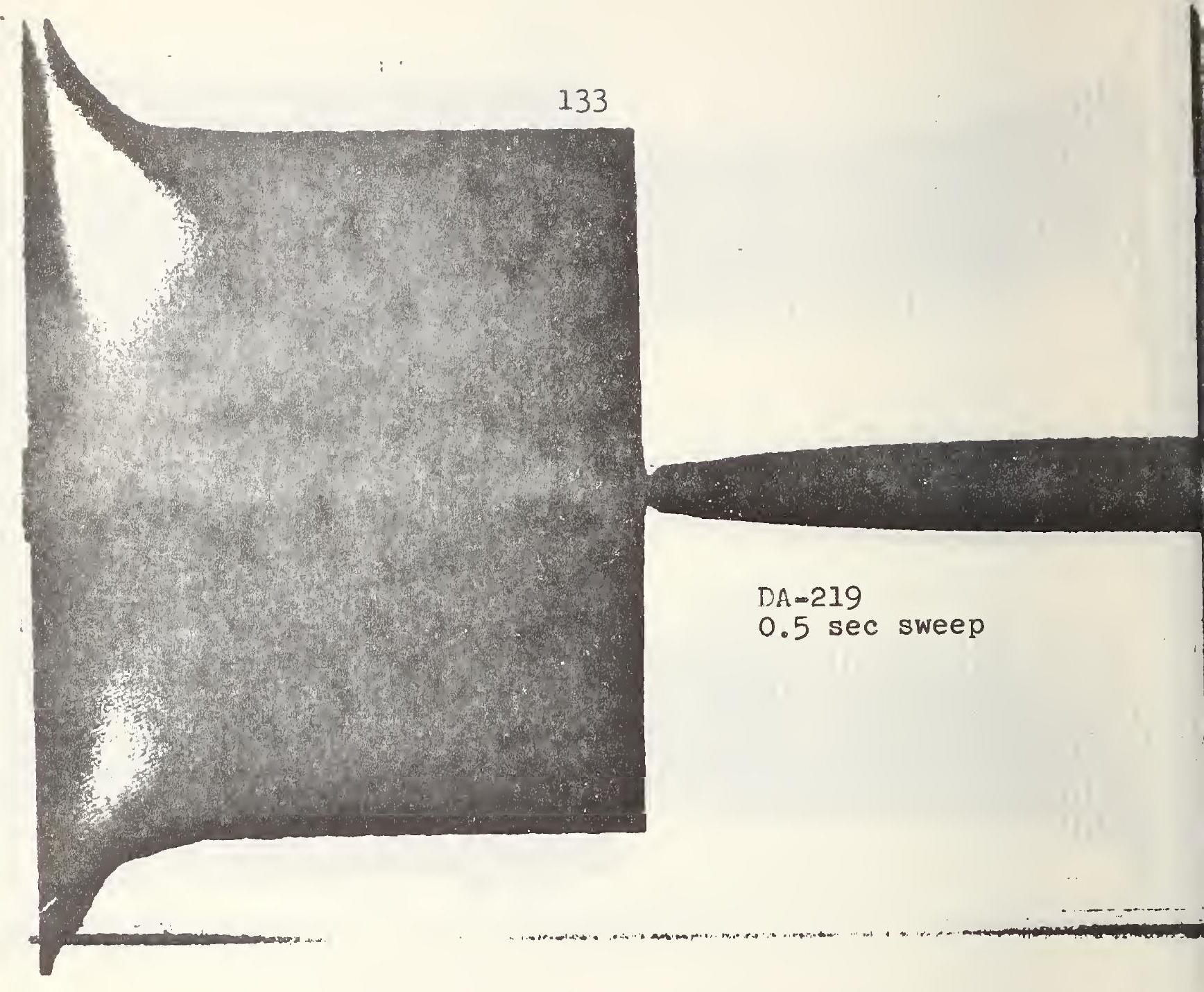




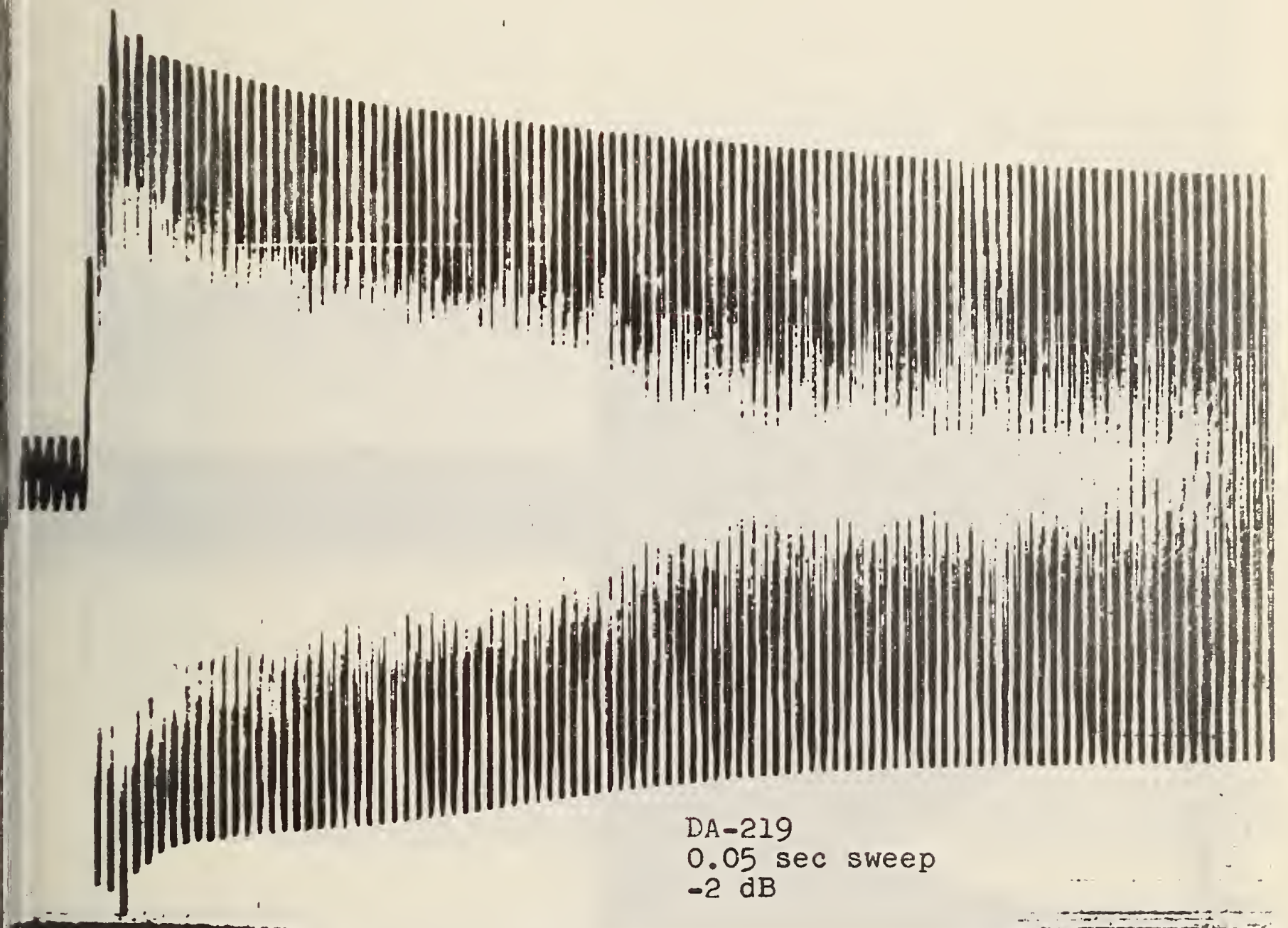




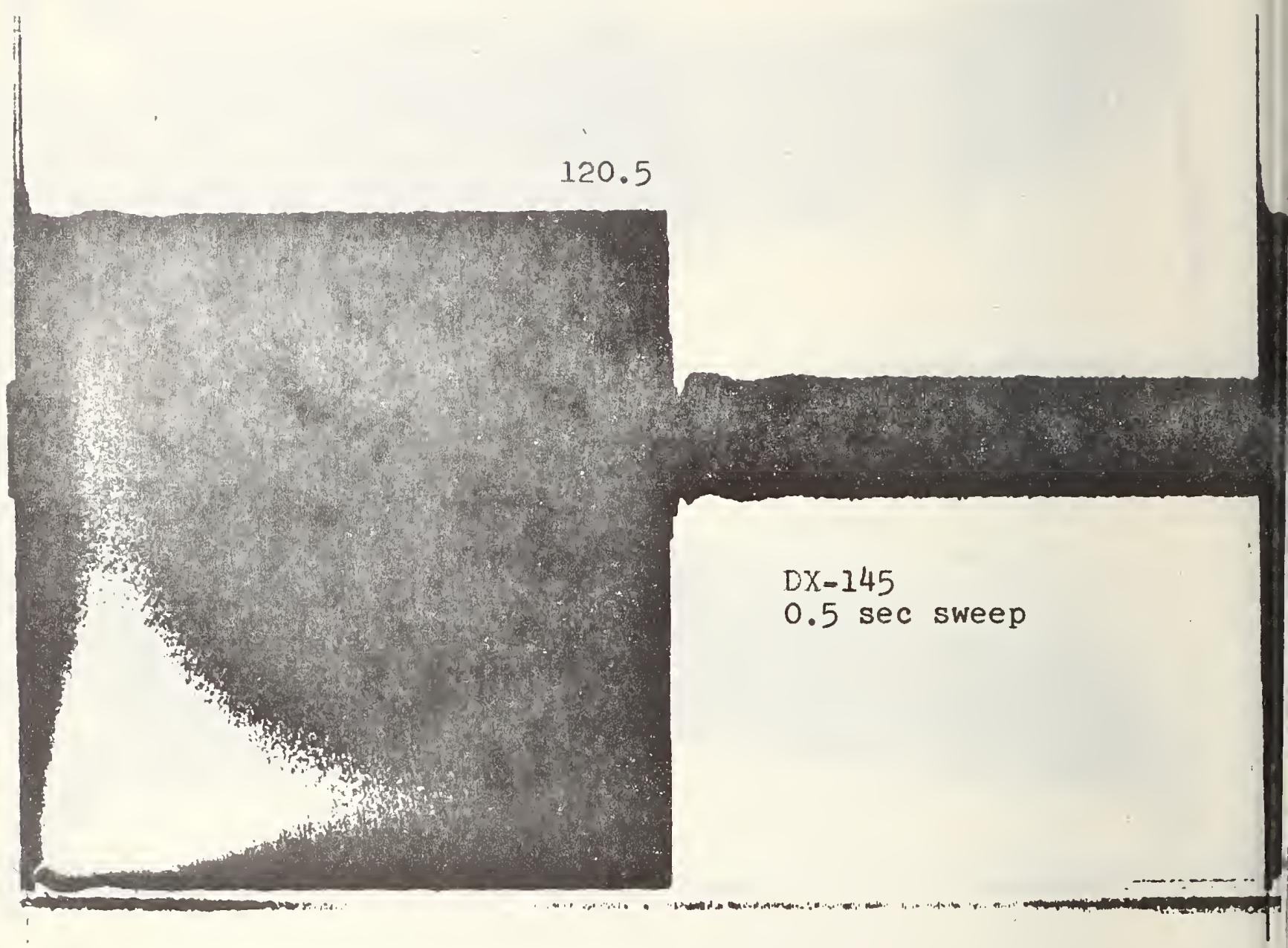


, A

$$
\begin{aligned}
& D X-145 \\
& 0.05 \text { sec sweep } \\
& -1 \mathrm{~dB}
\end{aligned}
$$

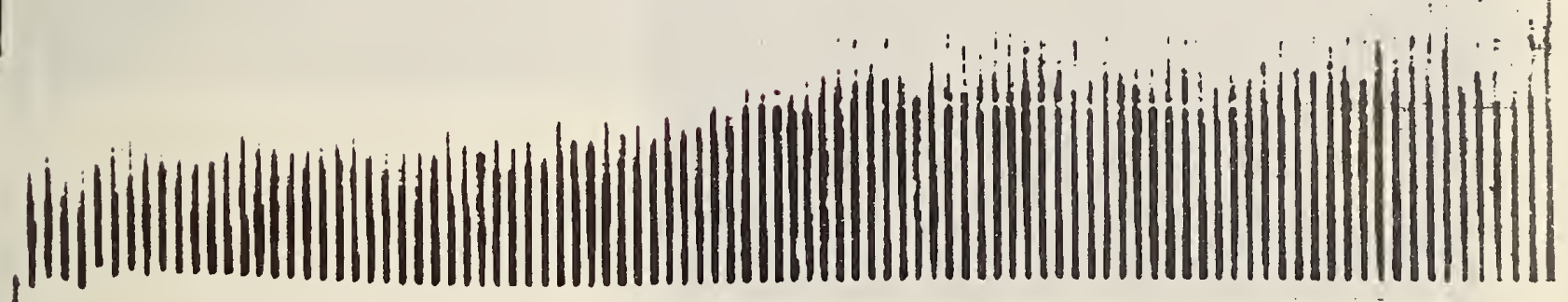




\section{5}

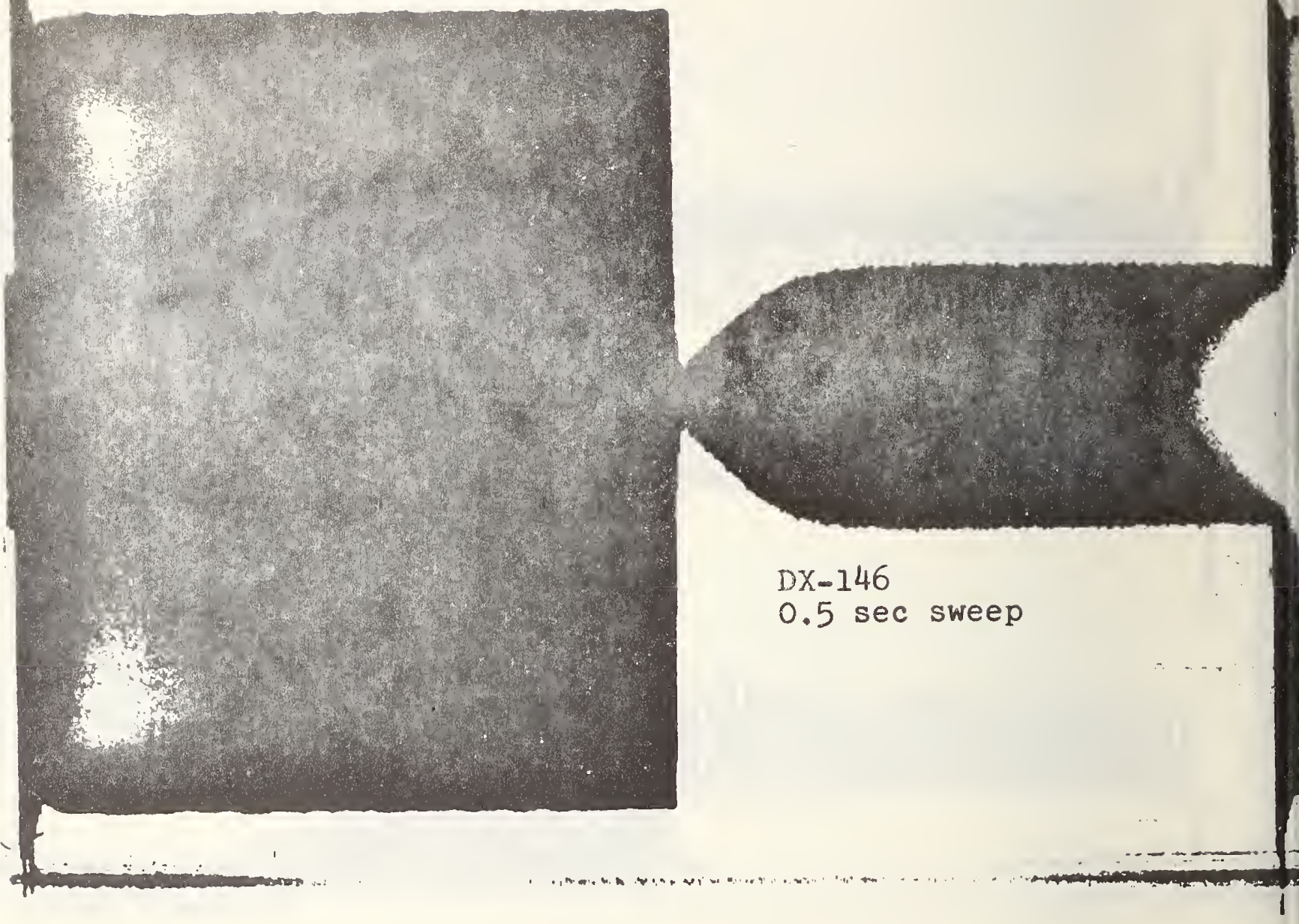




\section{5}

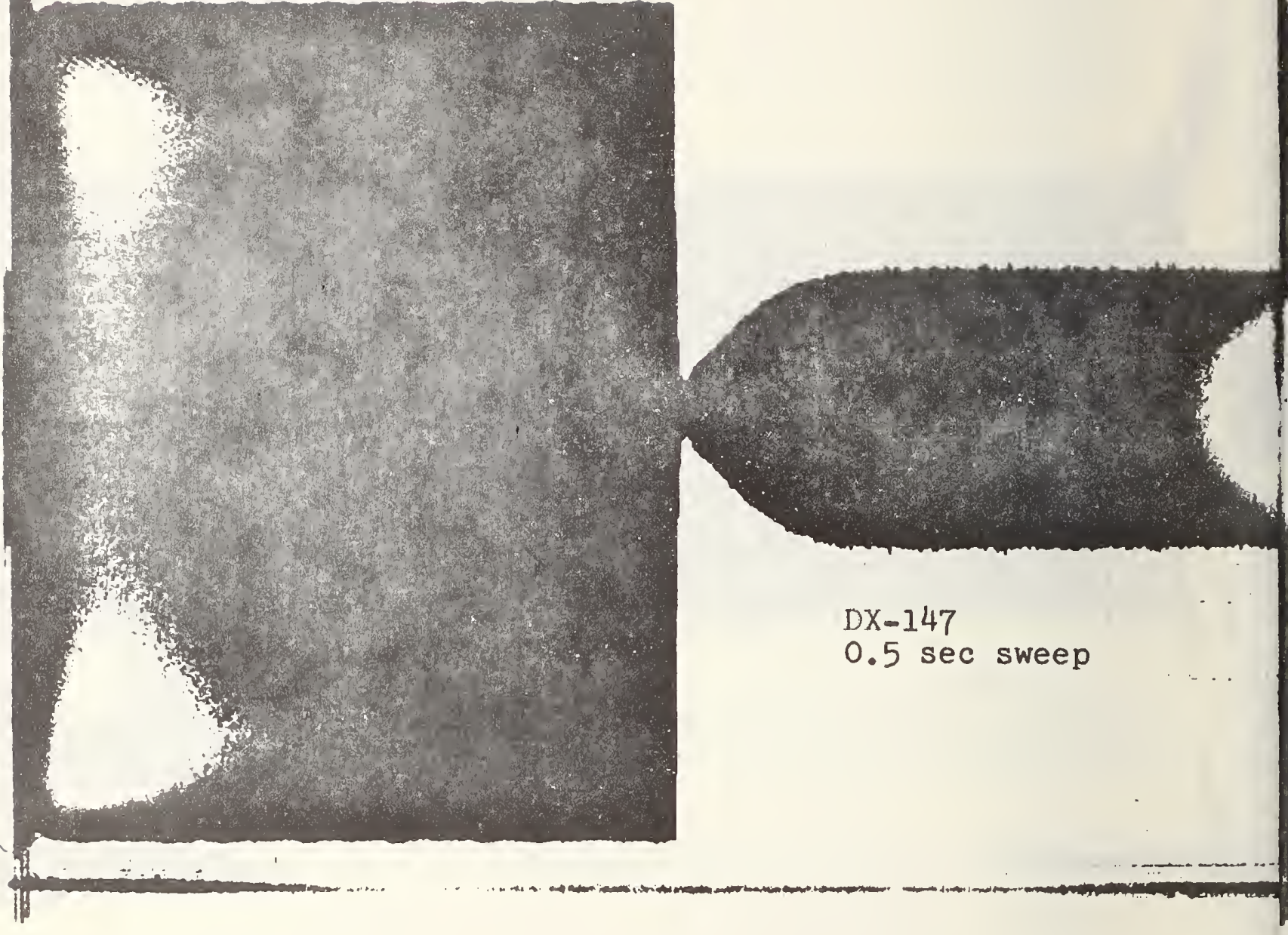




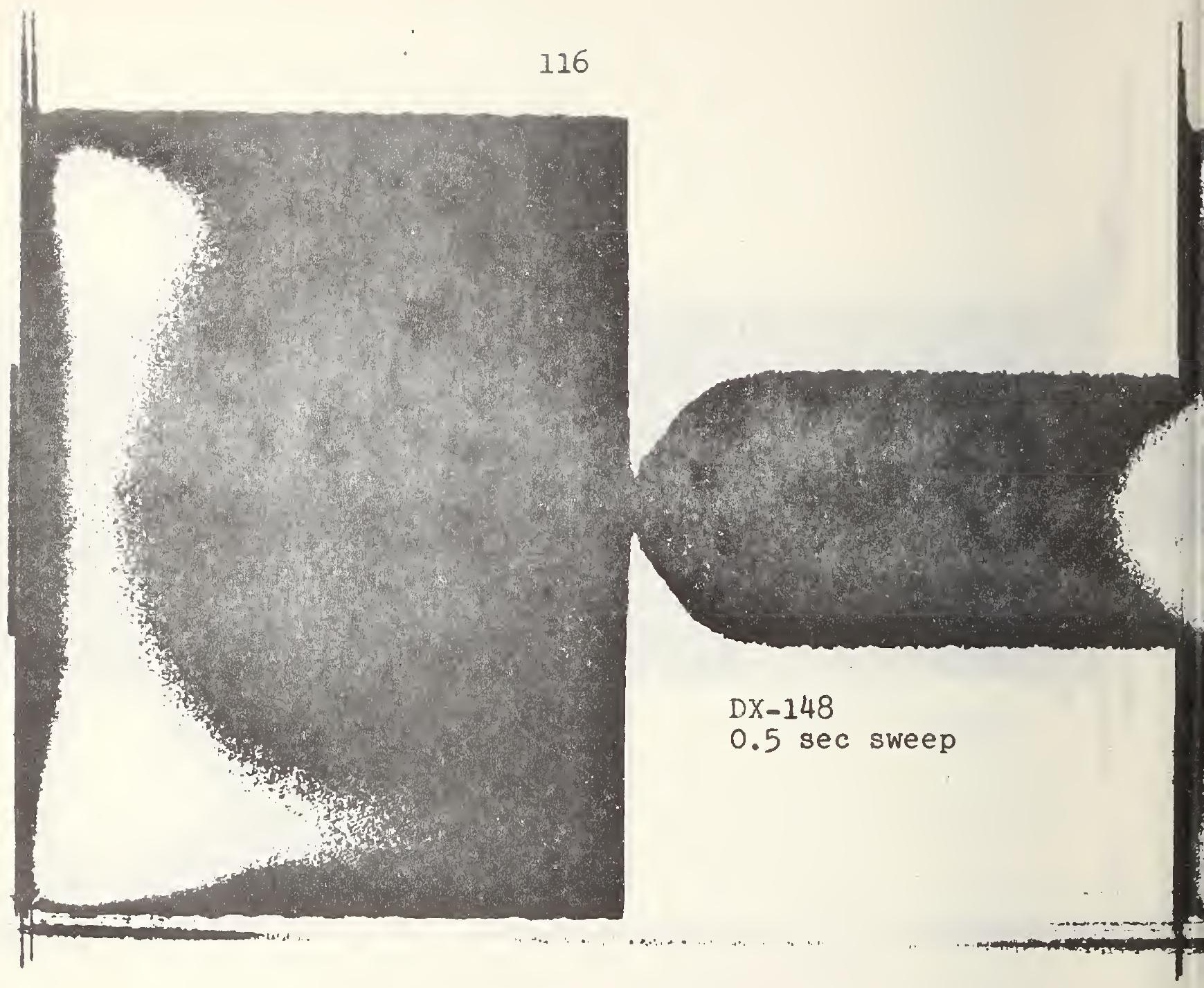




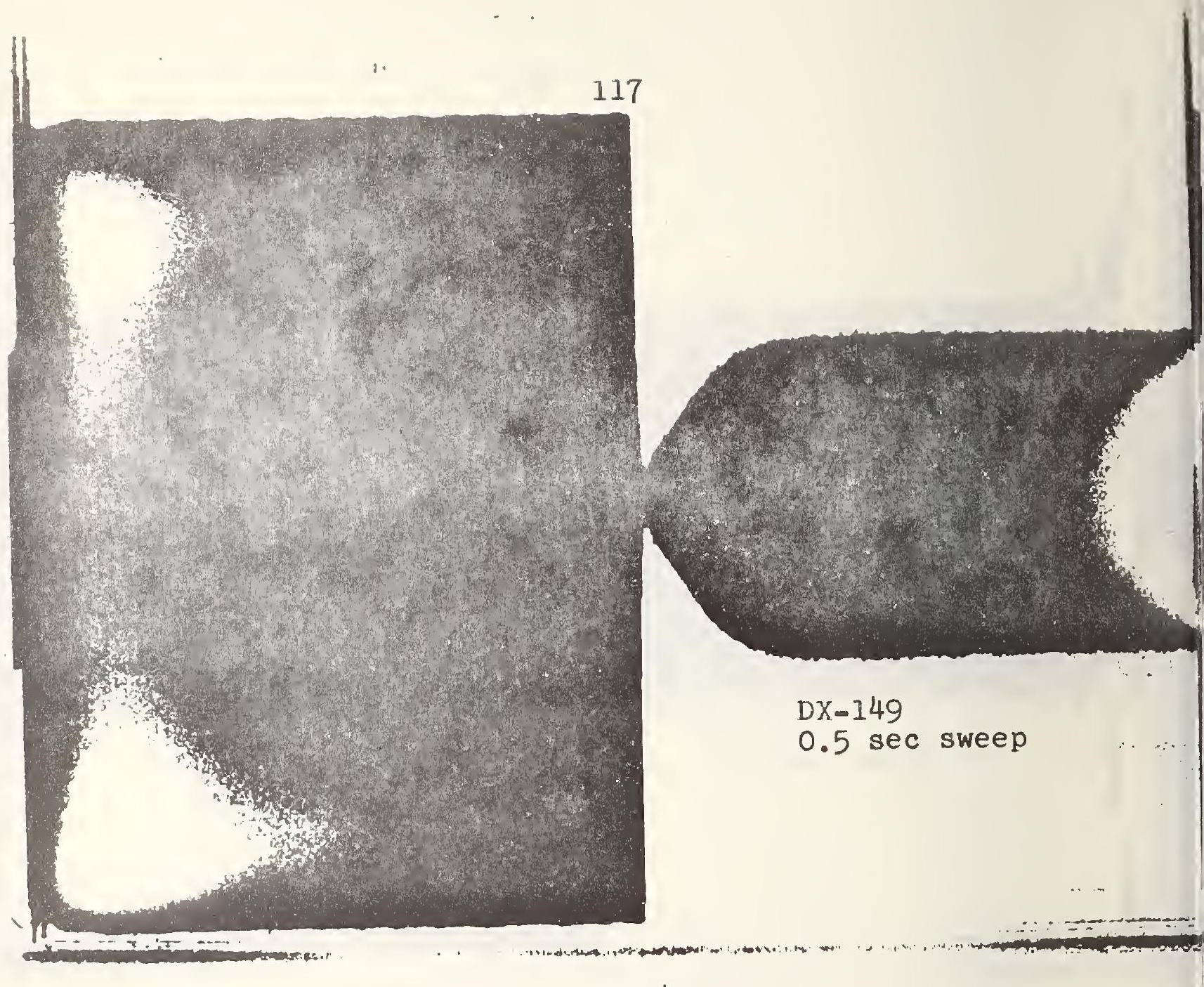




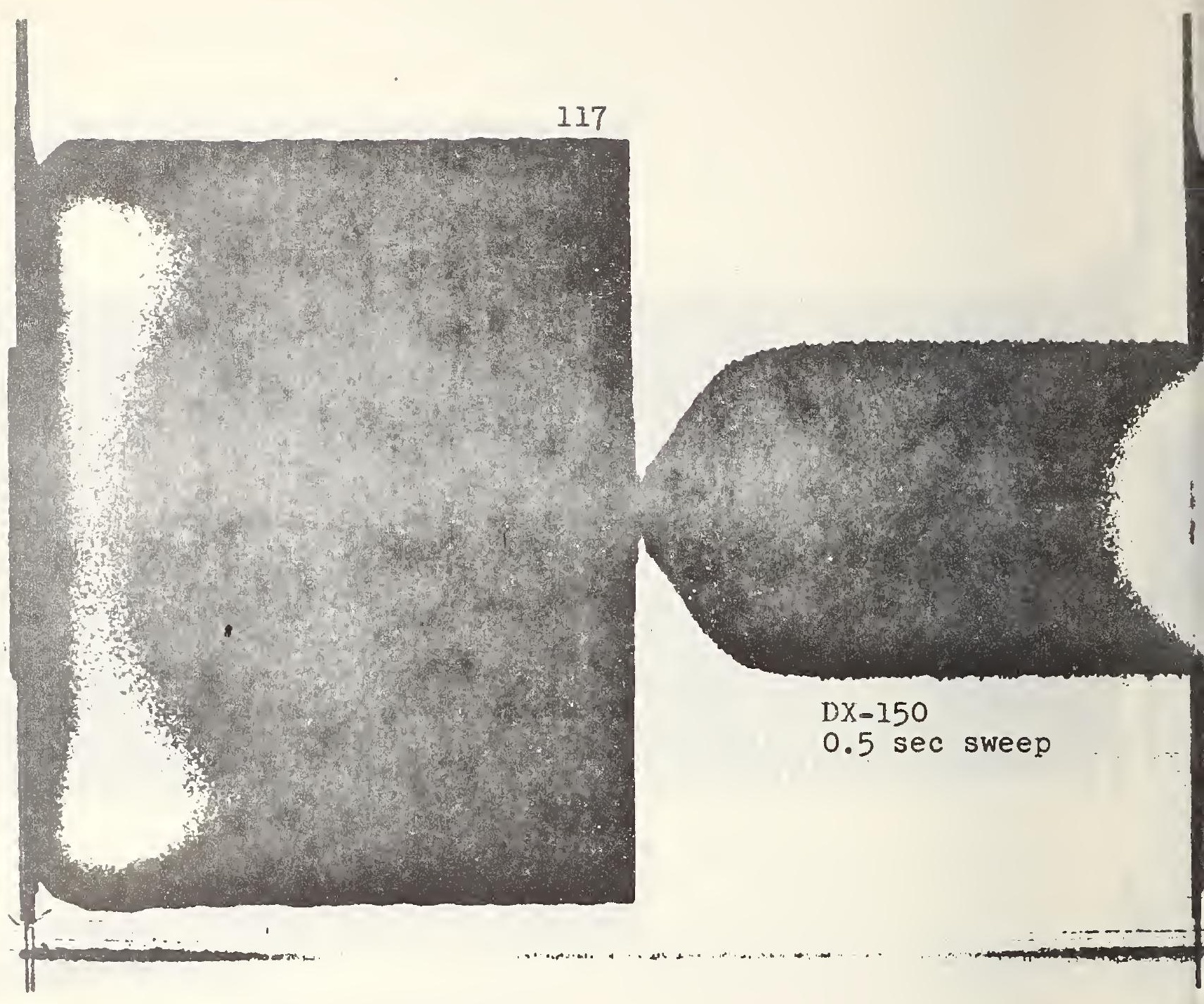




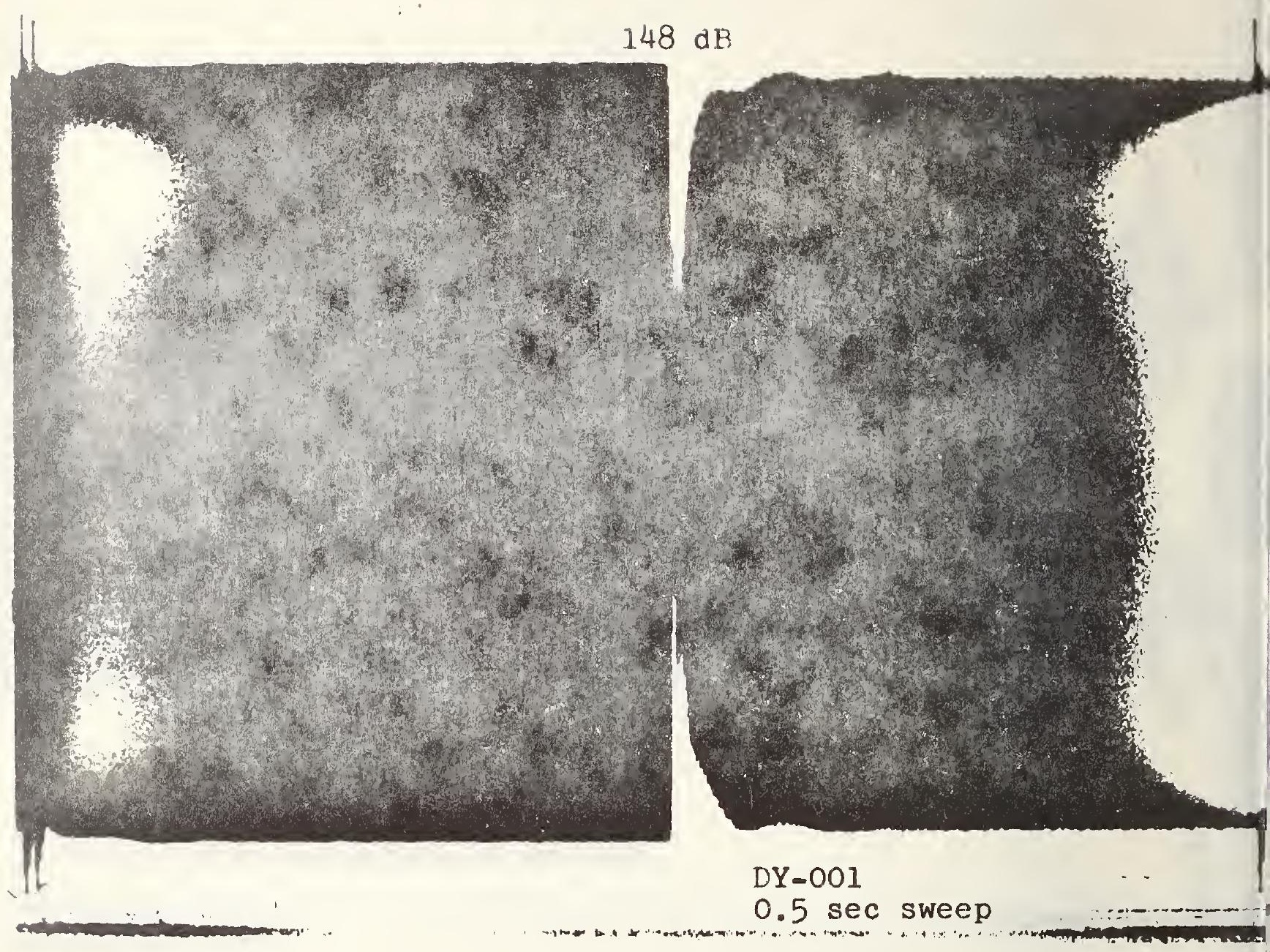




\section{7}
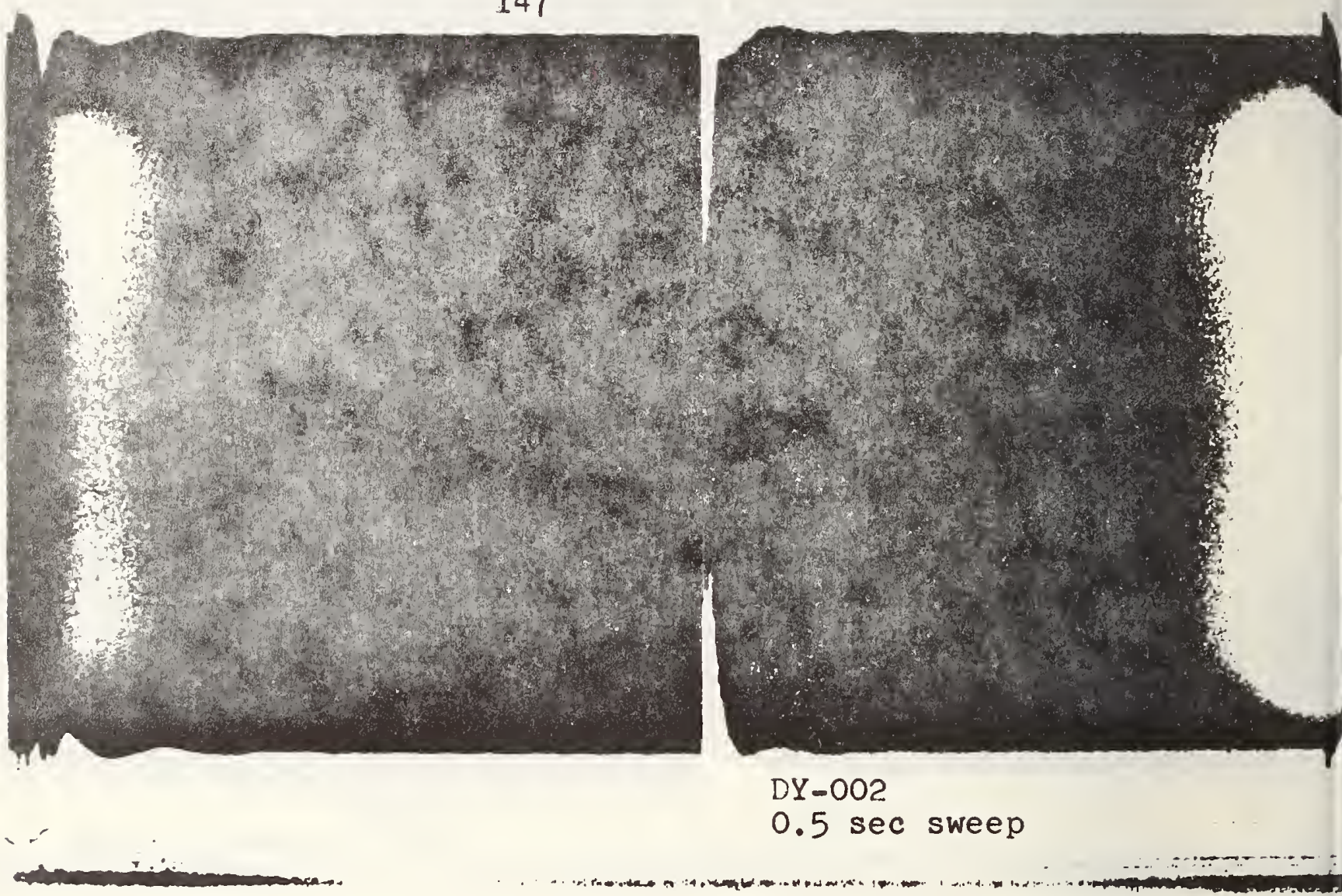


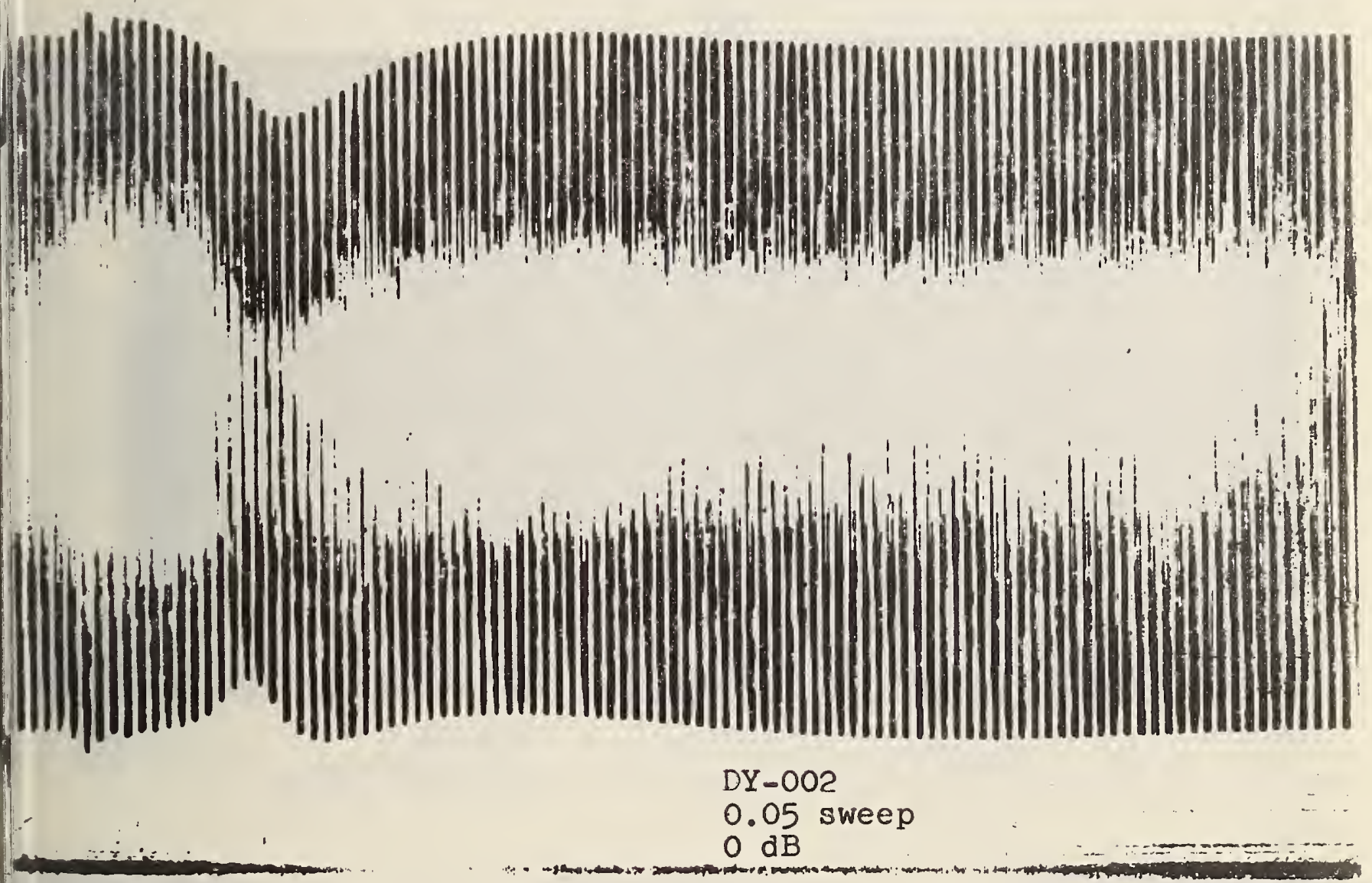




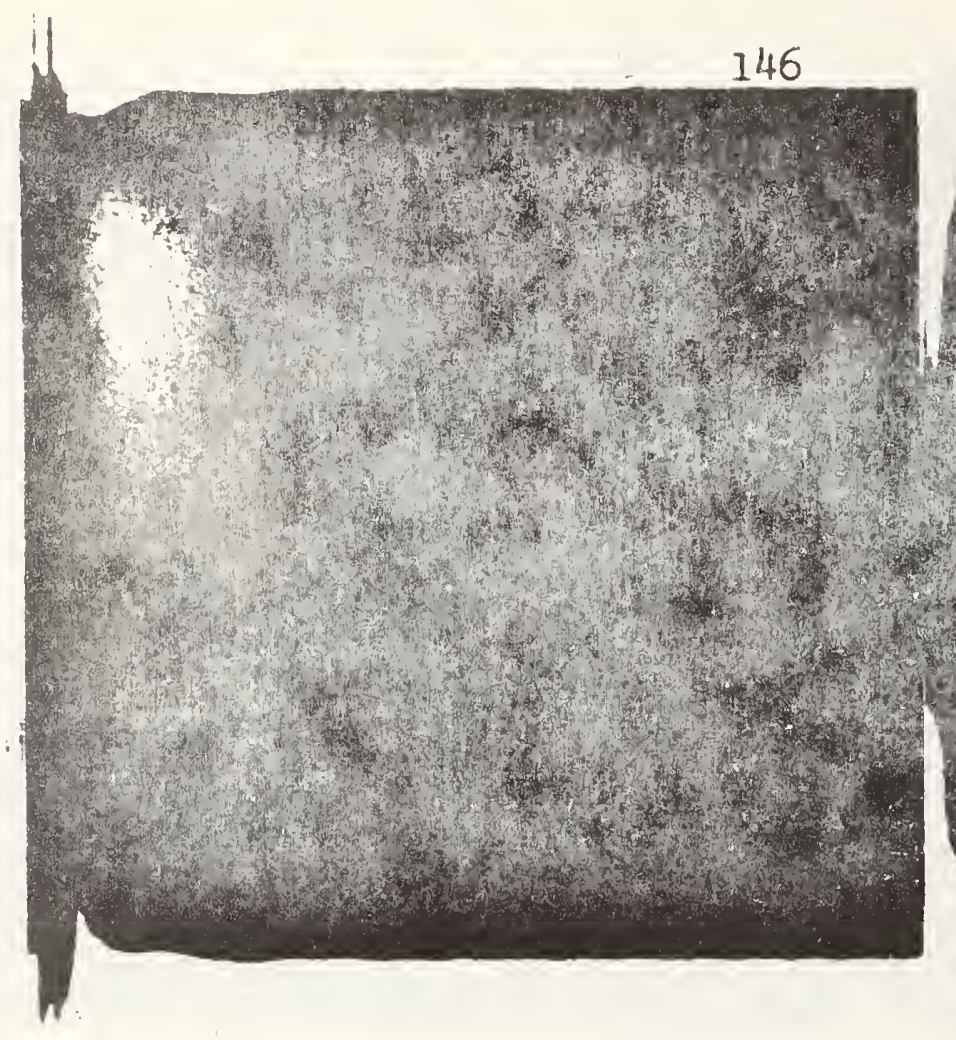

146
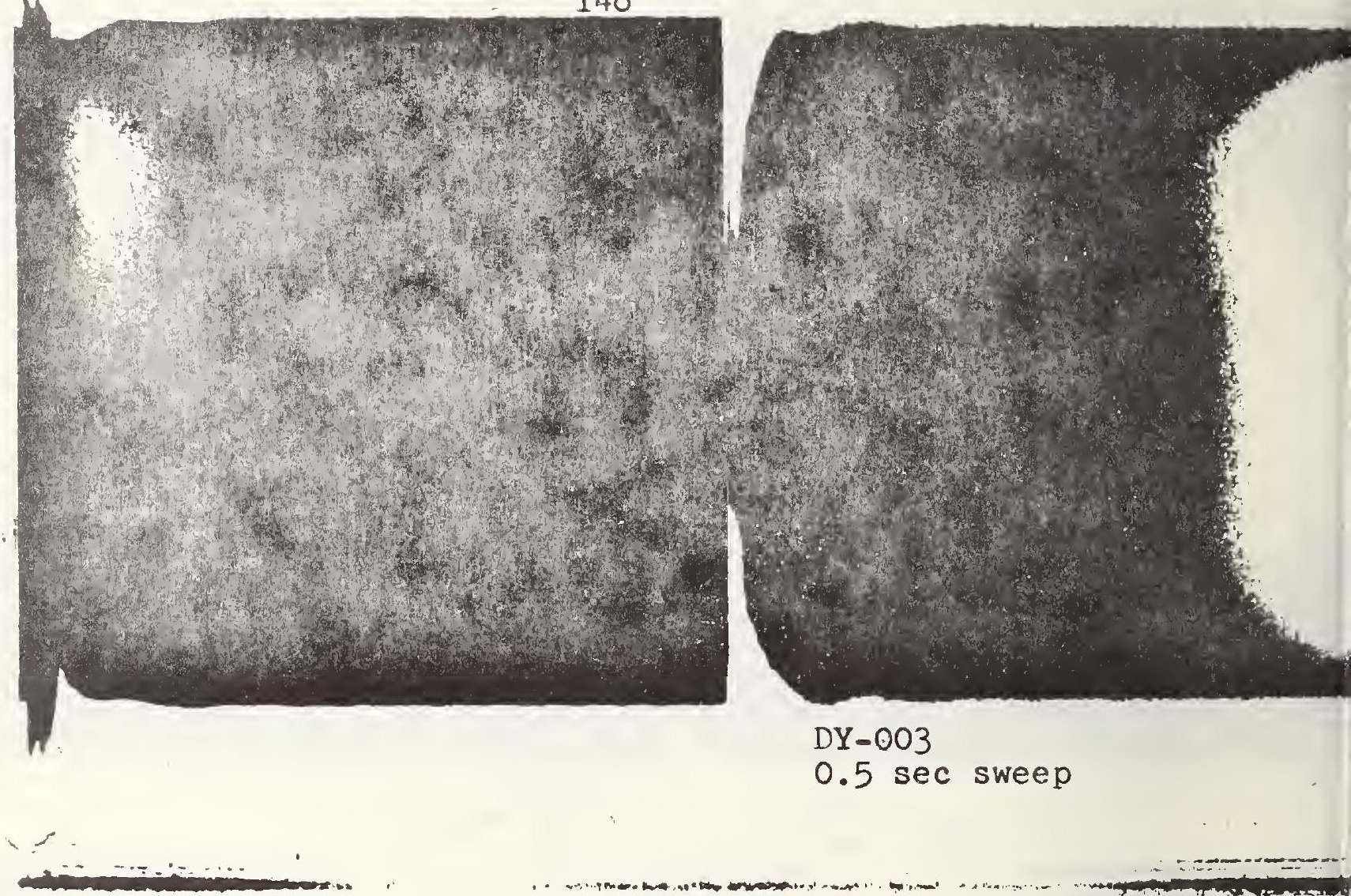


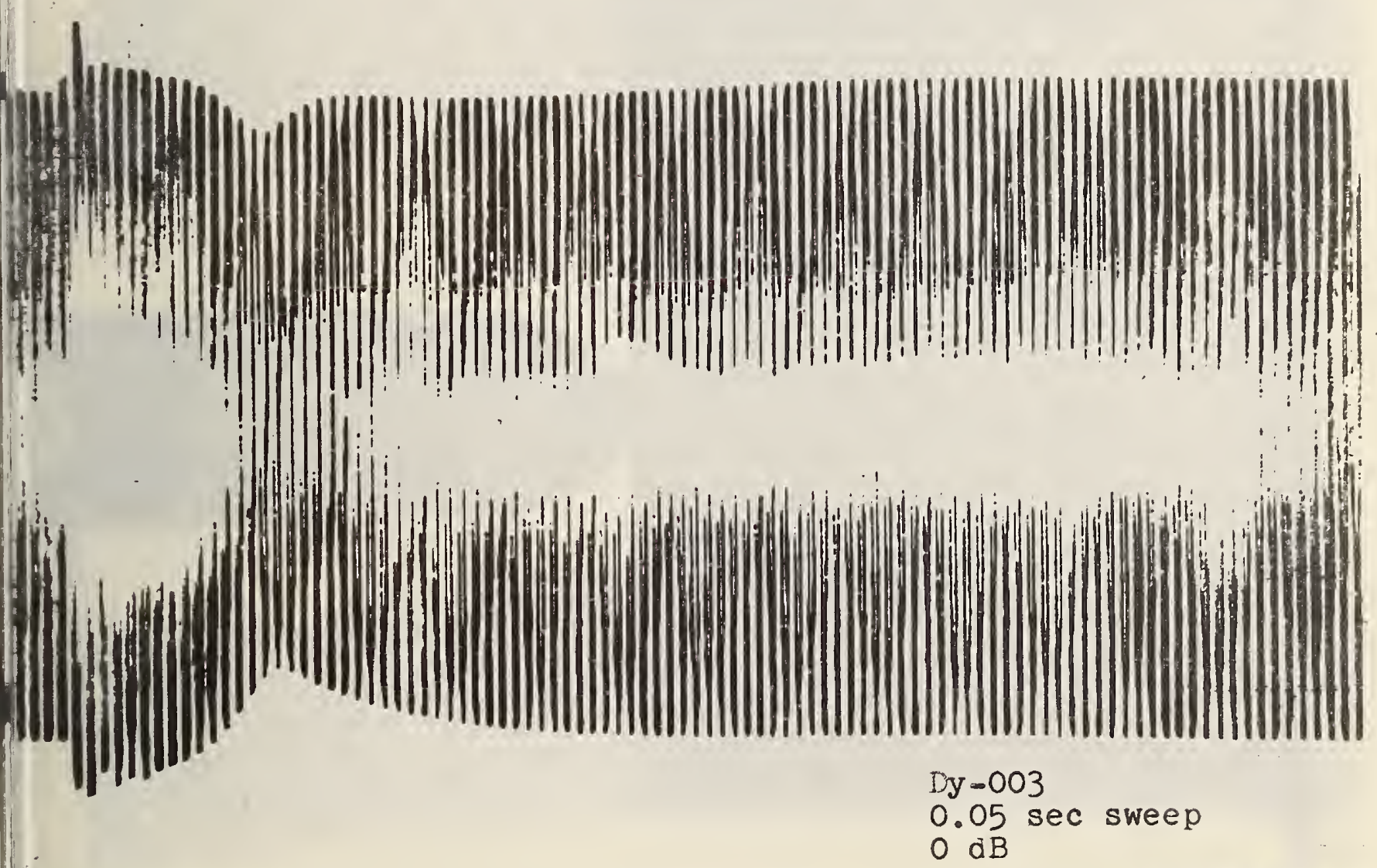




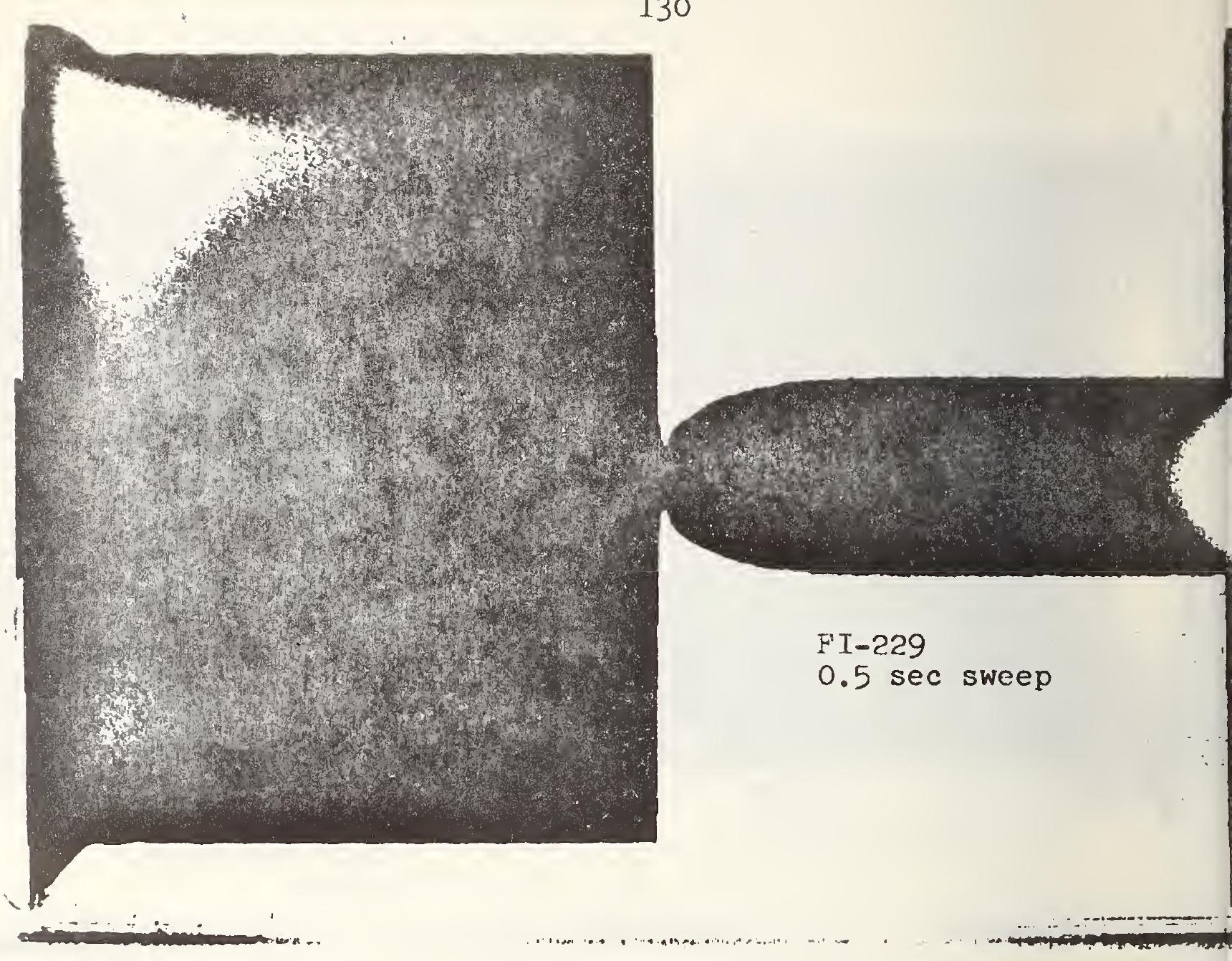




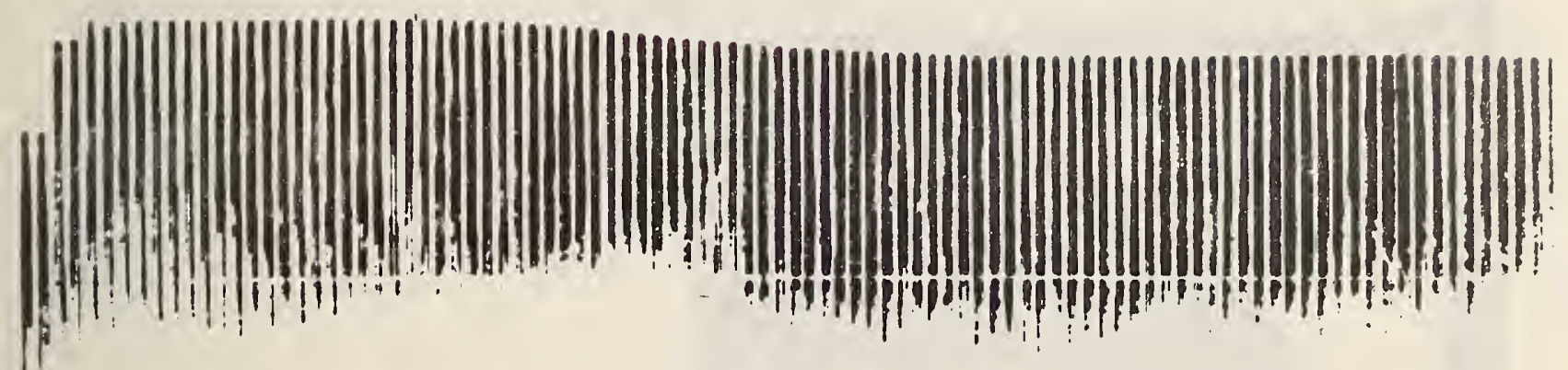

$$
\begin{aligned}
& \text { FI }-229 \\
& 0.05 \text { sec sweep } \\
& 0 \text { dB }
\end{aligned}
$$
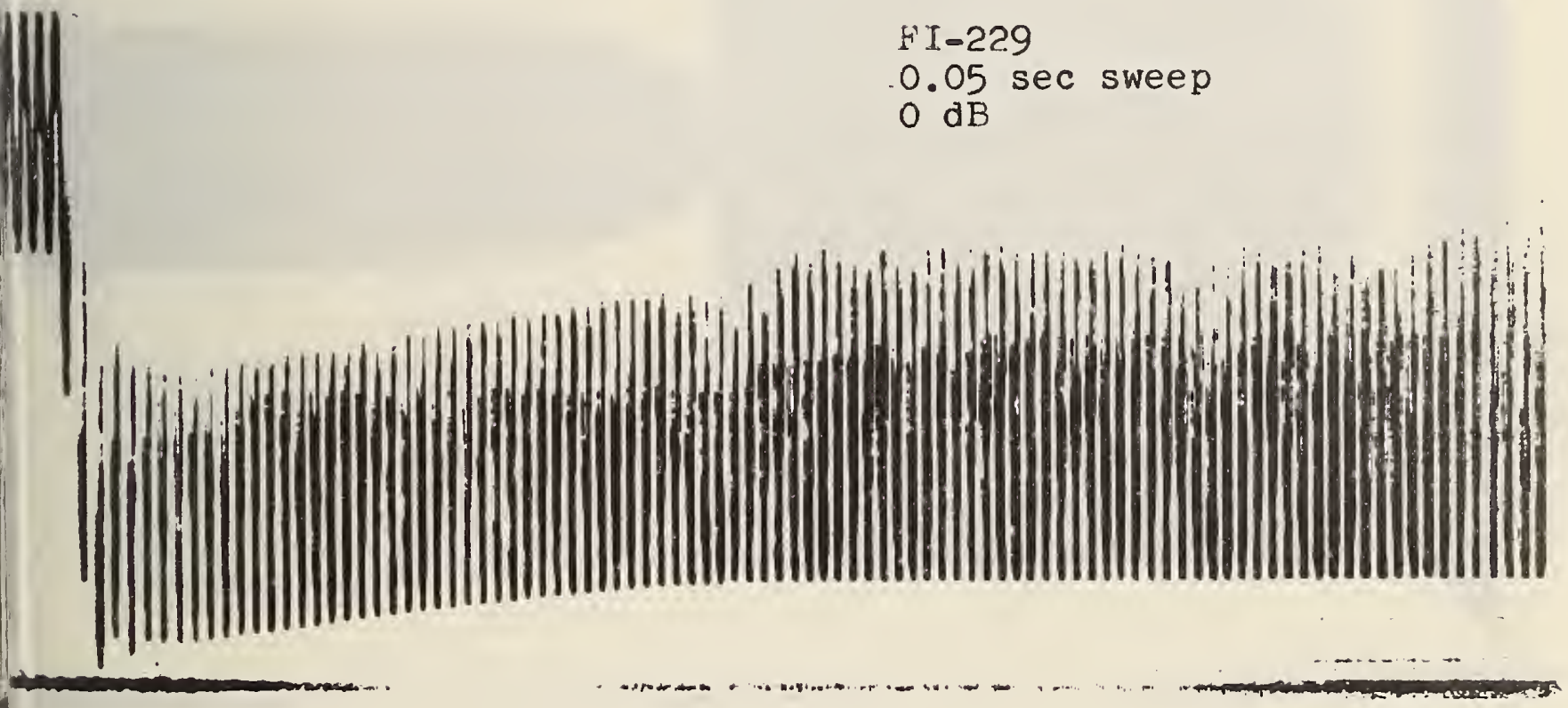


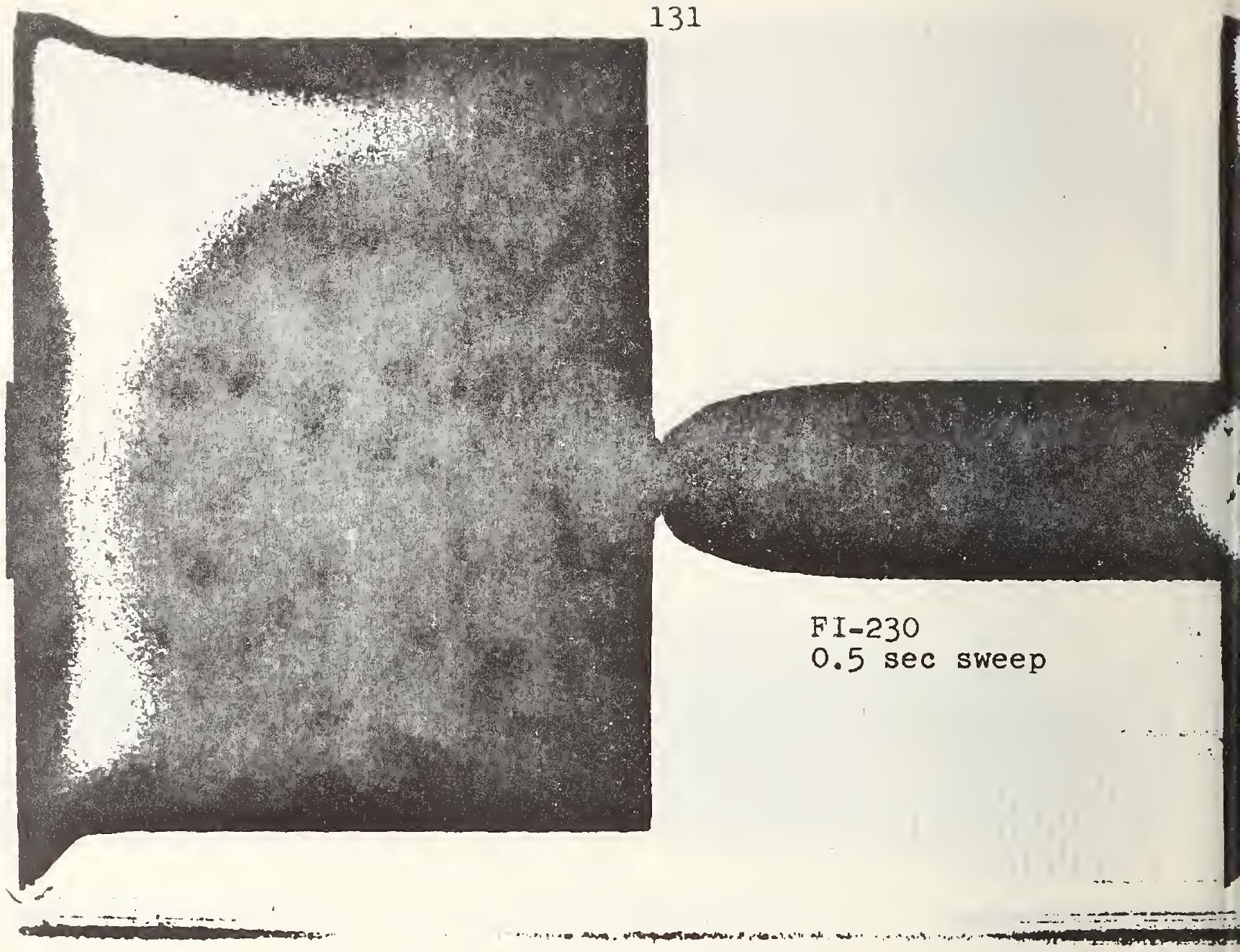




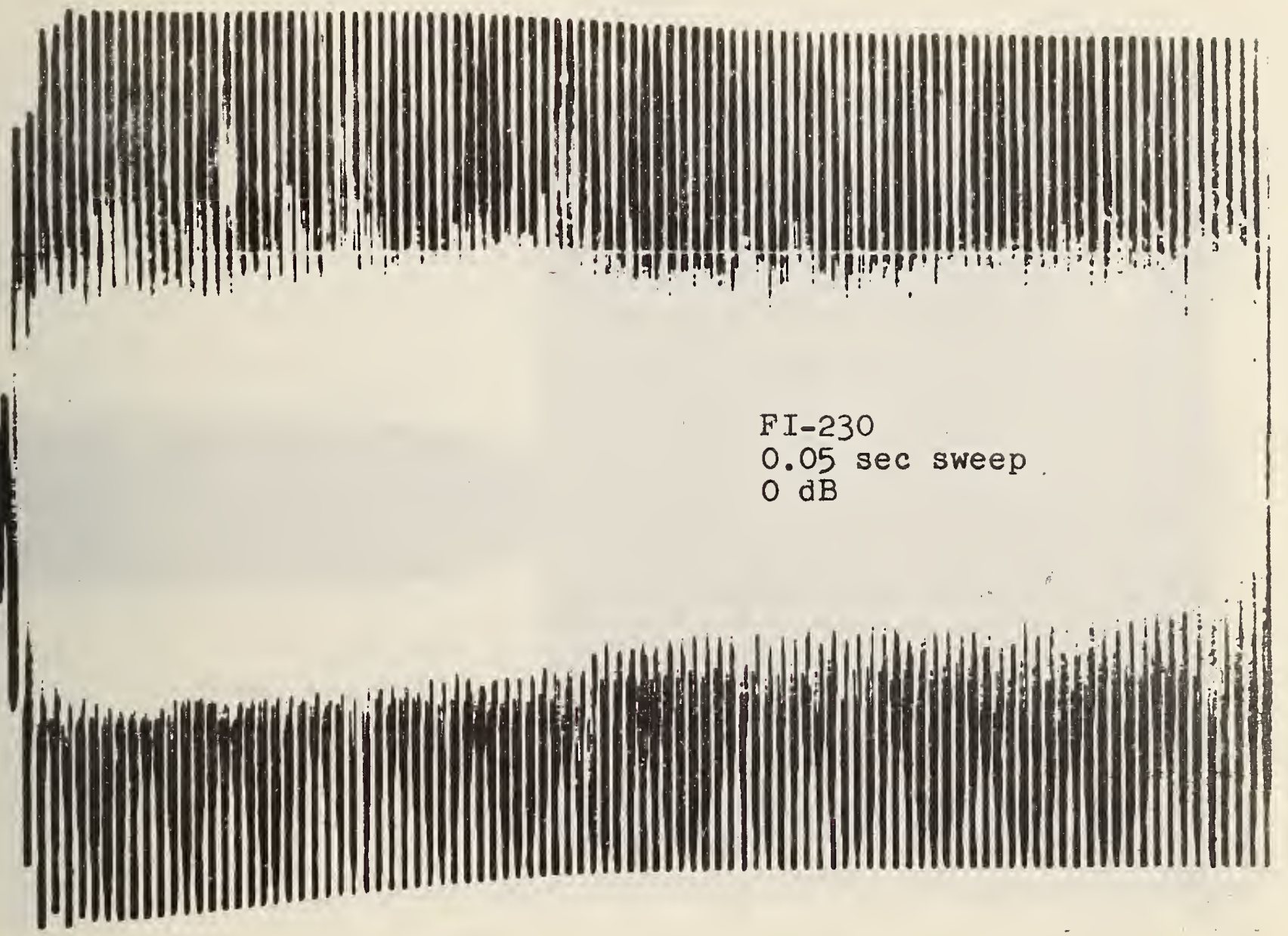




\section{0}

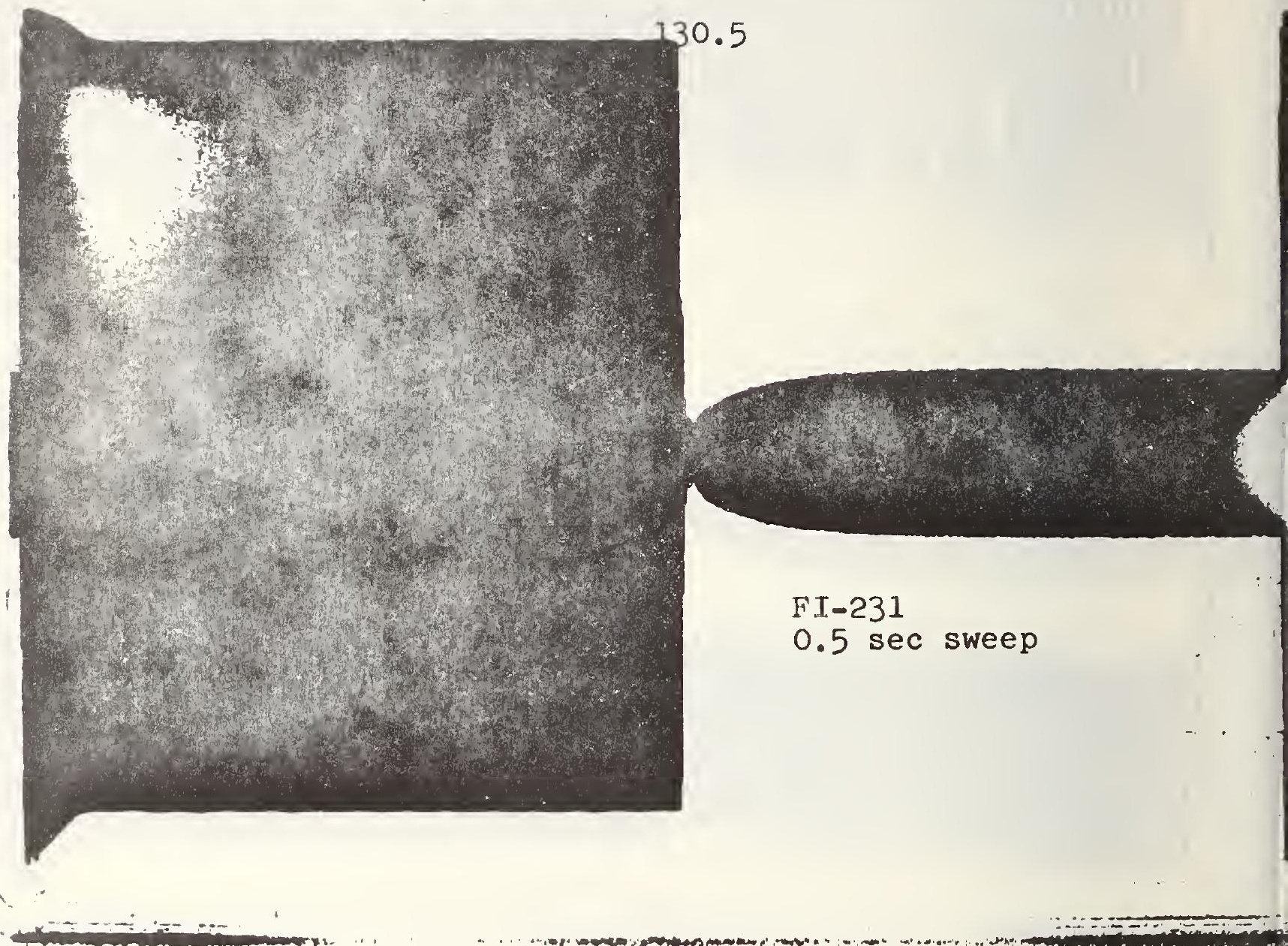




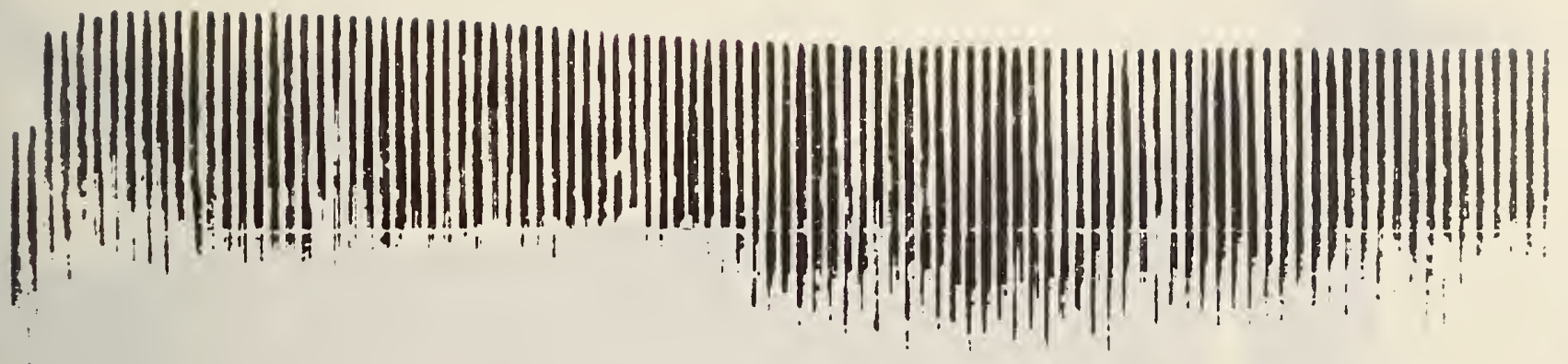

$$
\begin{aligned}
& \text { I }-231 \\
& 0.05 \text { sec sweep } \\
& \text { OdB }
\end{aligned}
$$

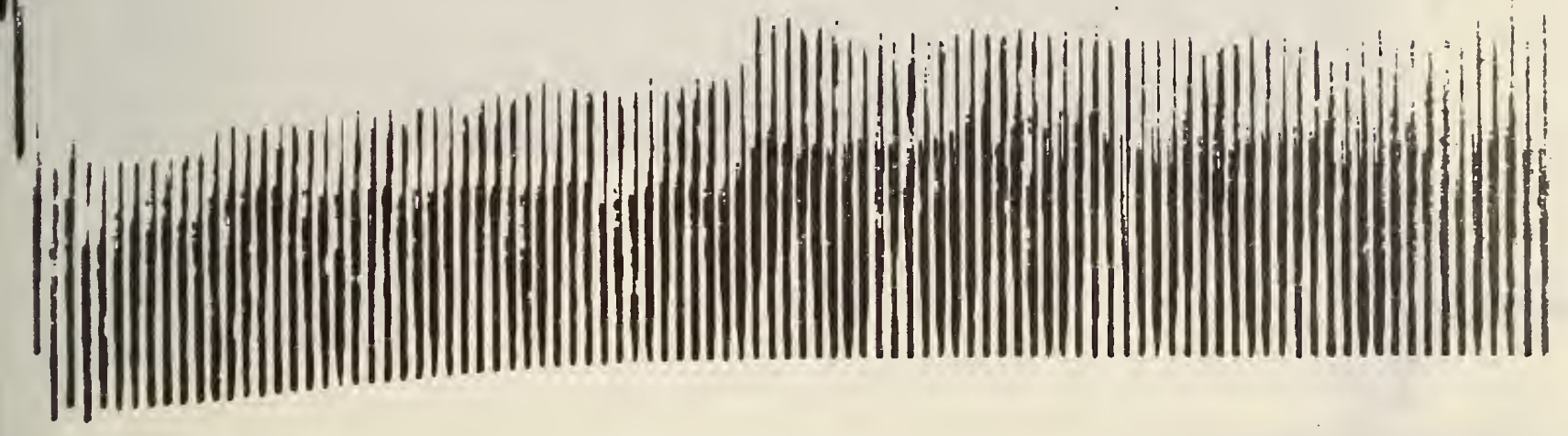




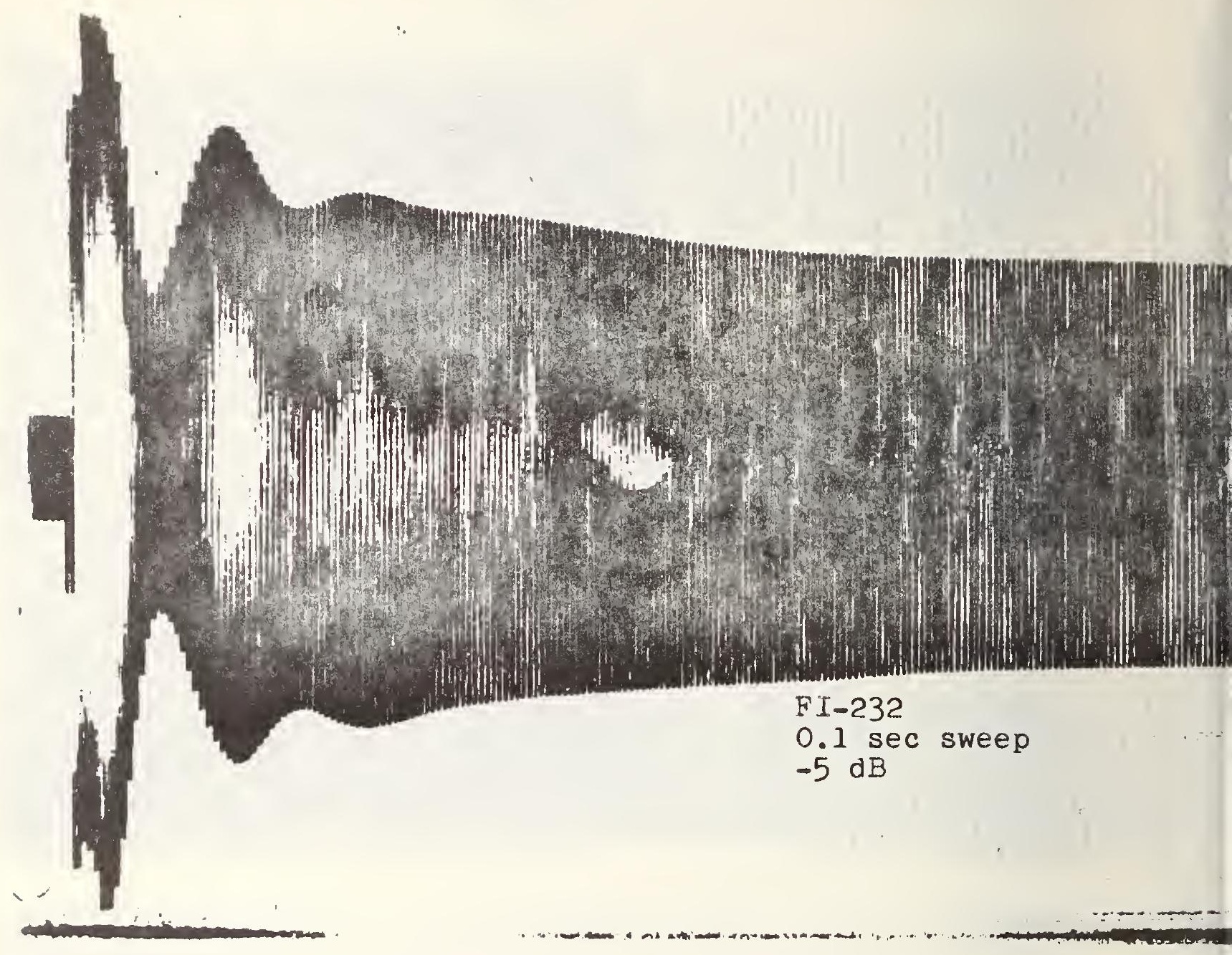


124

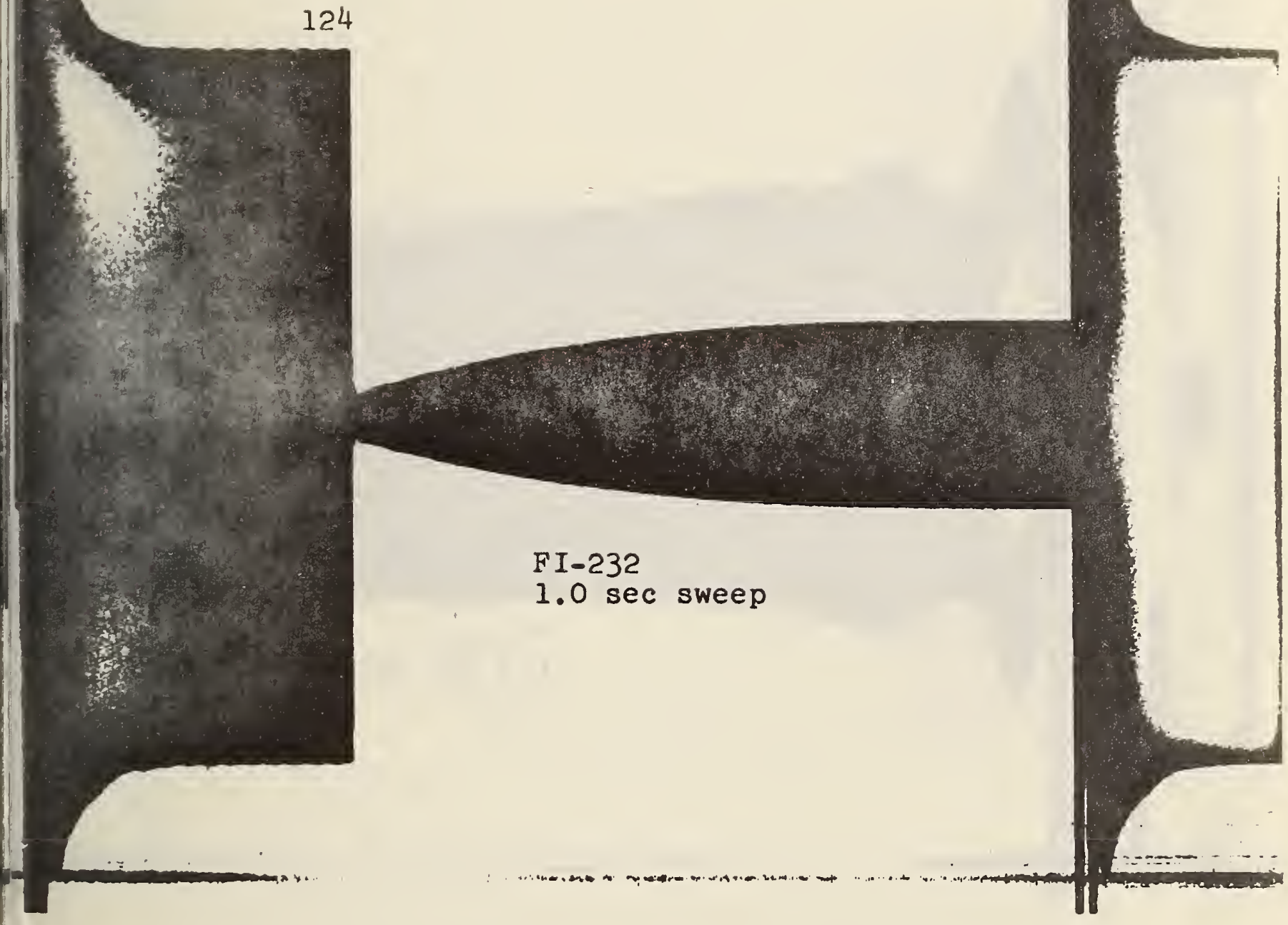




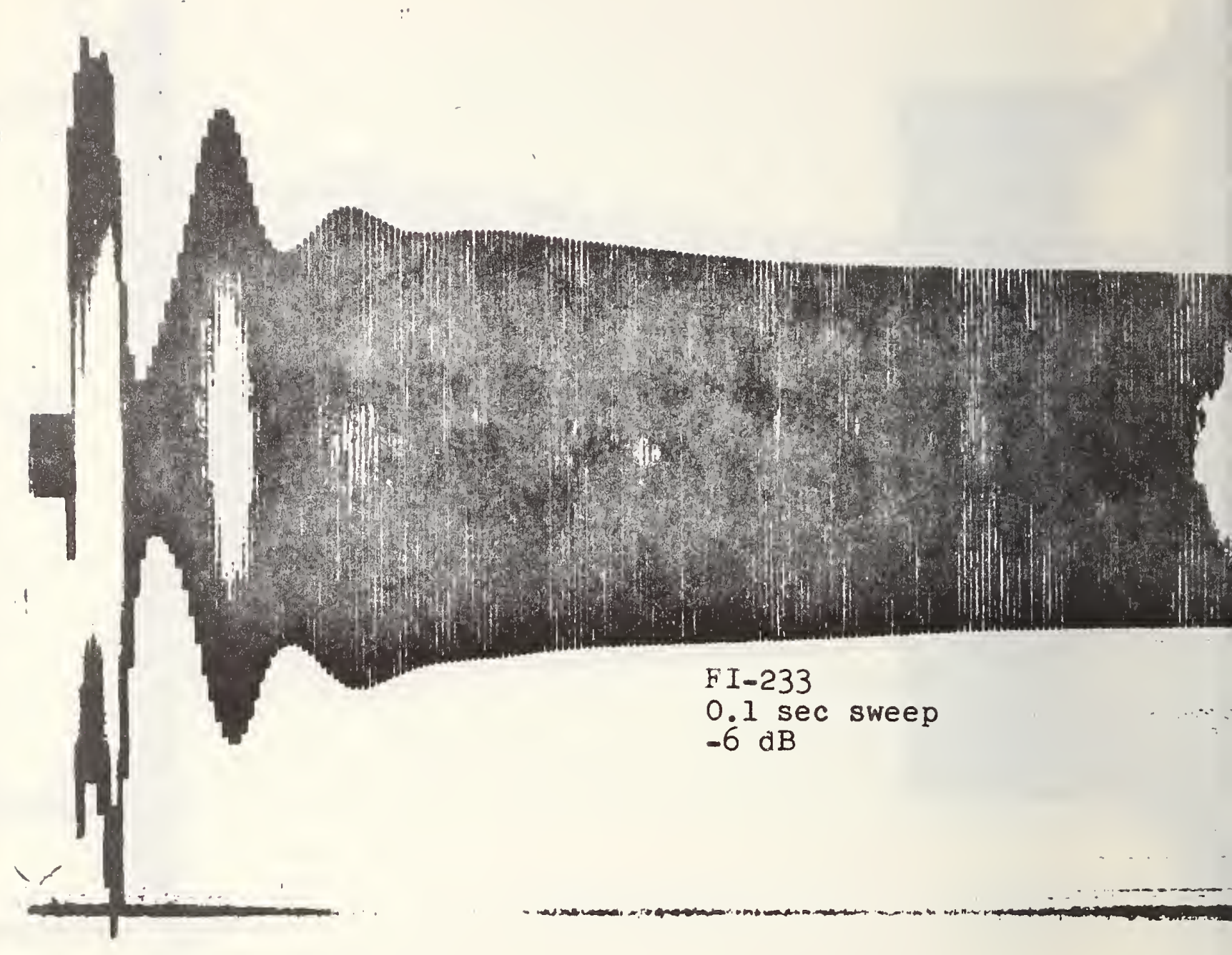


123.5

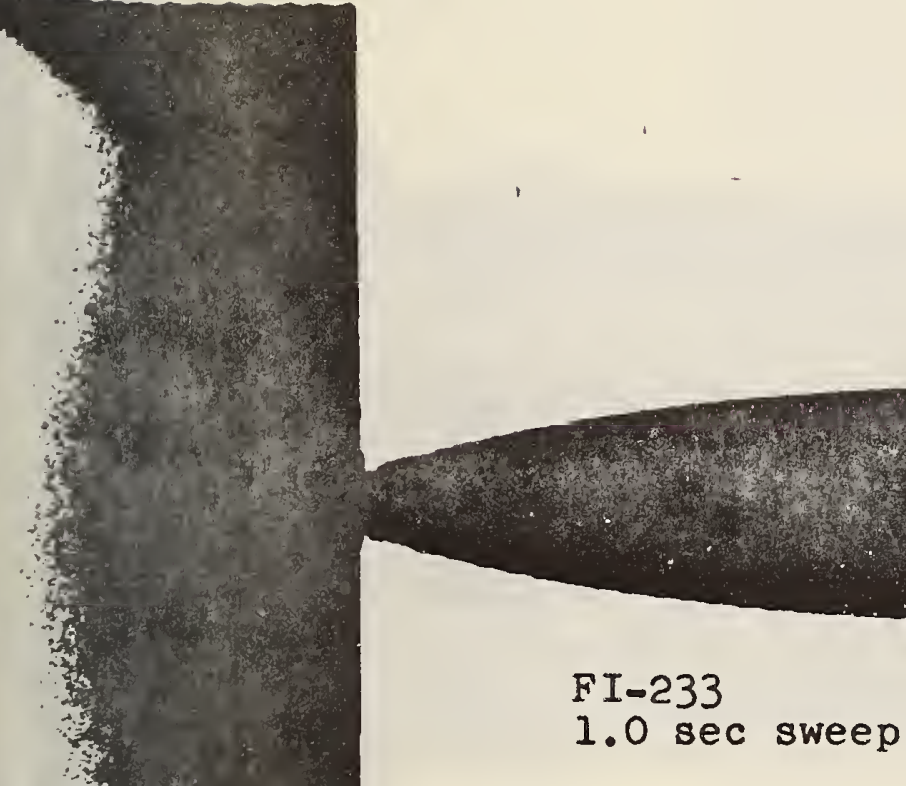

1.0 sec sweep

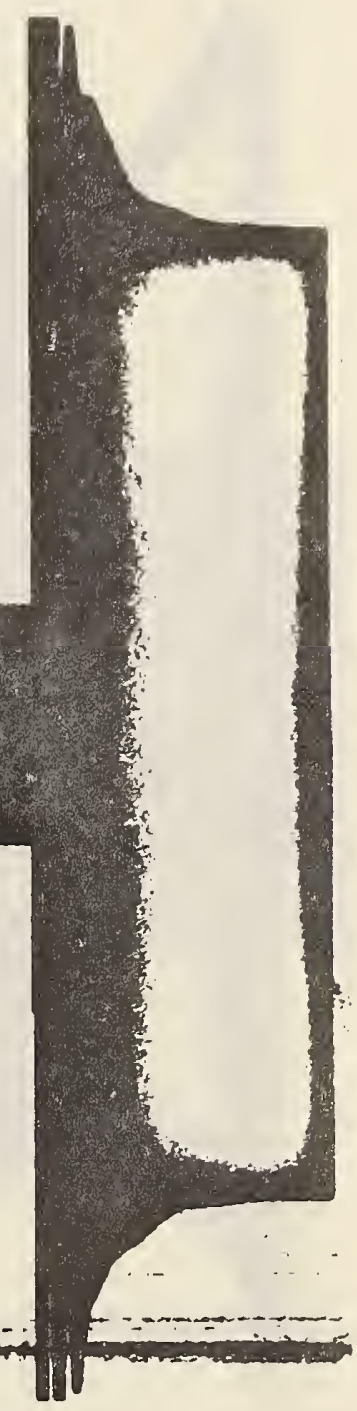




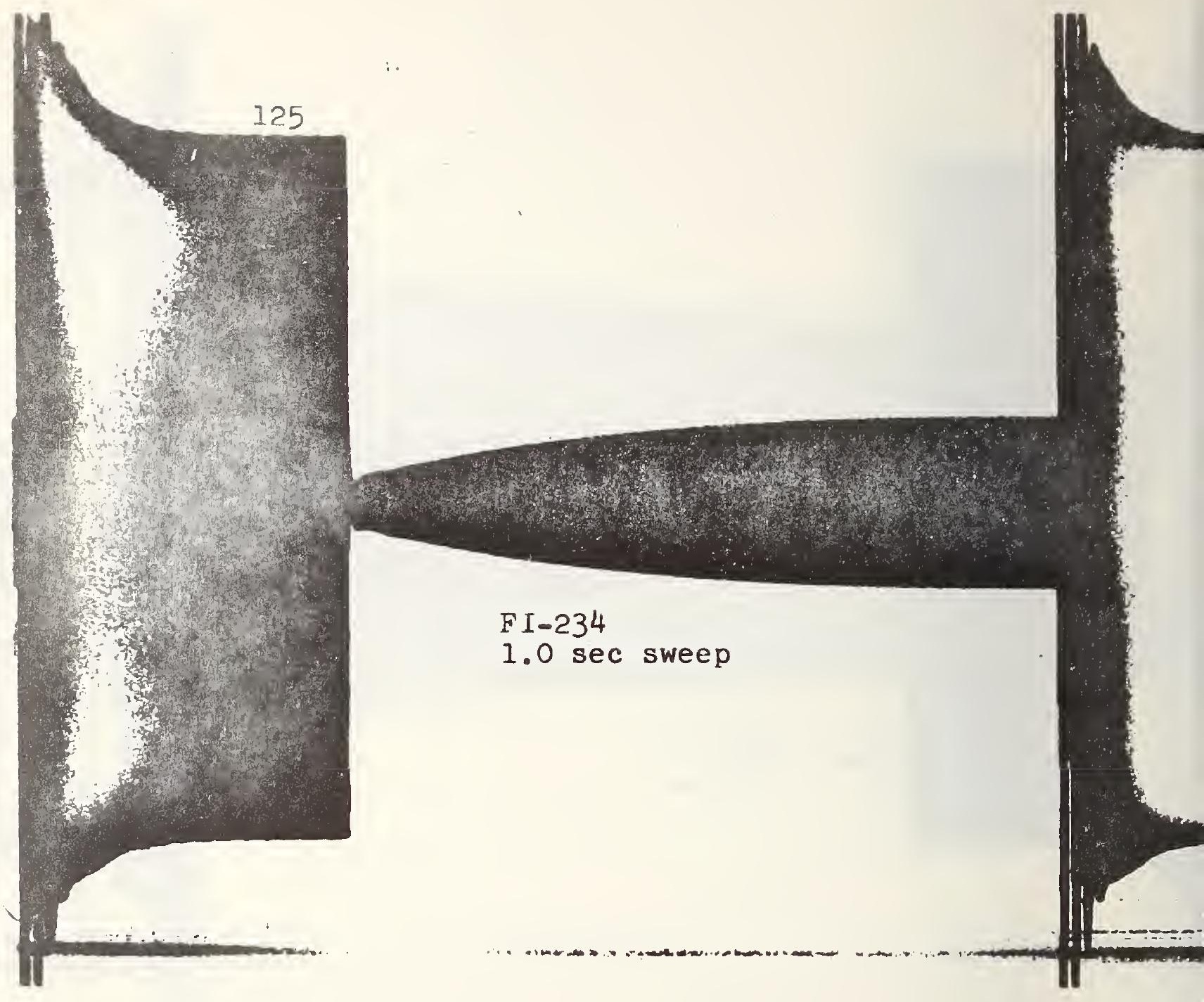




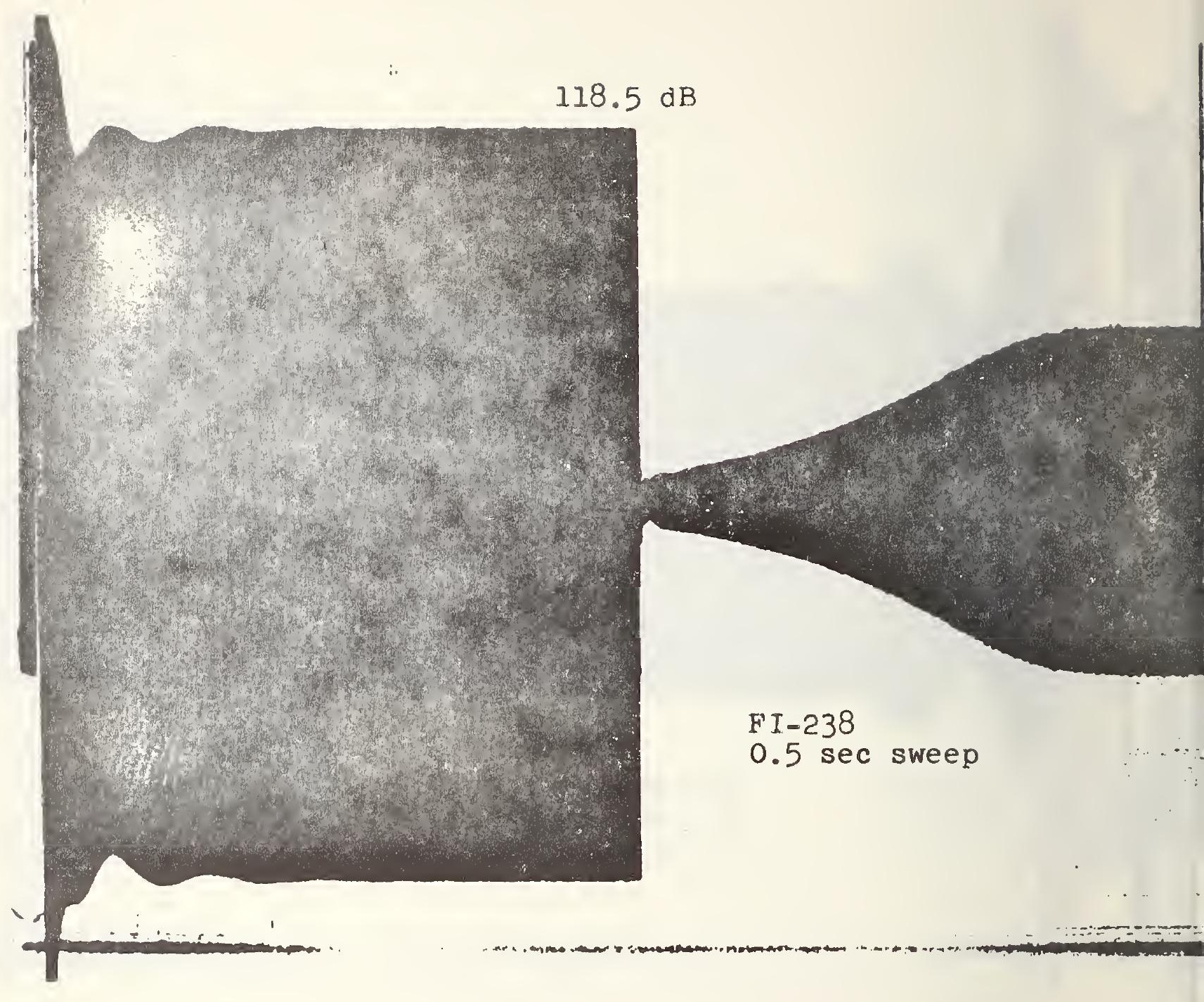




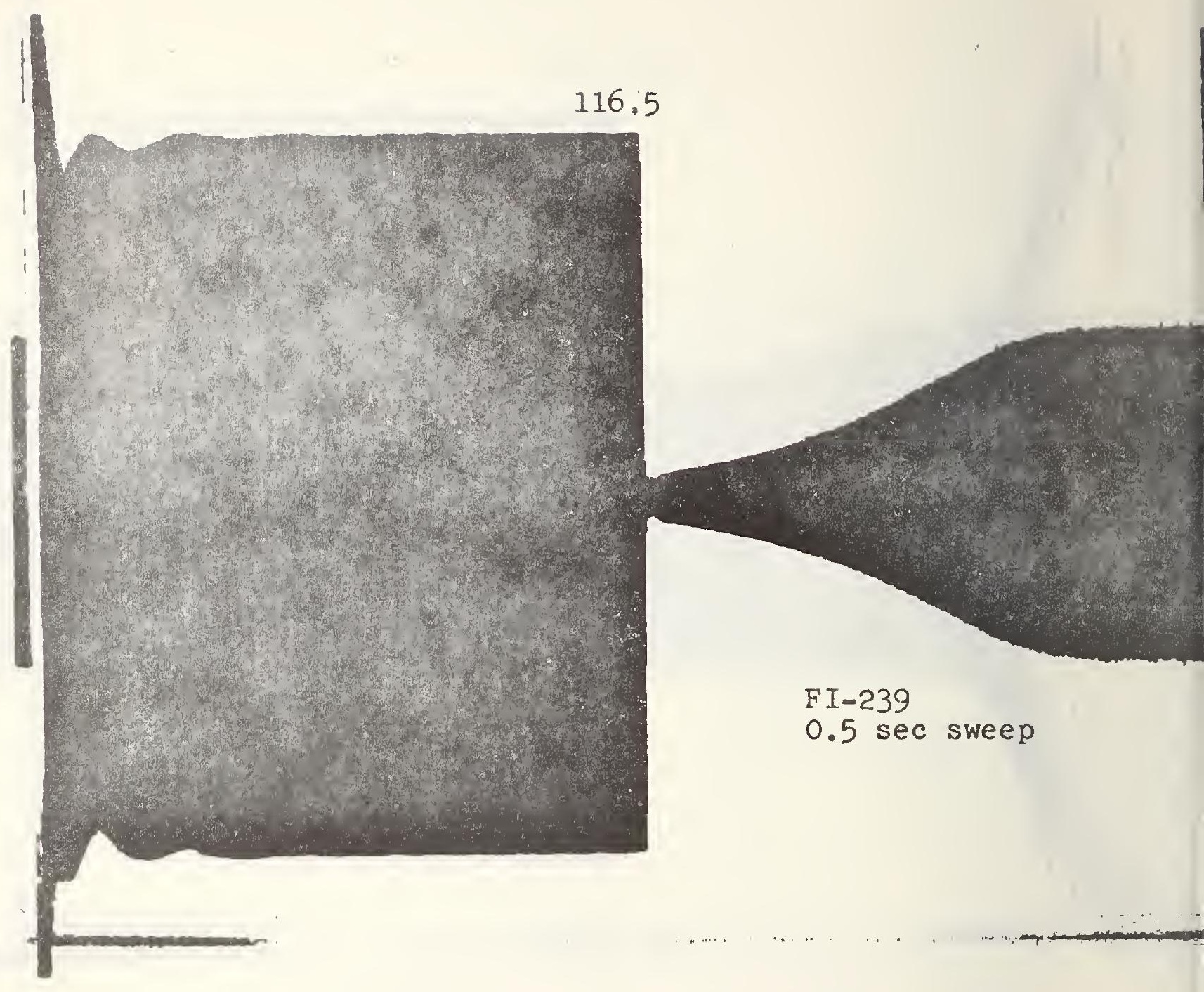




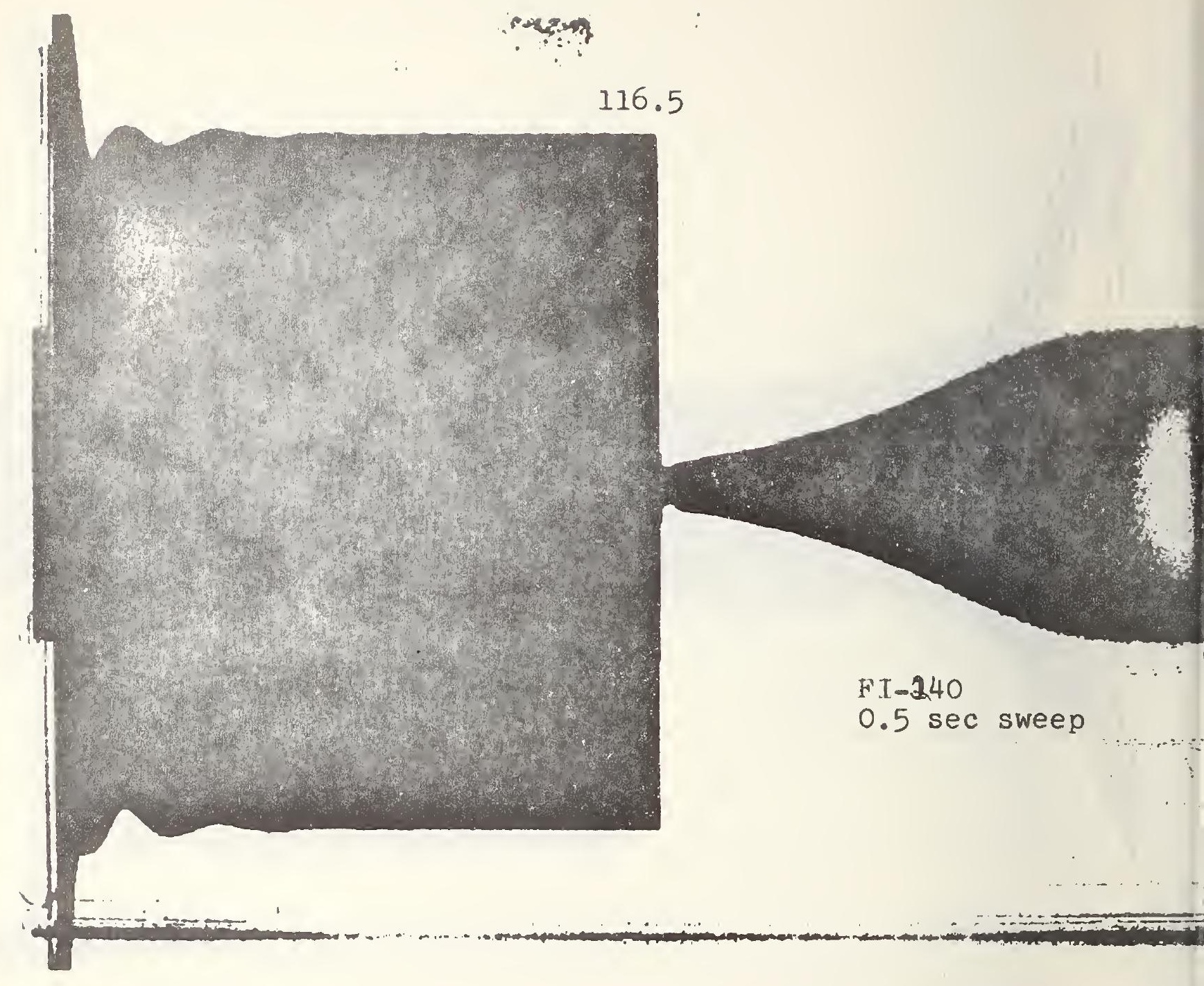




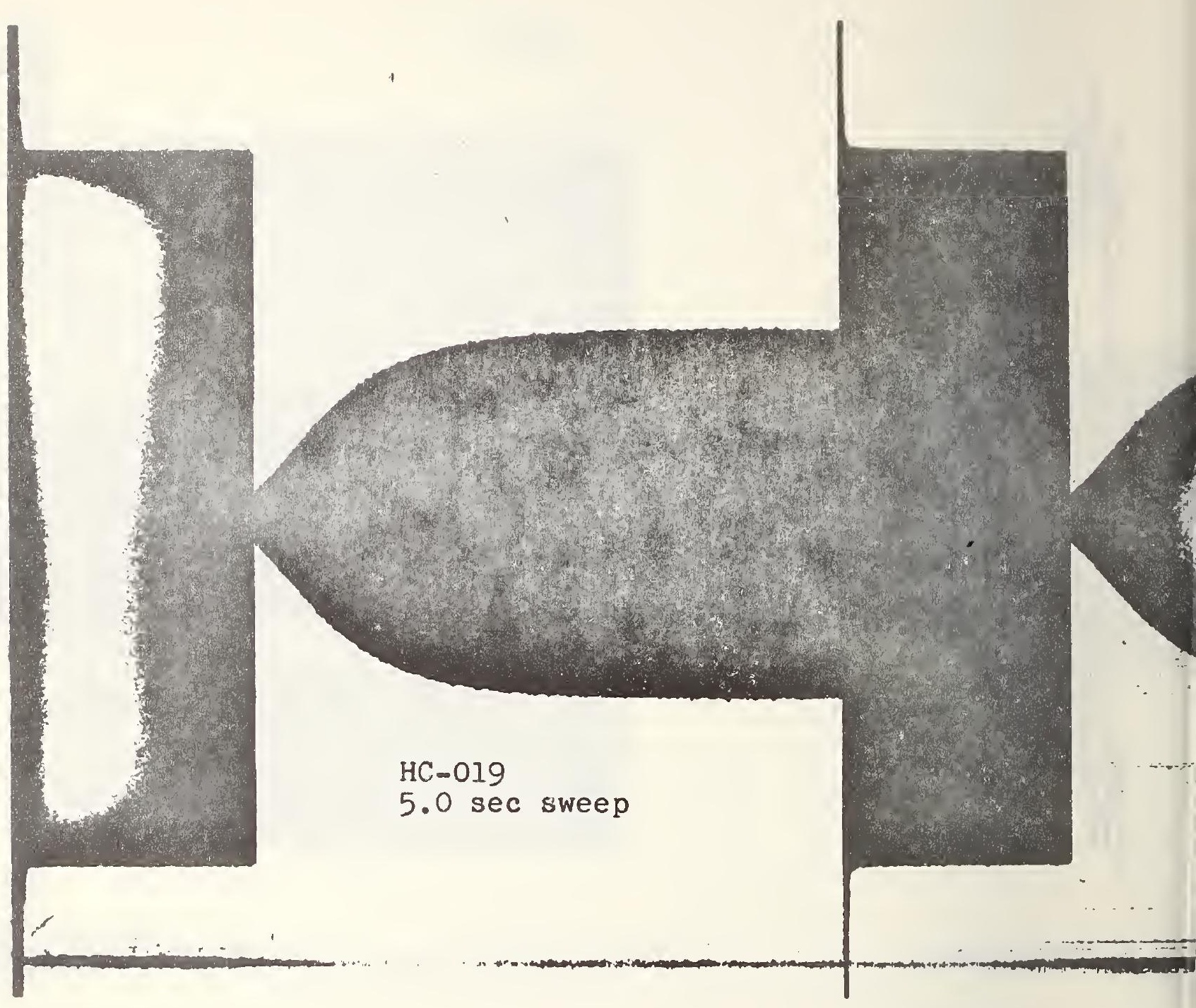




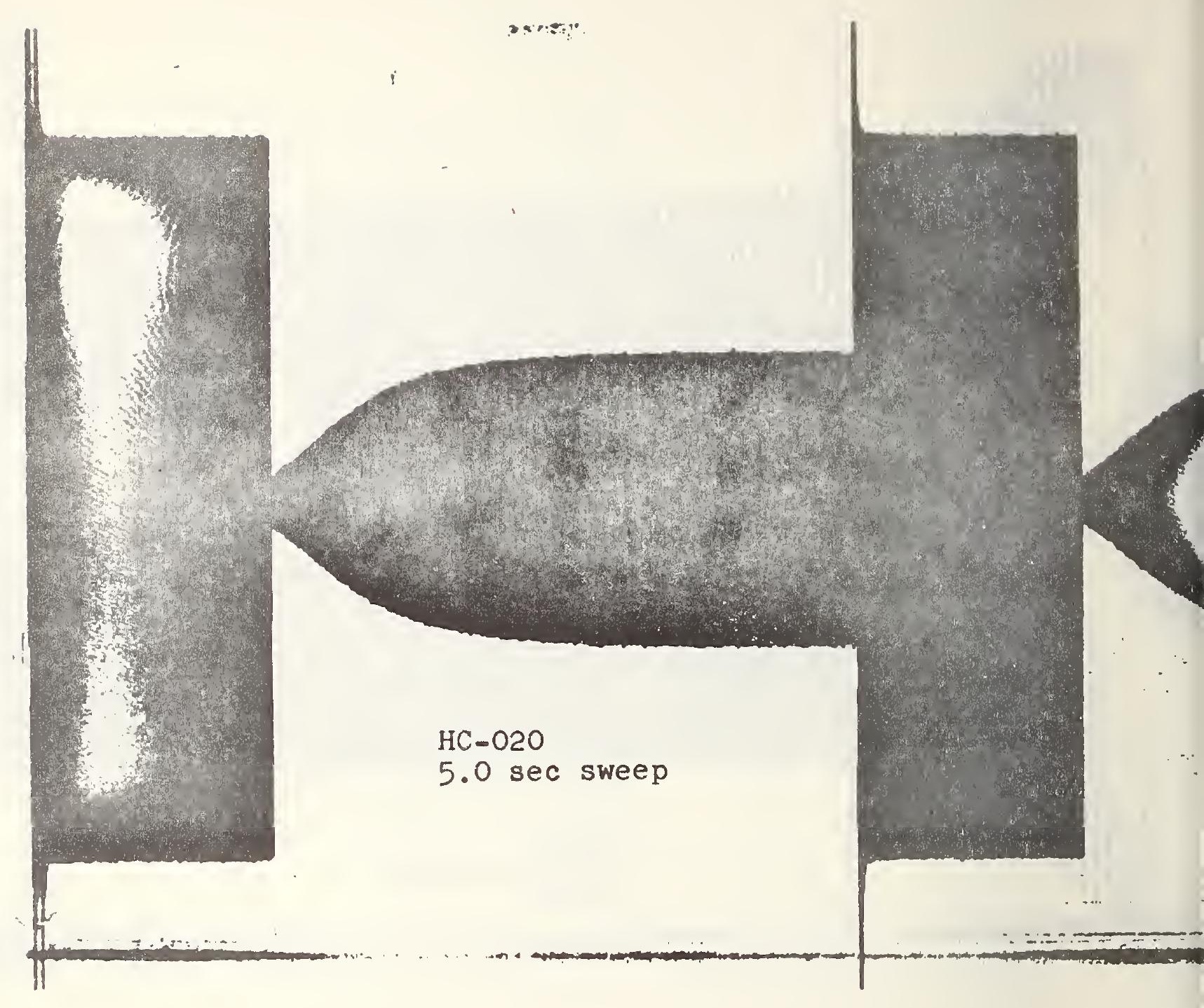


$\| !|! ! !||| \mid$

.

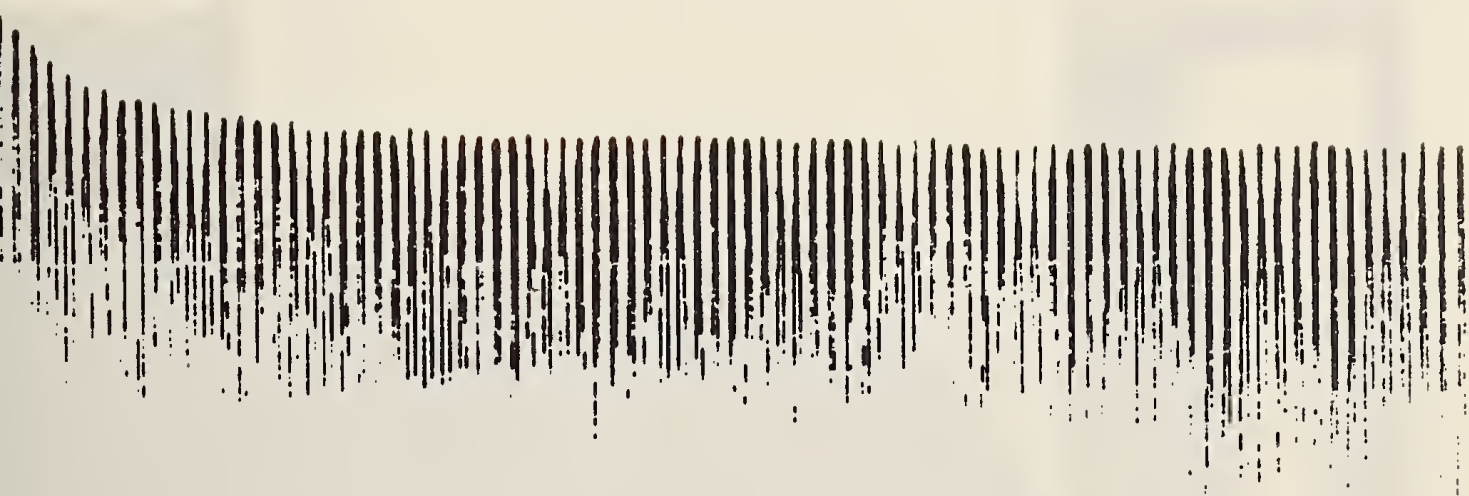
if Hc-02O

0.05 sec sweep $2-2 B-1 d B$ 


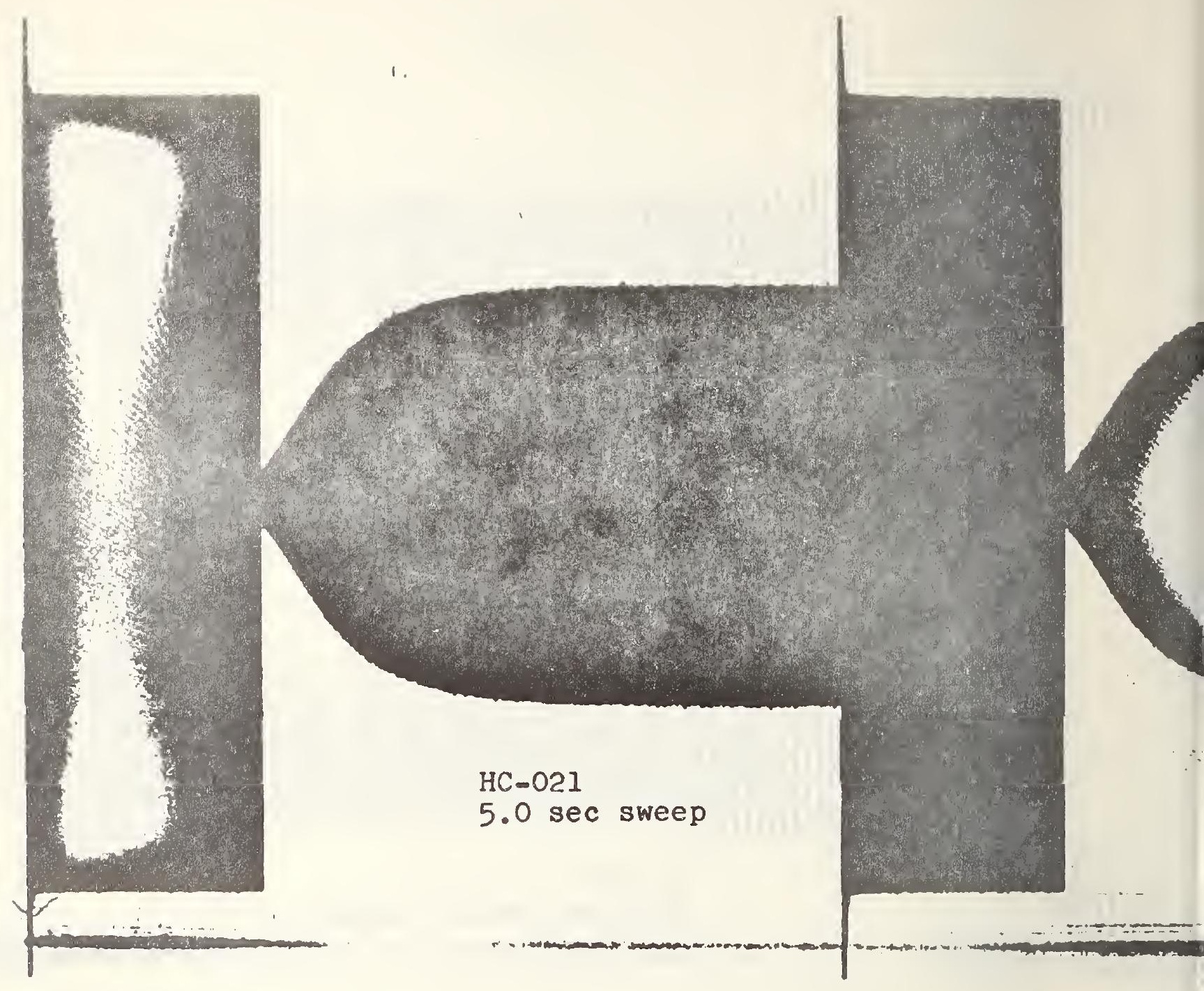




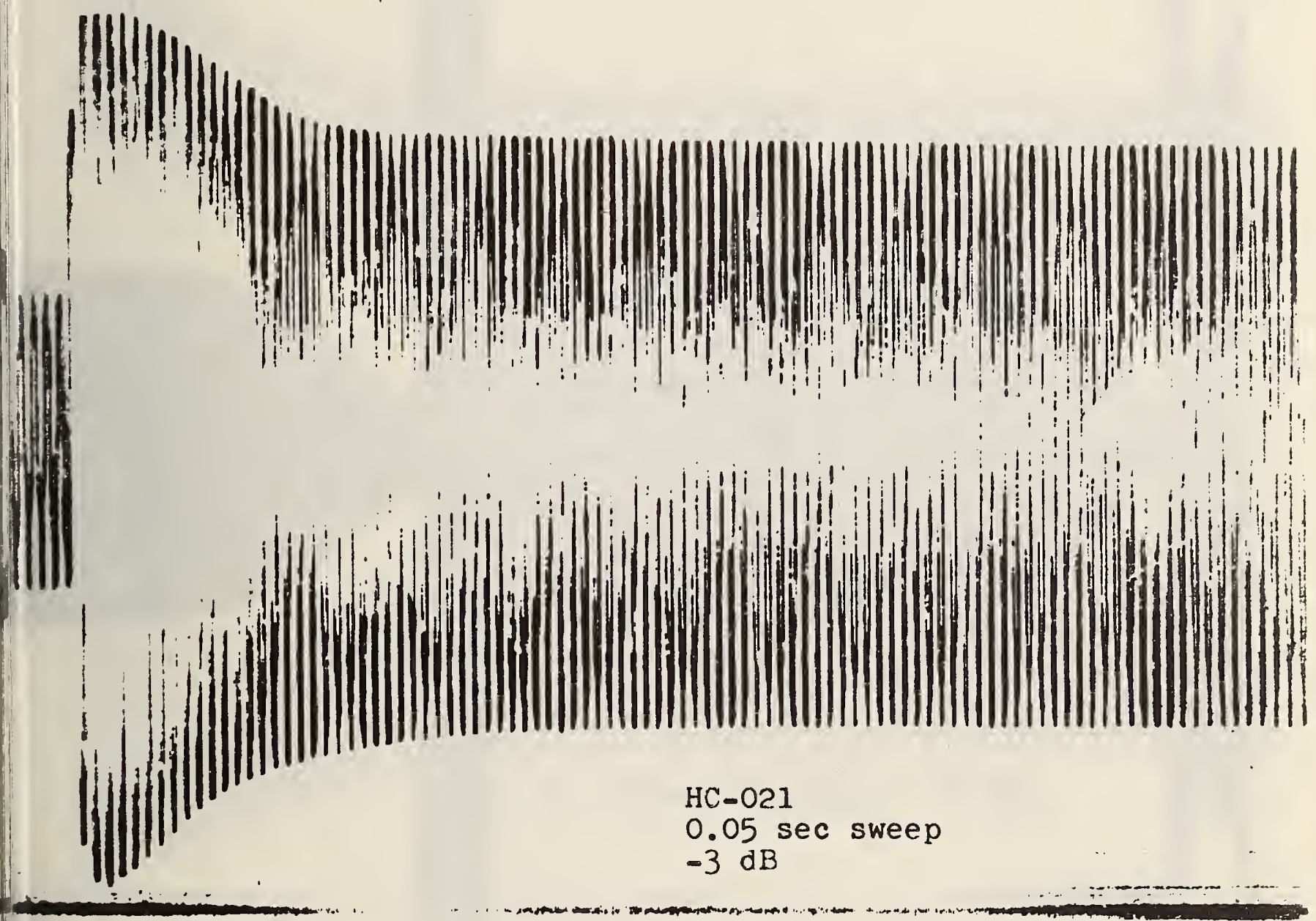




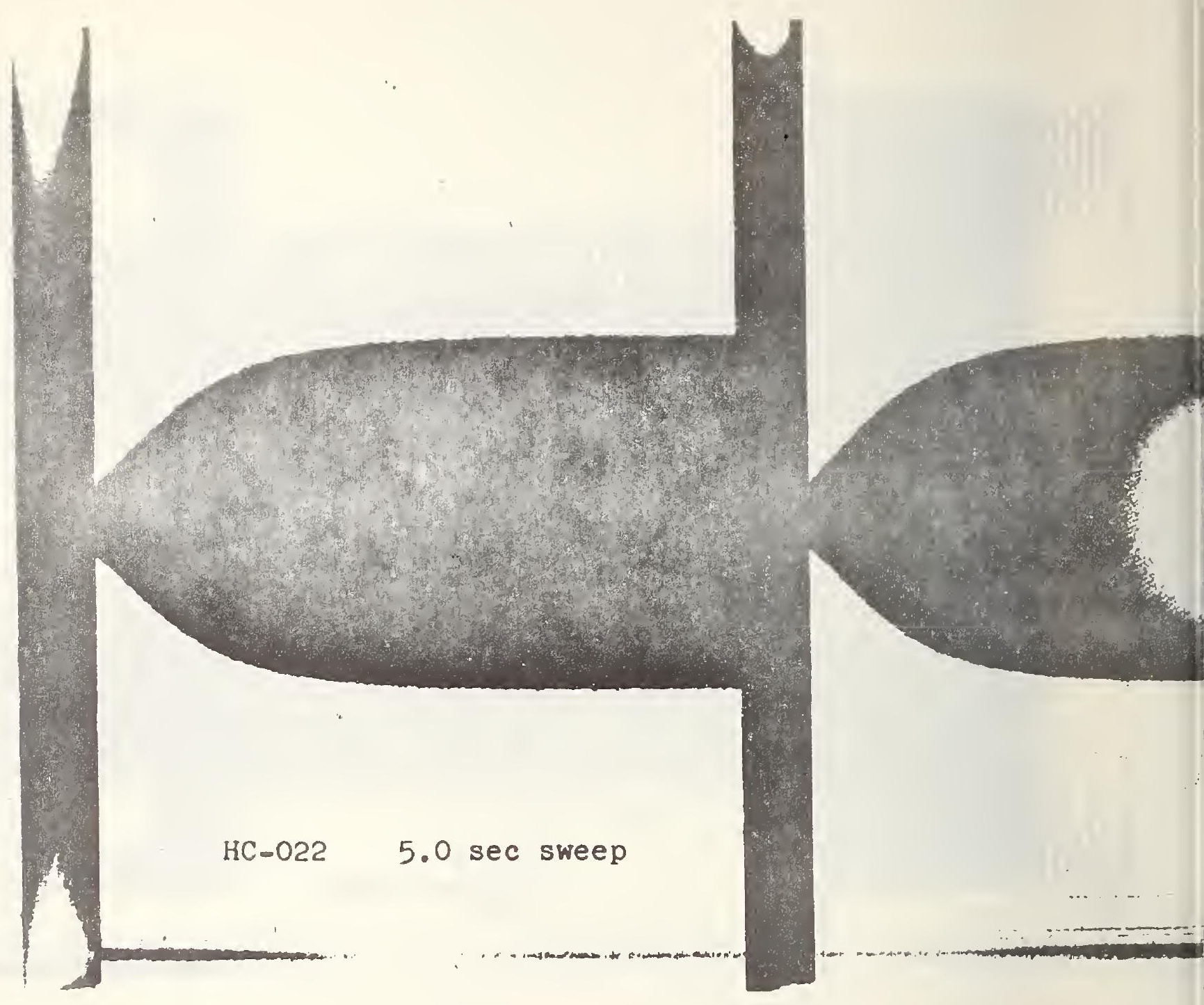




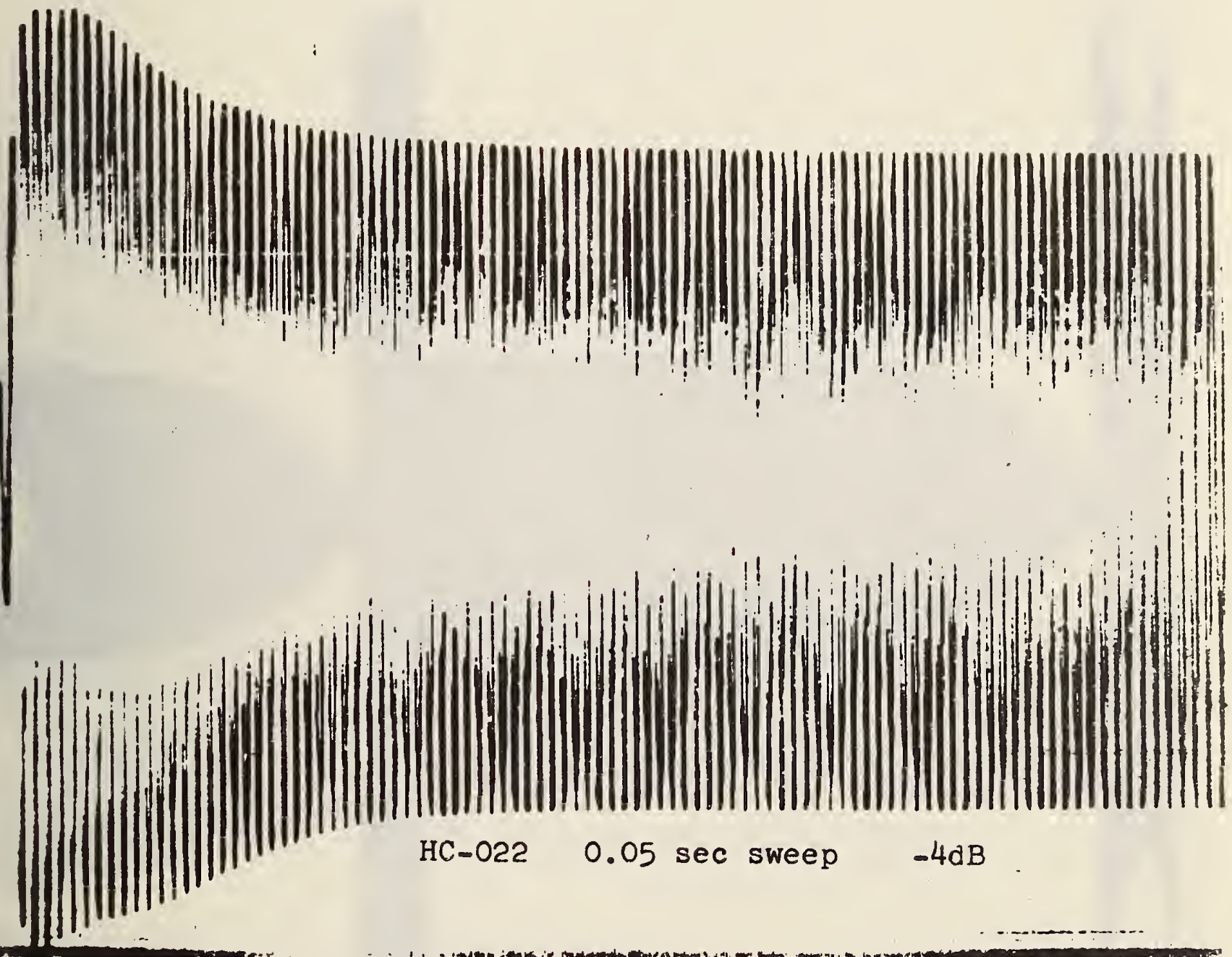




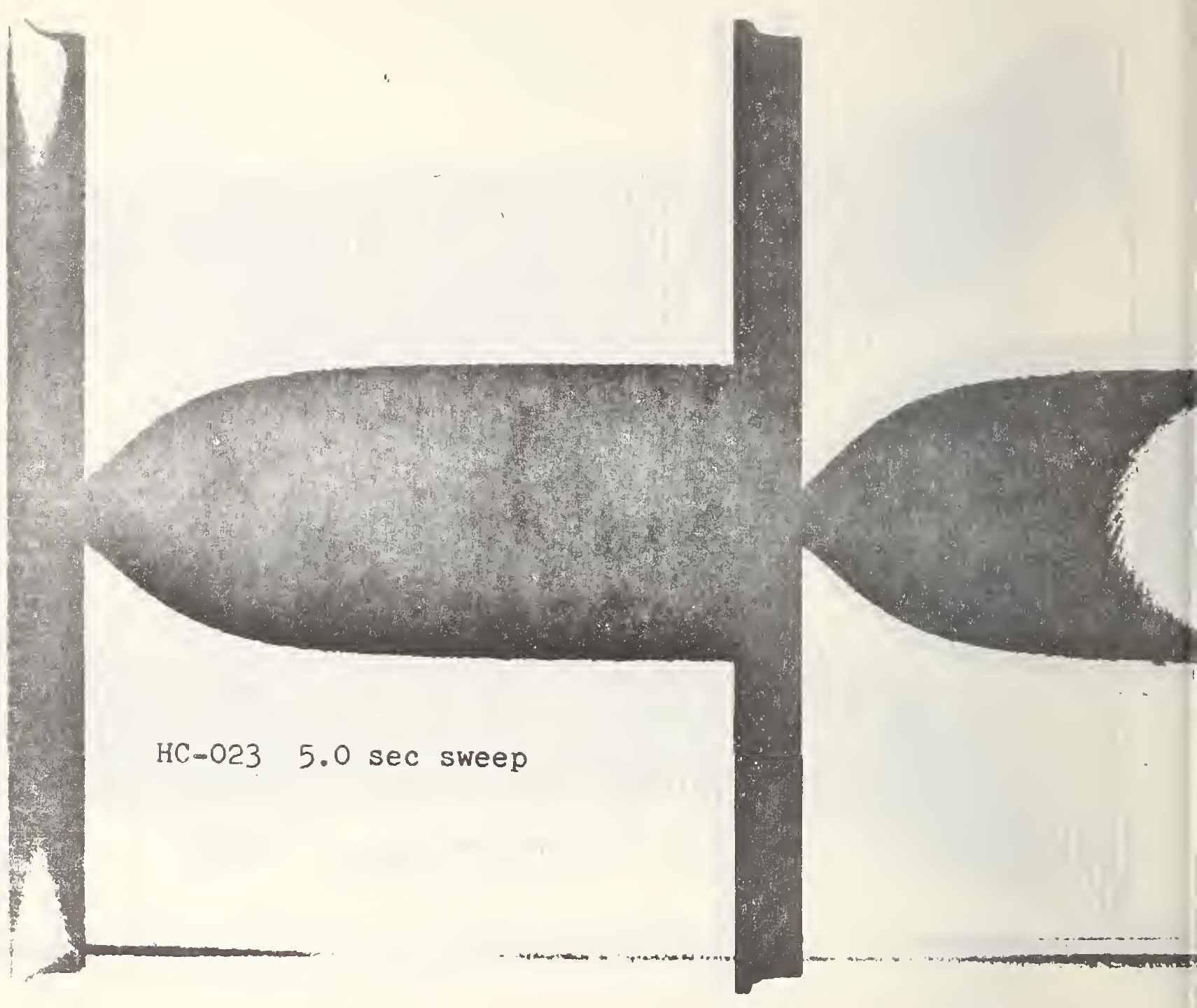




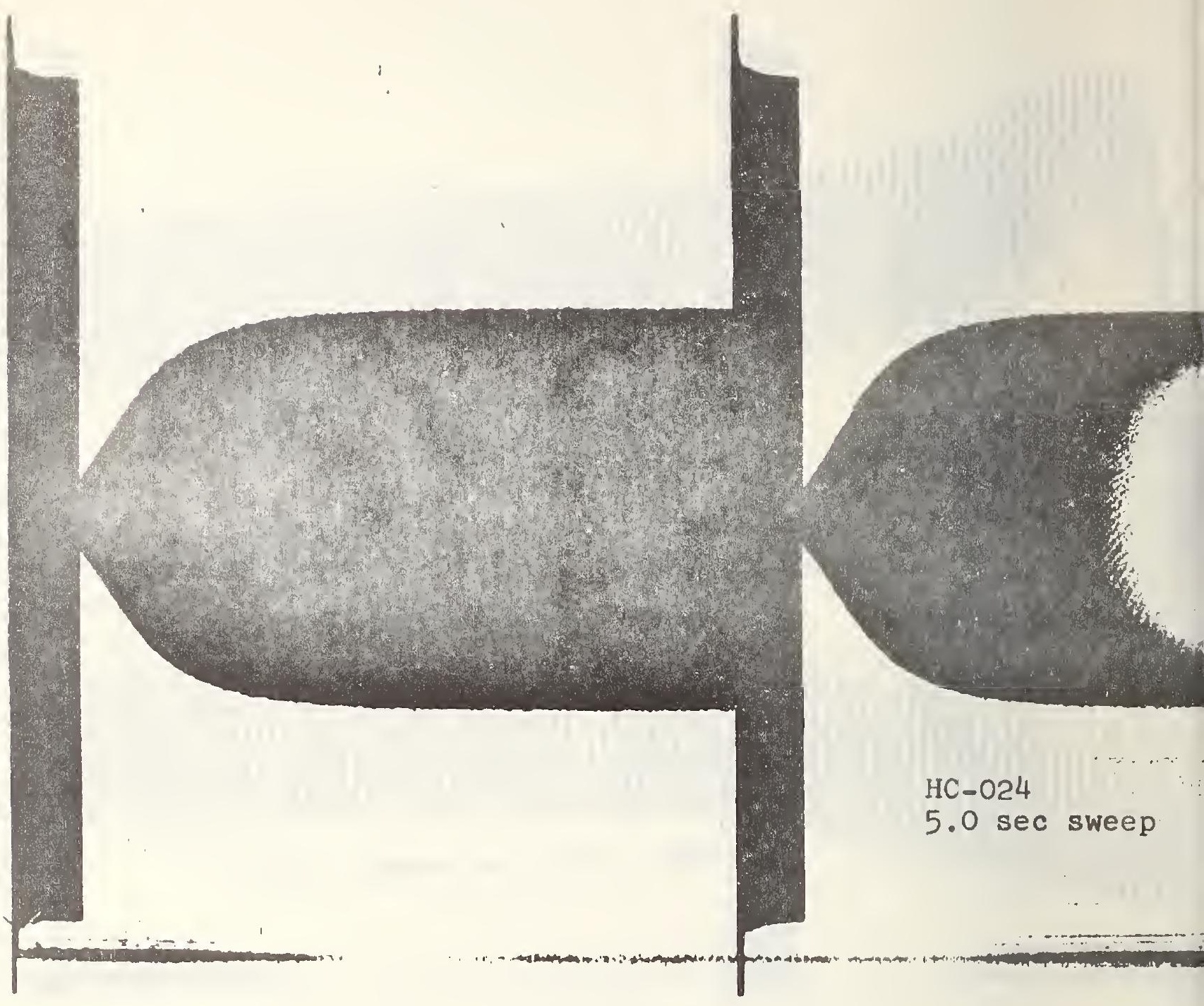




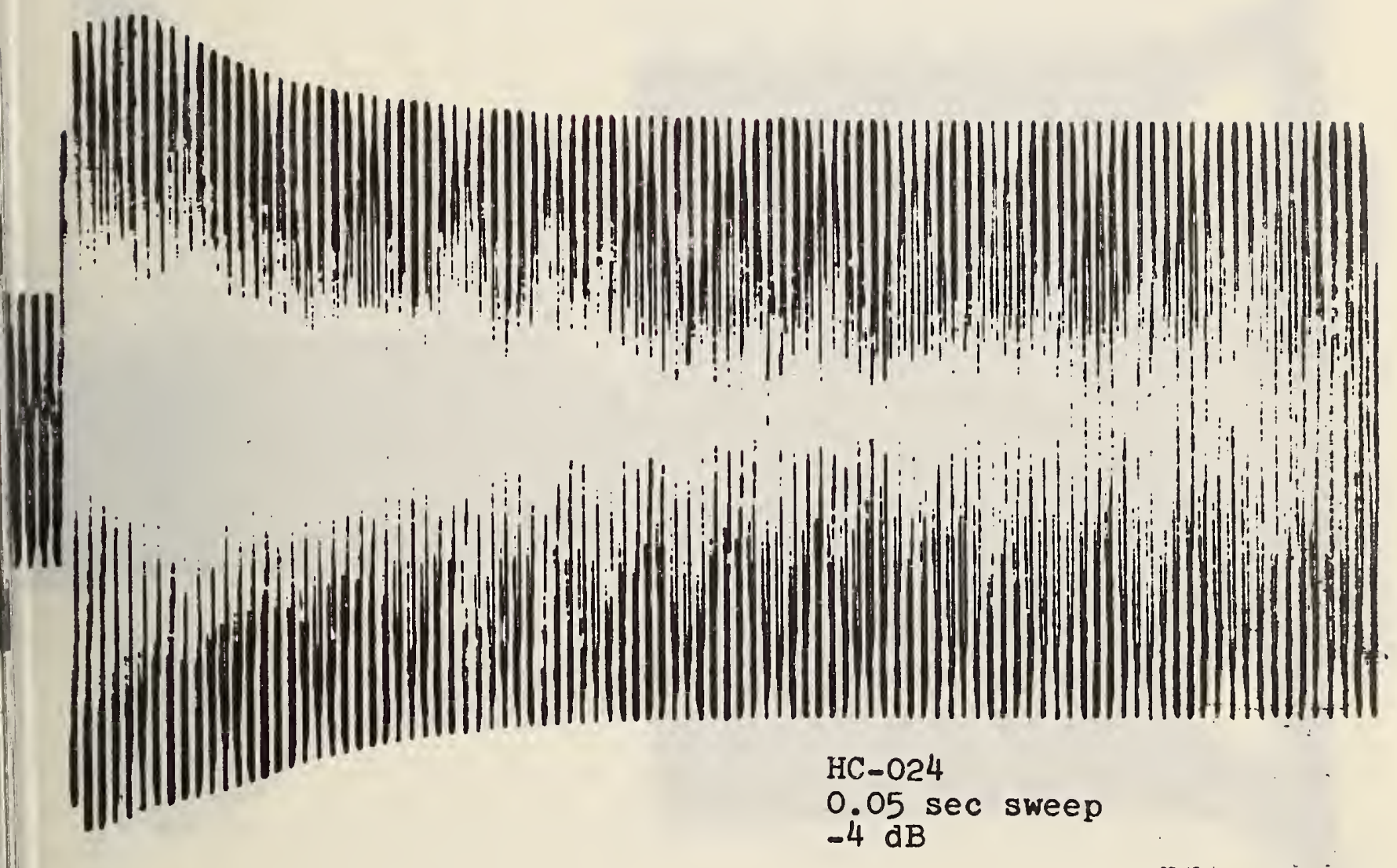




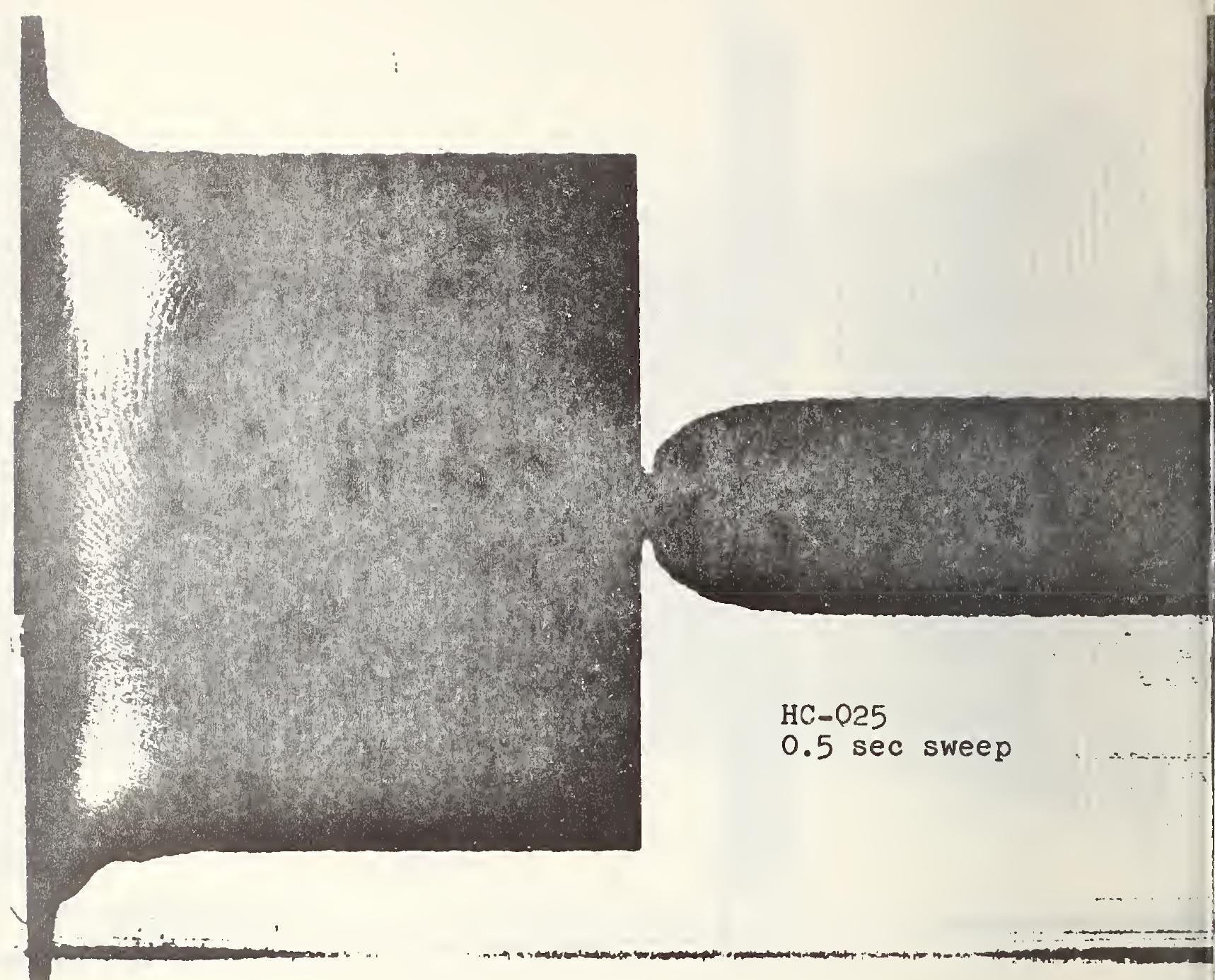




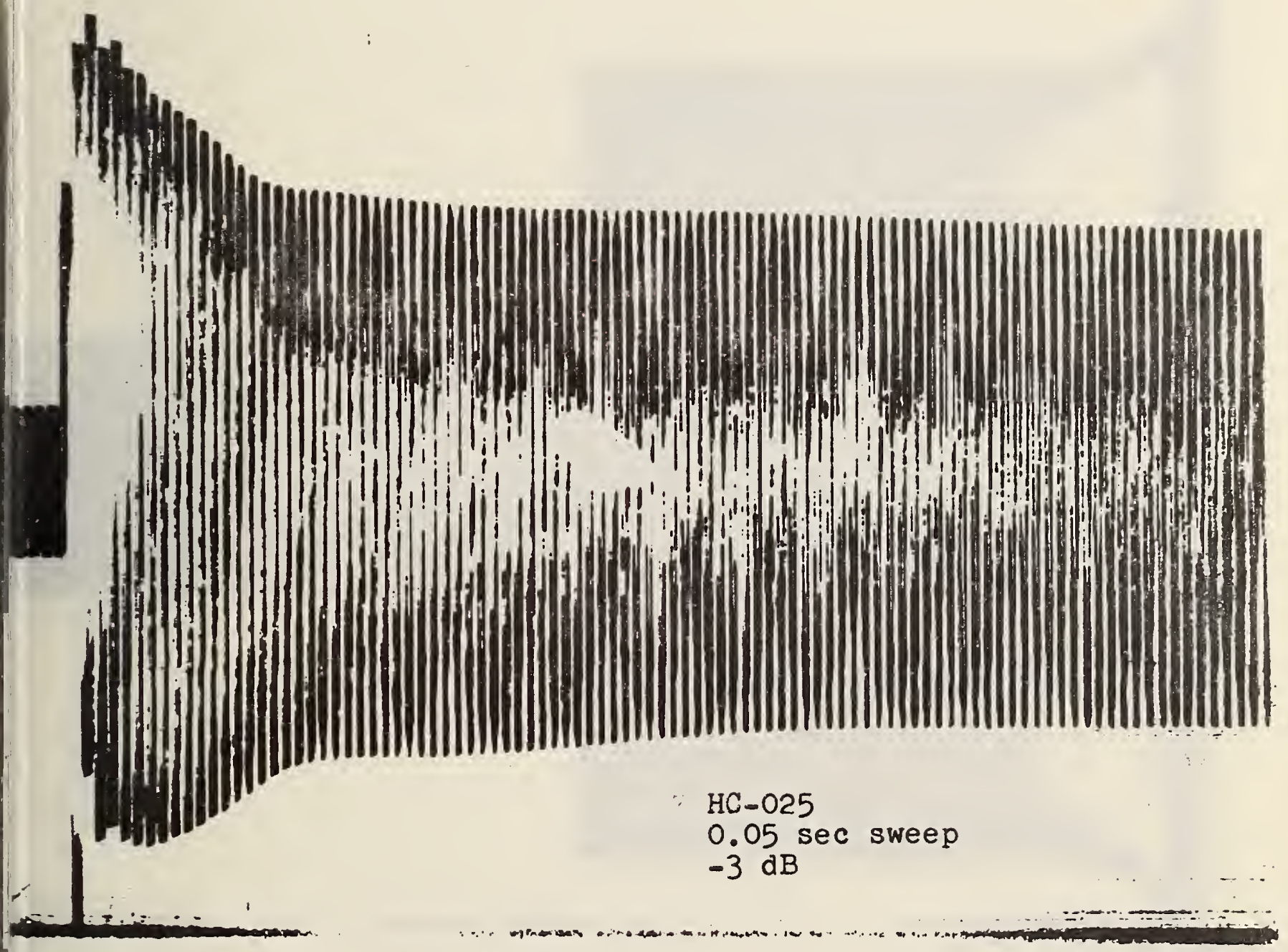




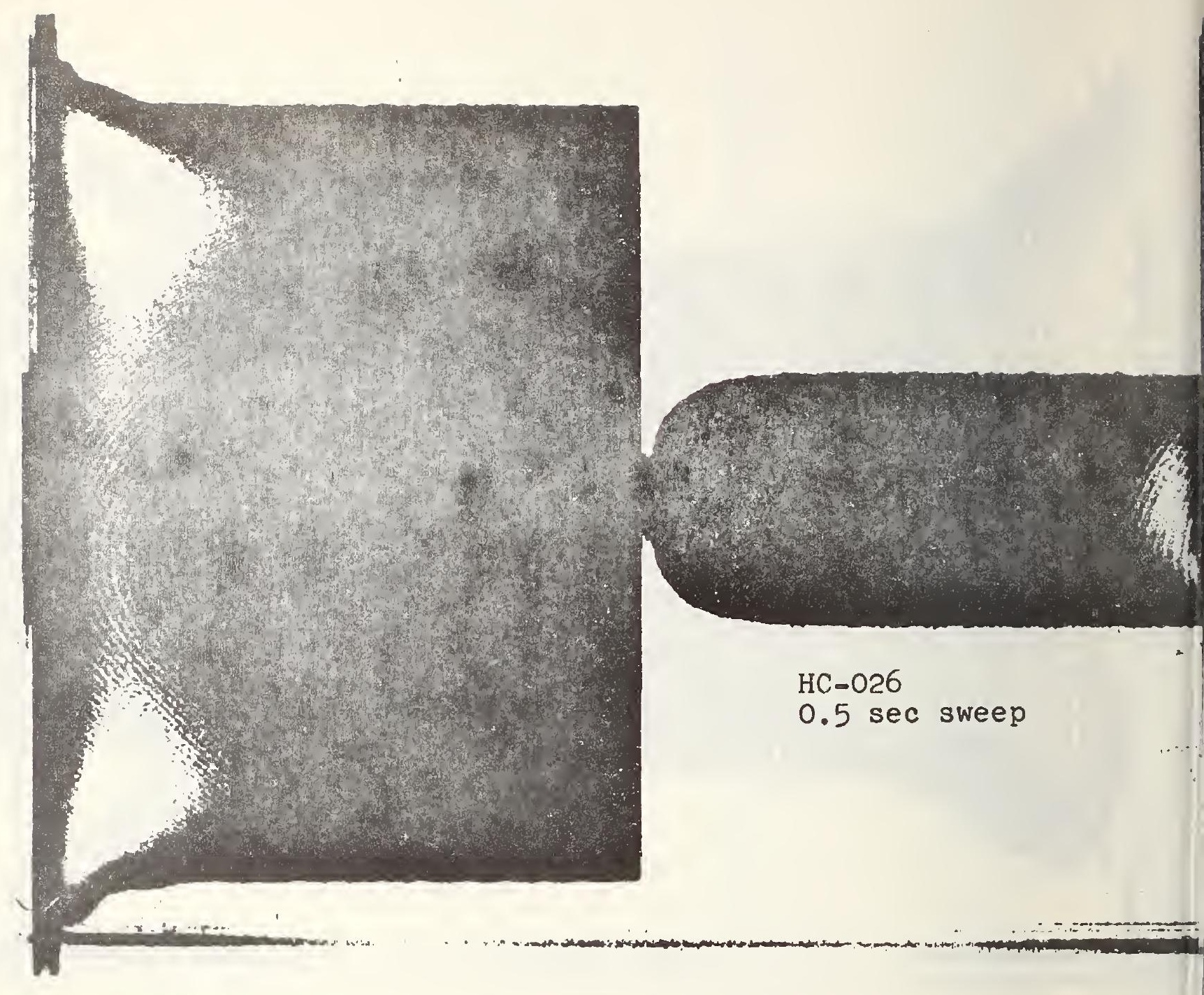




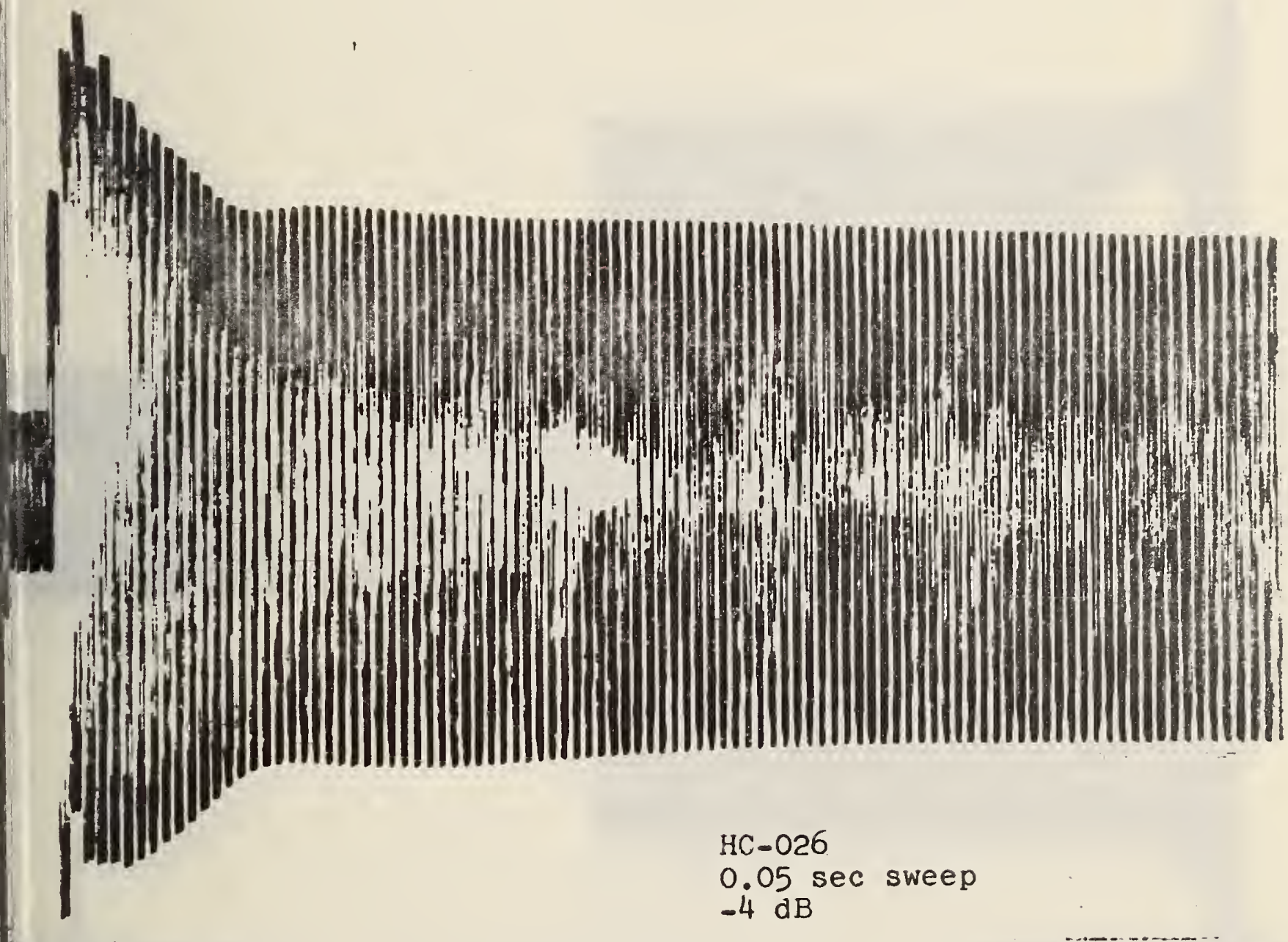




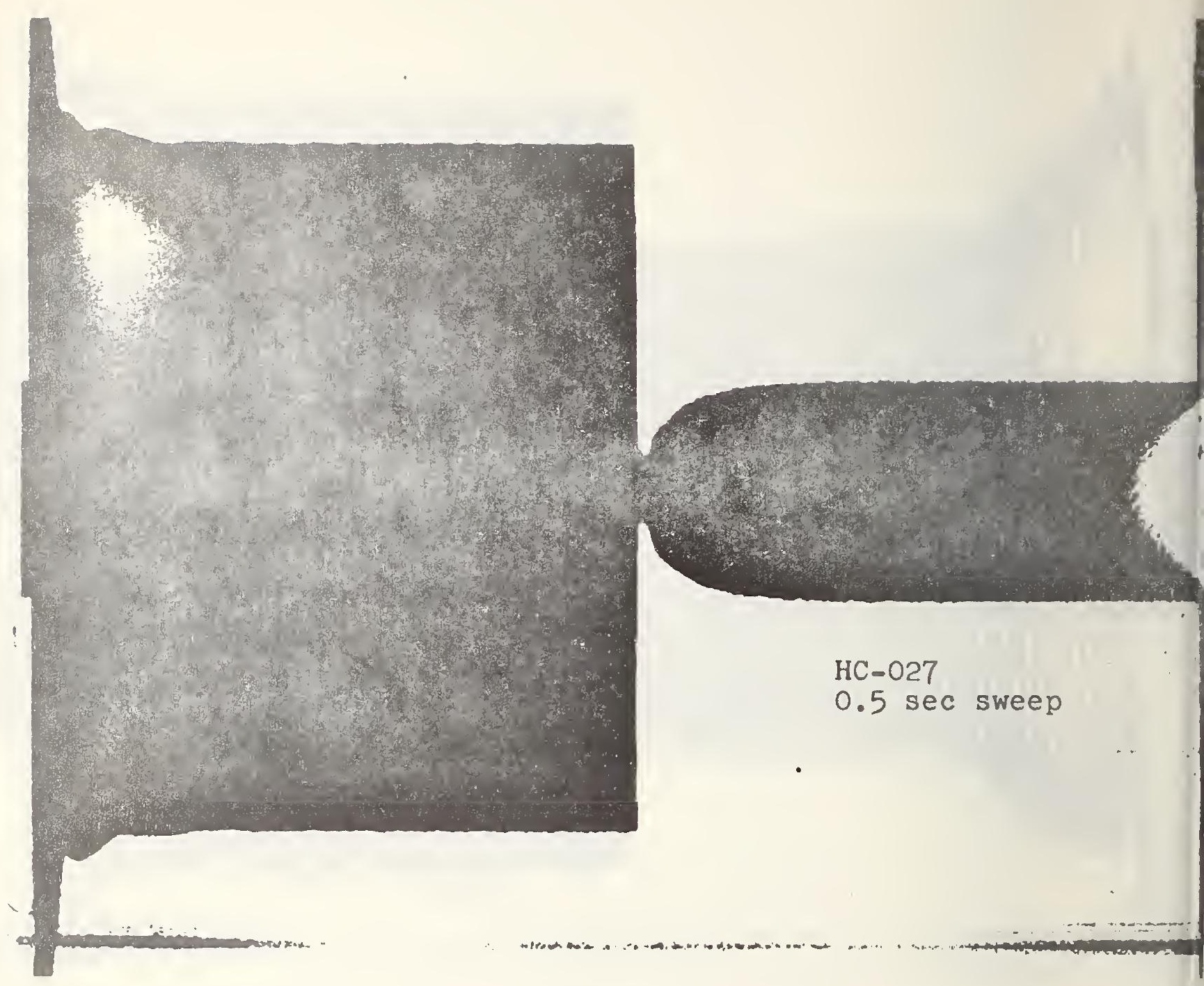




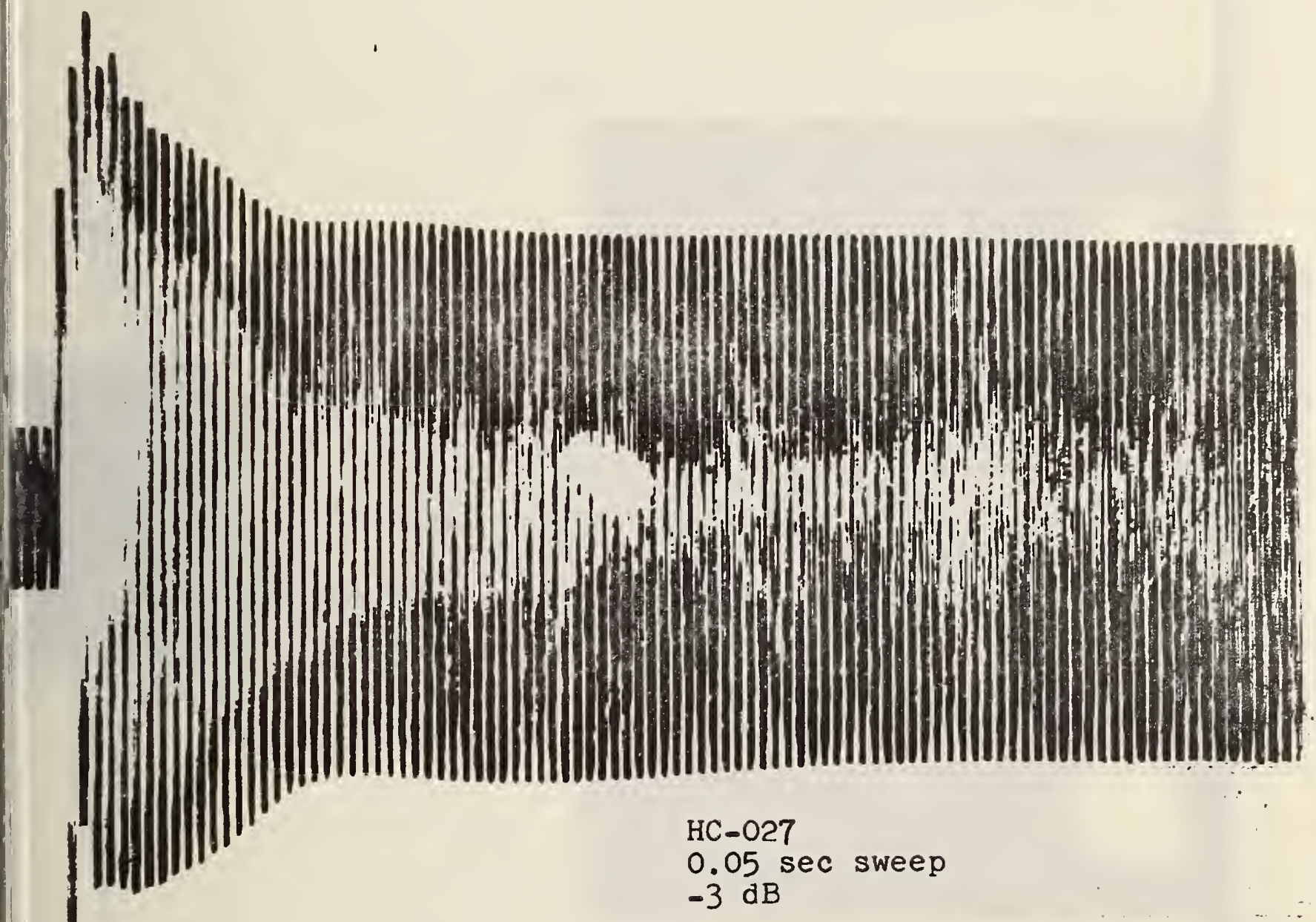




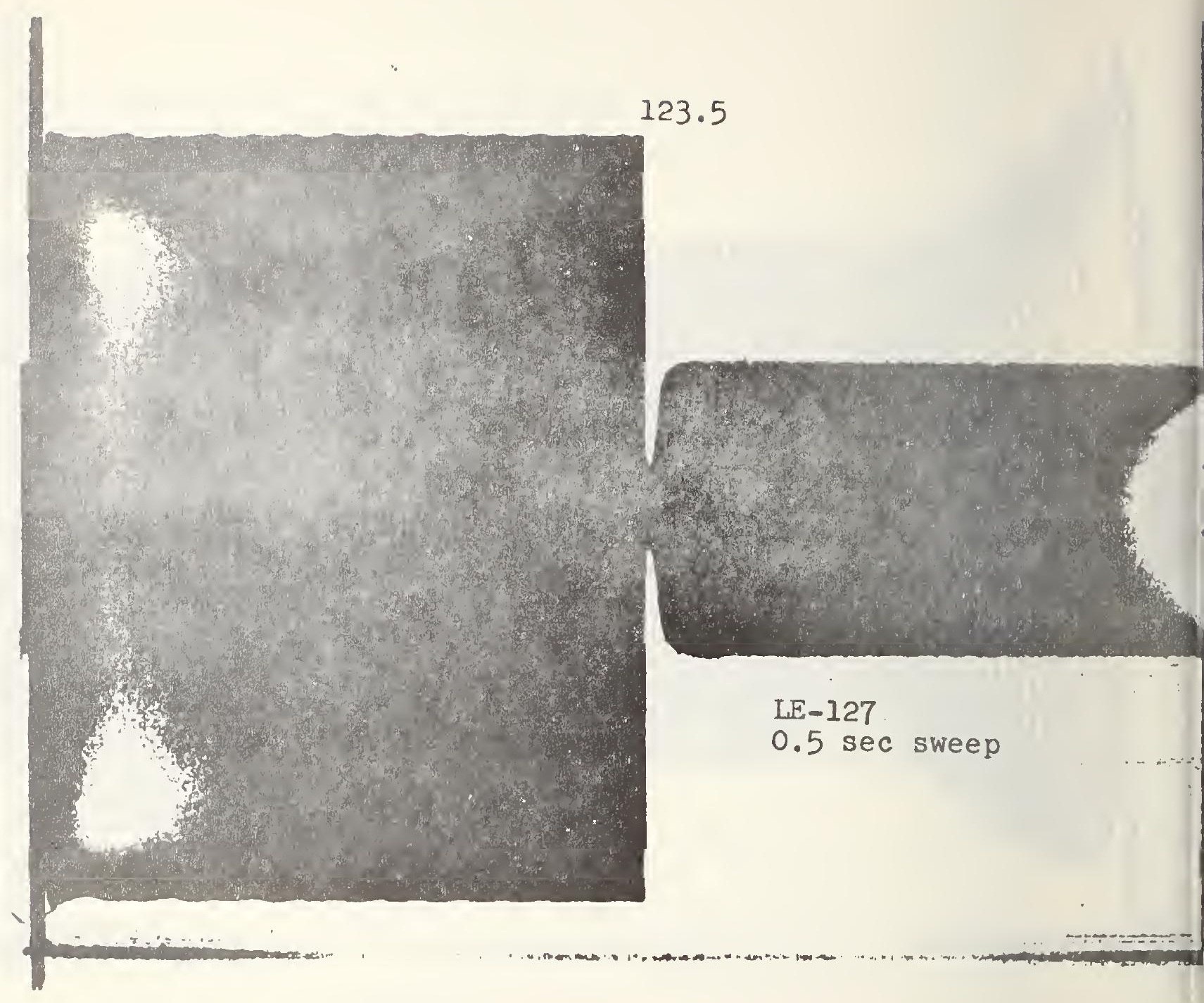




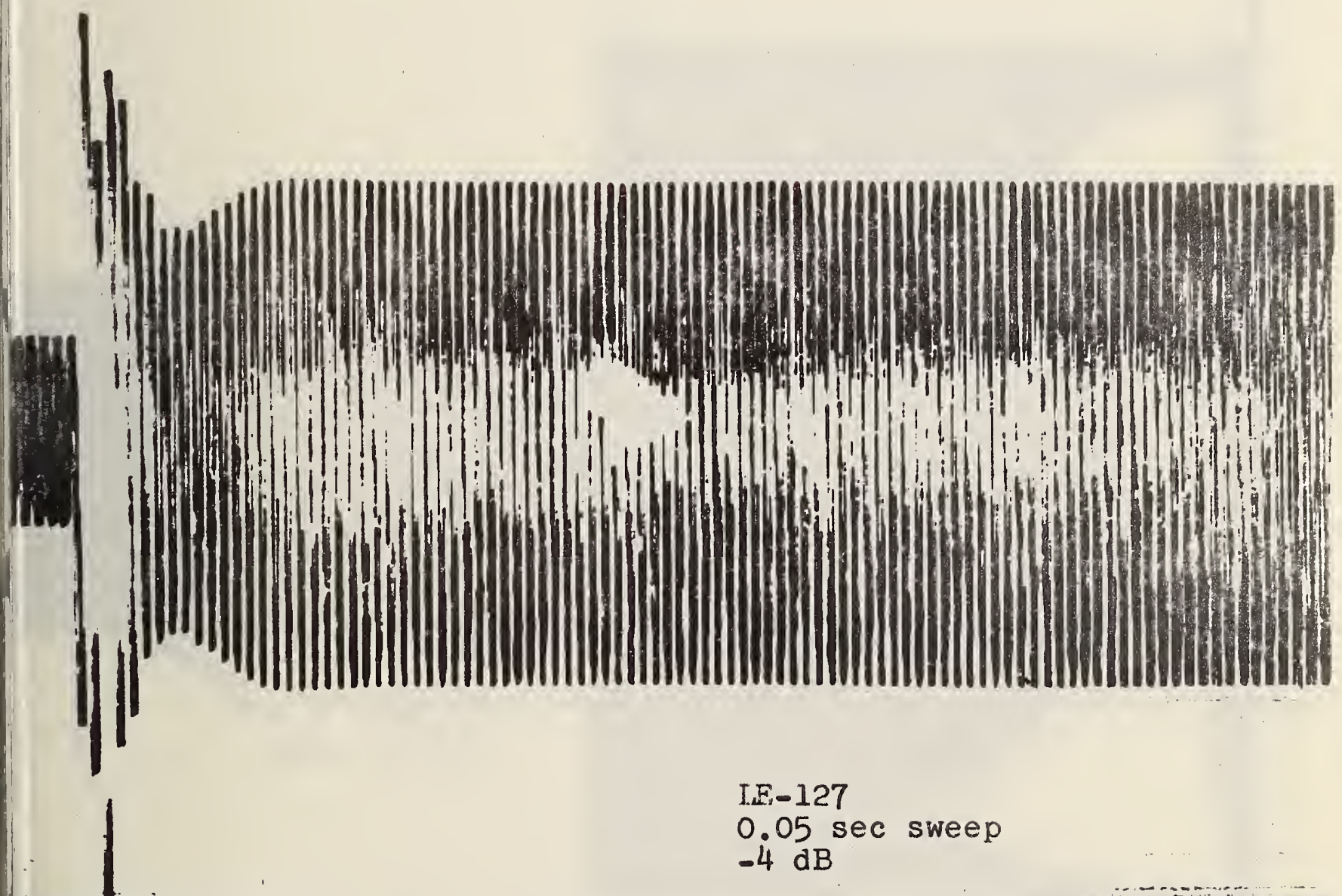




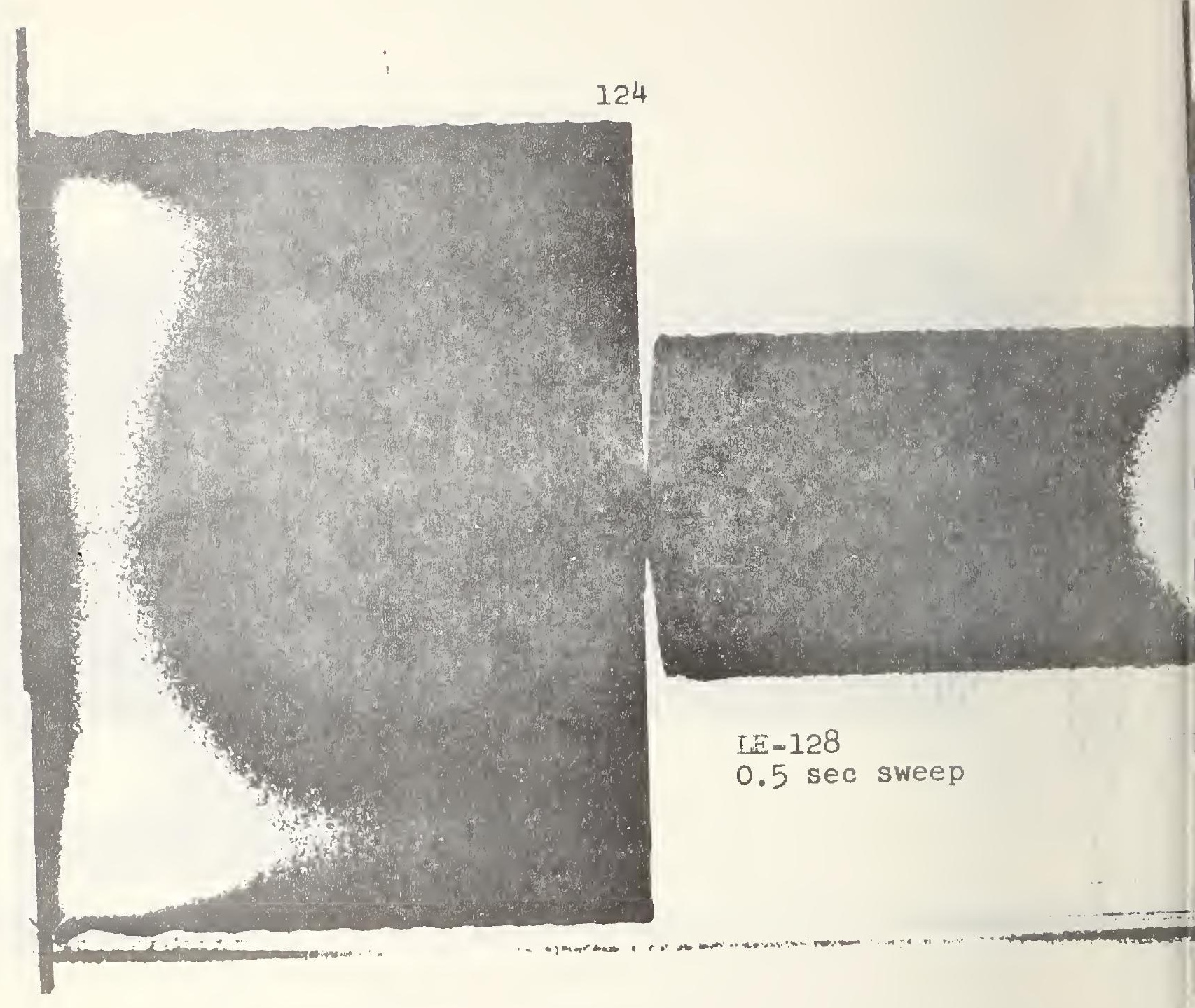




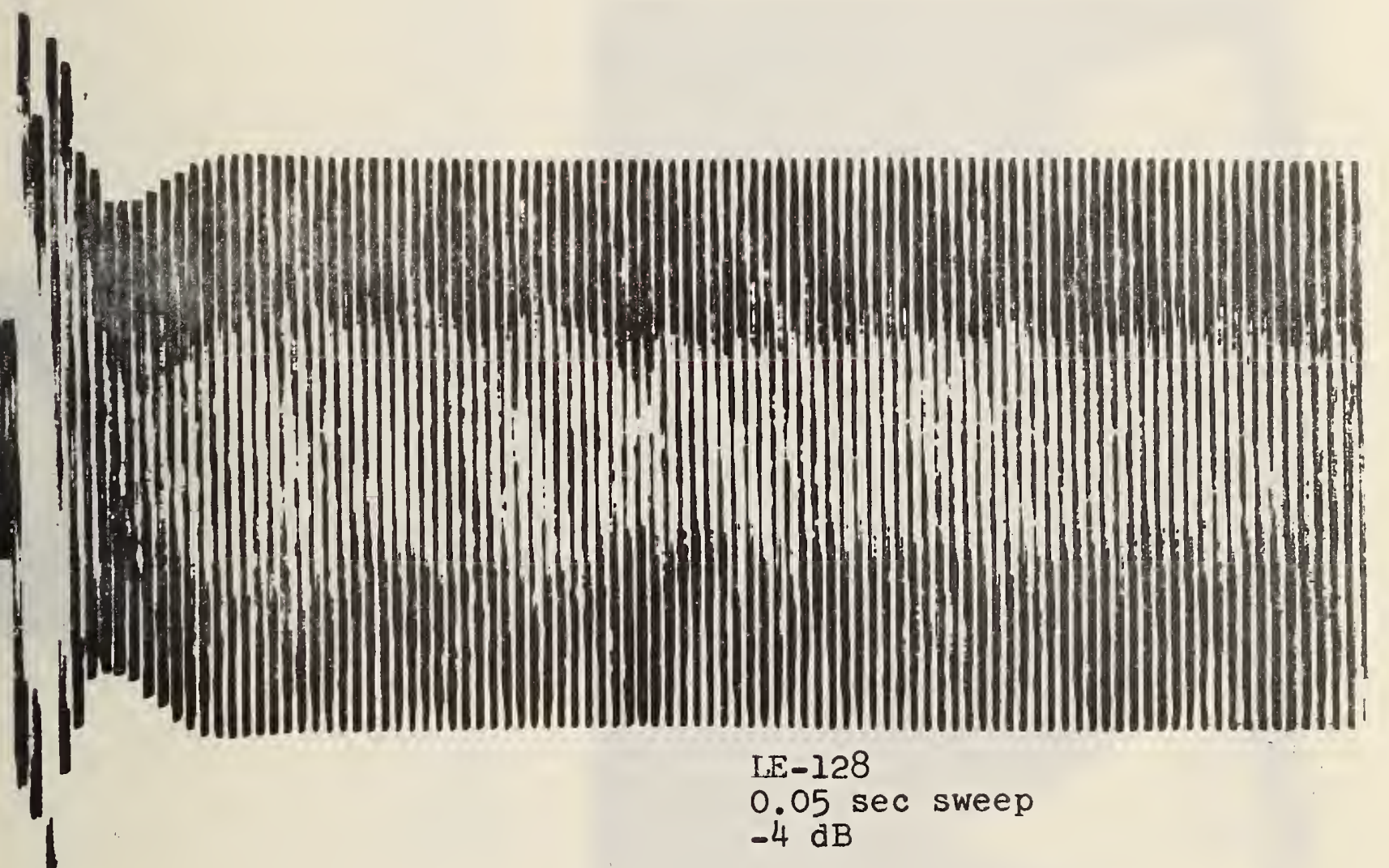

$$
\begin{aligned}
& \text { IE- }-128 \\
& 0.05 \text { sec sweep } \\
& -4 \mathrm{~dB}
\end{aligned}
$$




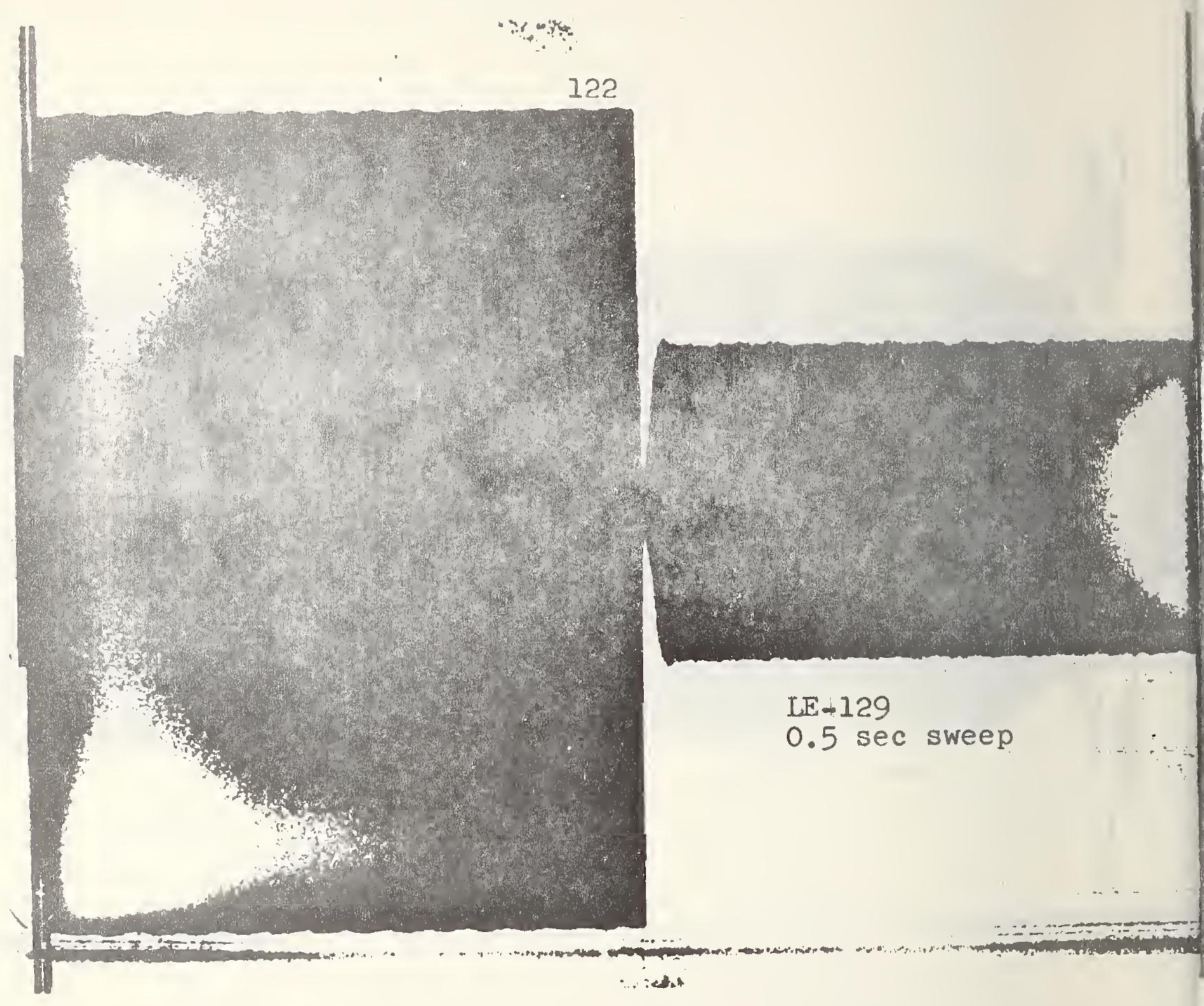



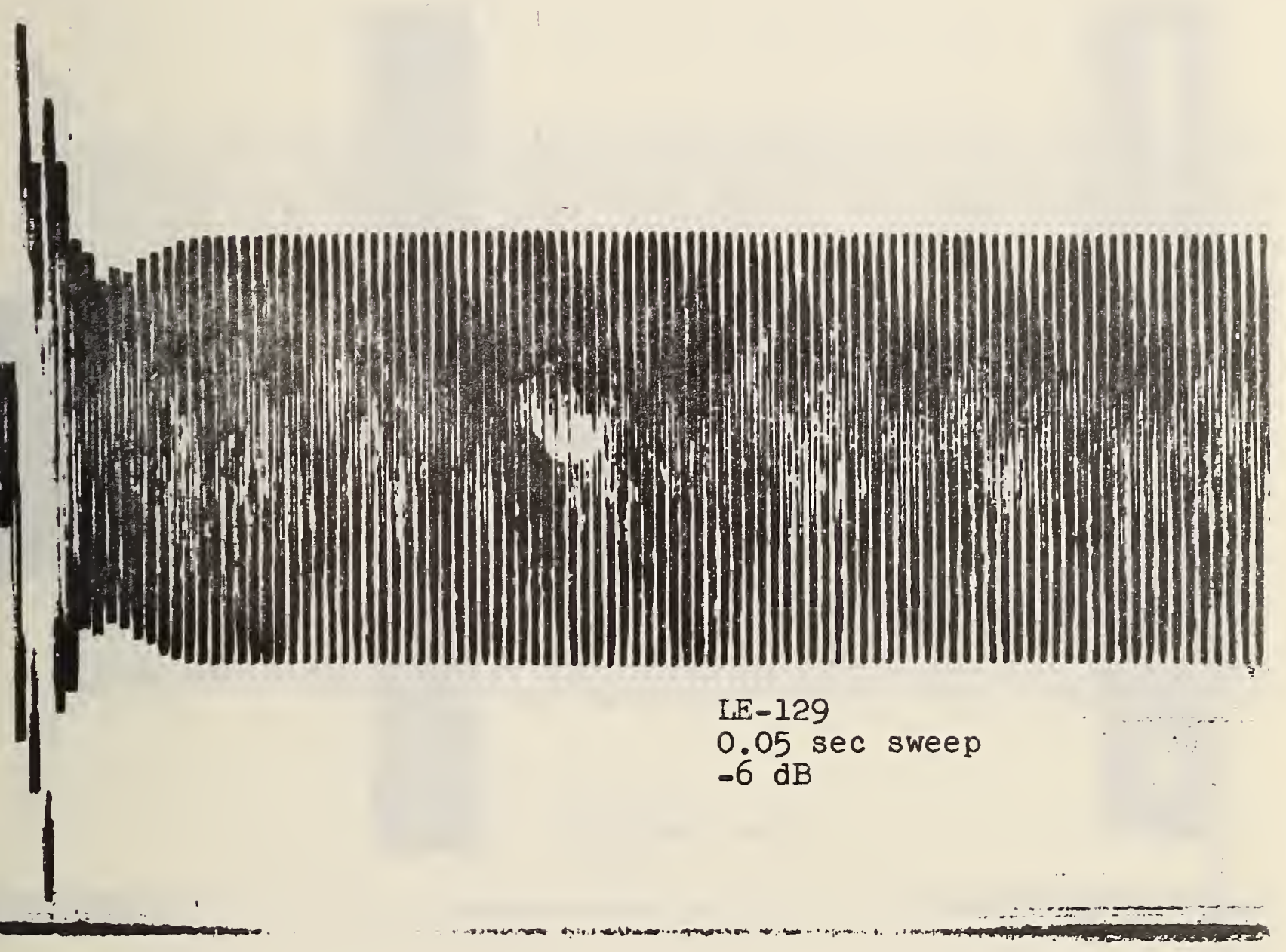


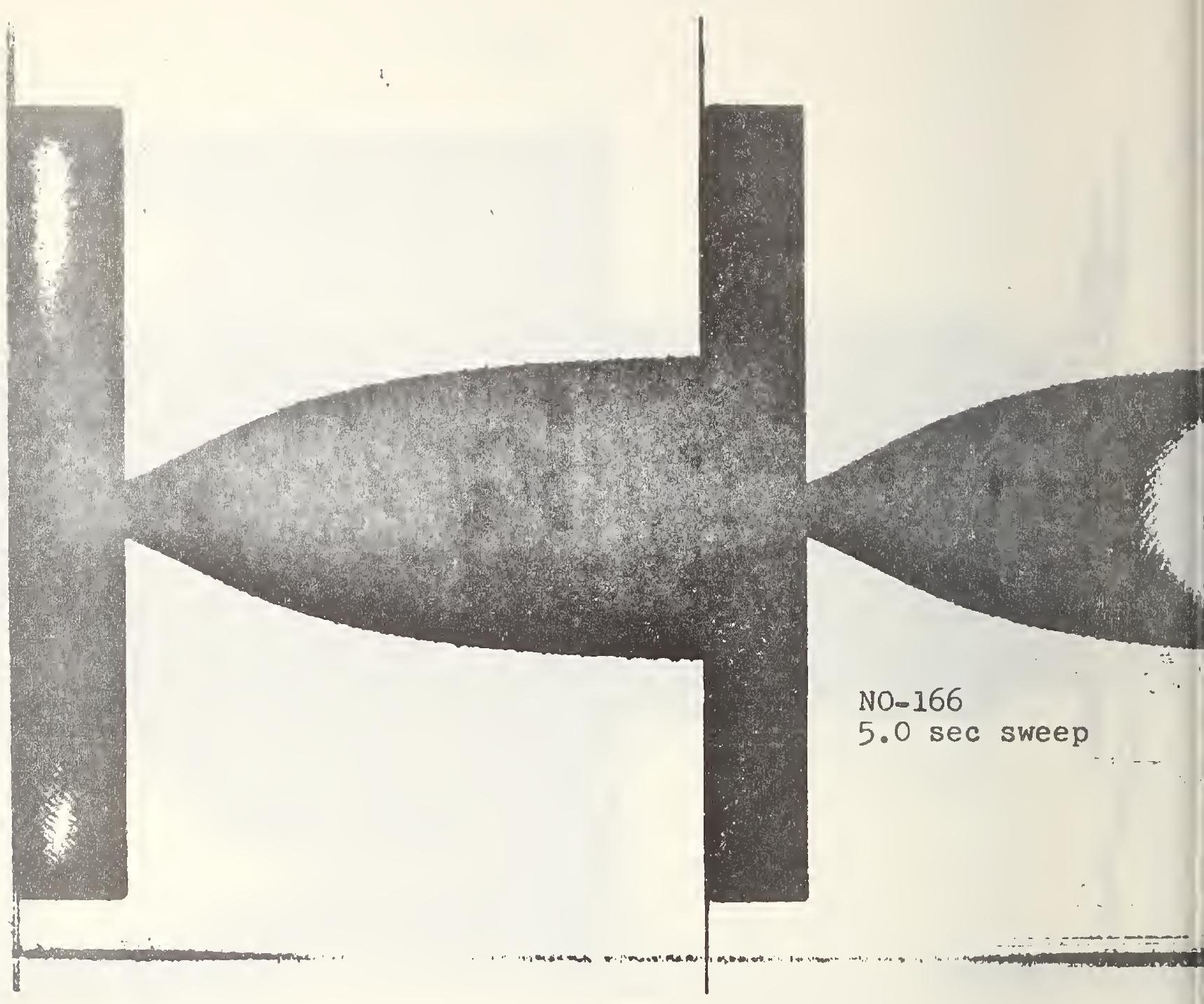



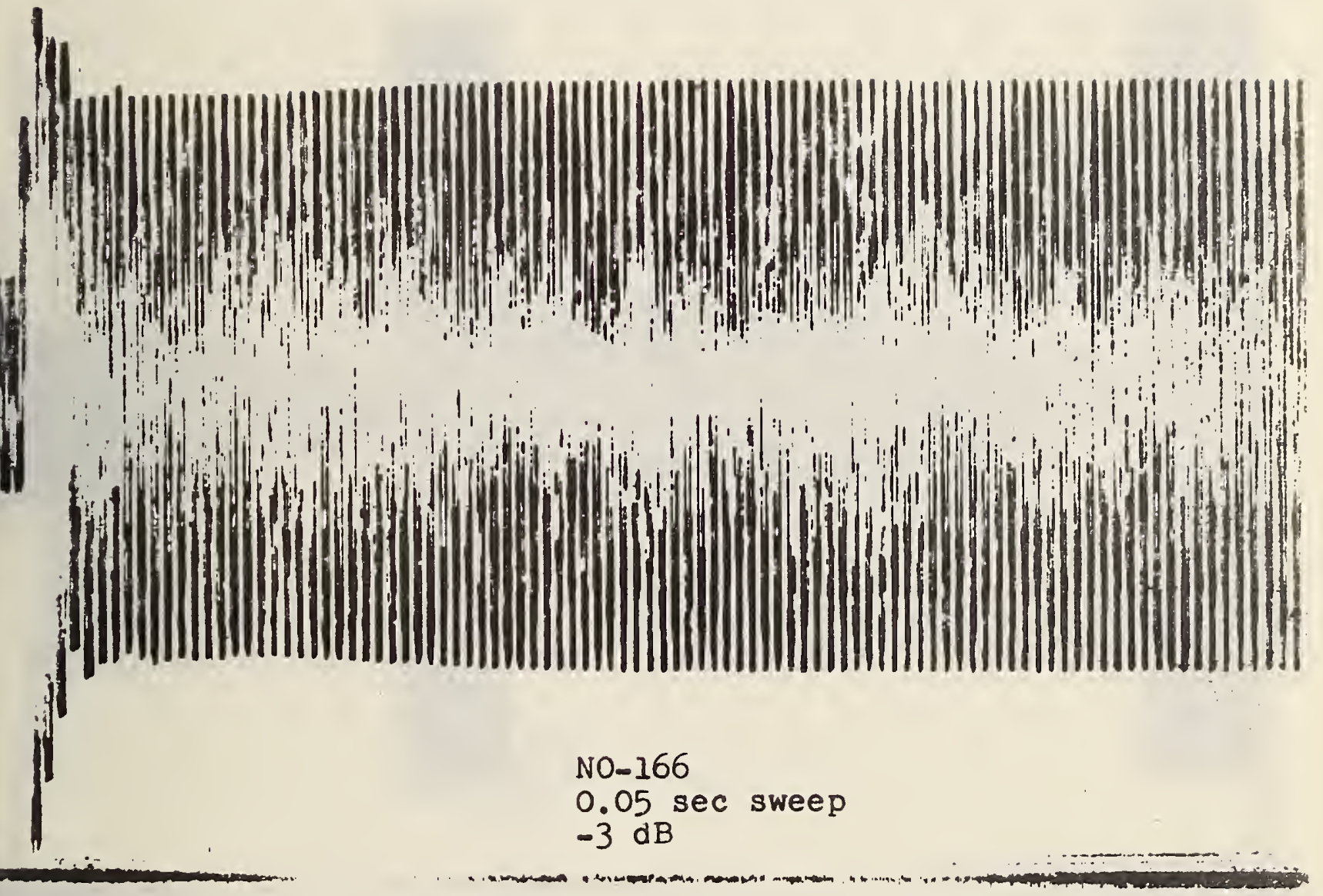


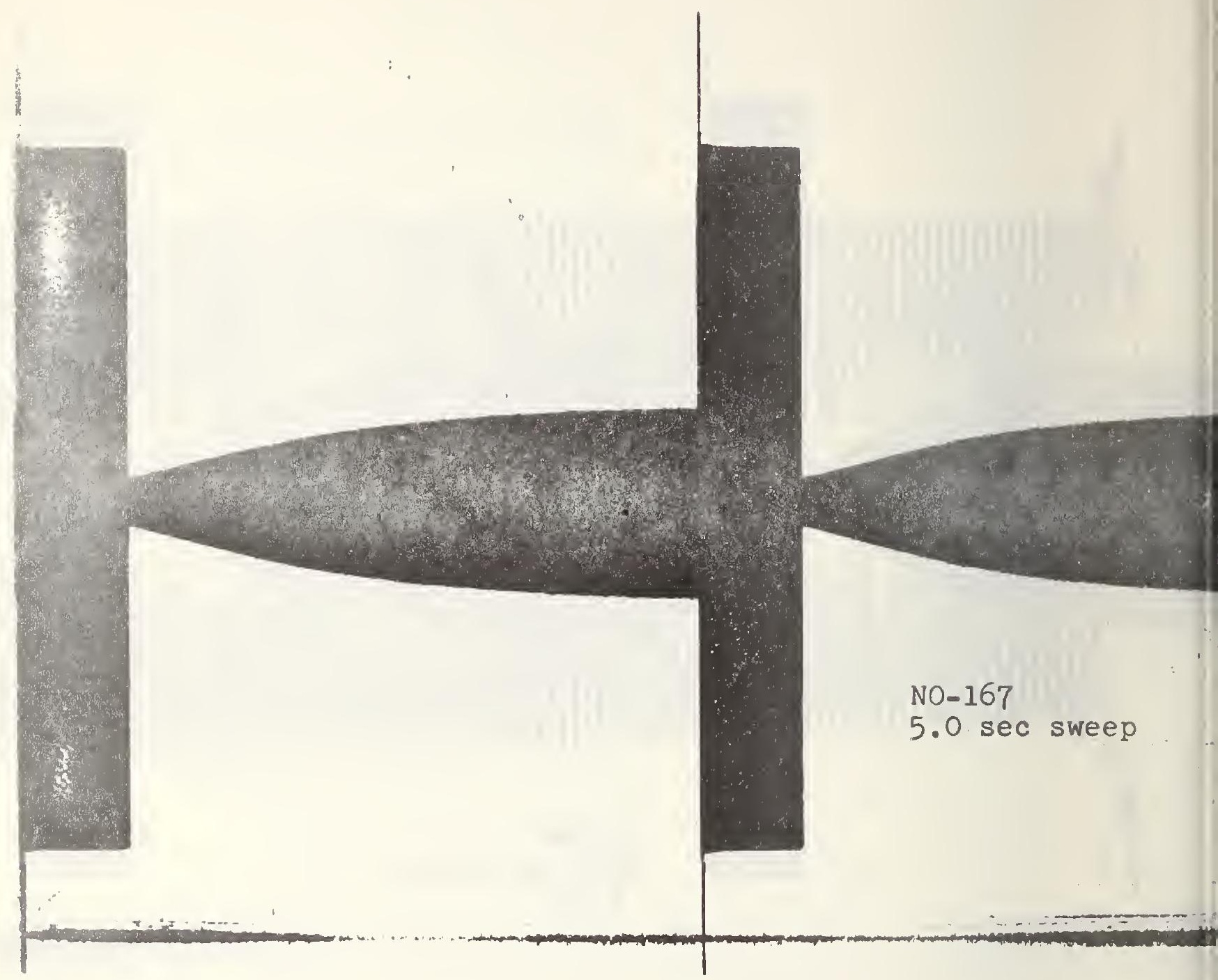




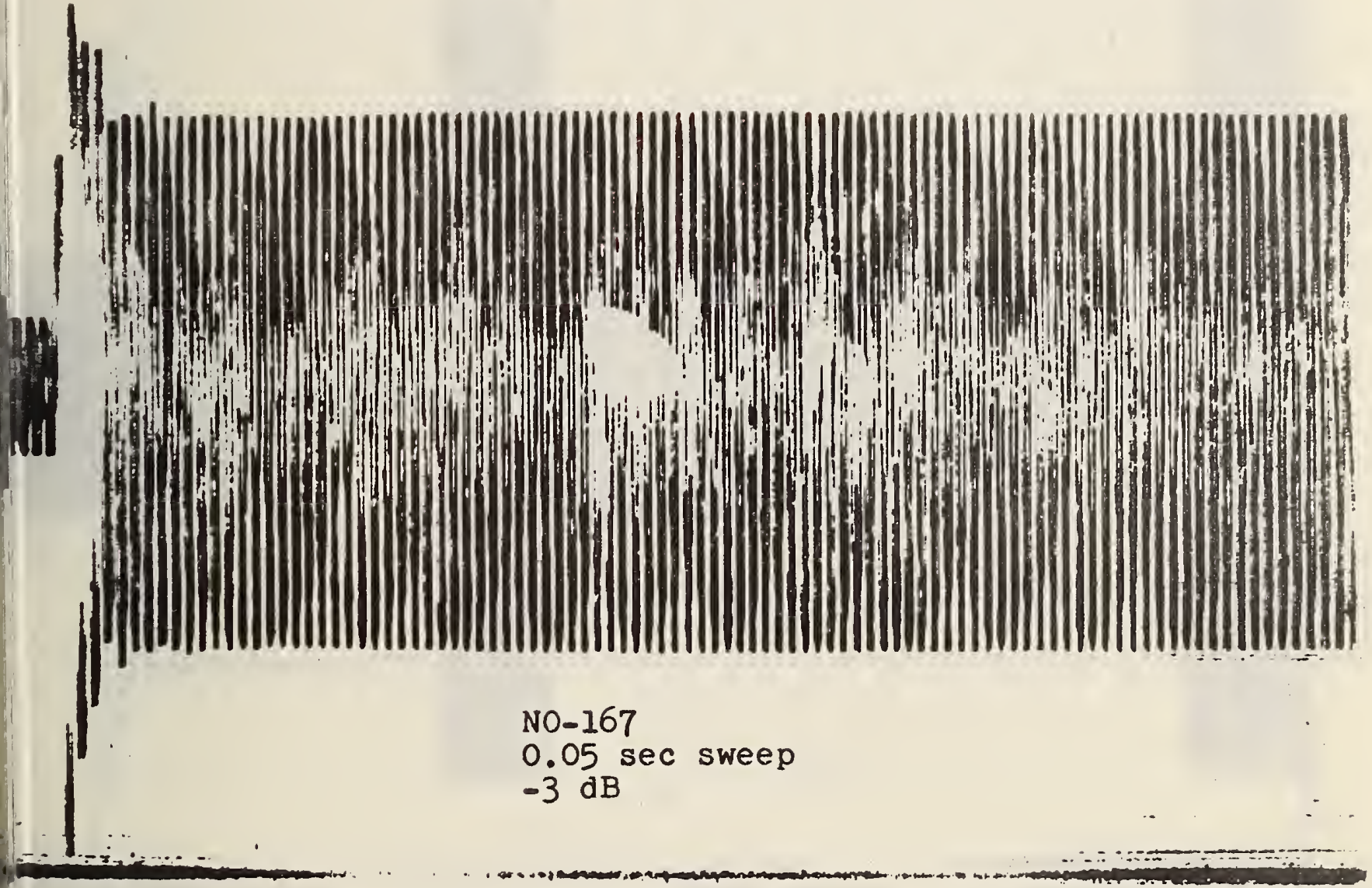




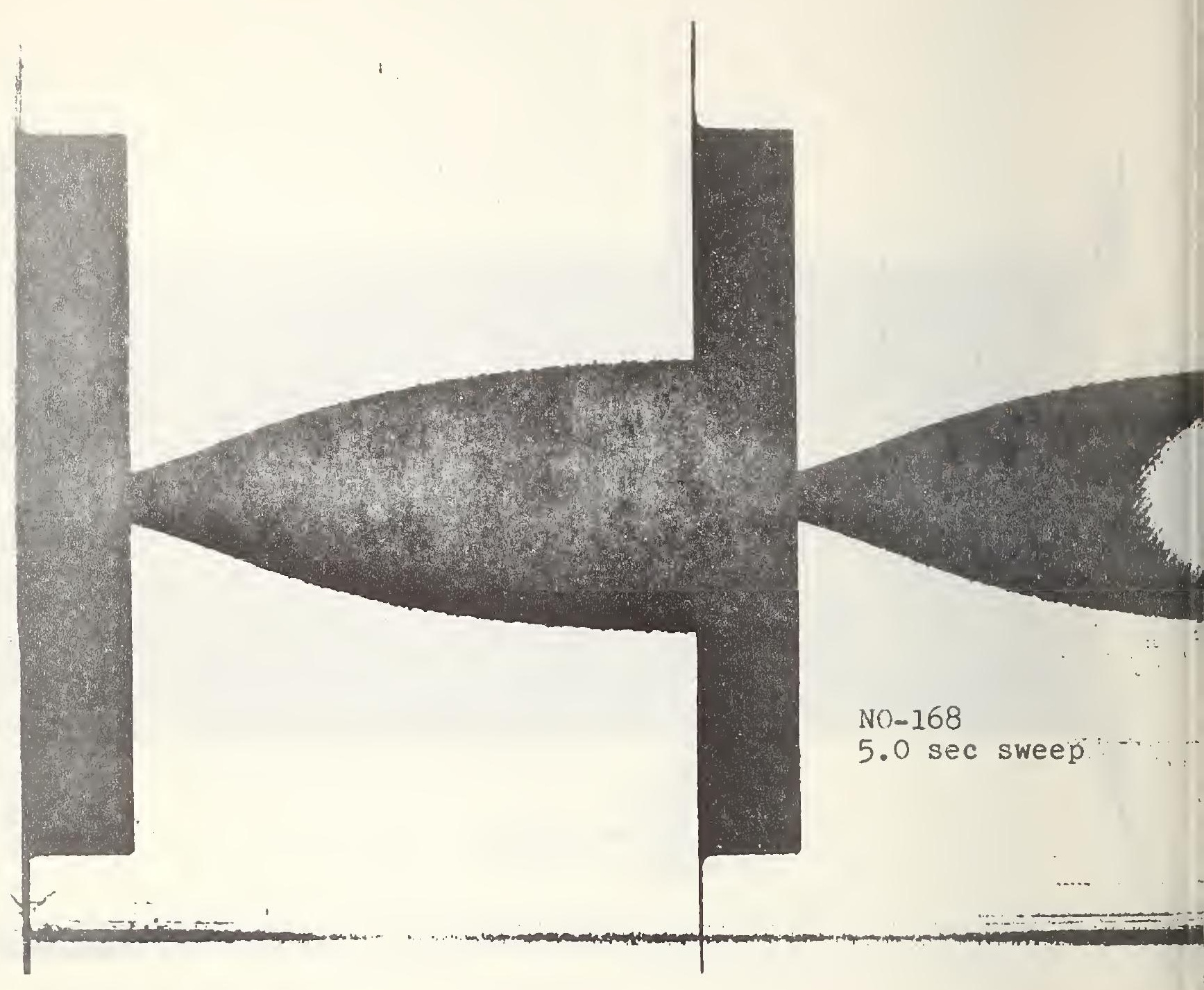




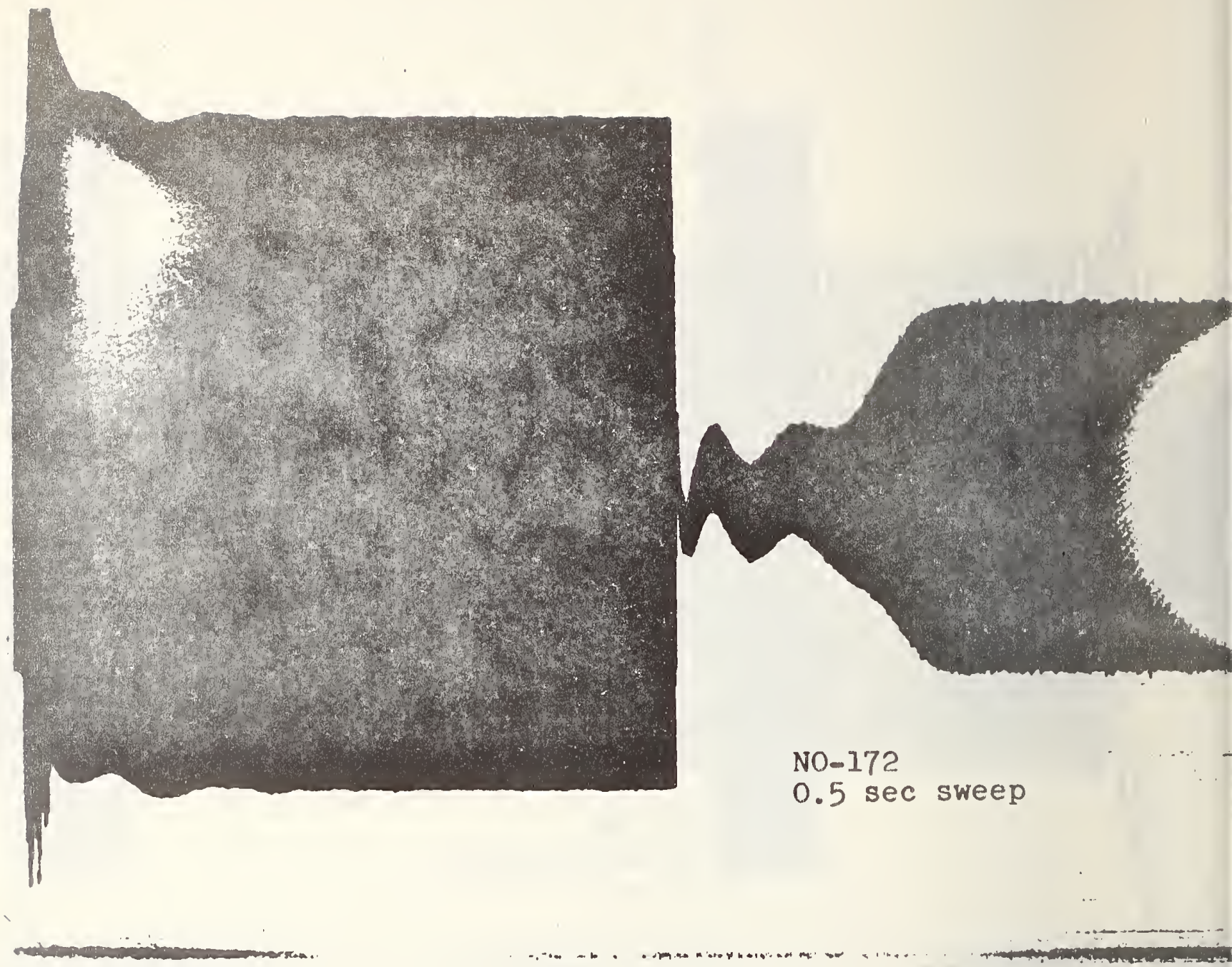




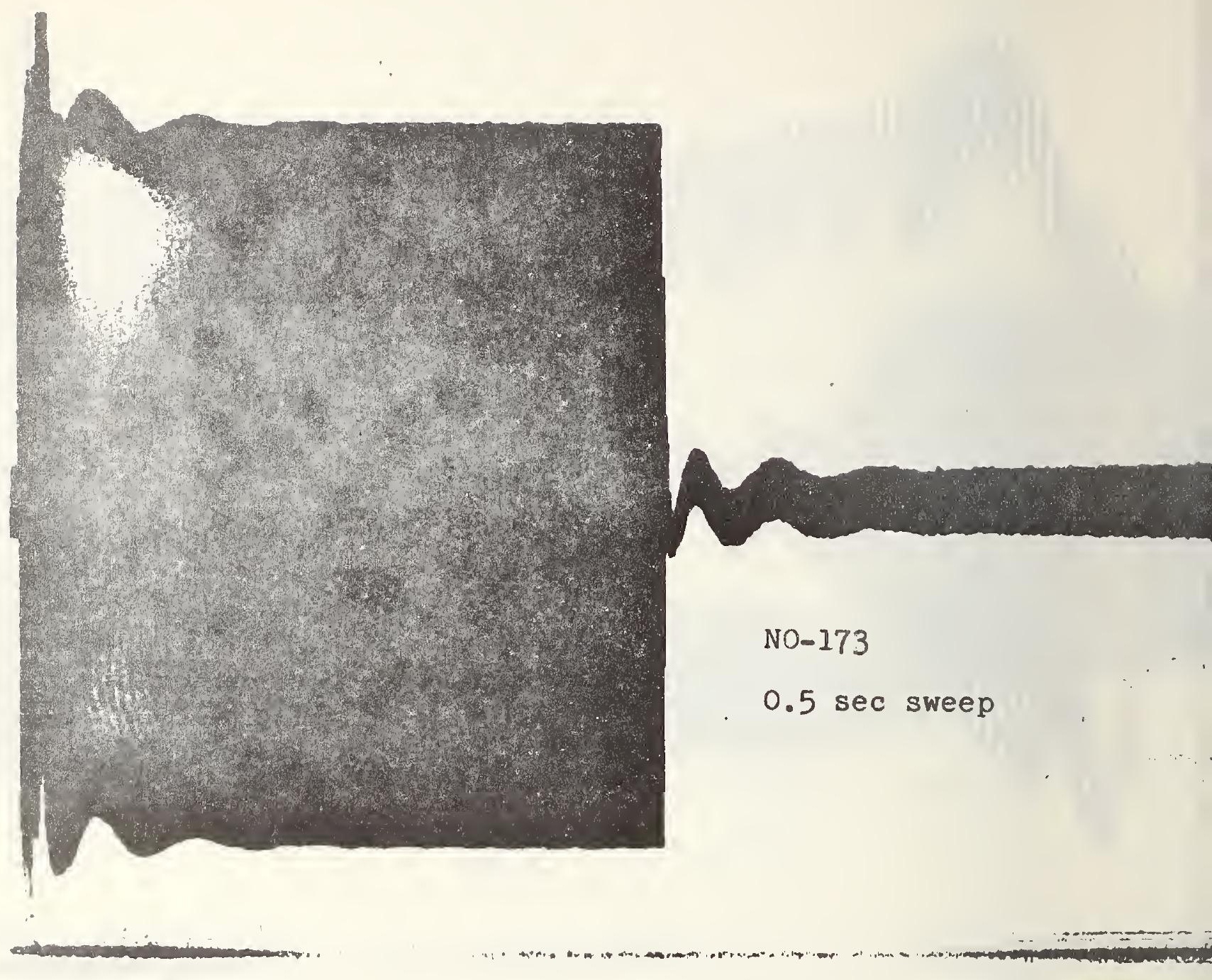




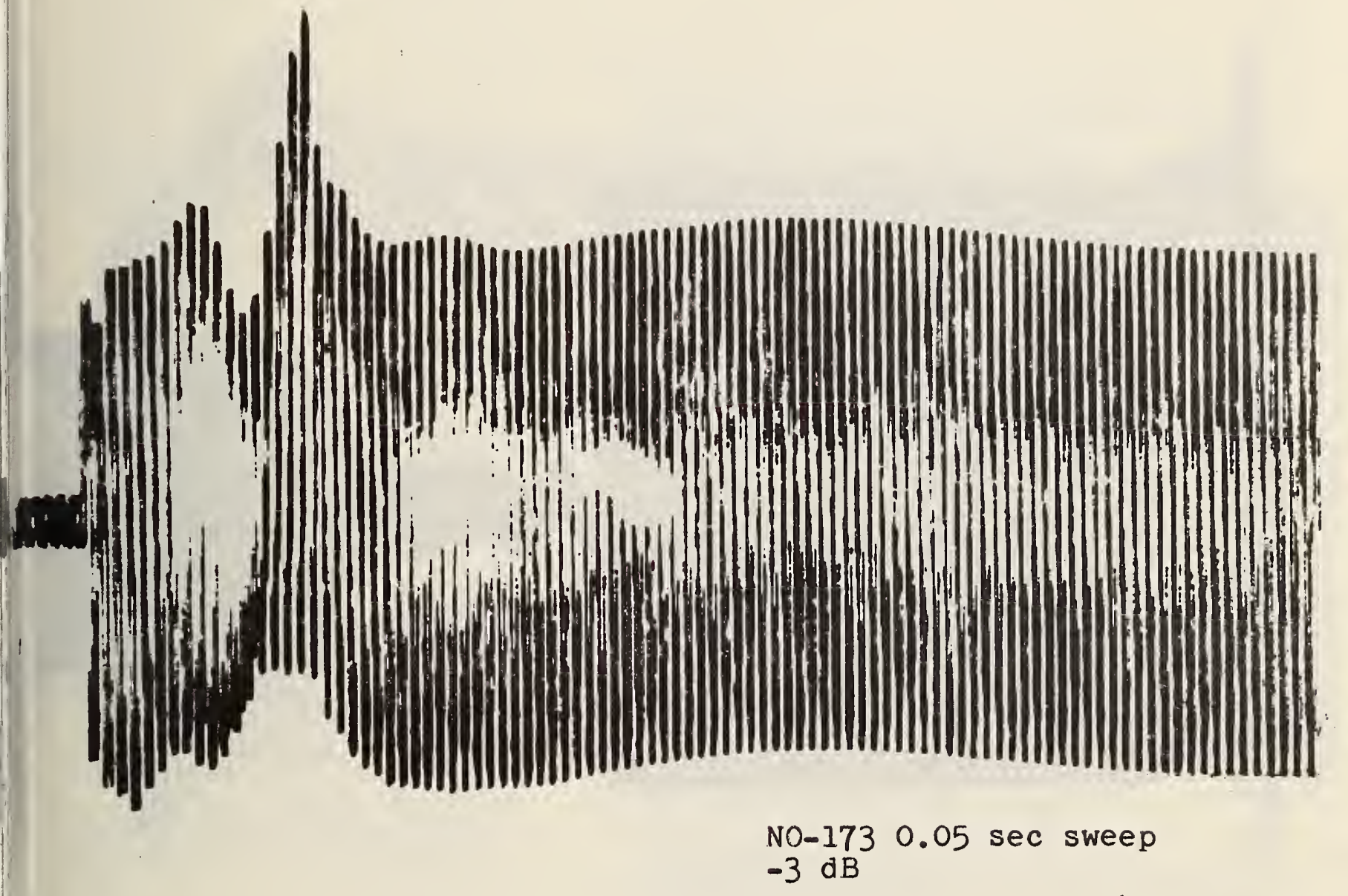




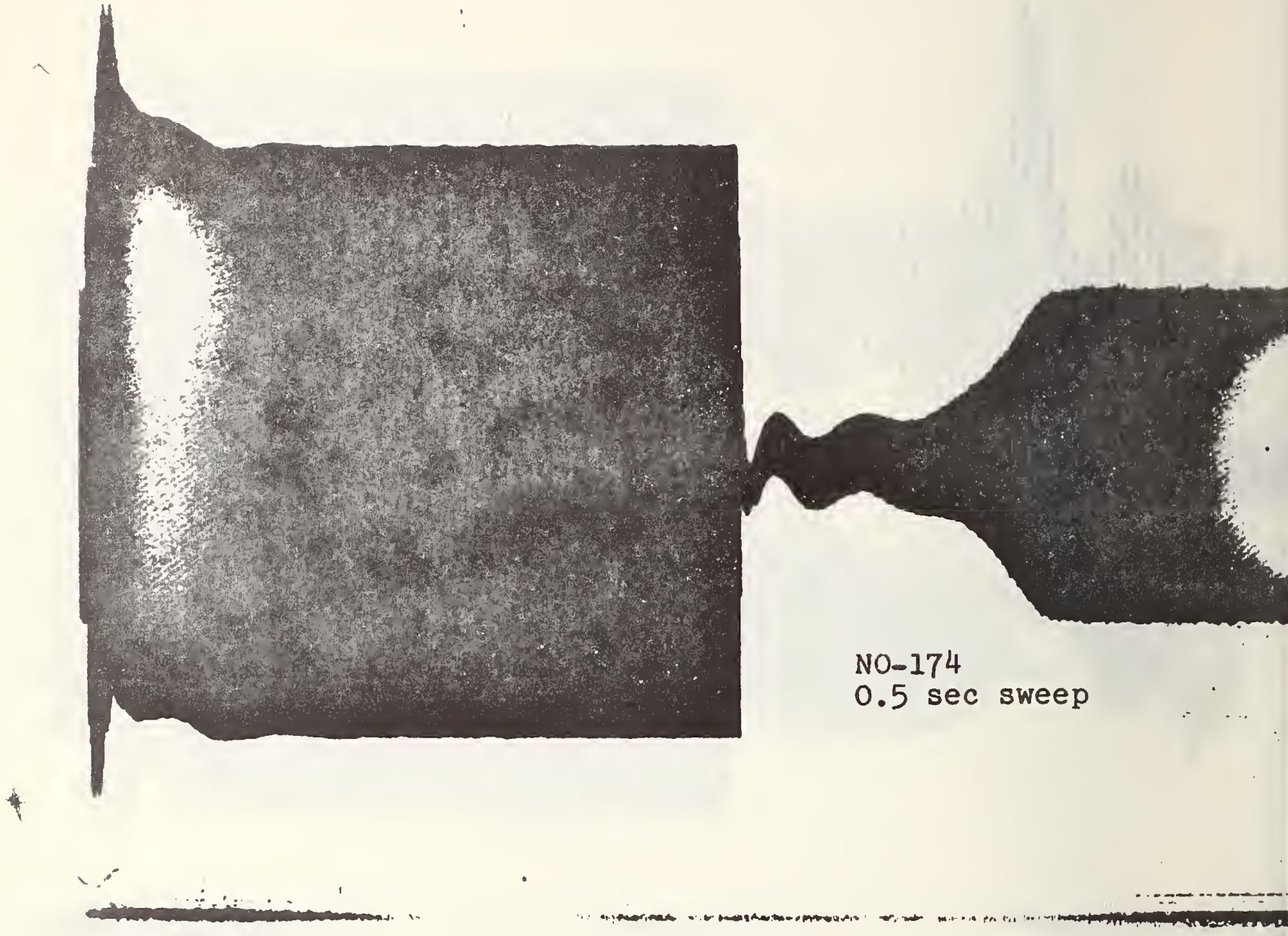




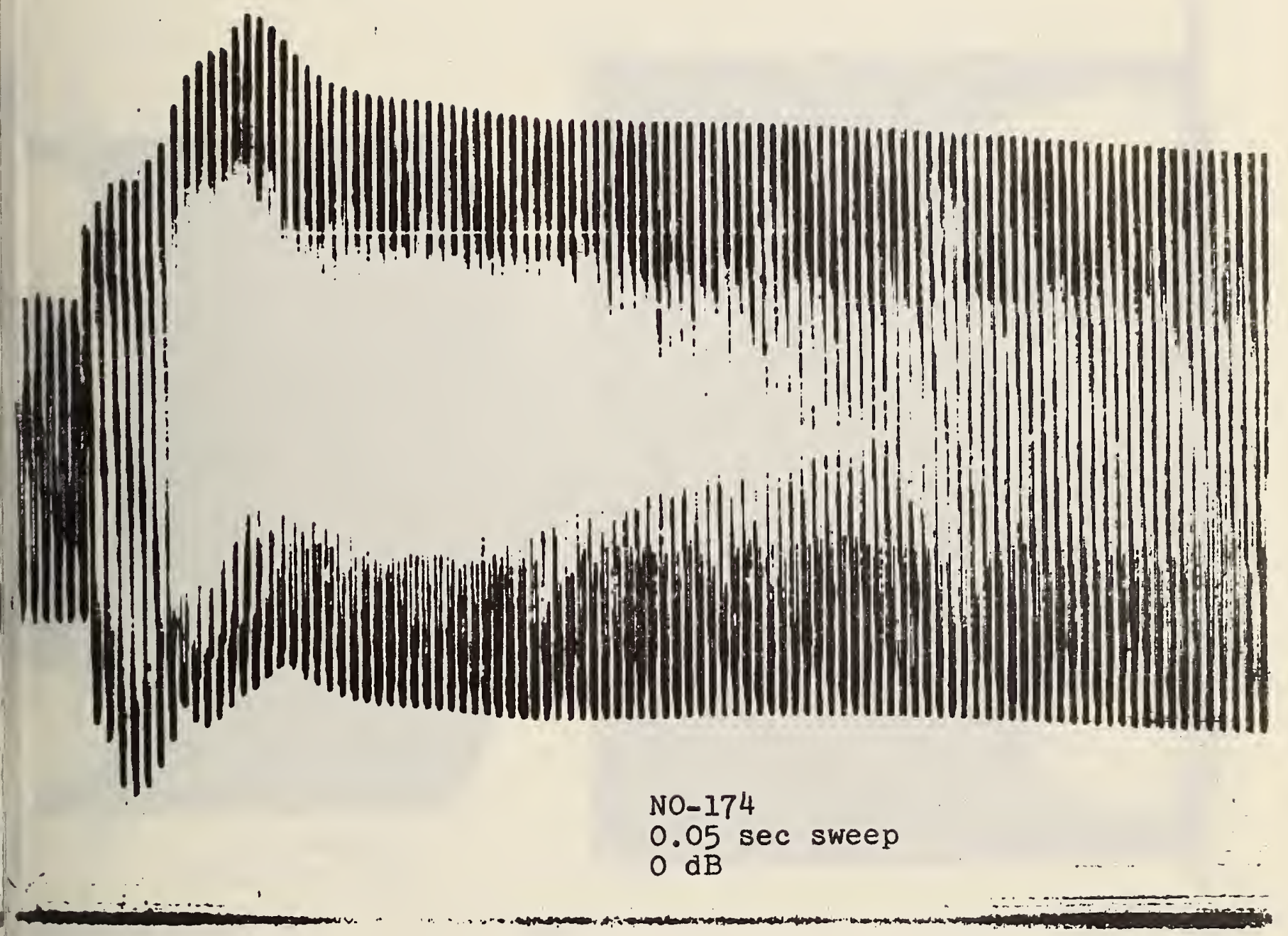




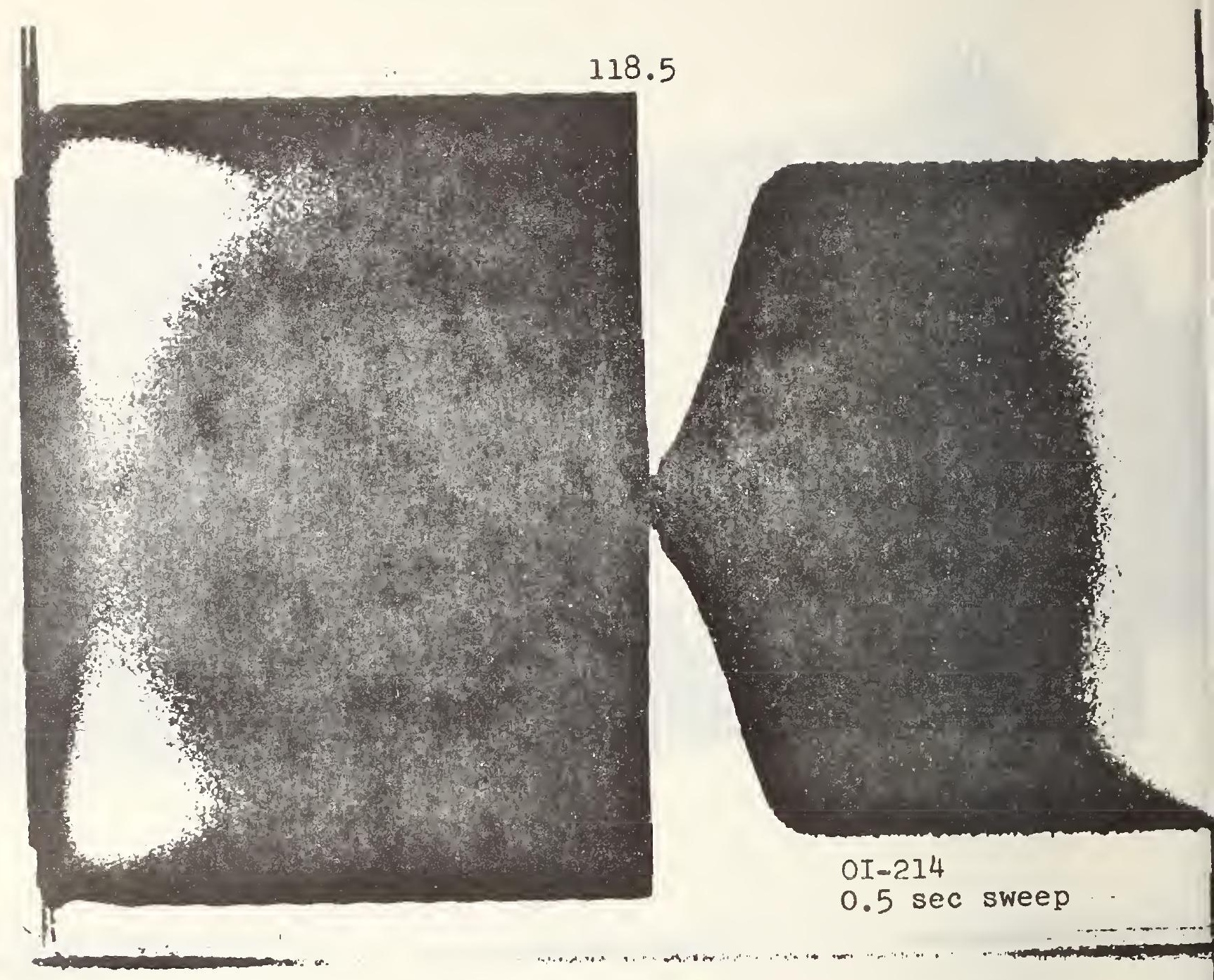




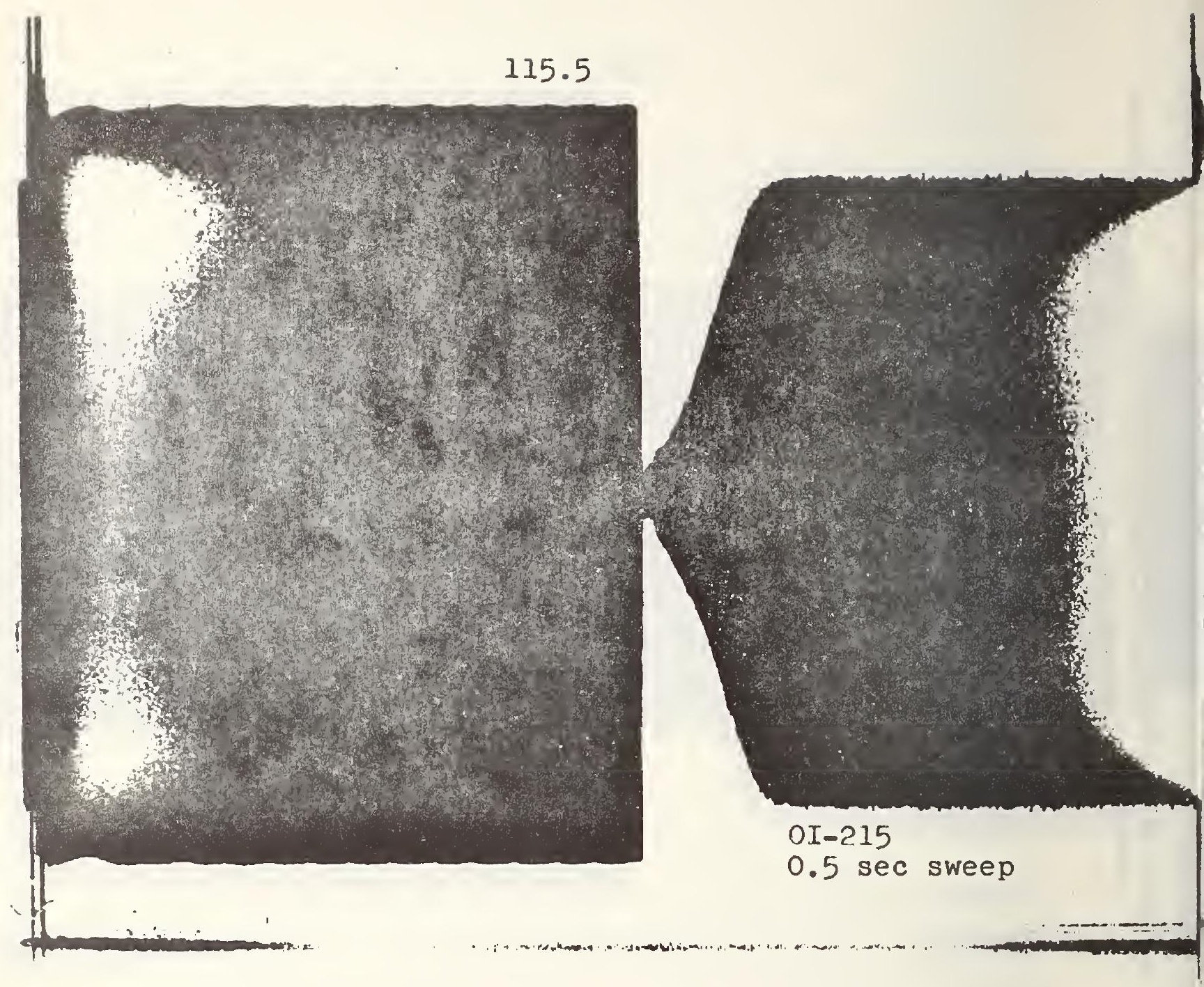


1

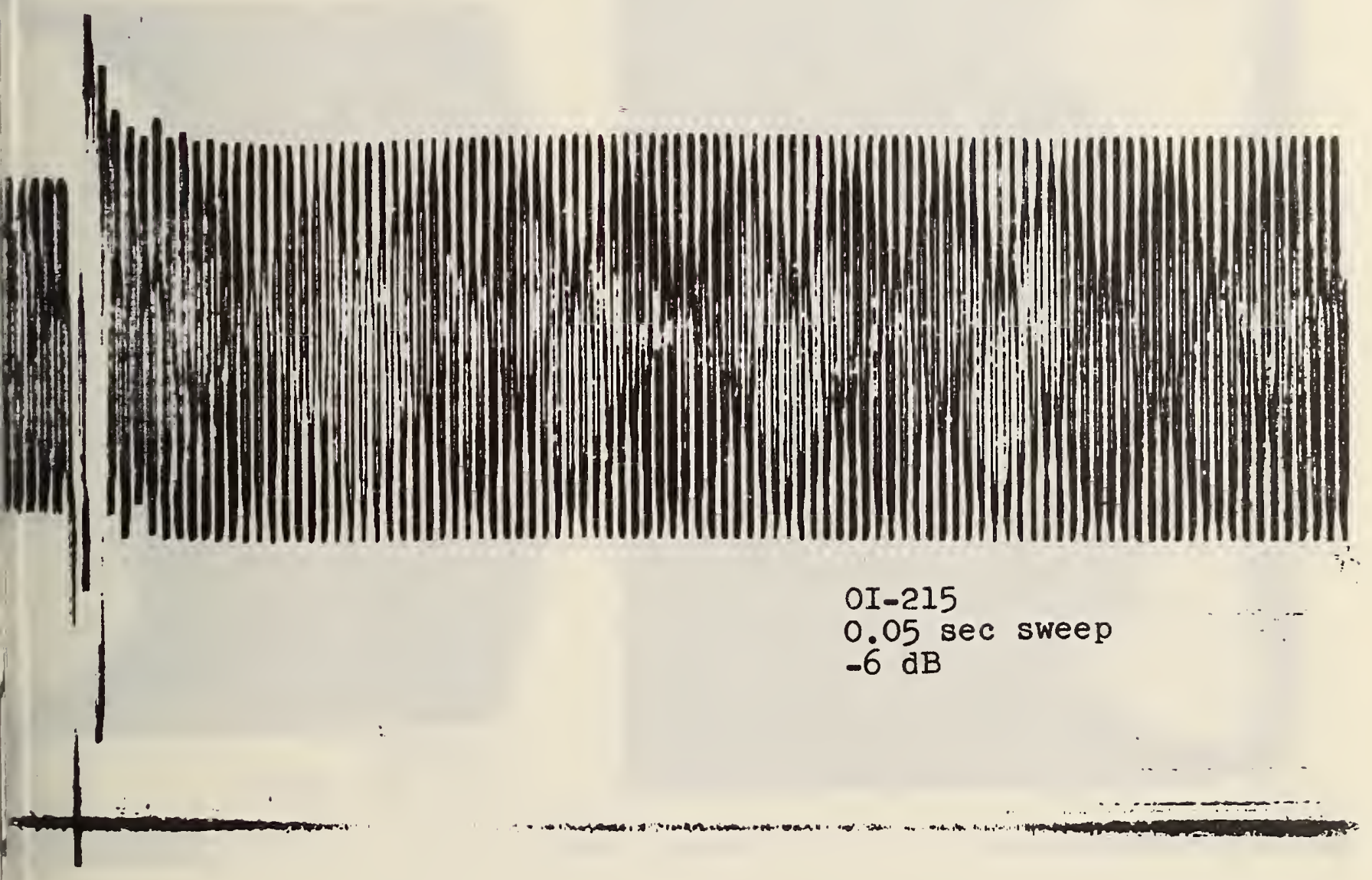




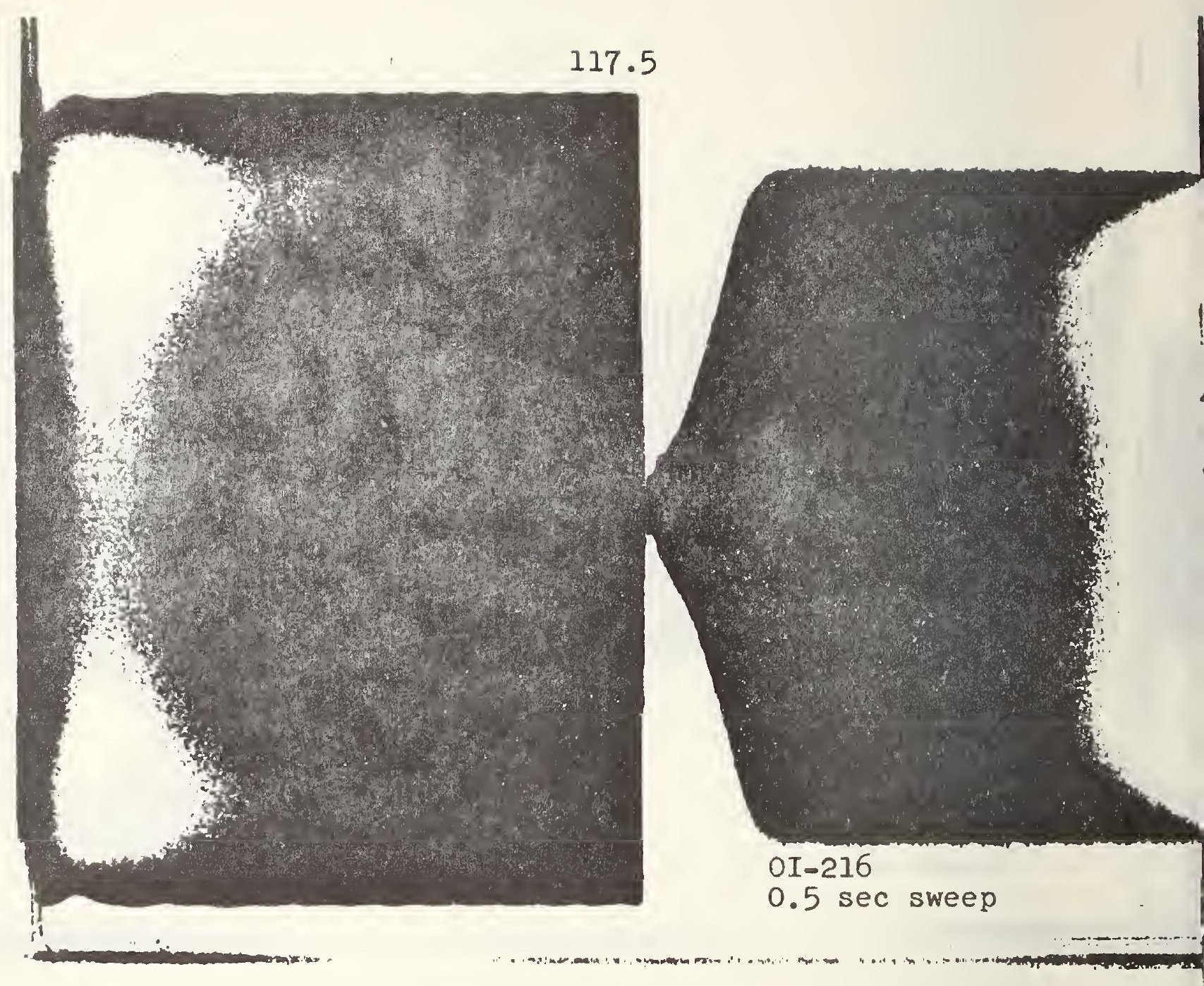




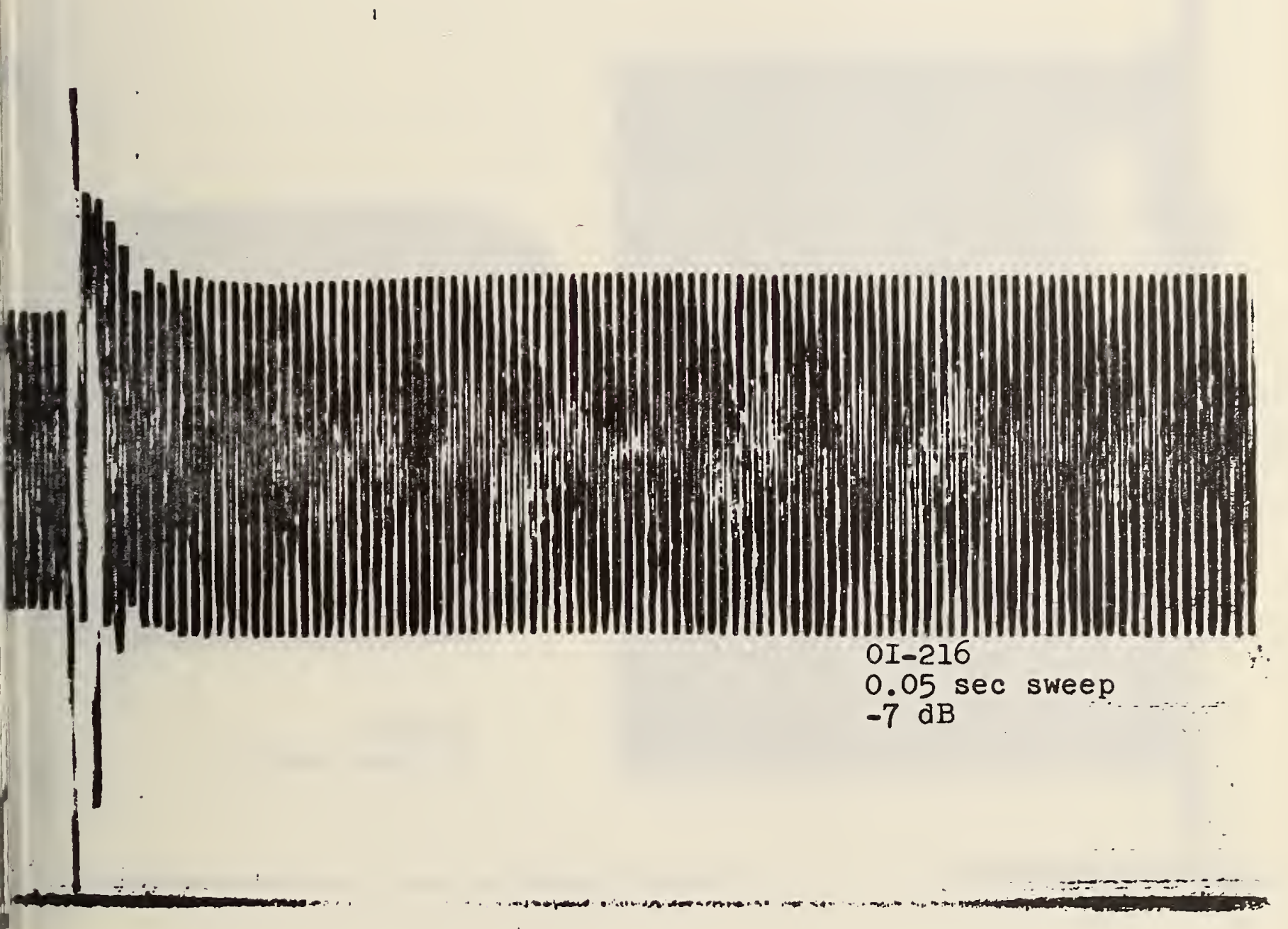




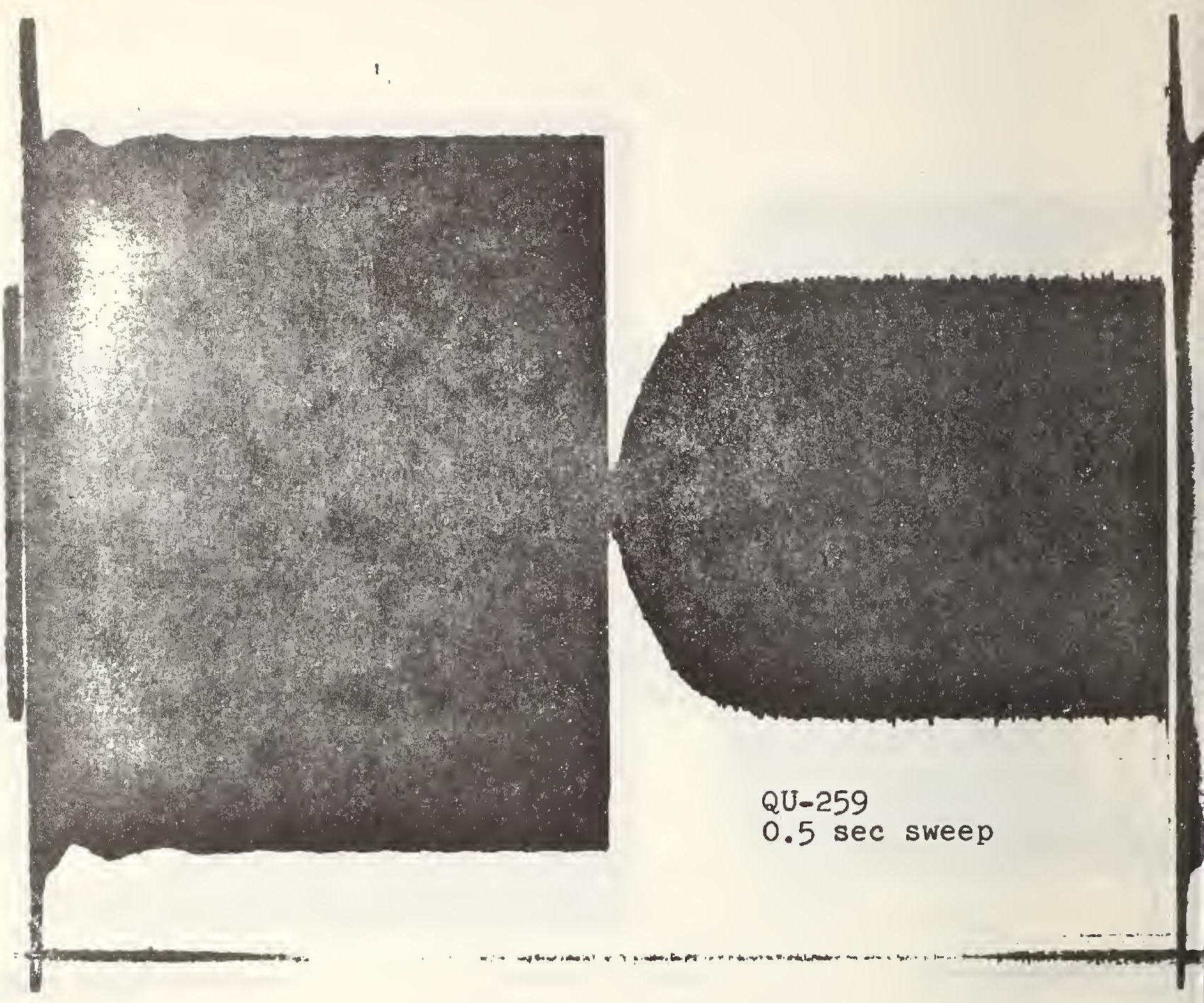




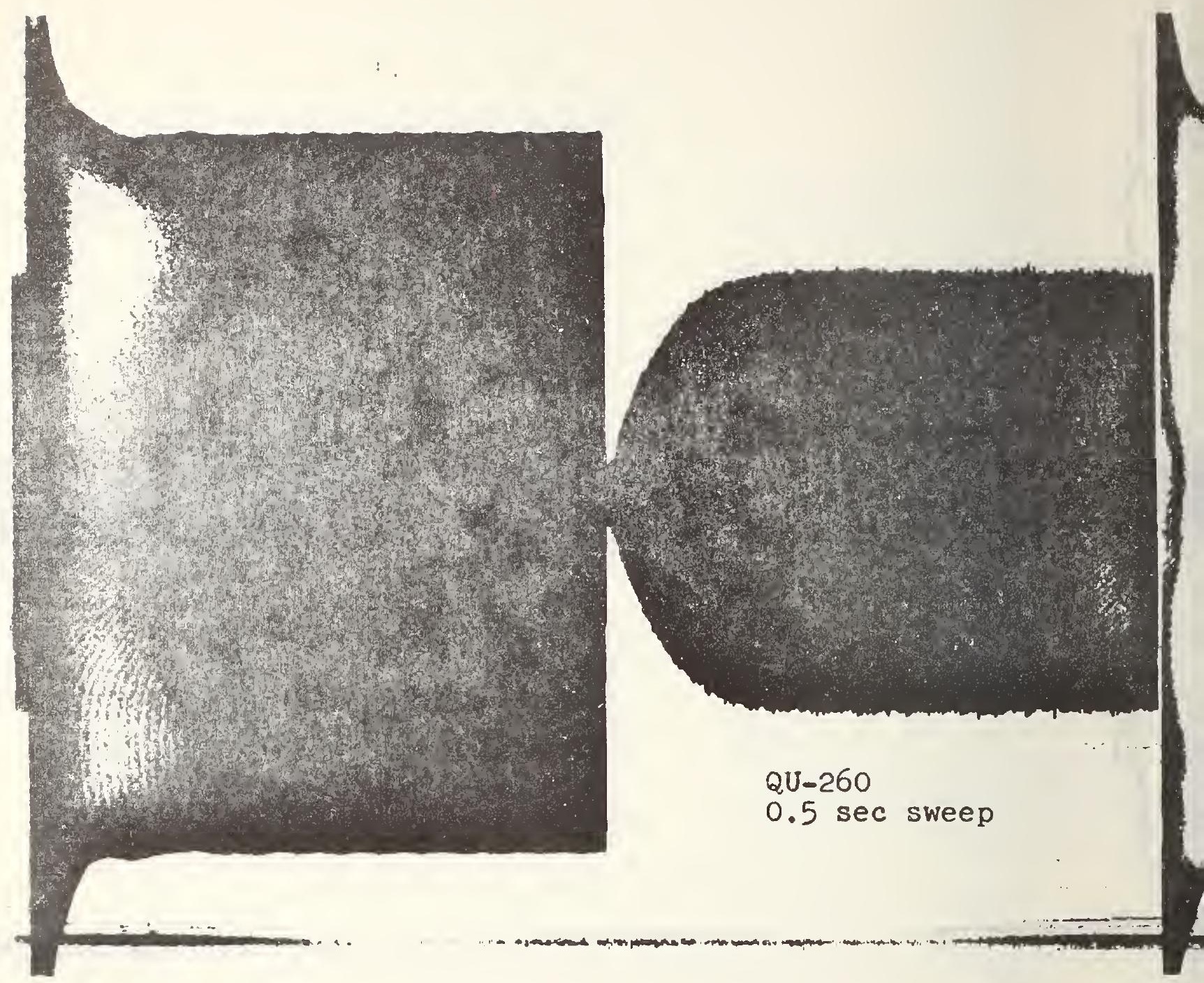




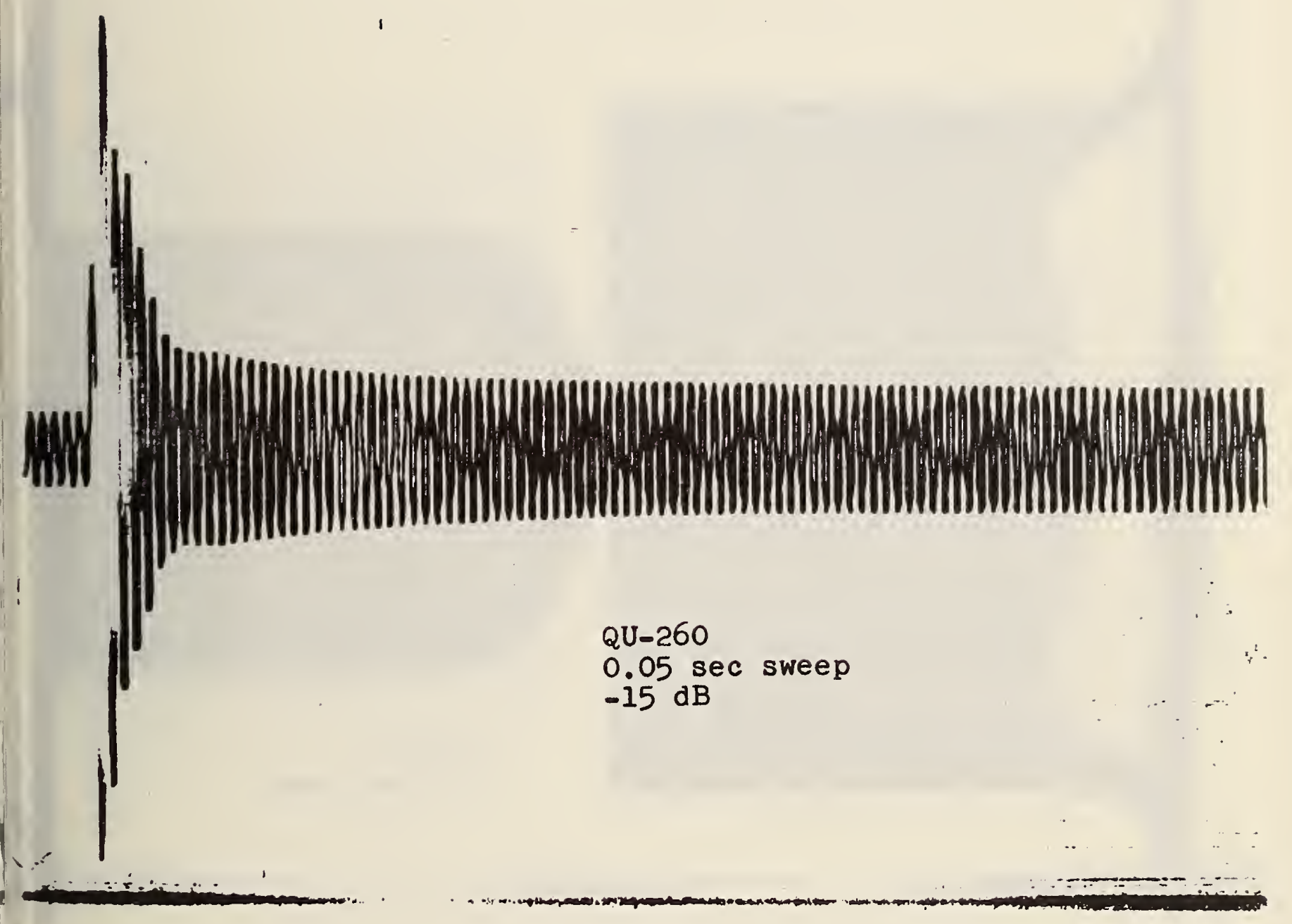




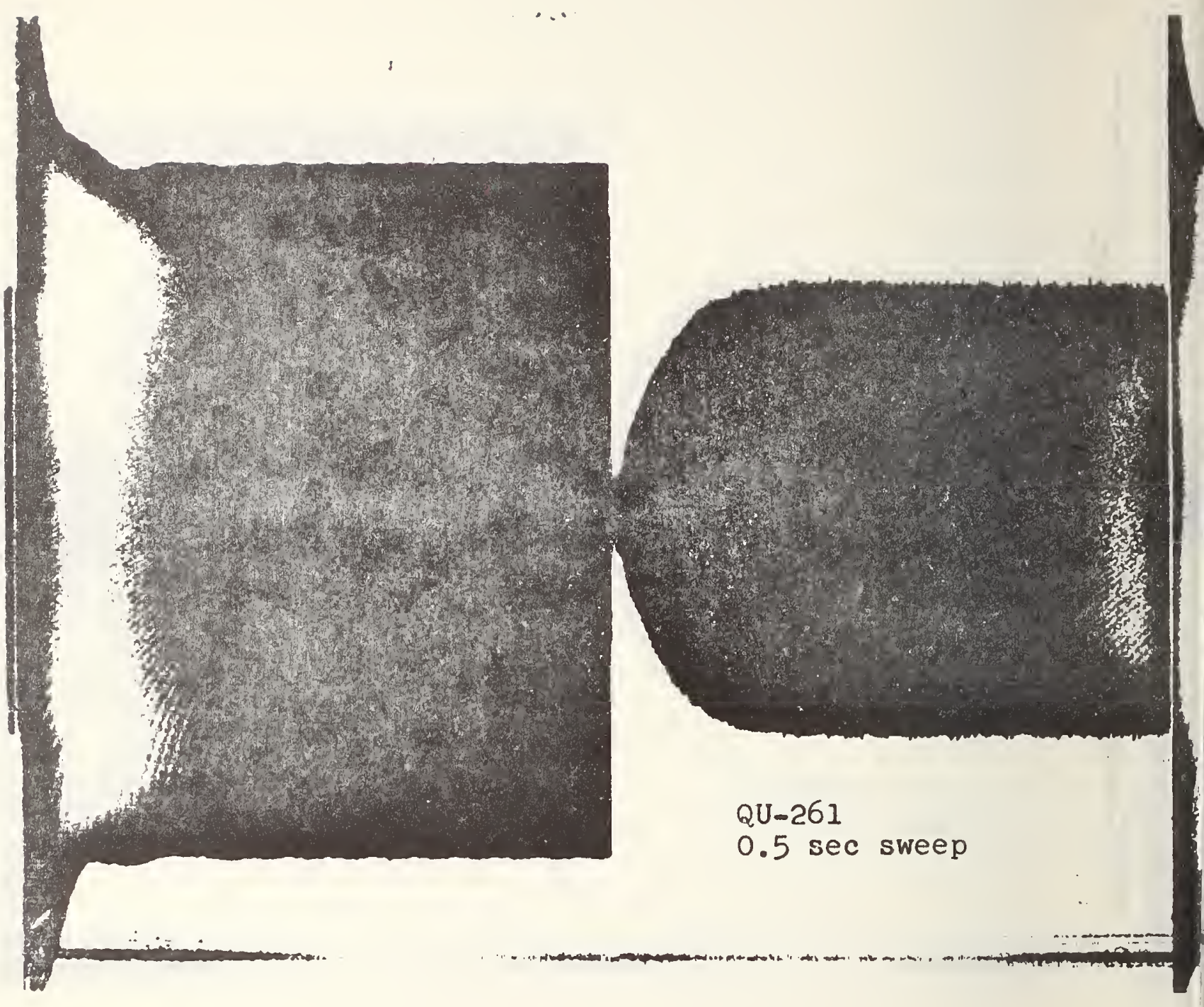




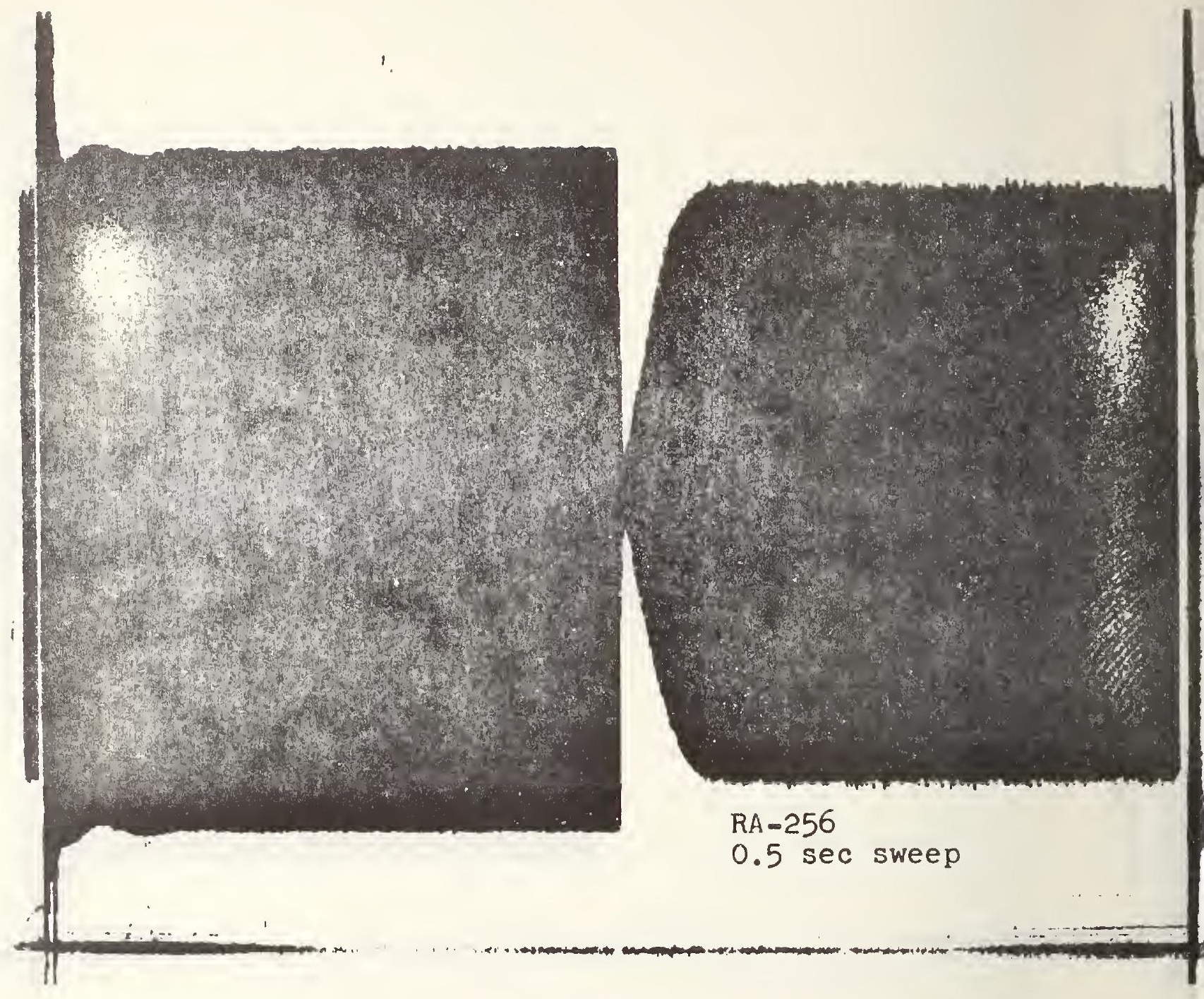




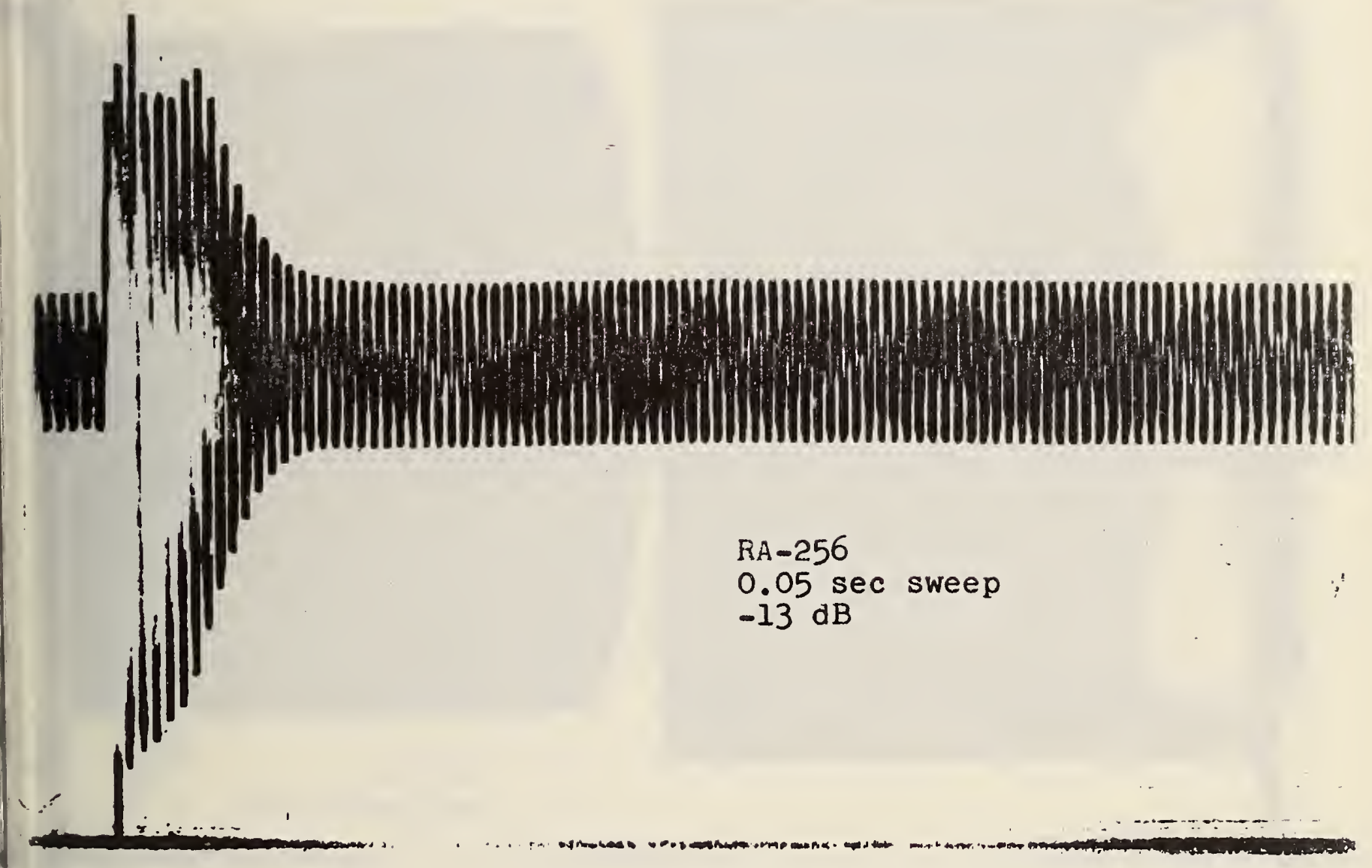




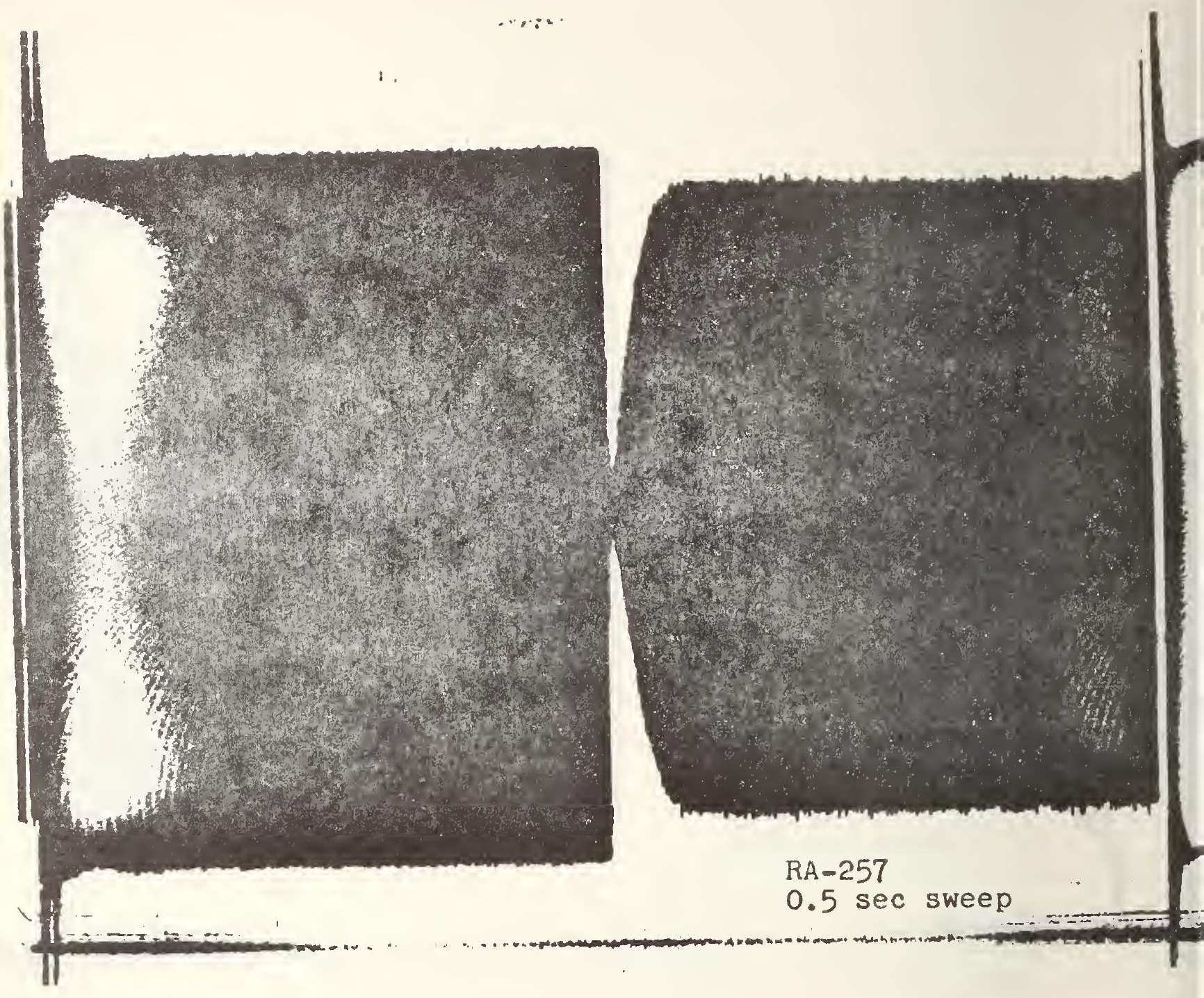




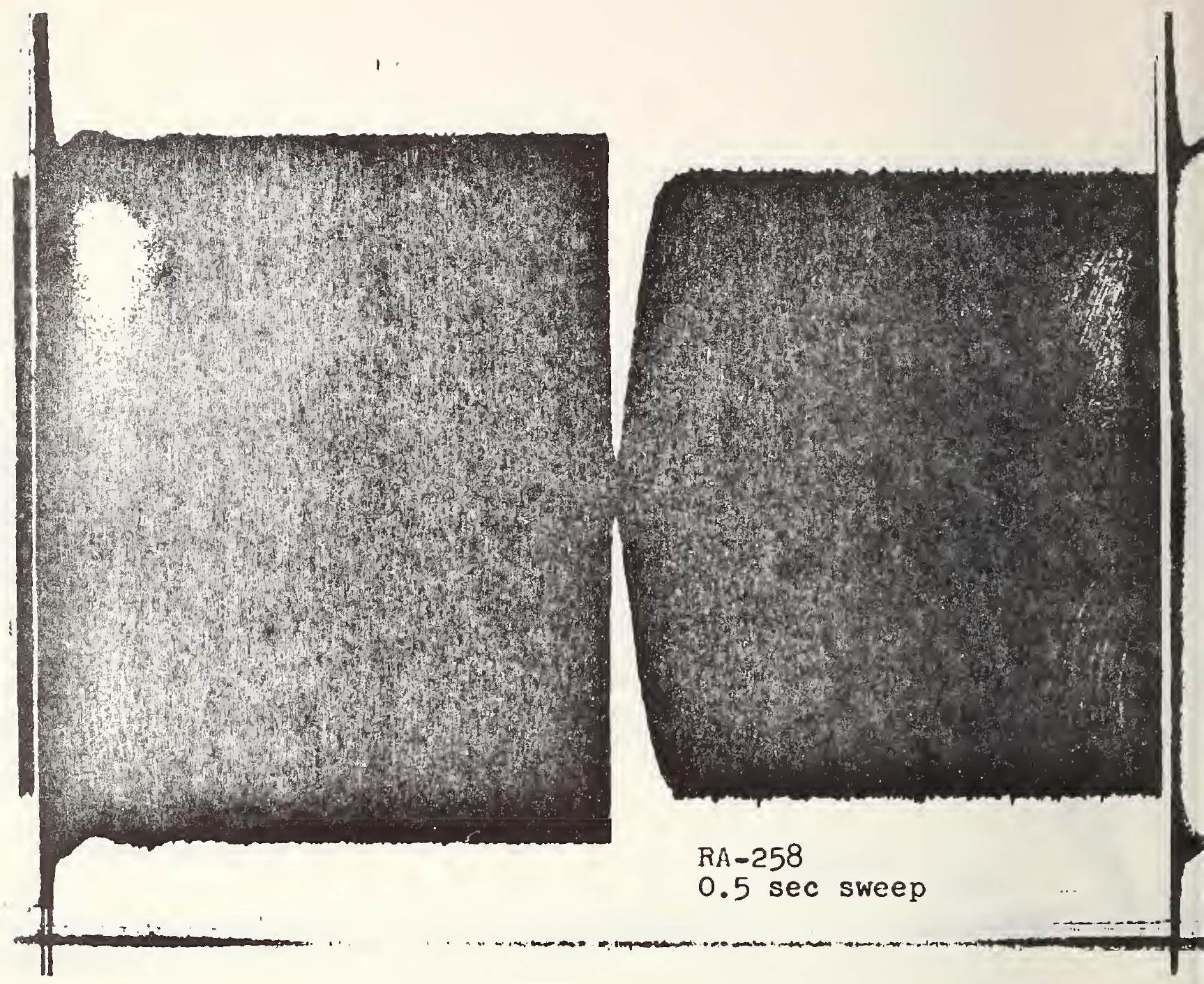




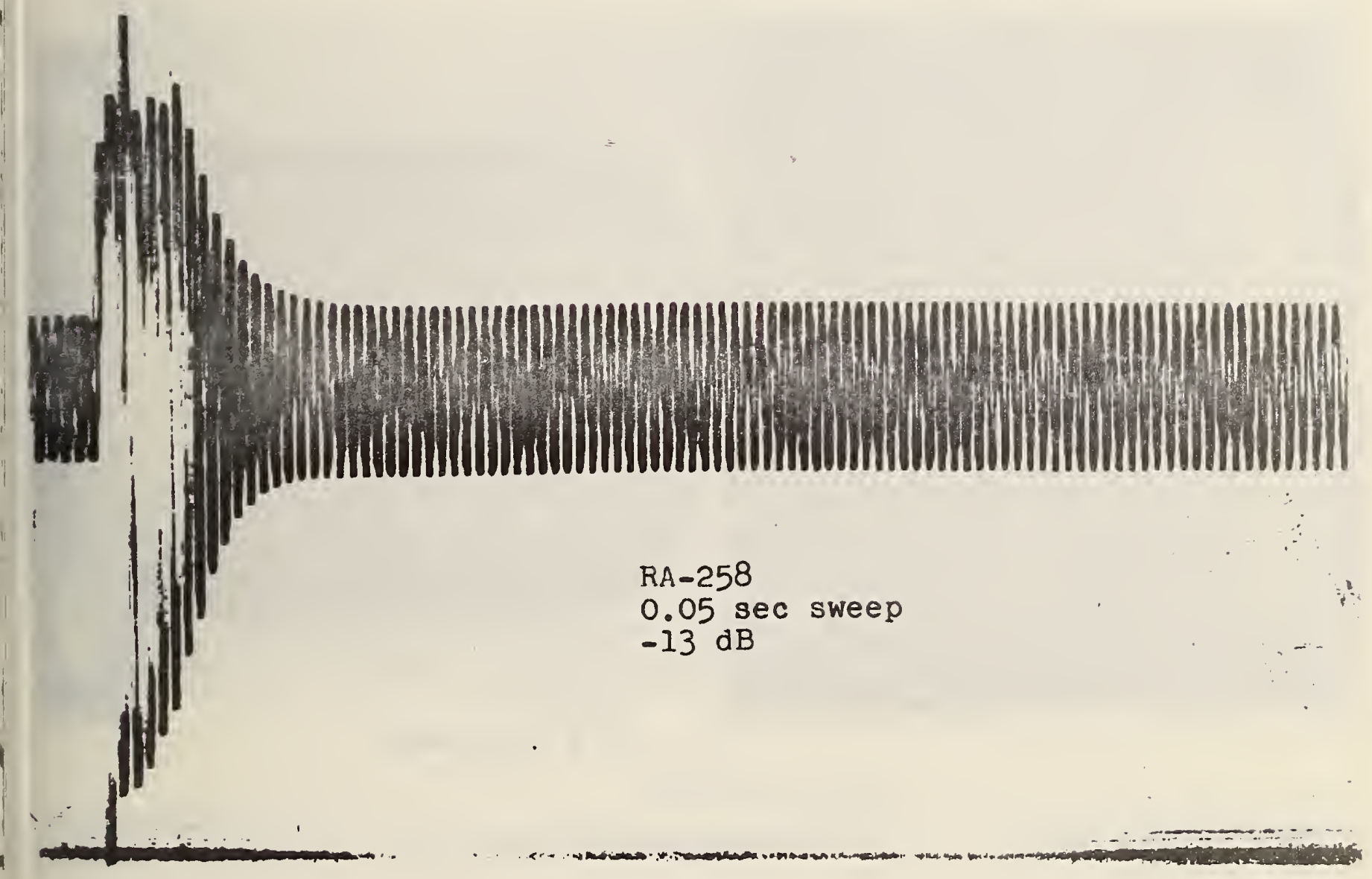



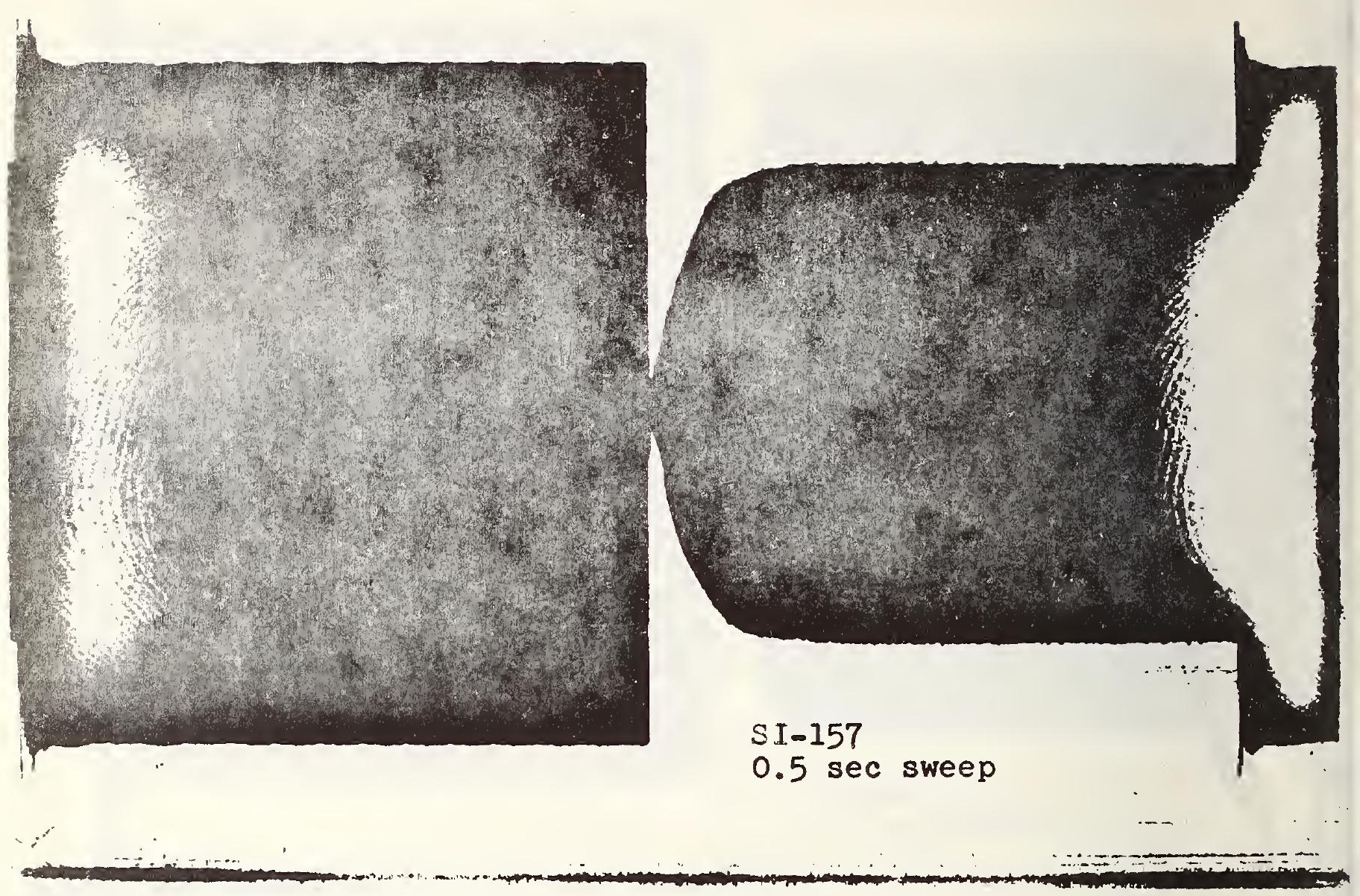

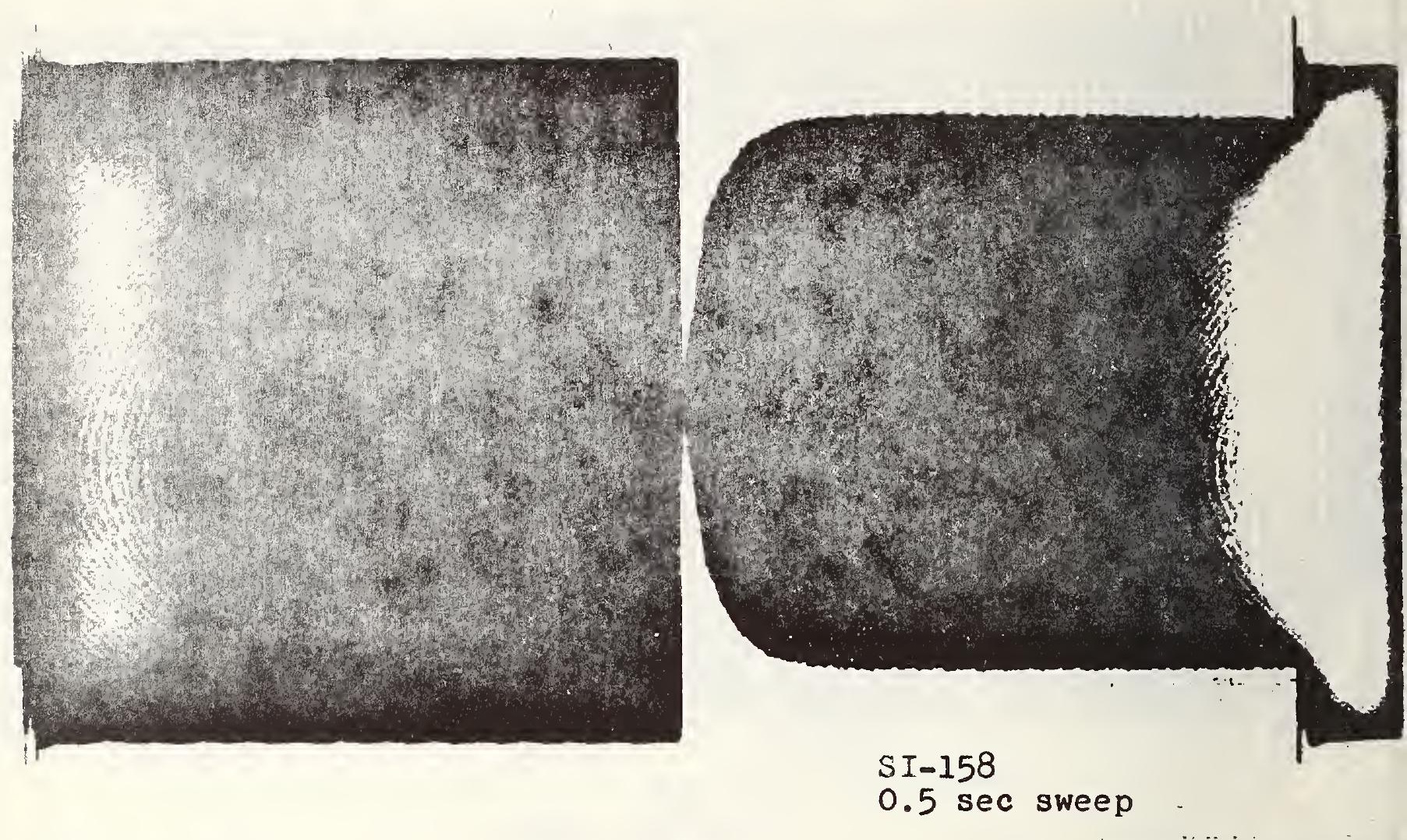

\section{$S I-158$ \\ 0.5 sec sweep}



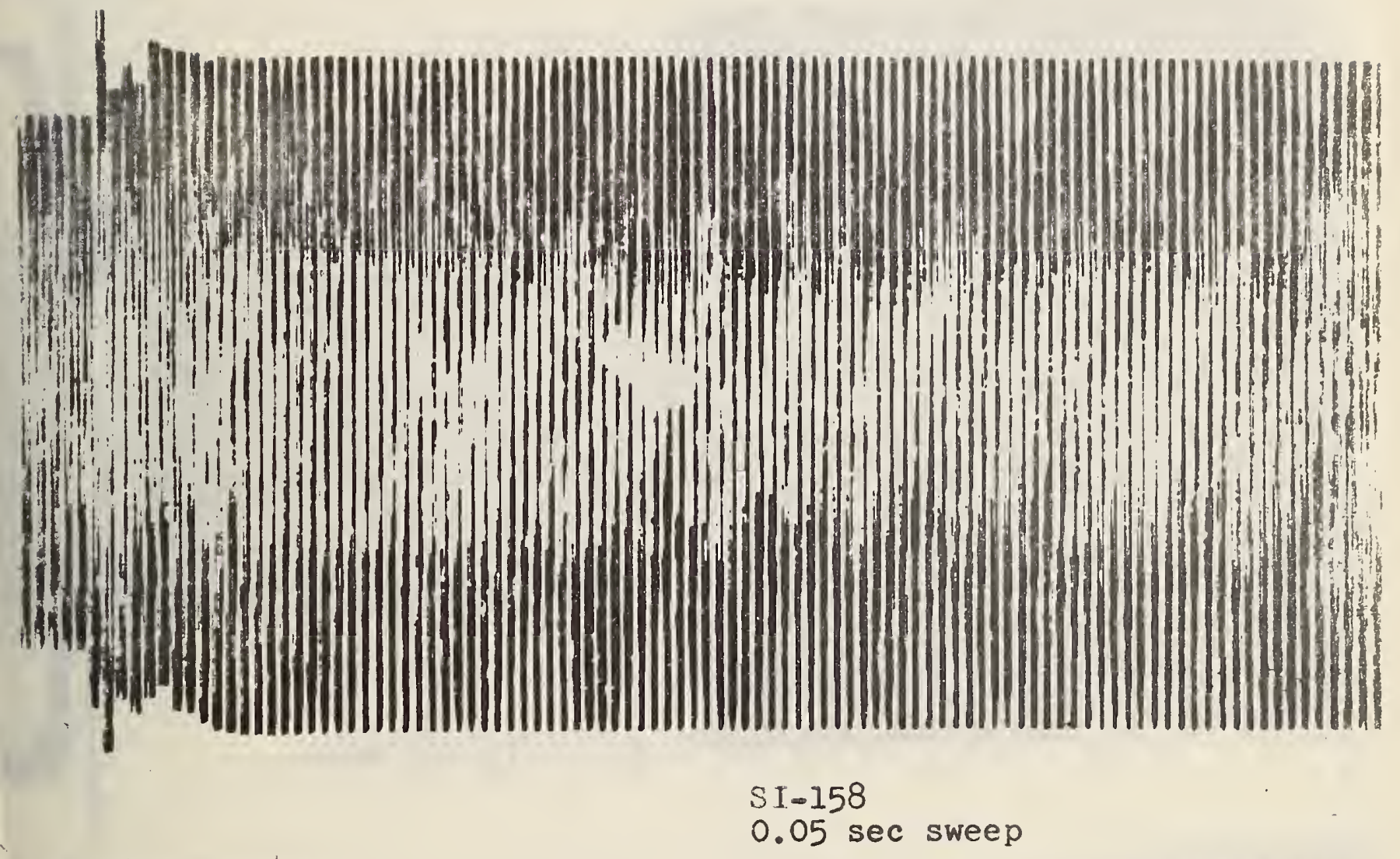


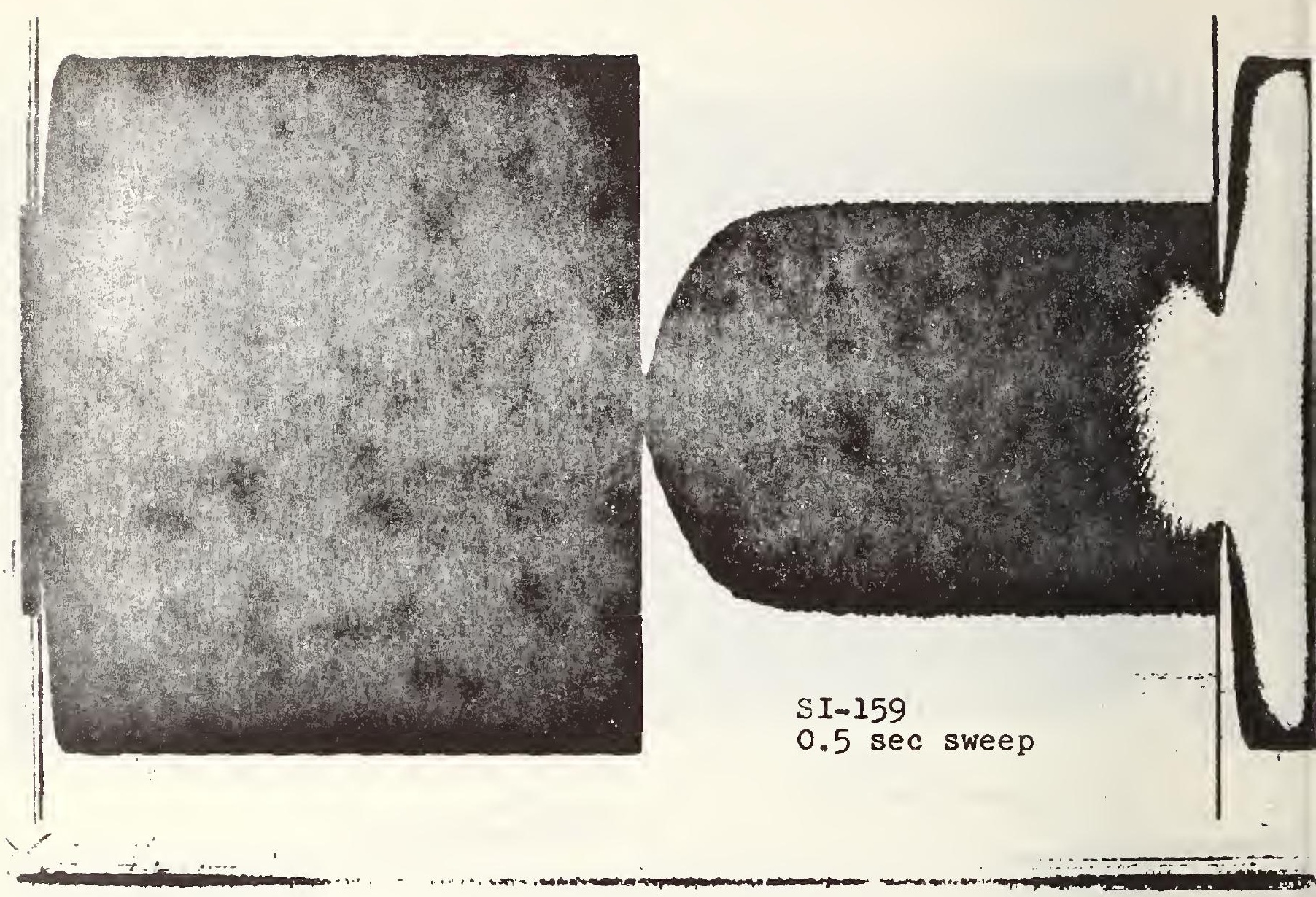




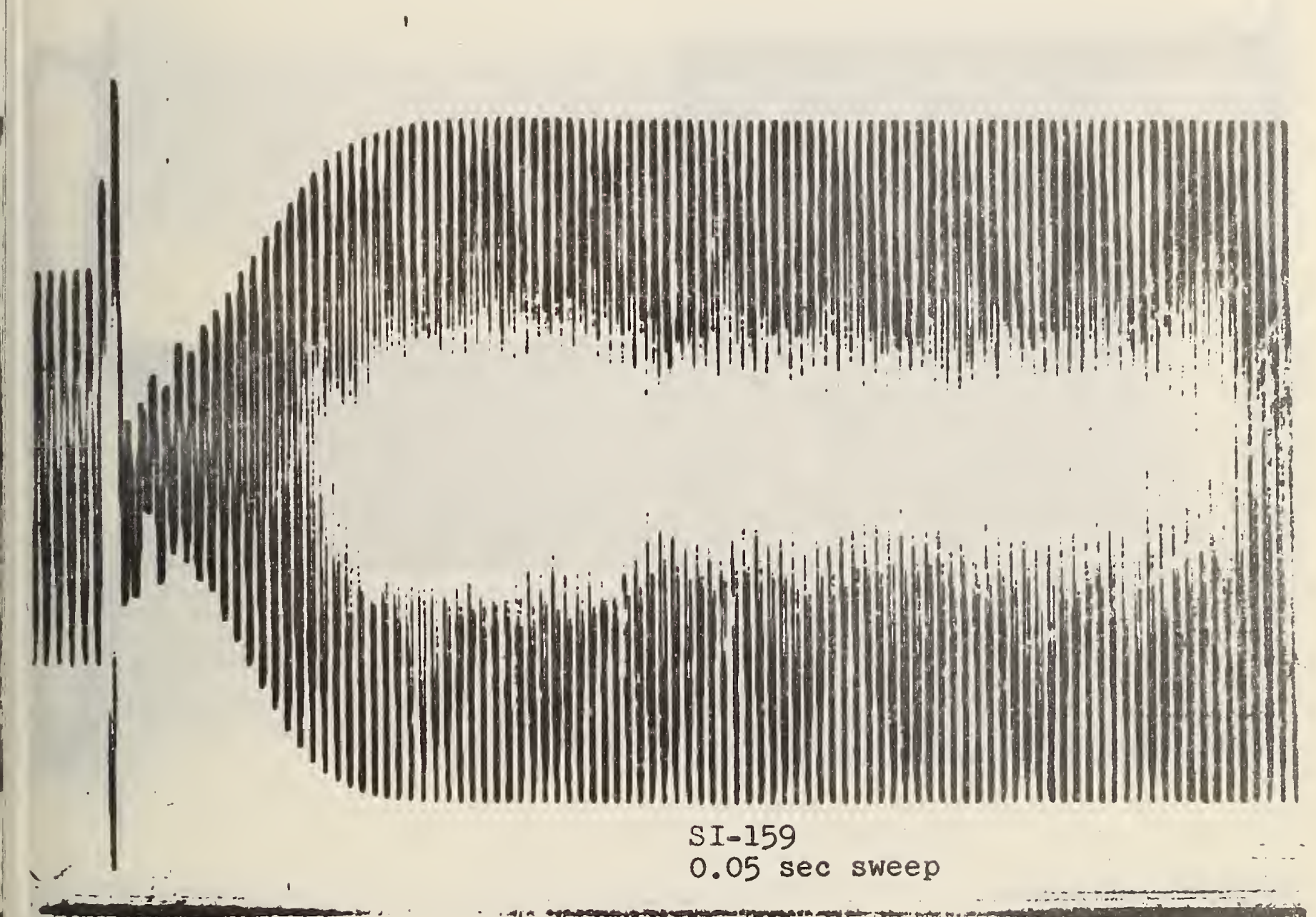




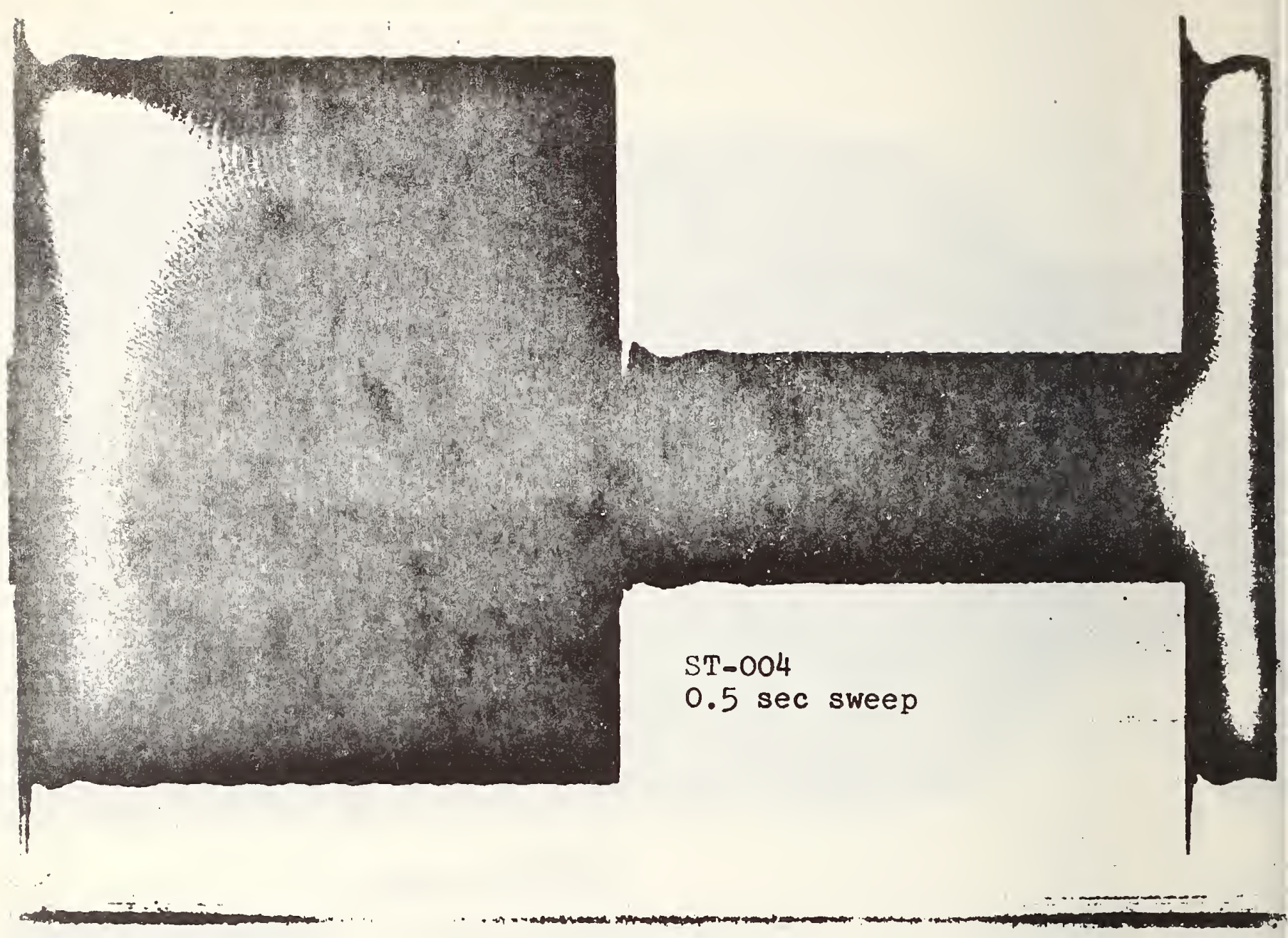



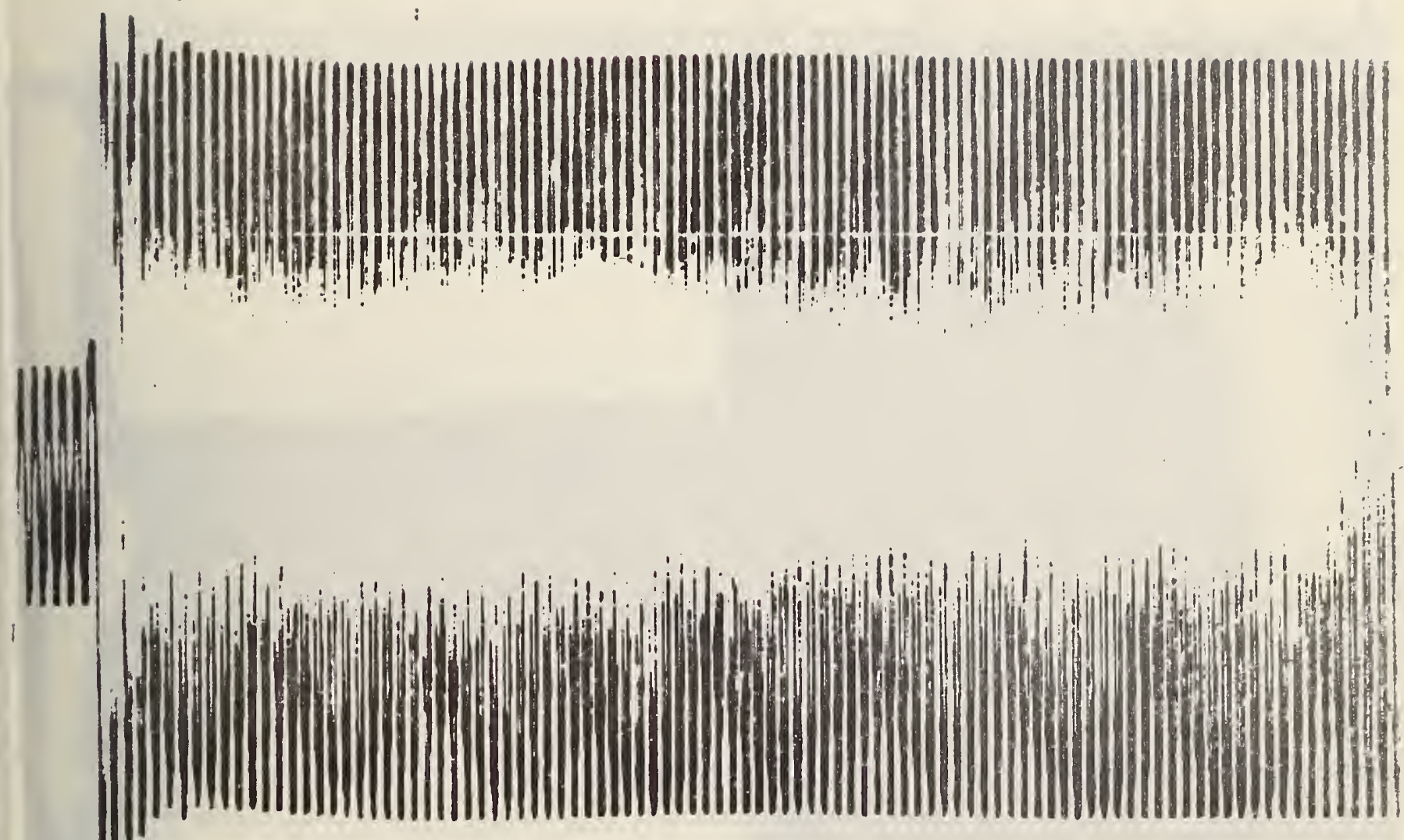

$$
\begin{aligned}
& \text { St }-004 \\
& 0.05 \text { sec sweep }
\end{aligned}
$$




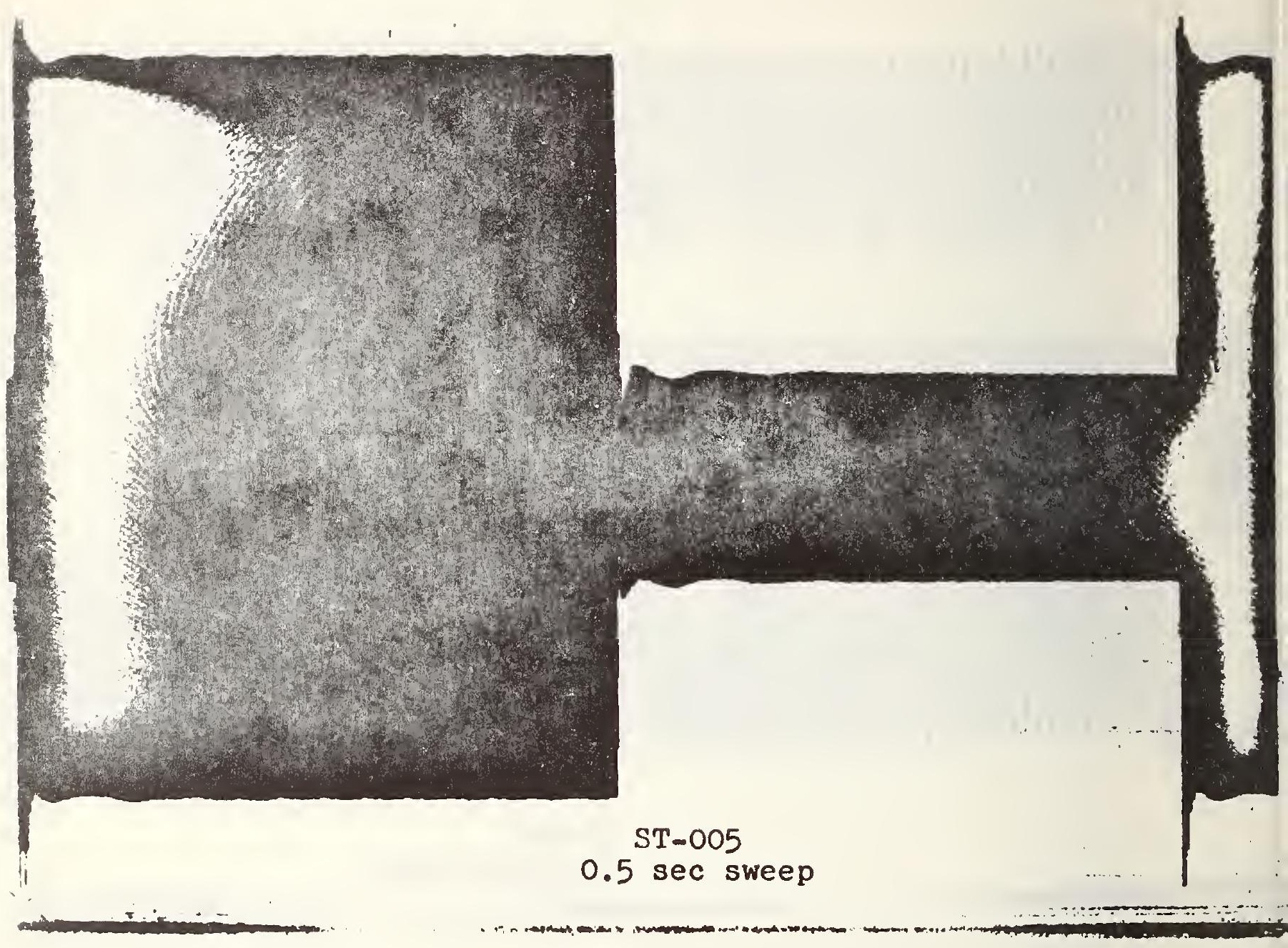



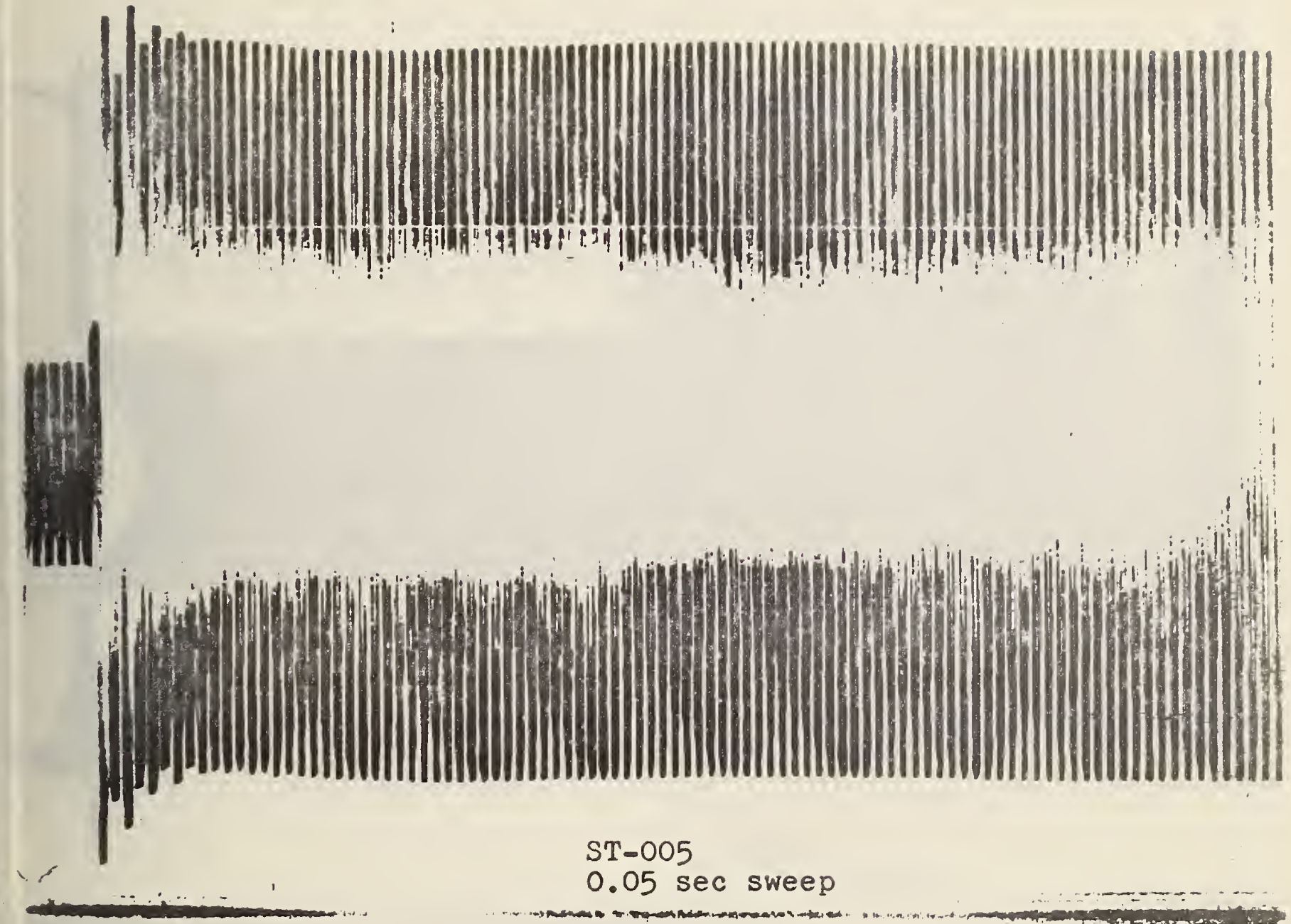


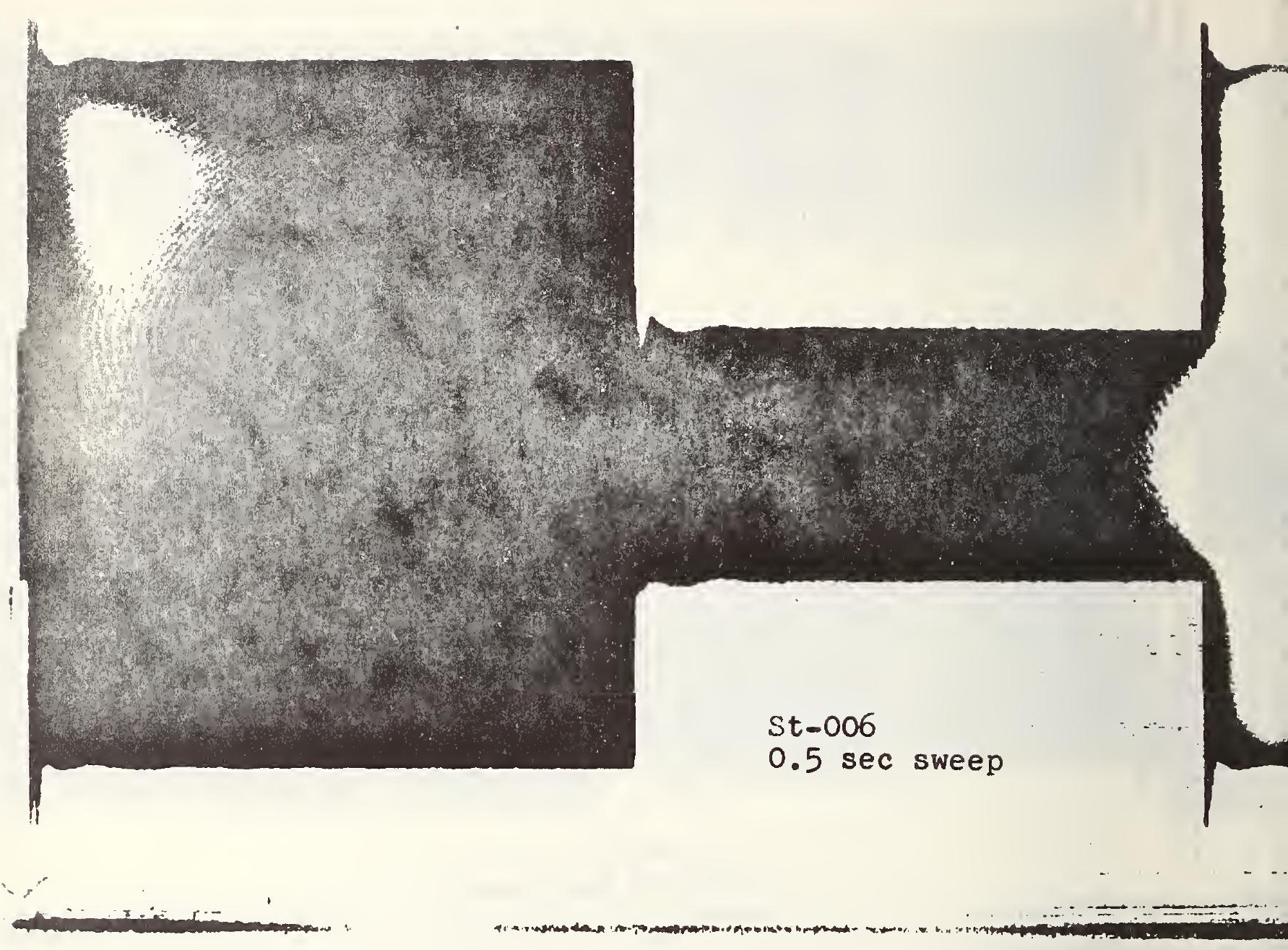




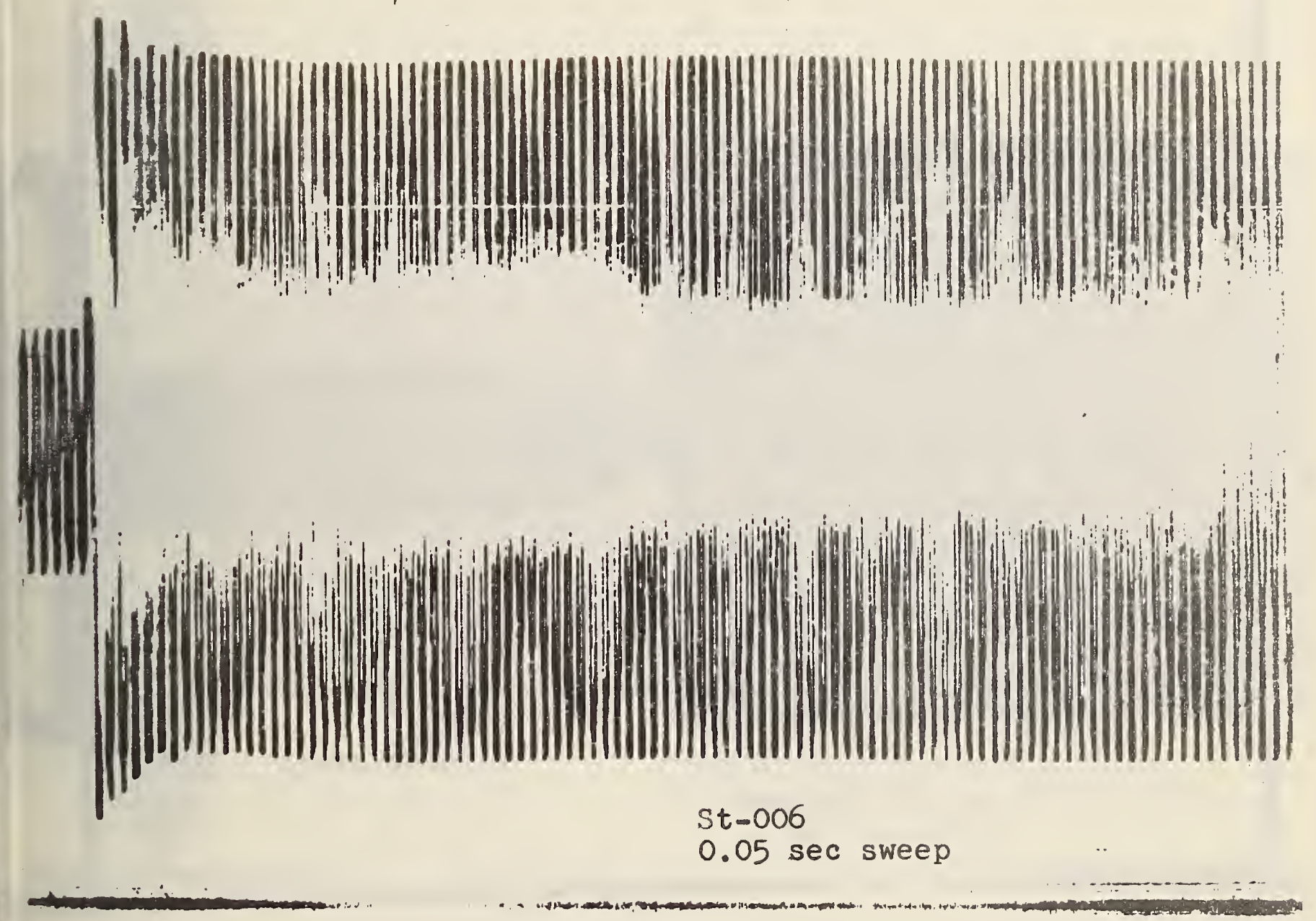




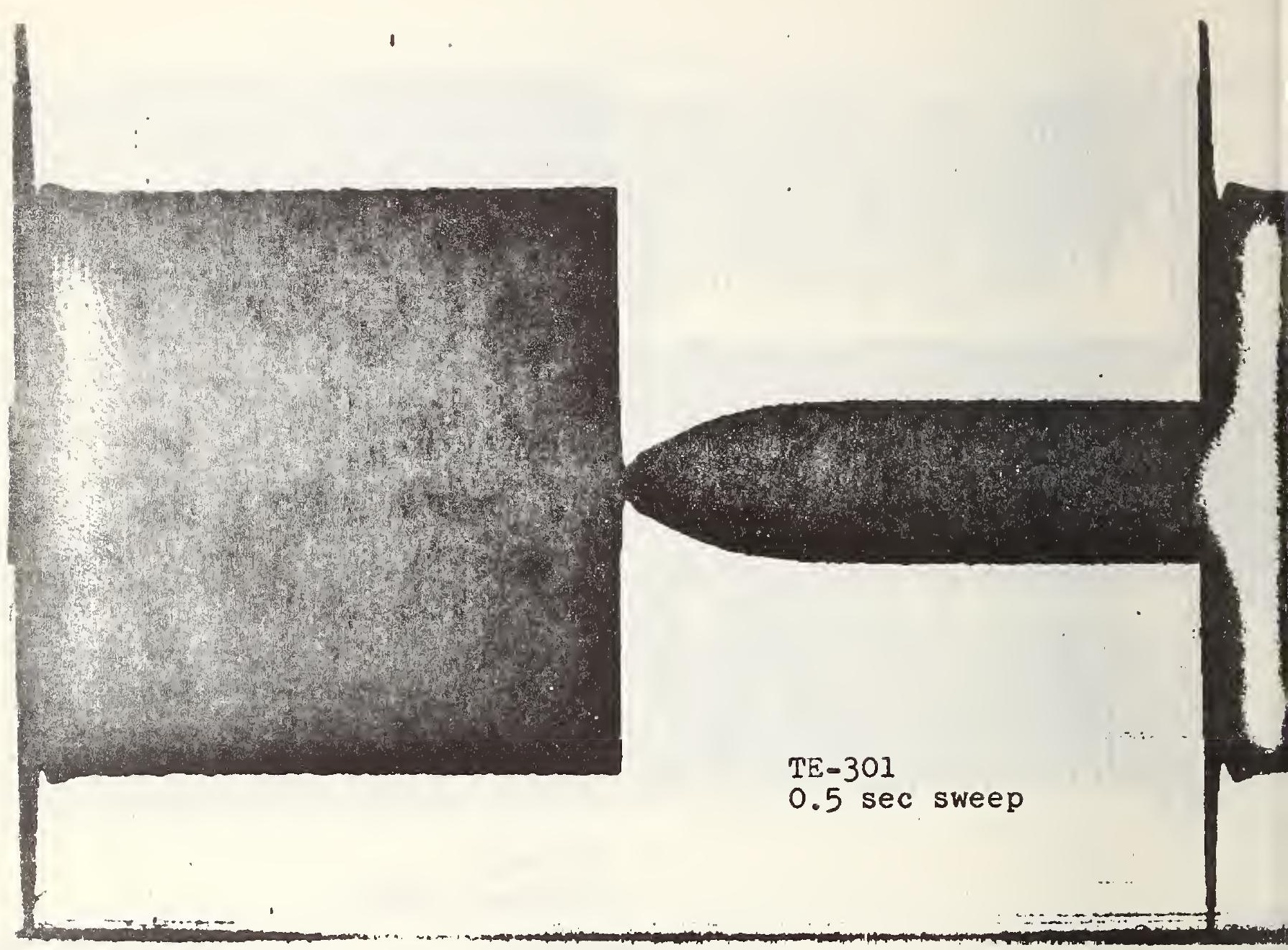




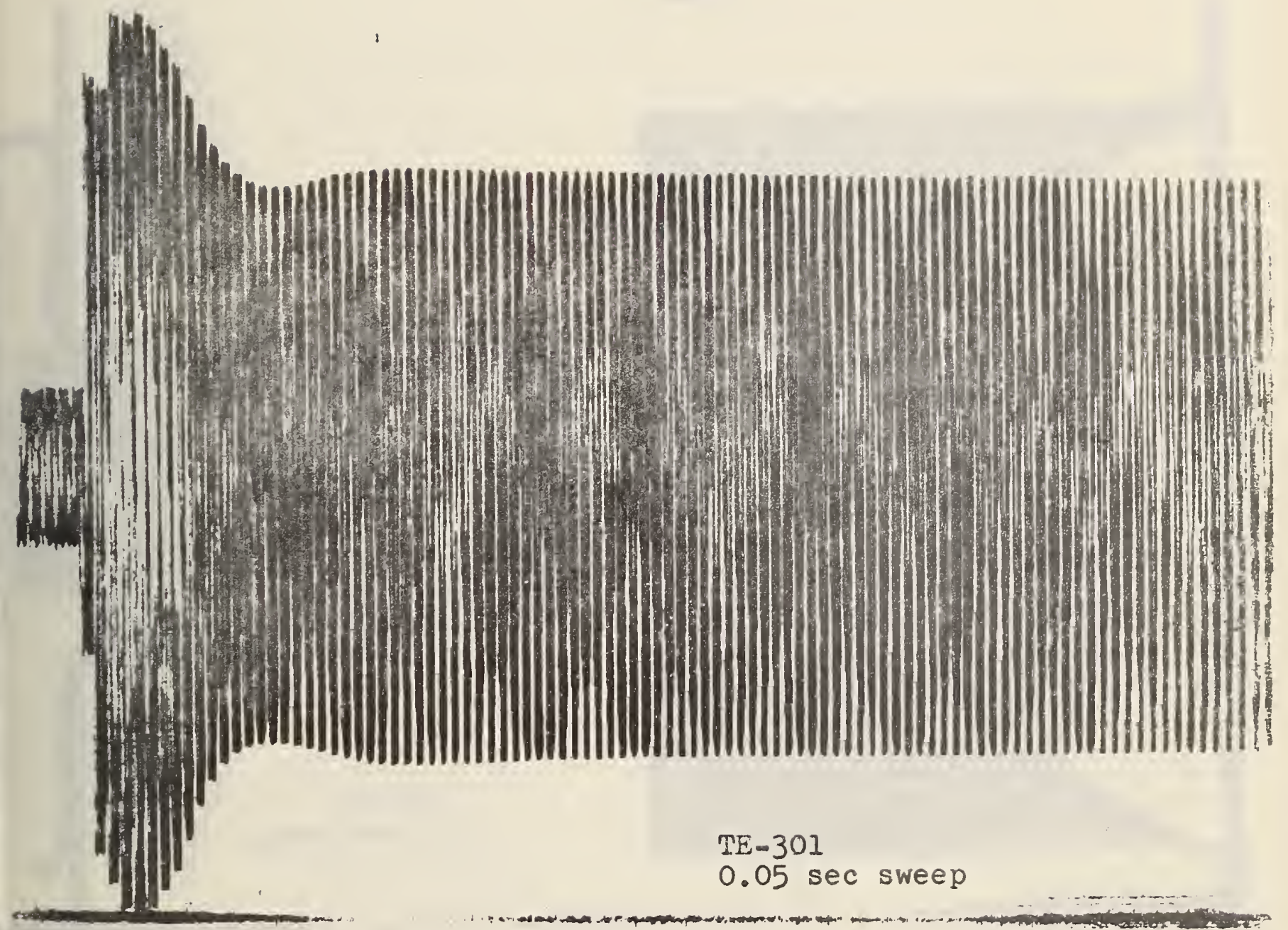




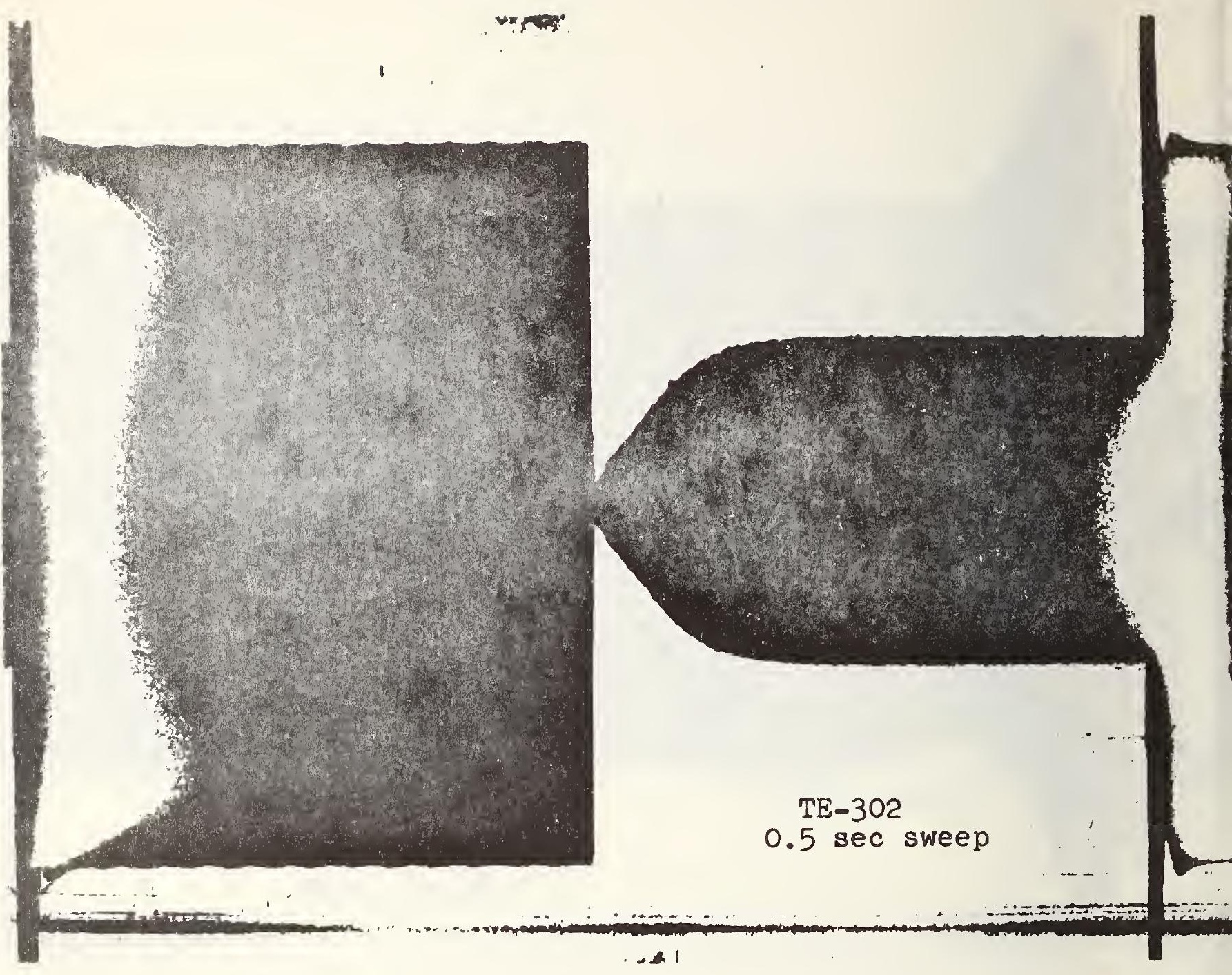




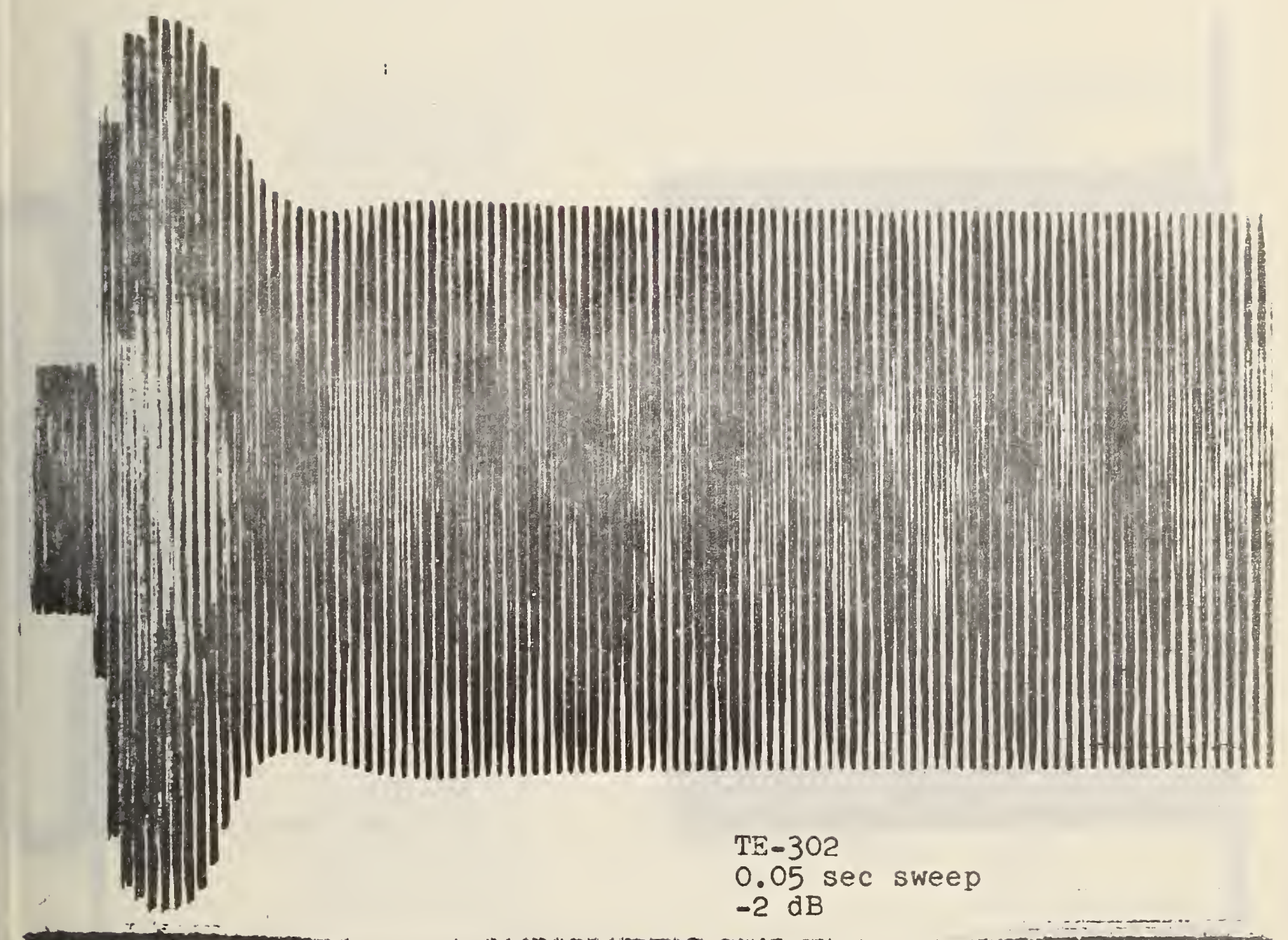




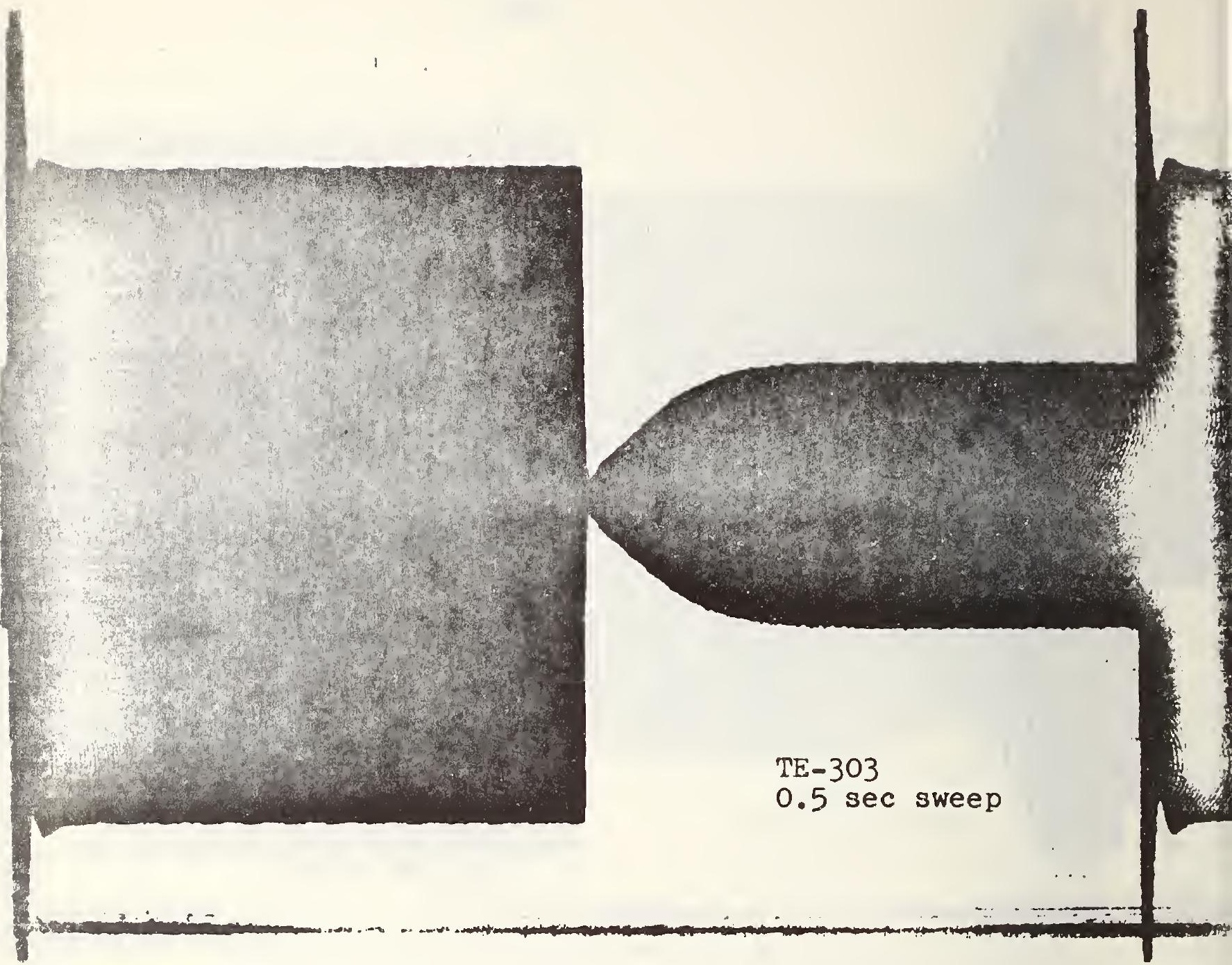




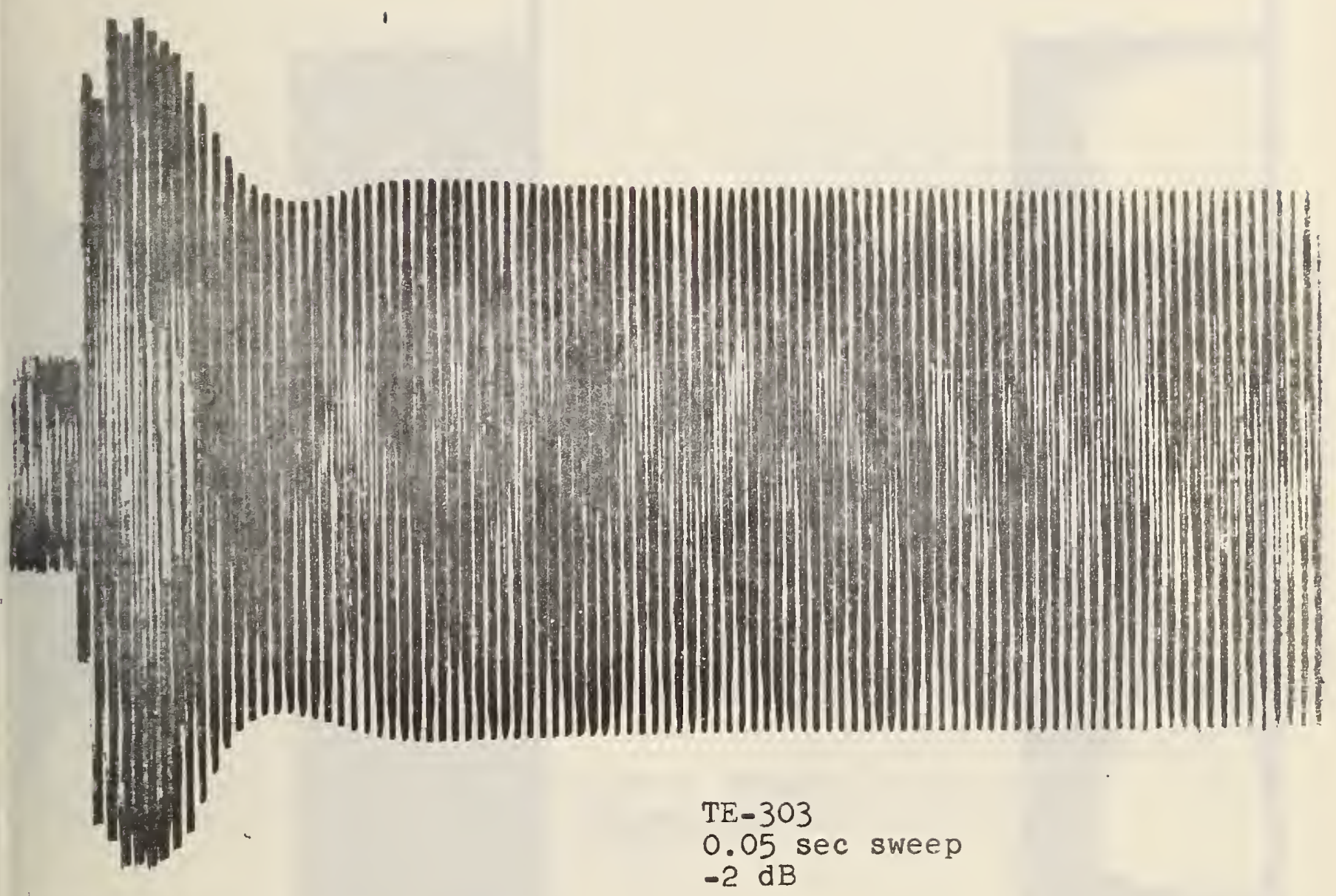

$T E-303$
0.05 sec sweep
$-2 \mathrm{~dB}$ 


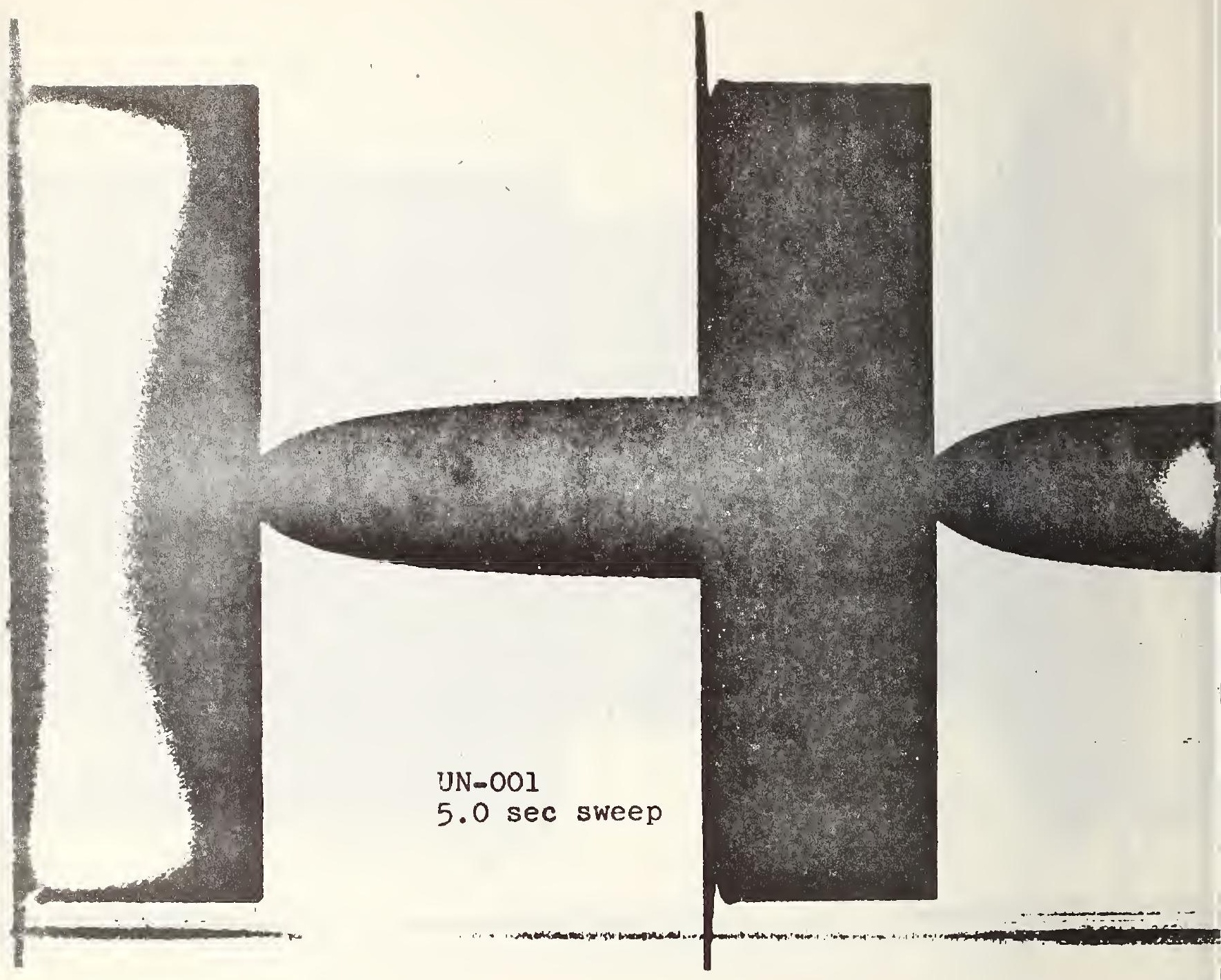




$$
\begin{aligned}
& \text { UN-OOI } \\
& 0.5 \text { sec sweep } \\
& -5 \mathrm{~dB}
\end{aligned}
$$




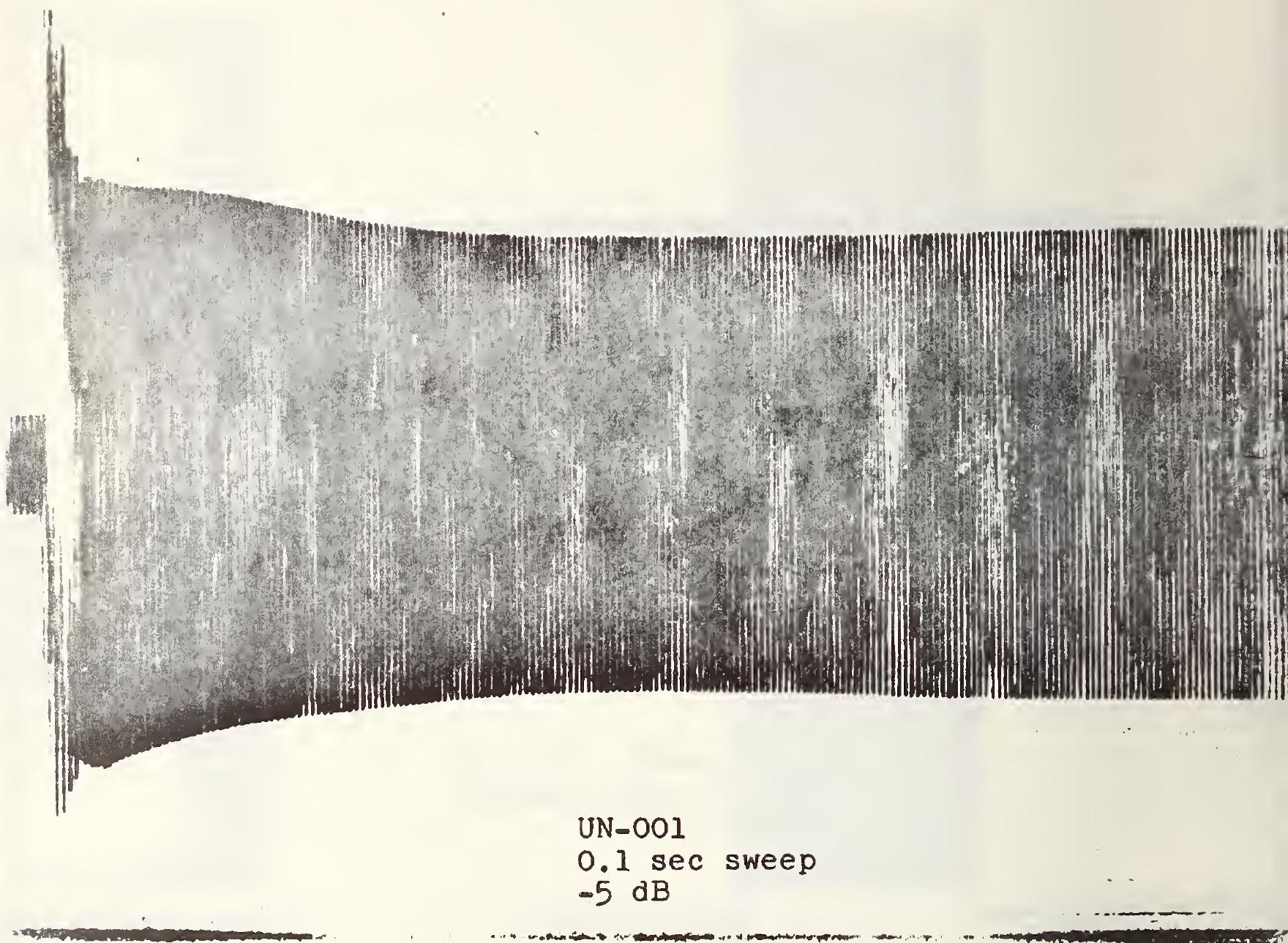




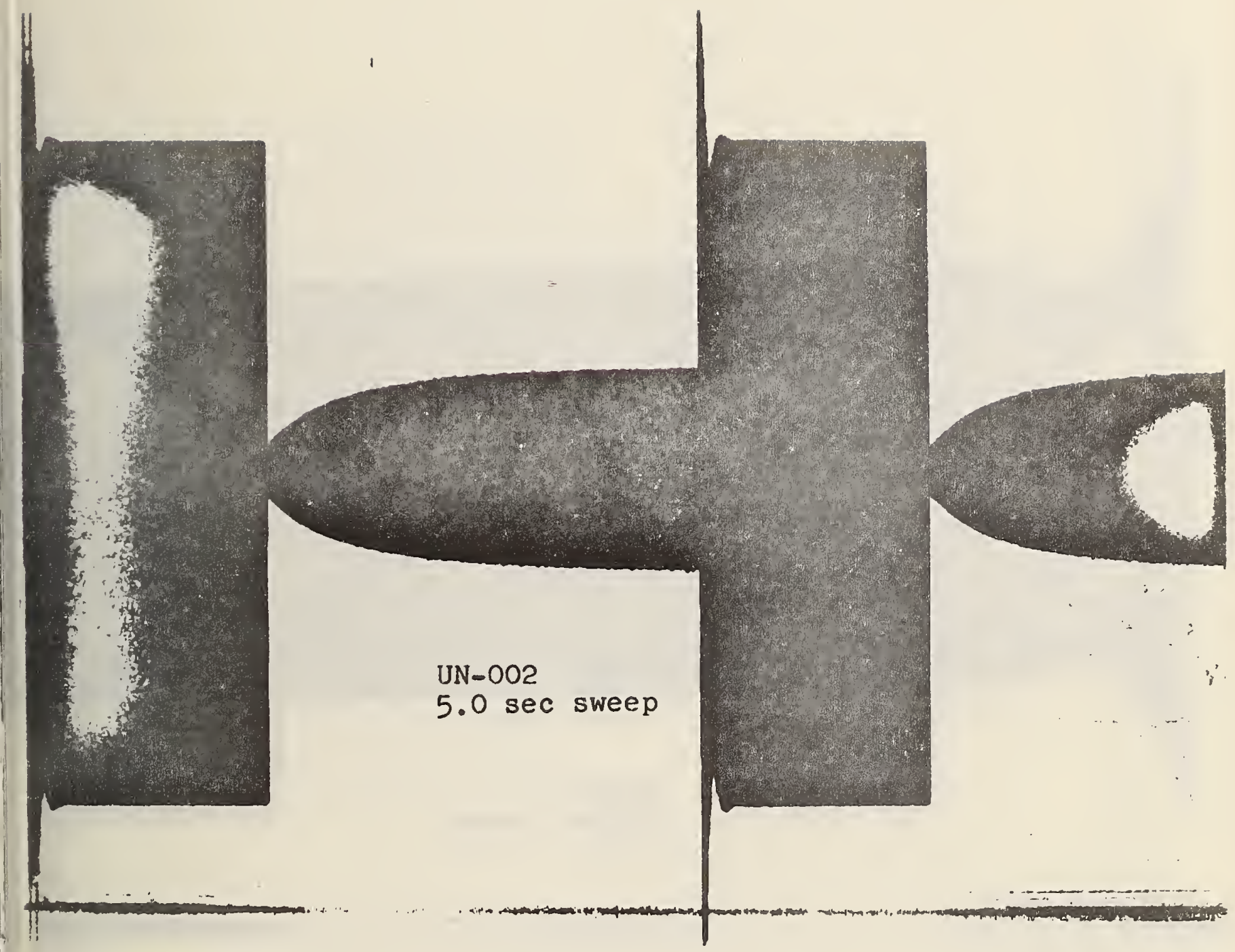



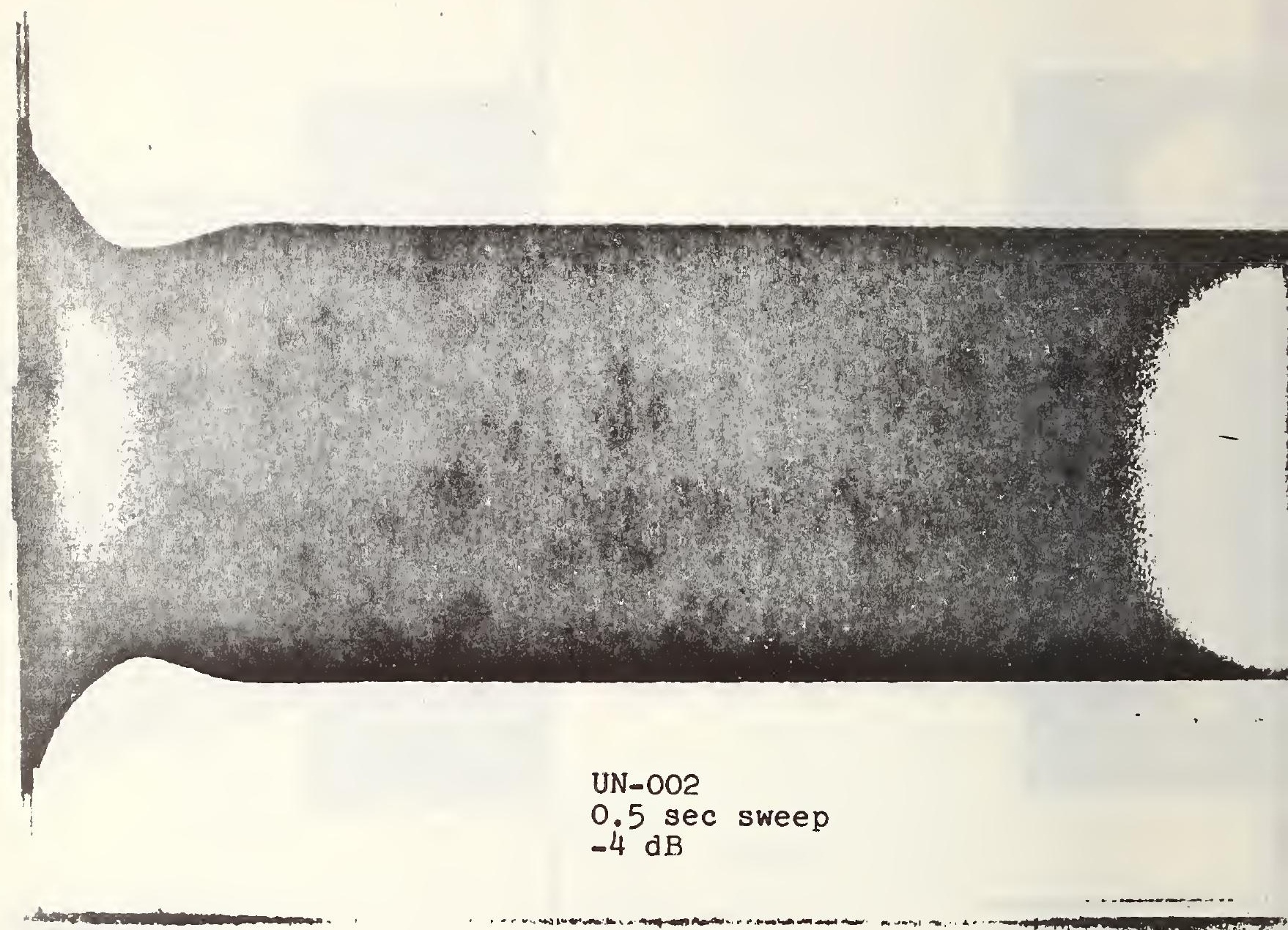


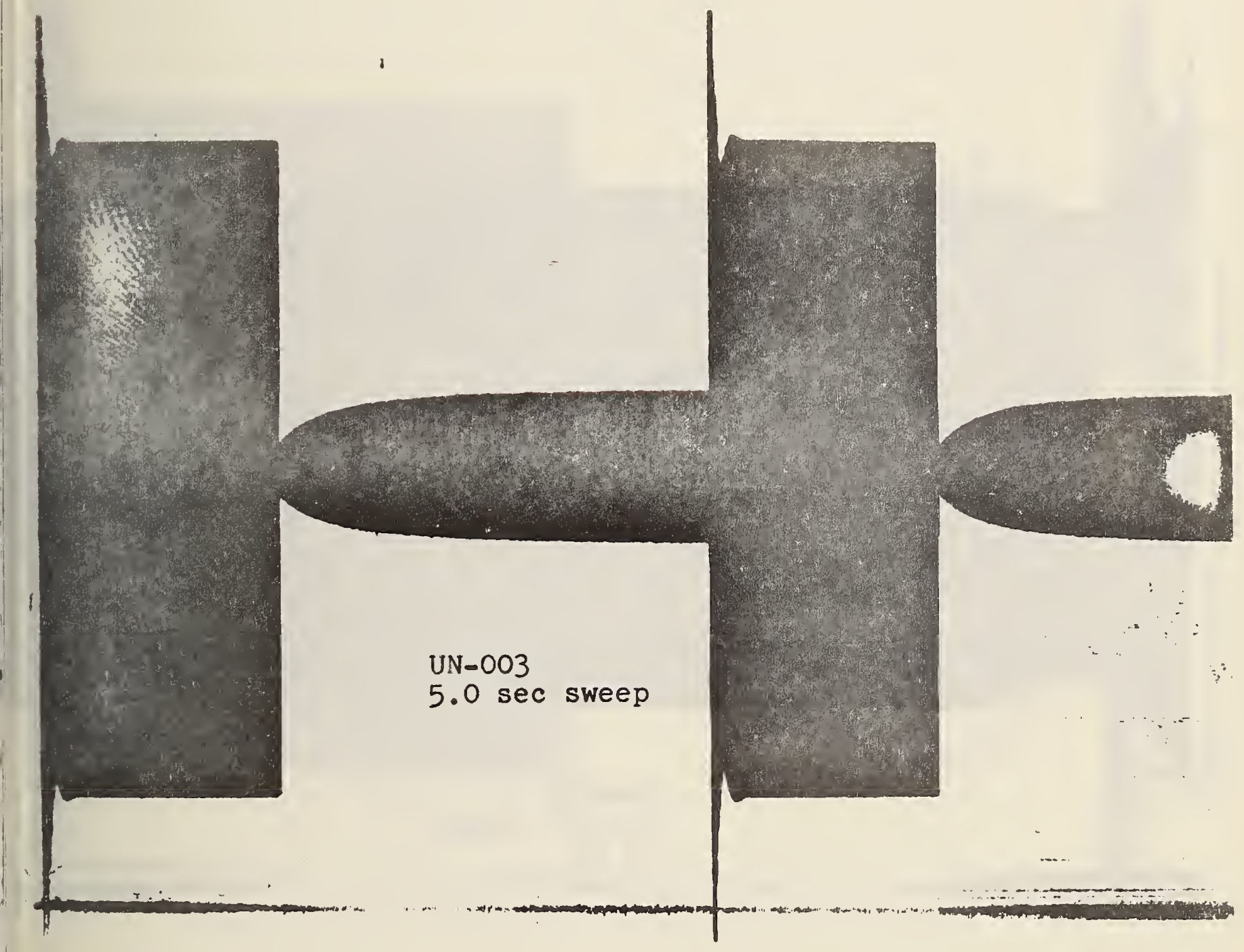



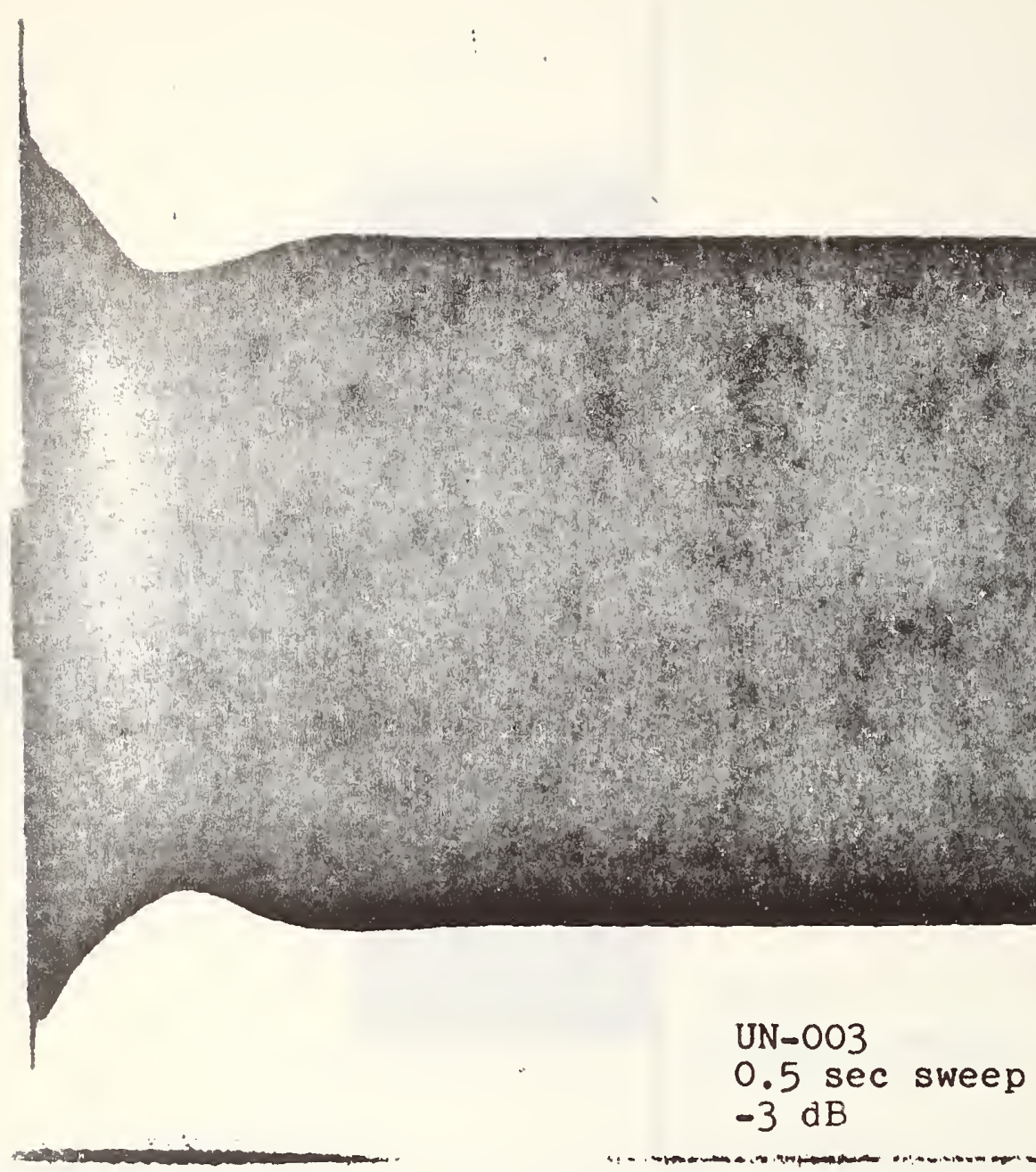


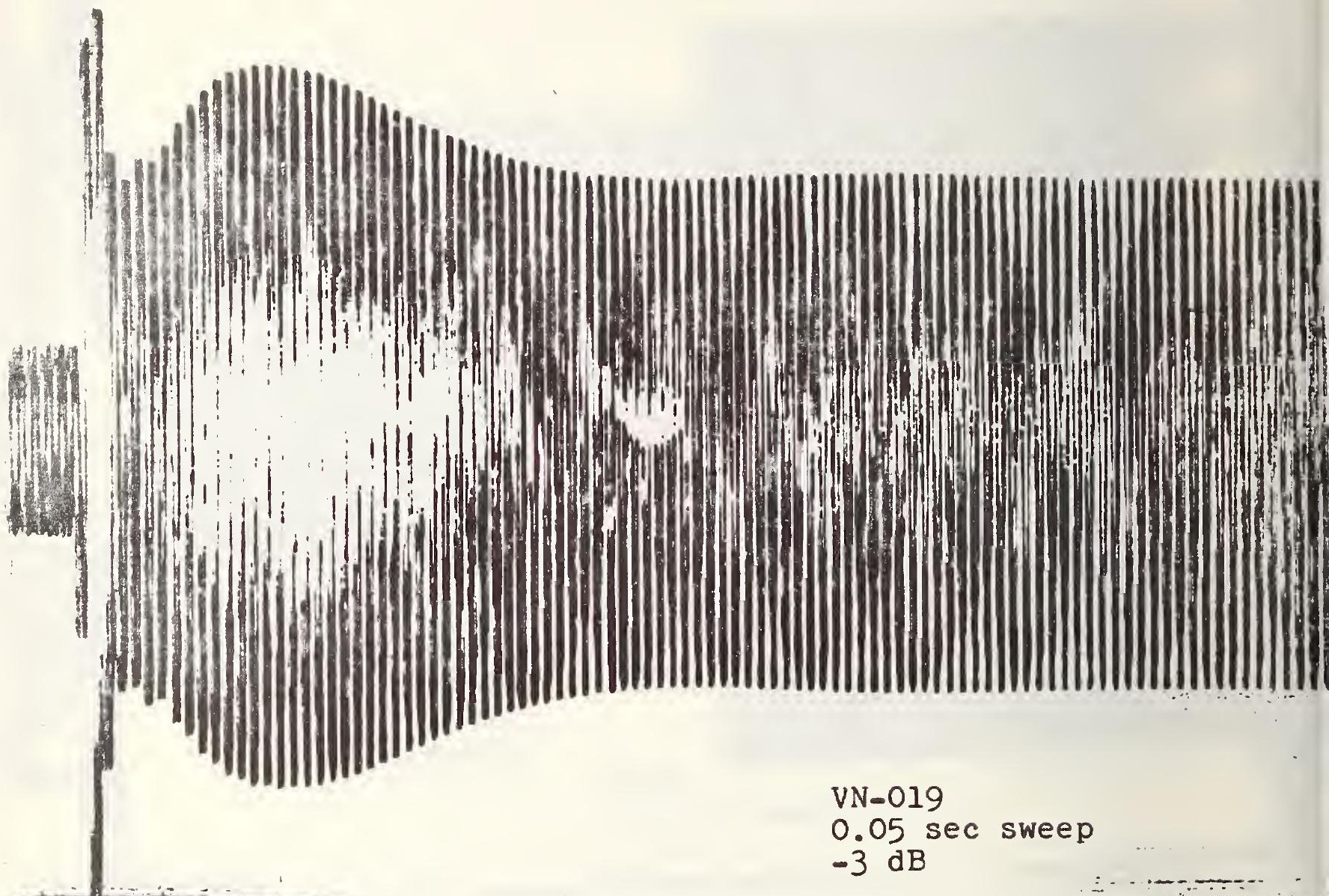




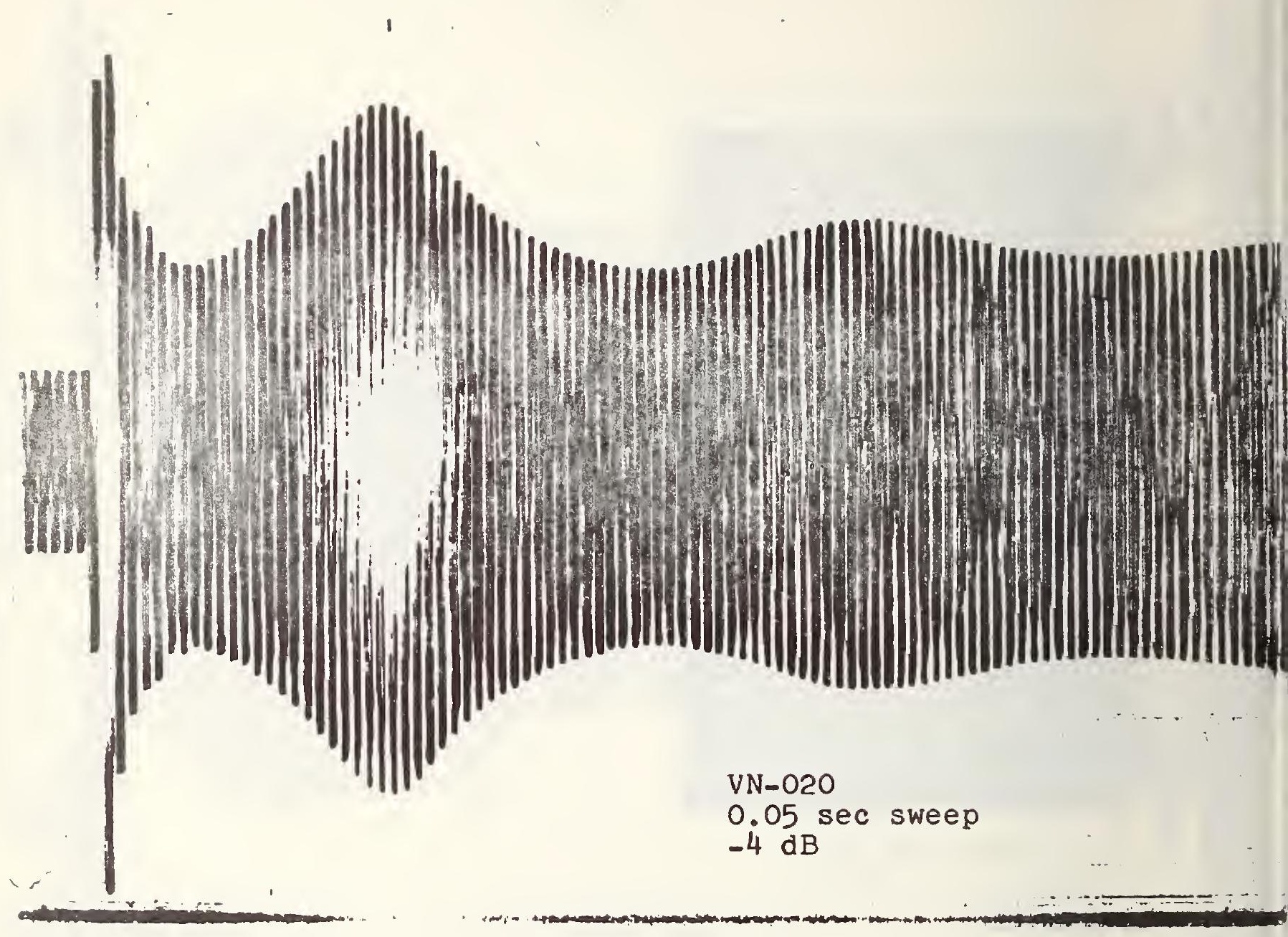




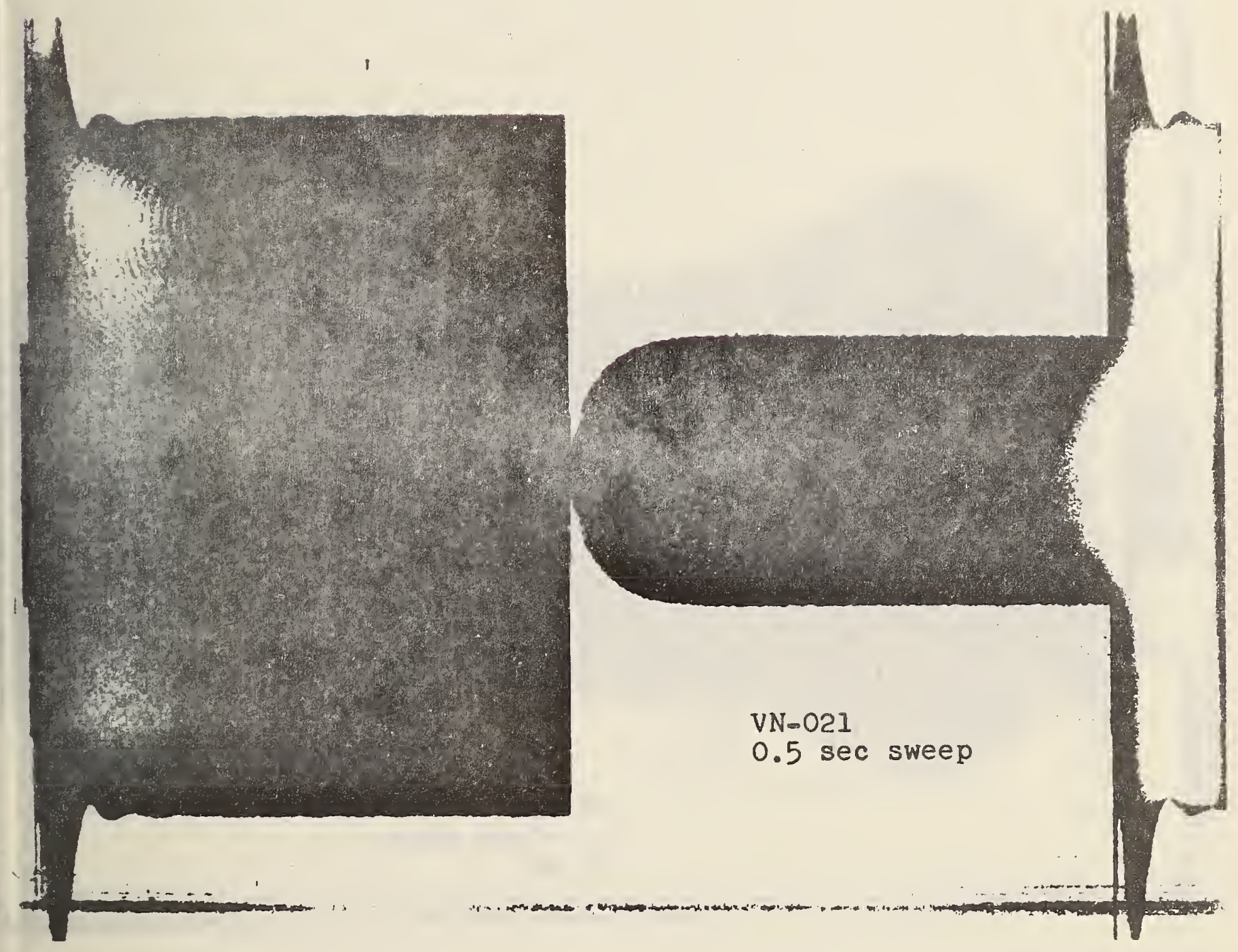




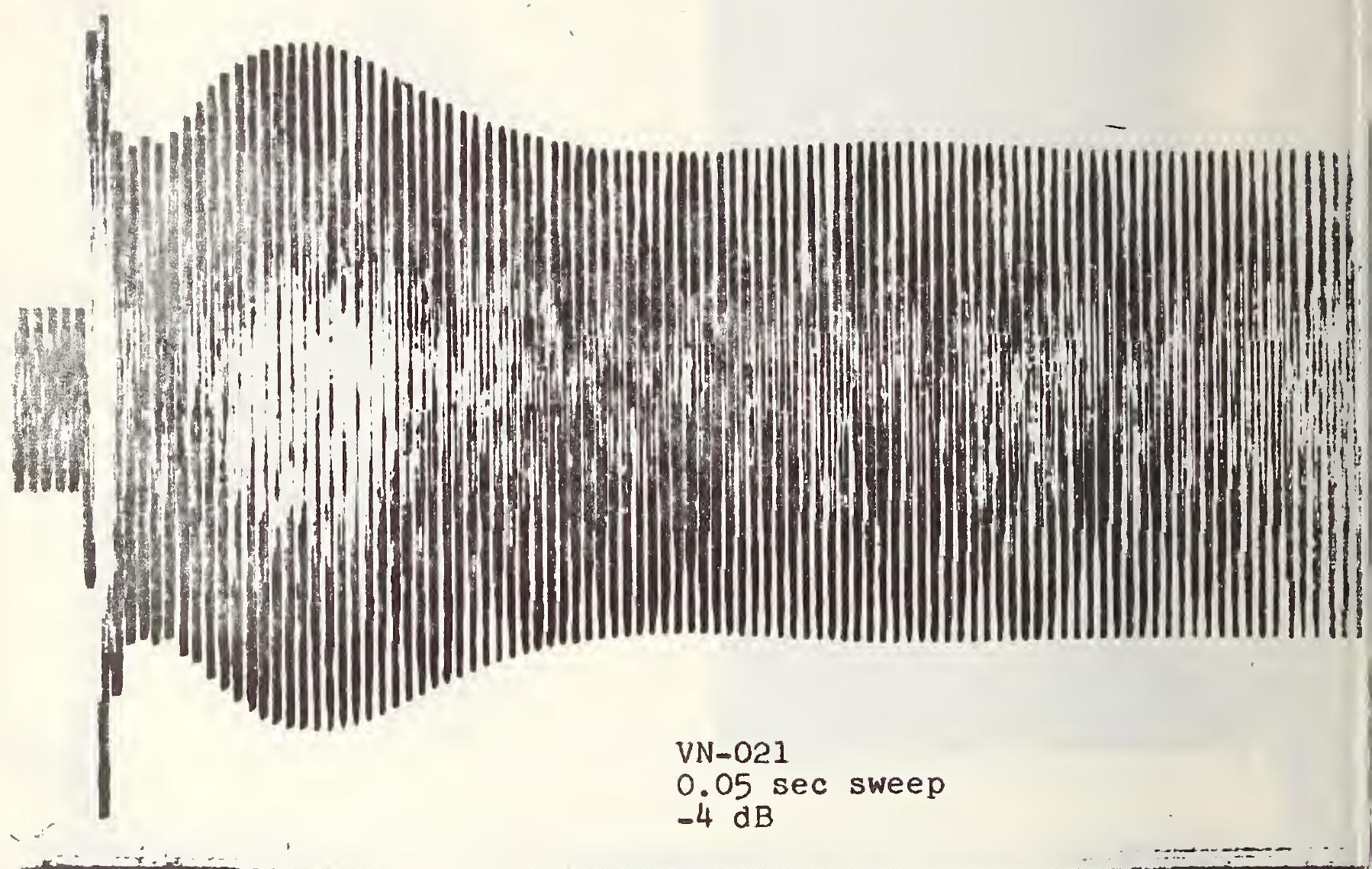




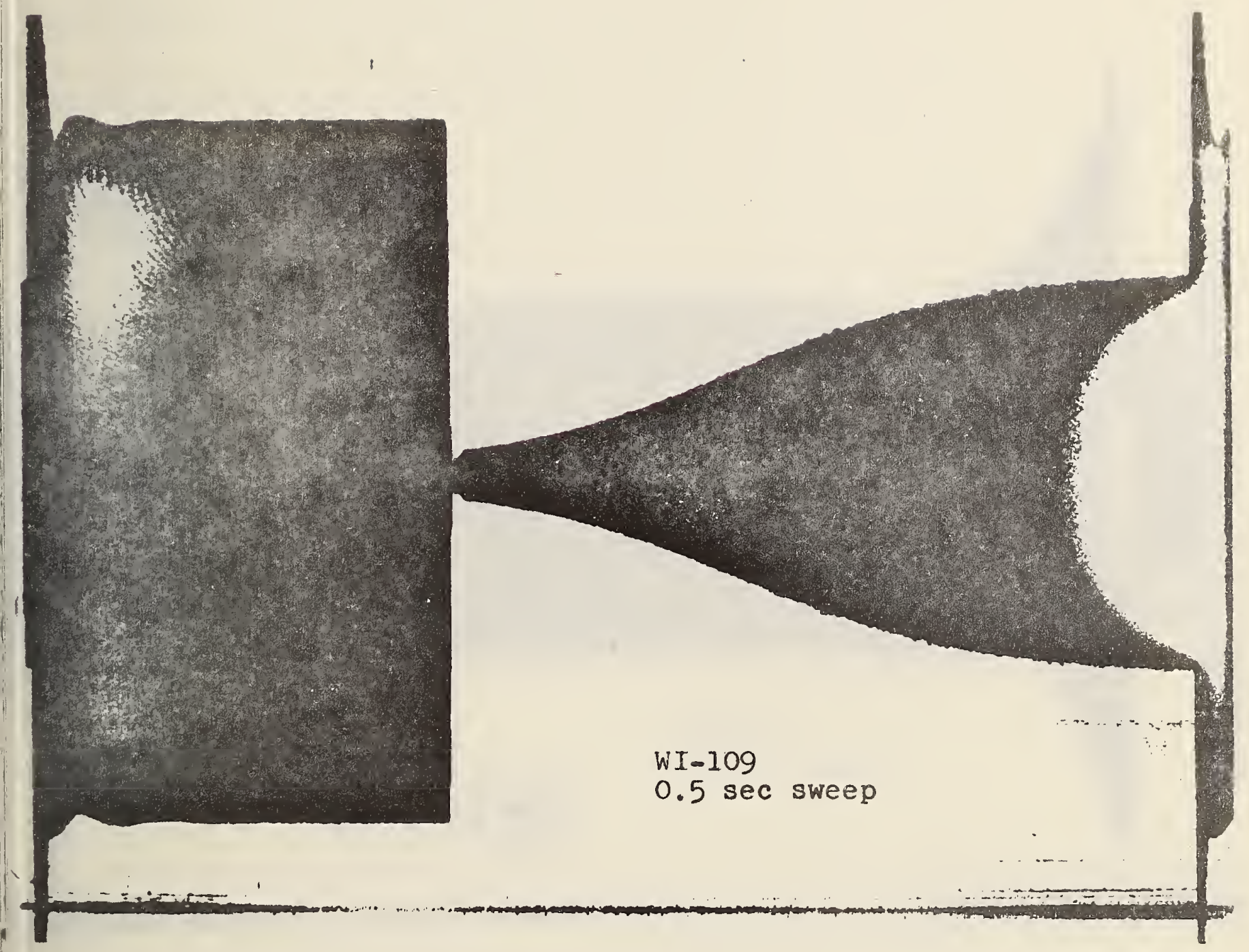




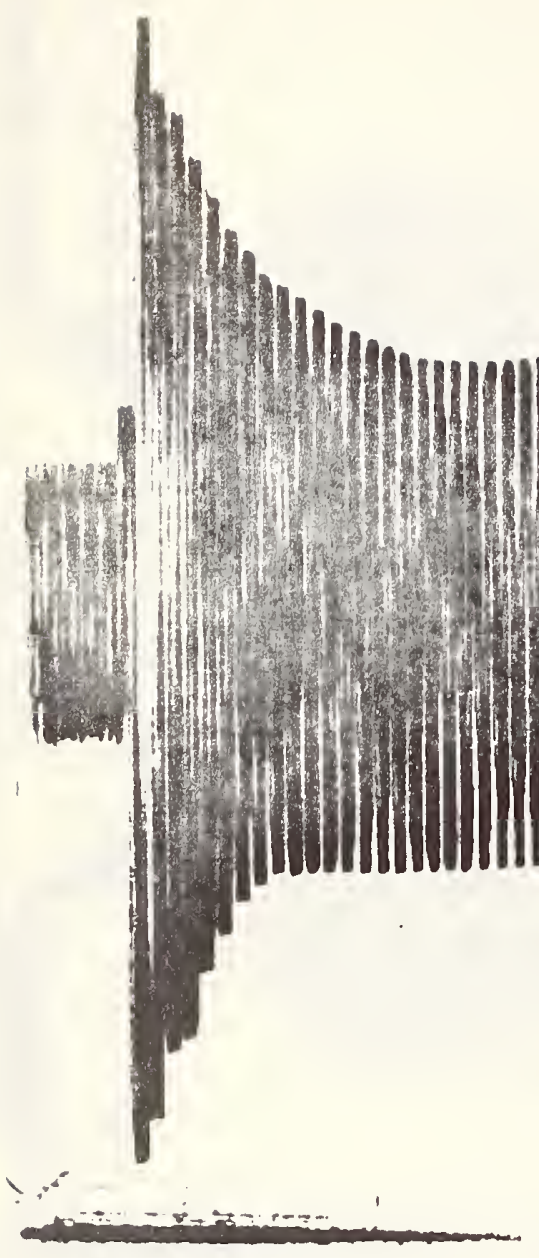

$$
\begin{aligned}
& \text { W } I 09 \\
& 0.05 \text { sec sweep } \\
& -6 \mathrm{~dB}
\end{aligned}
$$




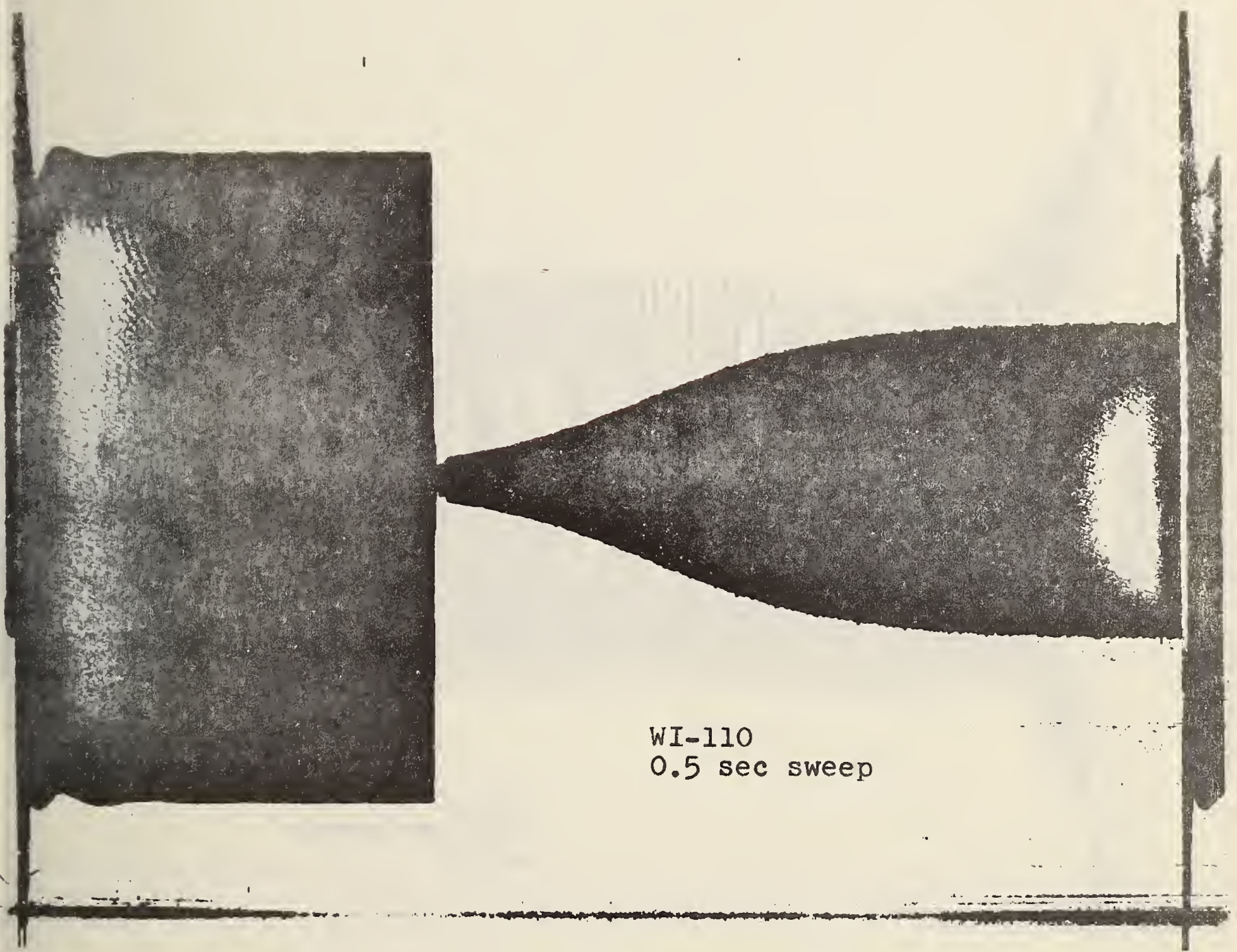




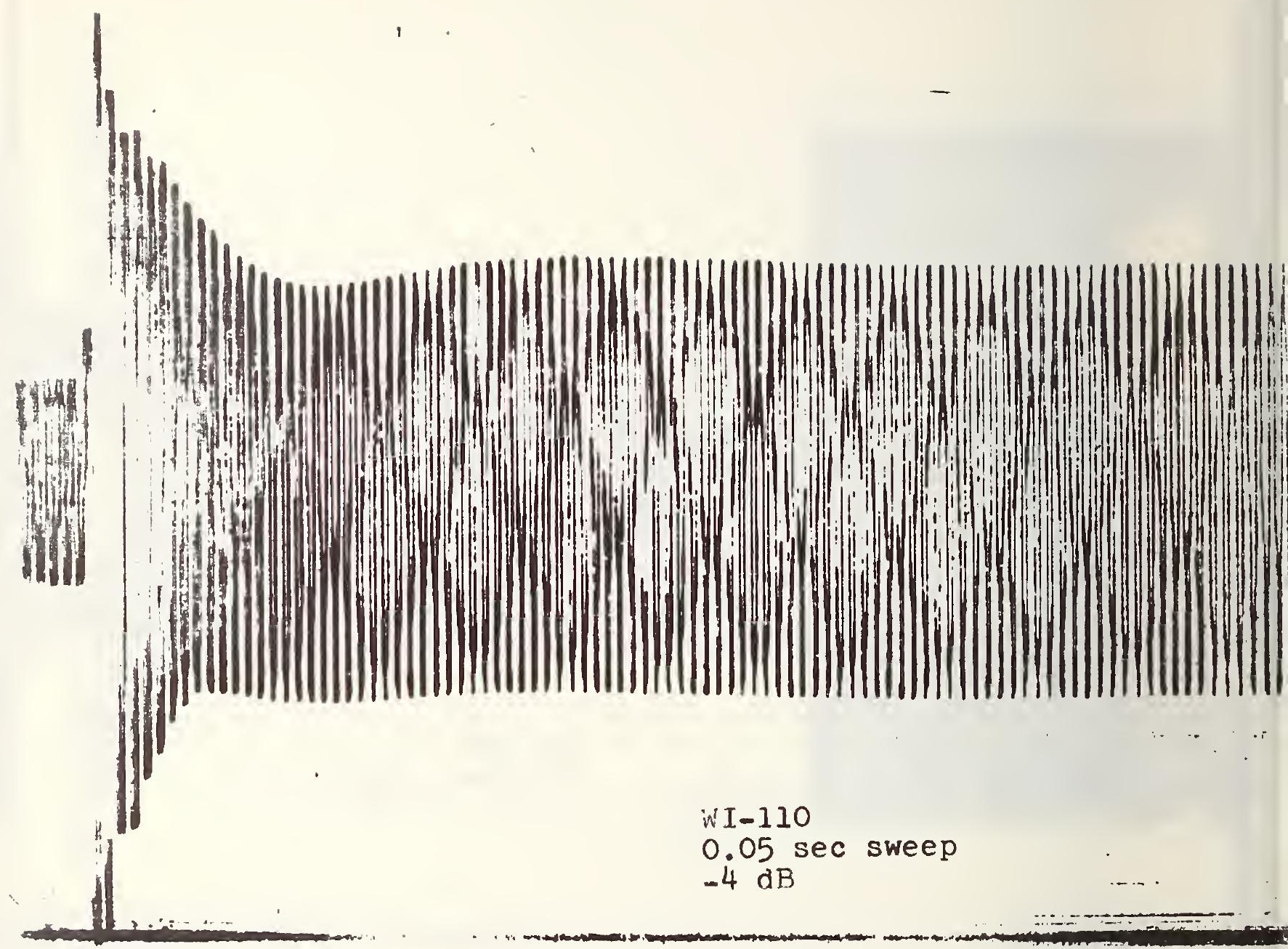




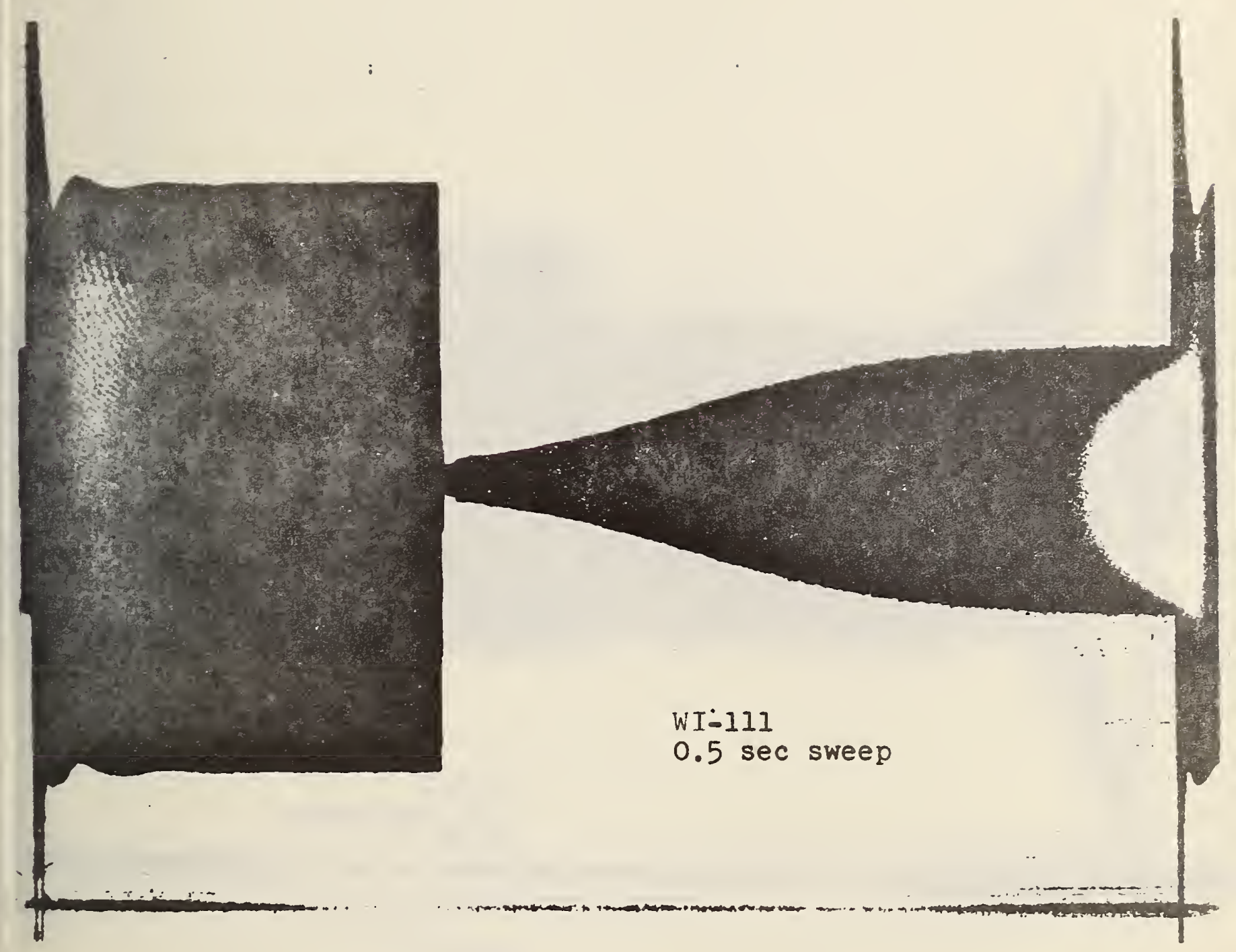




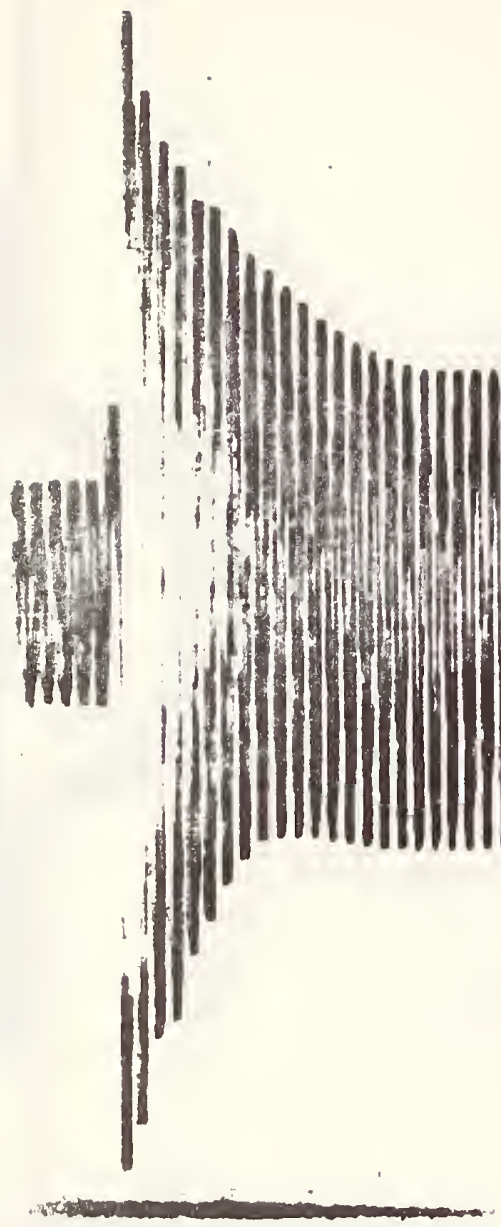

$$
\begin{aligned}
& W I-111 \\
& 0.05 \text { sec sweep } \\
& -5 \mathrm{~dB}
\end{aligned}
$$

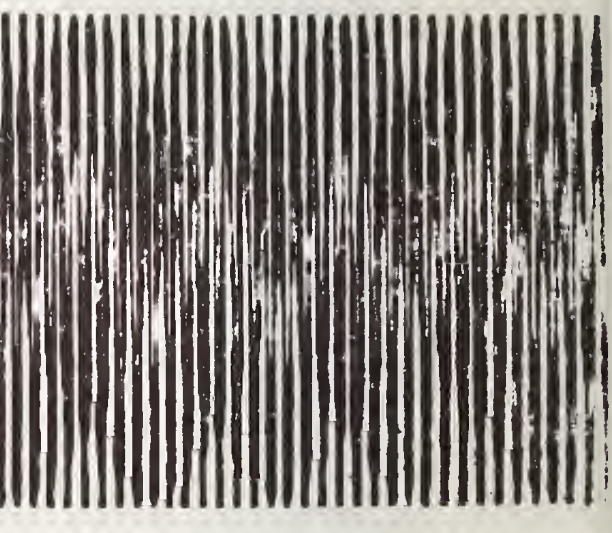




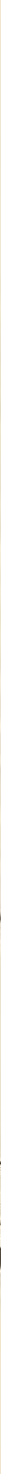




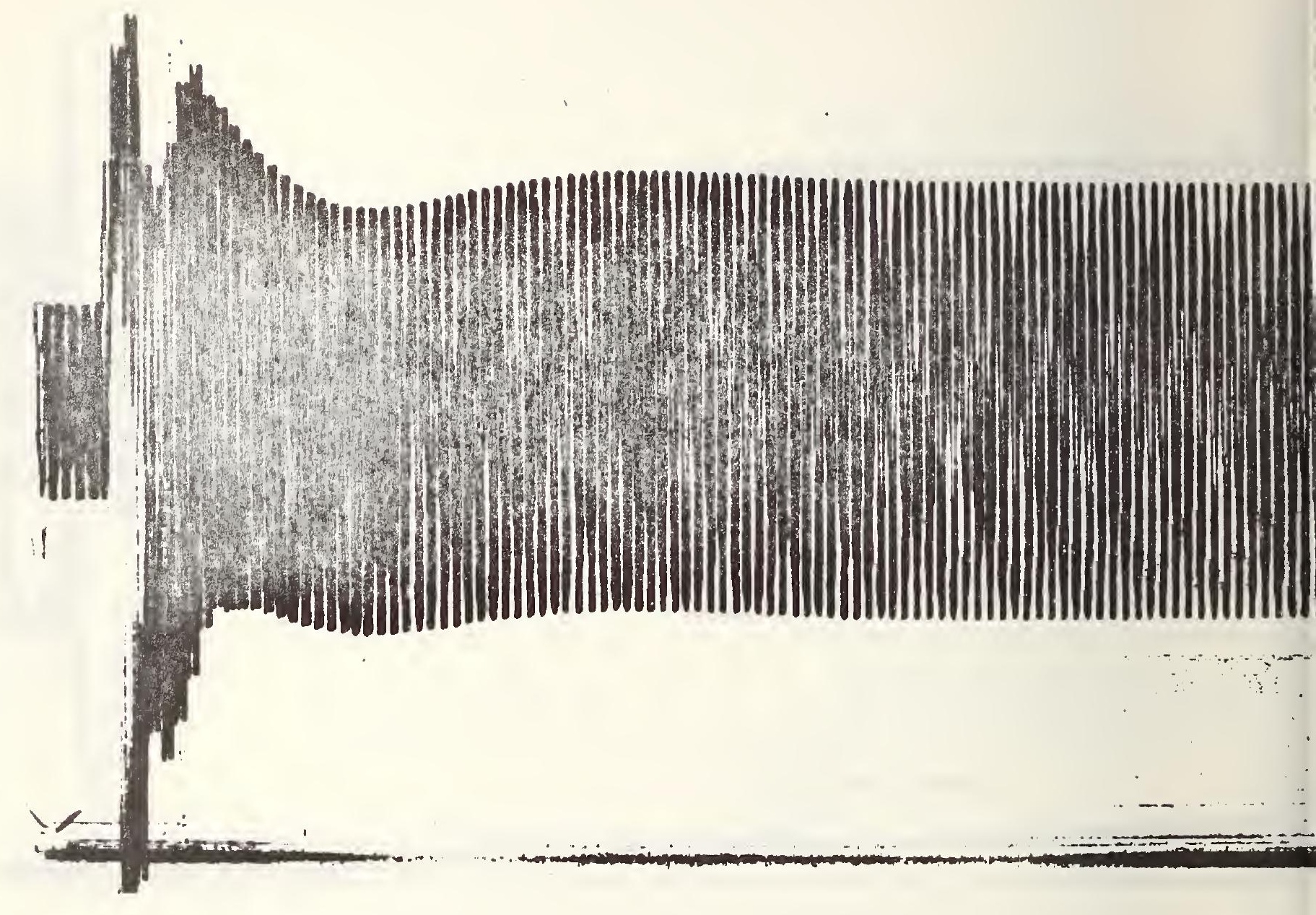

WI-12I

0.05 sec sweep 


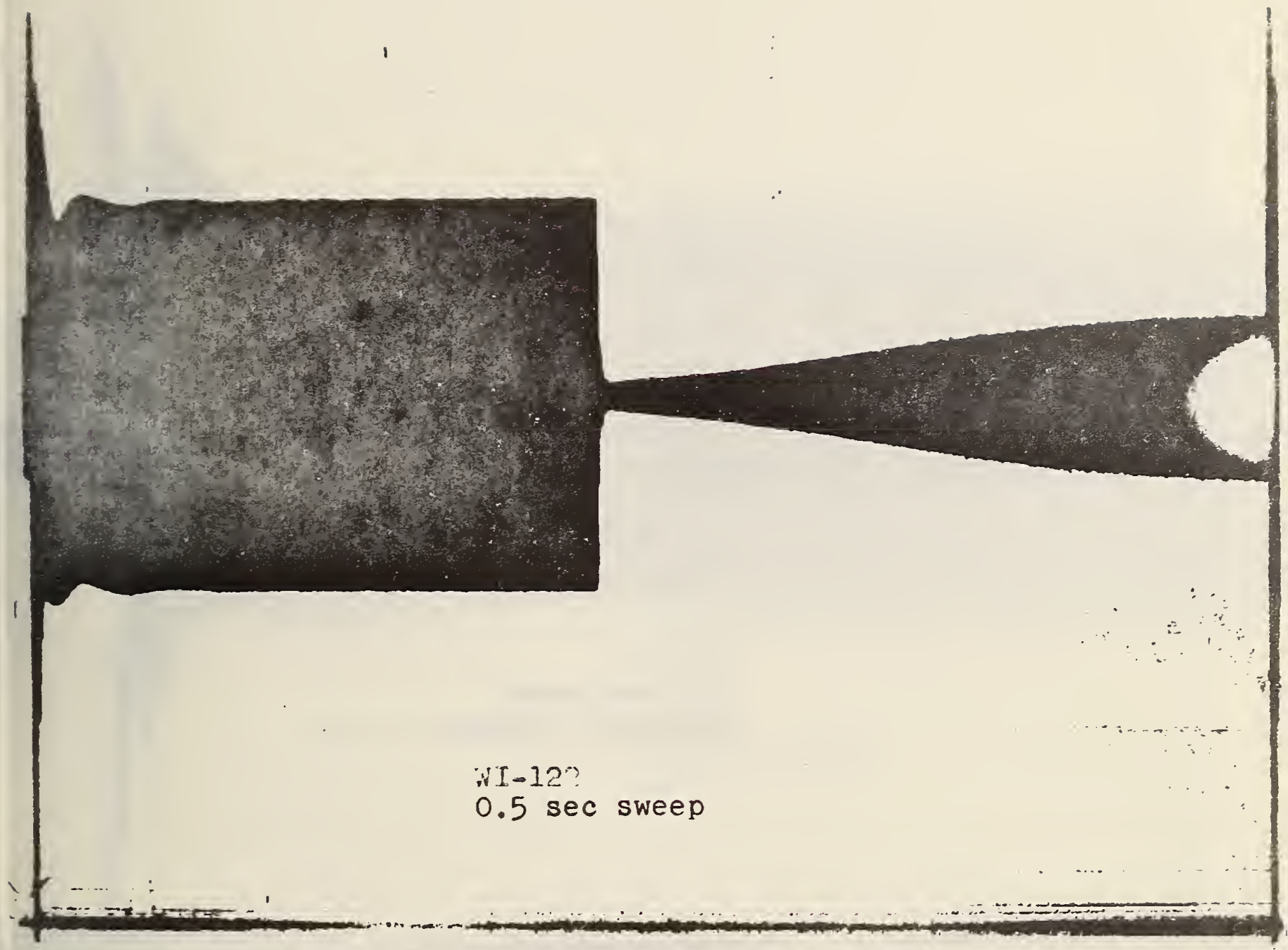




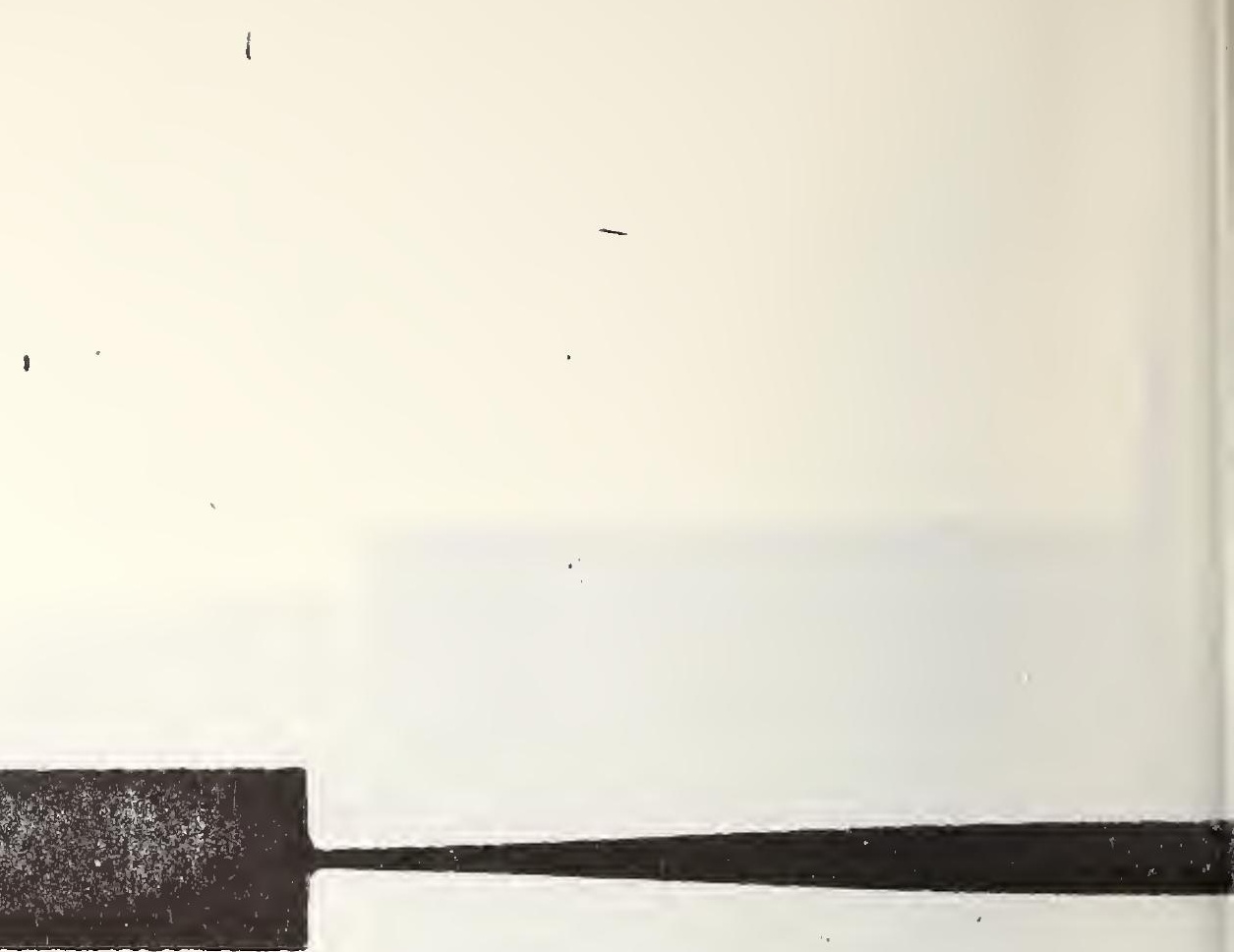

$W . T-122$

0.5 sec sweep

Amplitude reduced $10 \mathrm{~dB}$. 


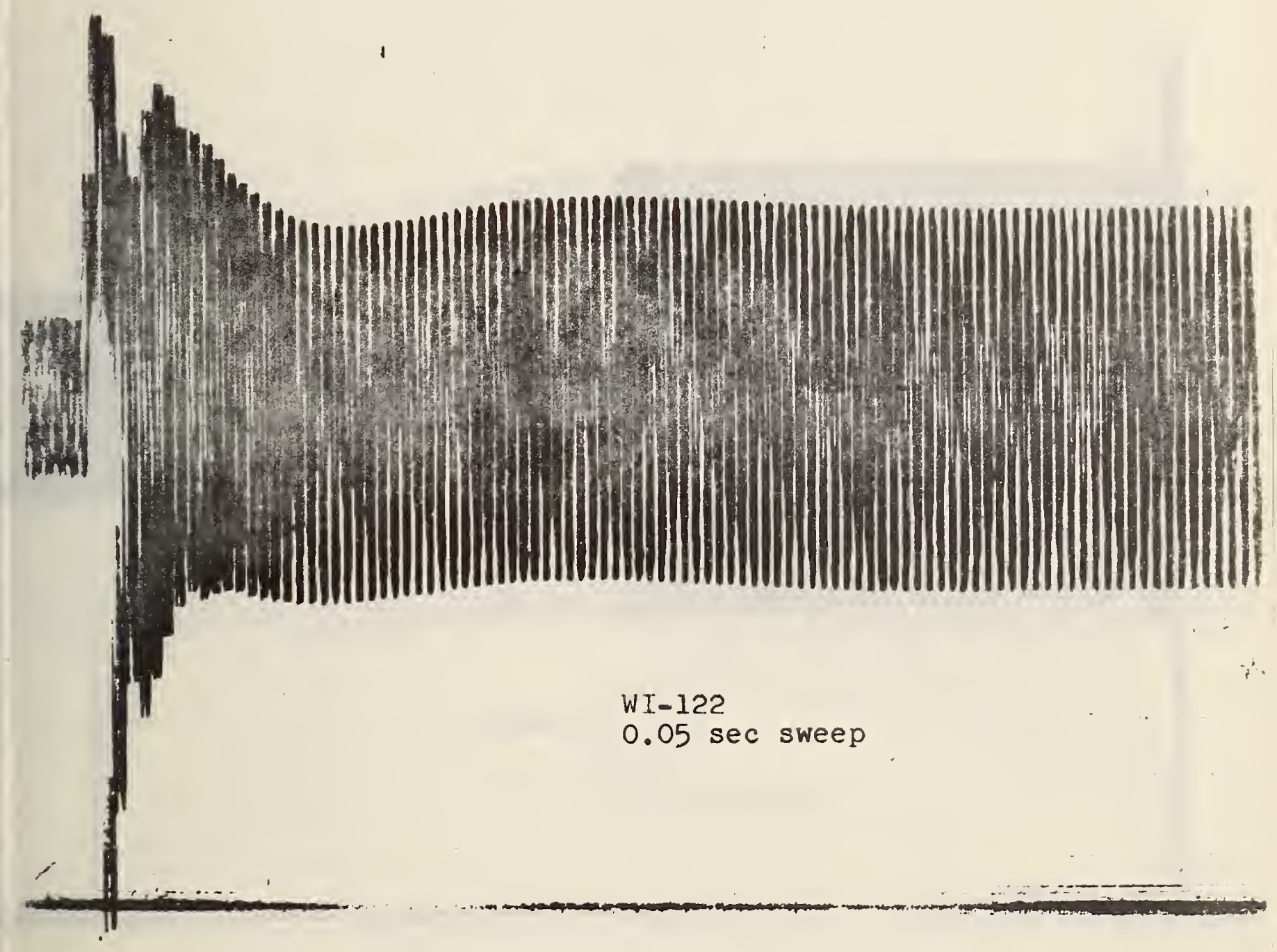




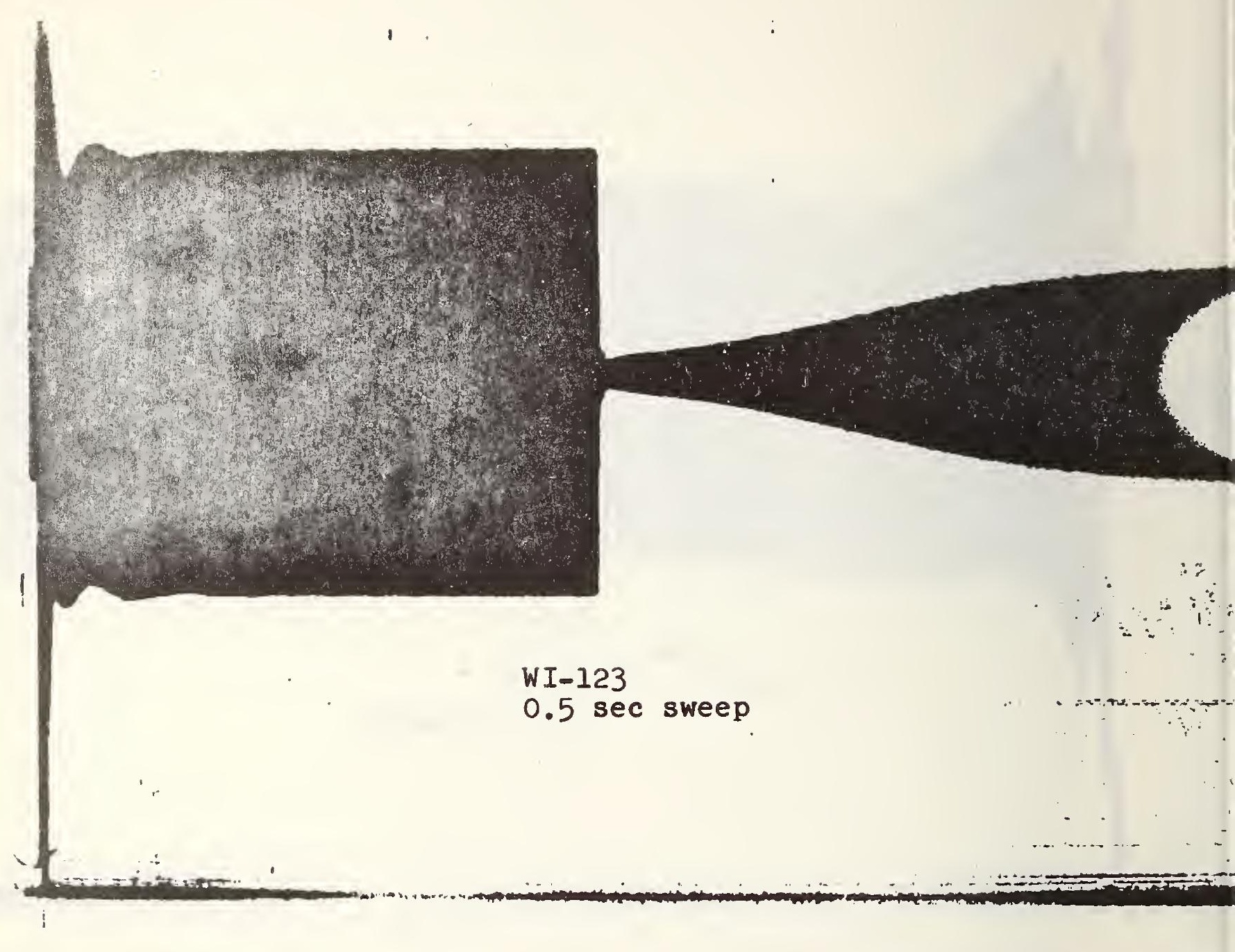

WI-123

$0.5 \mathrm{sec}$ sweep 


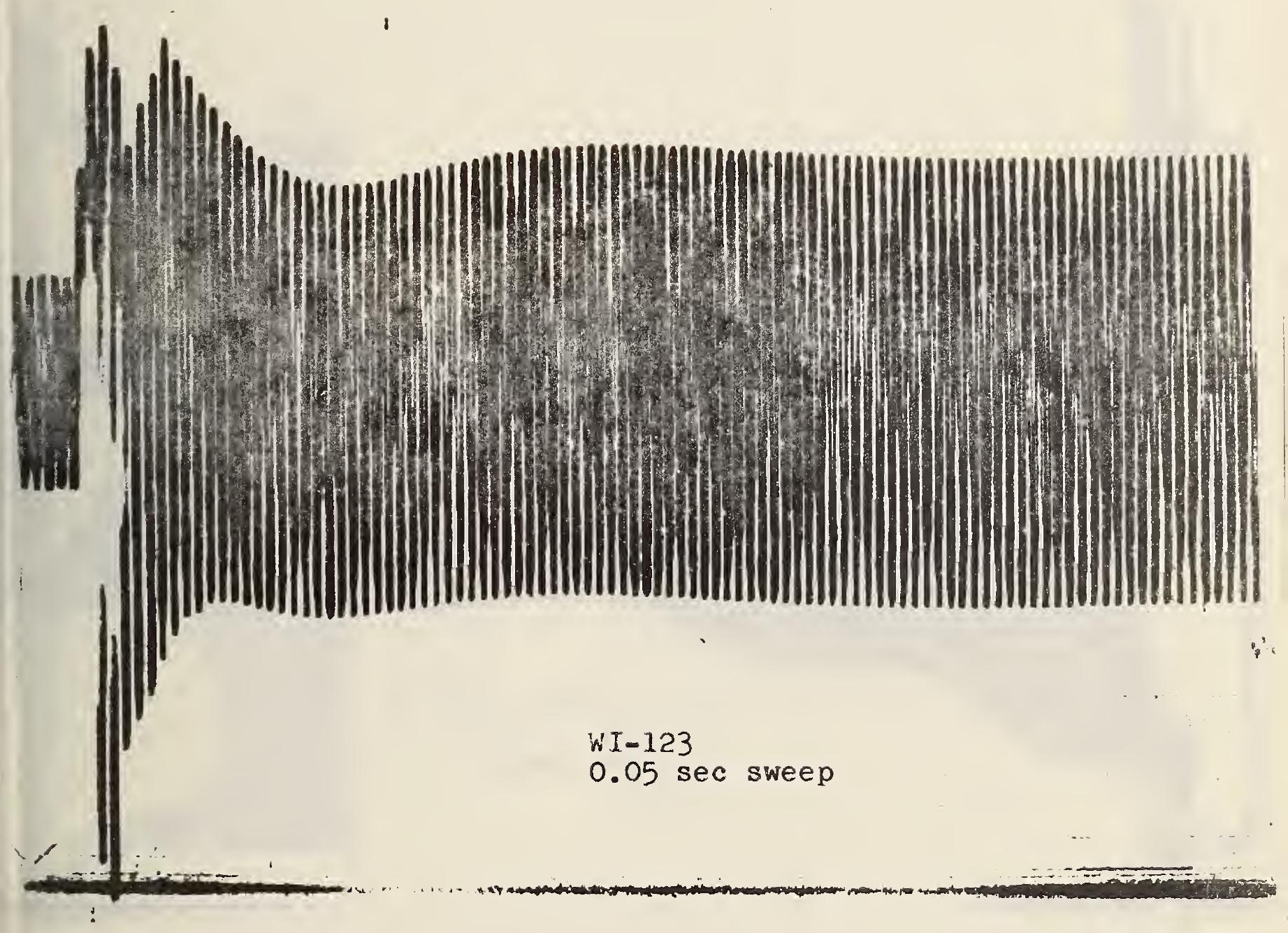




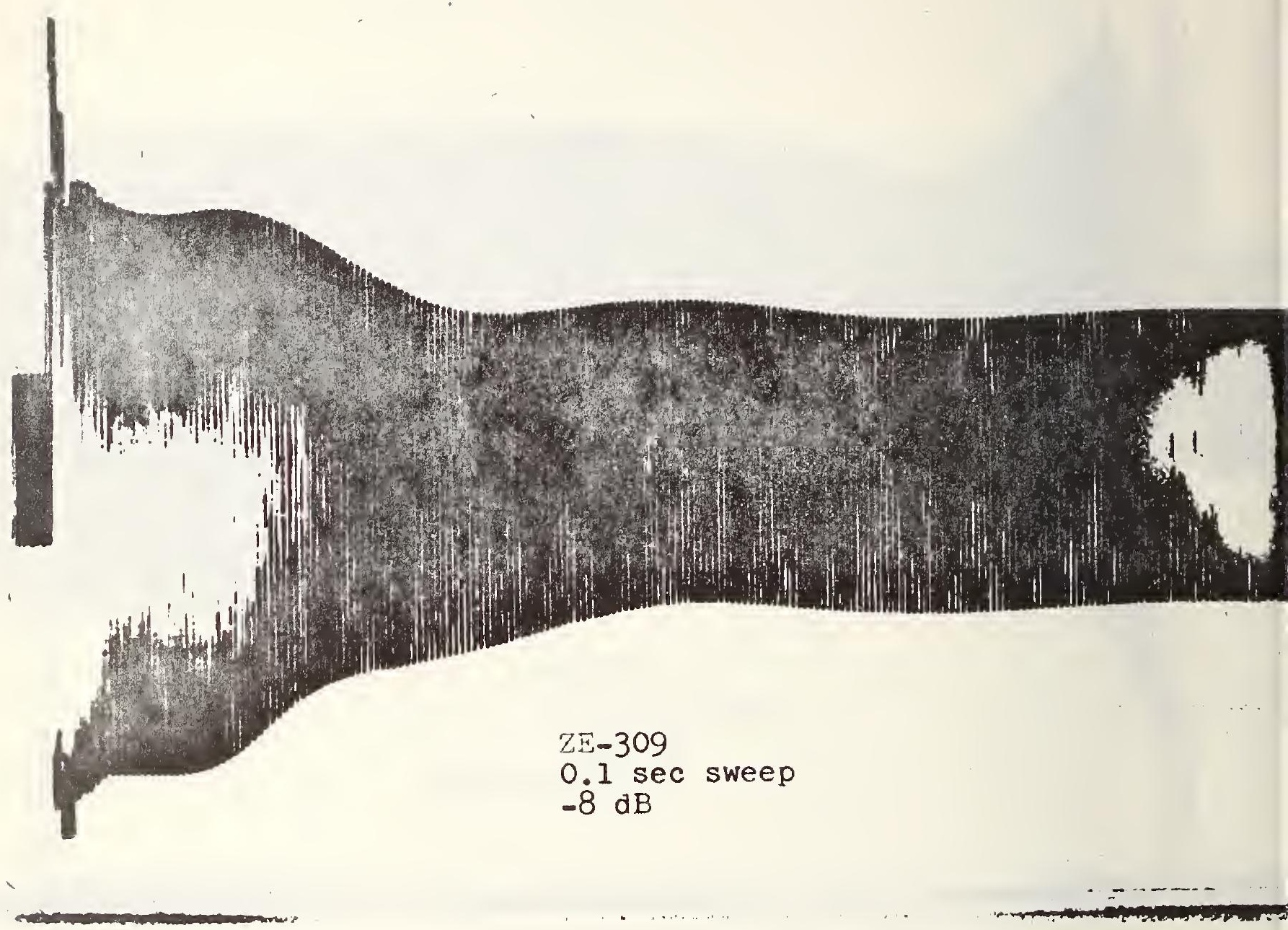




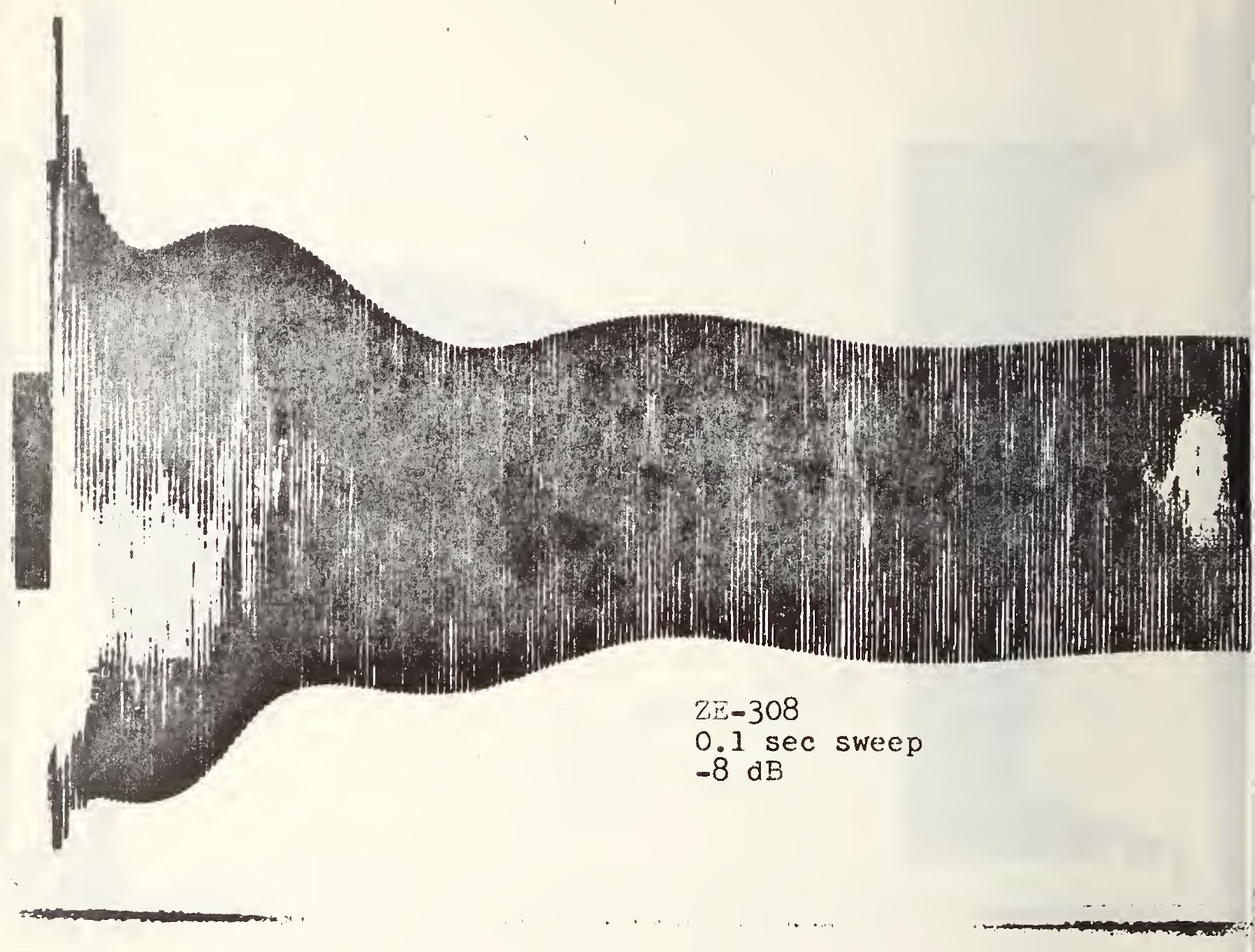




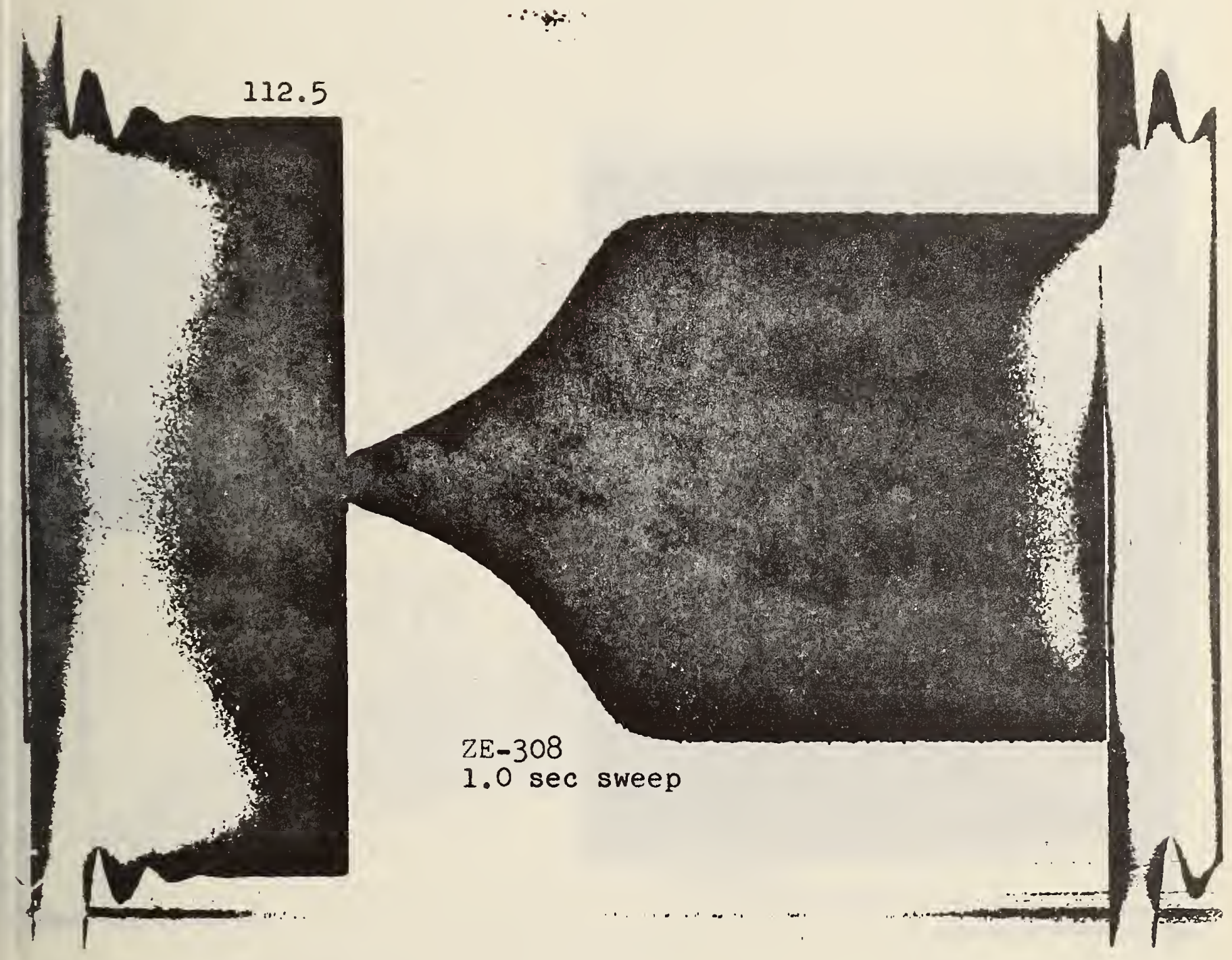




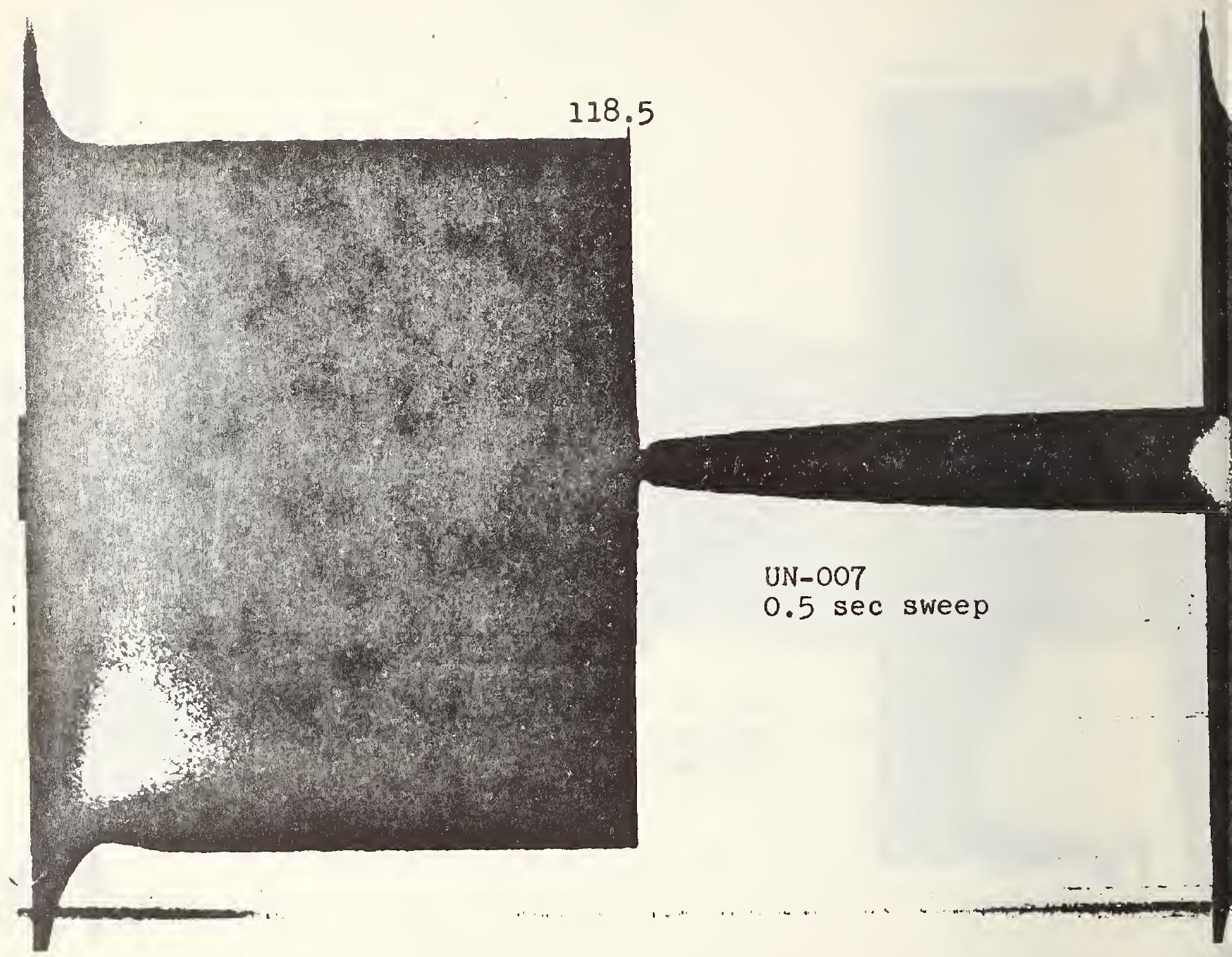




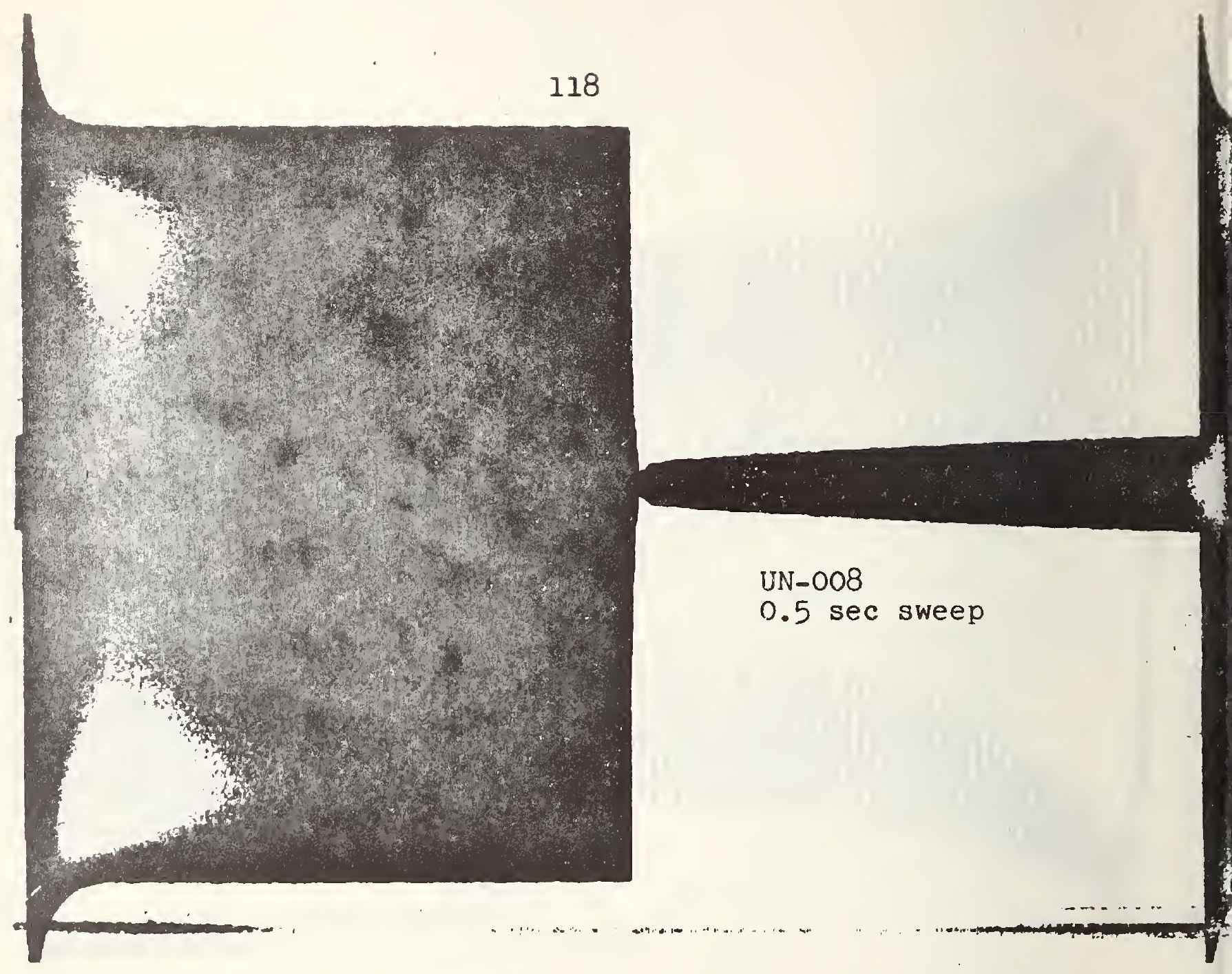



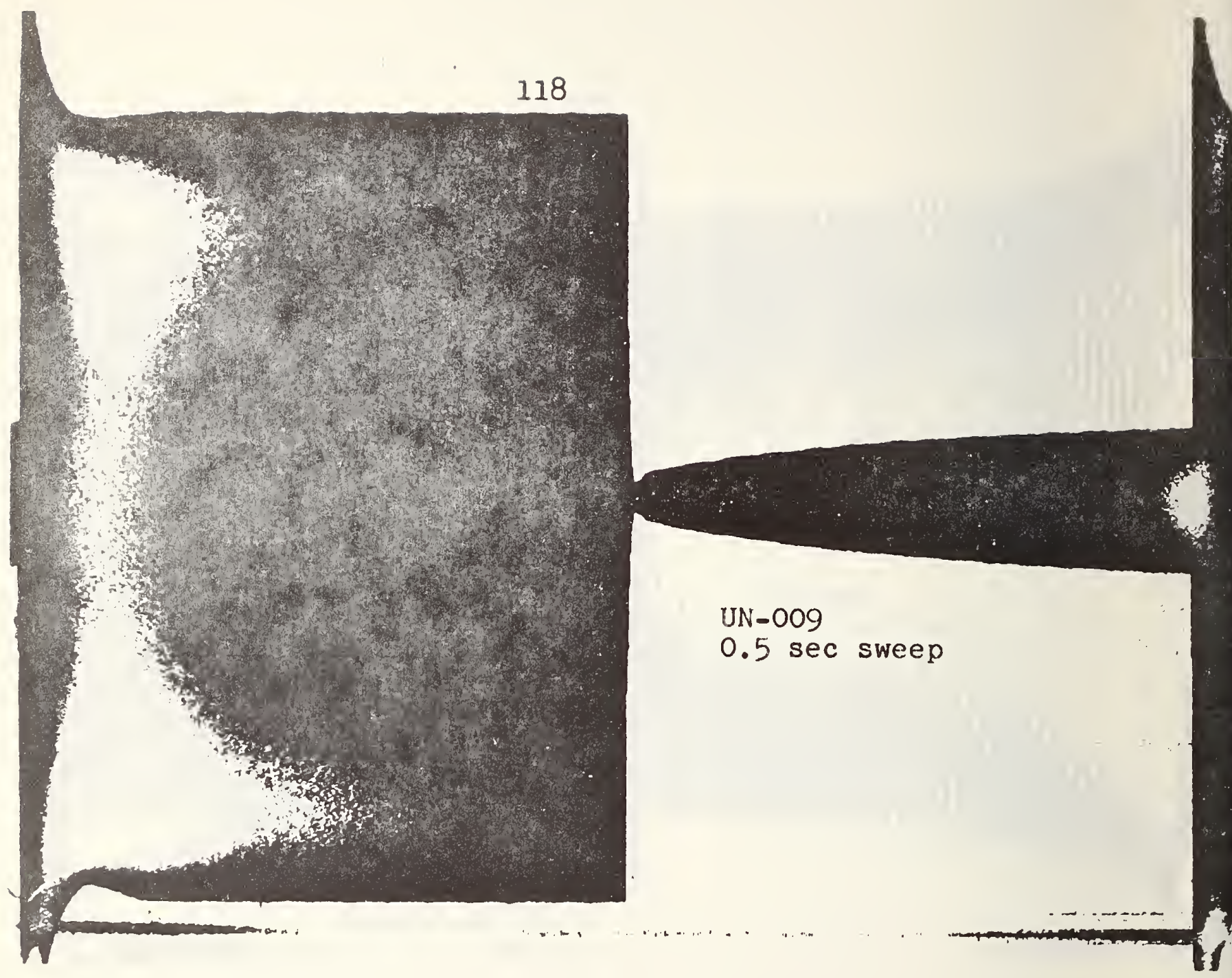


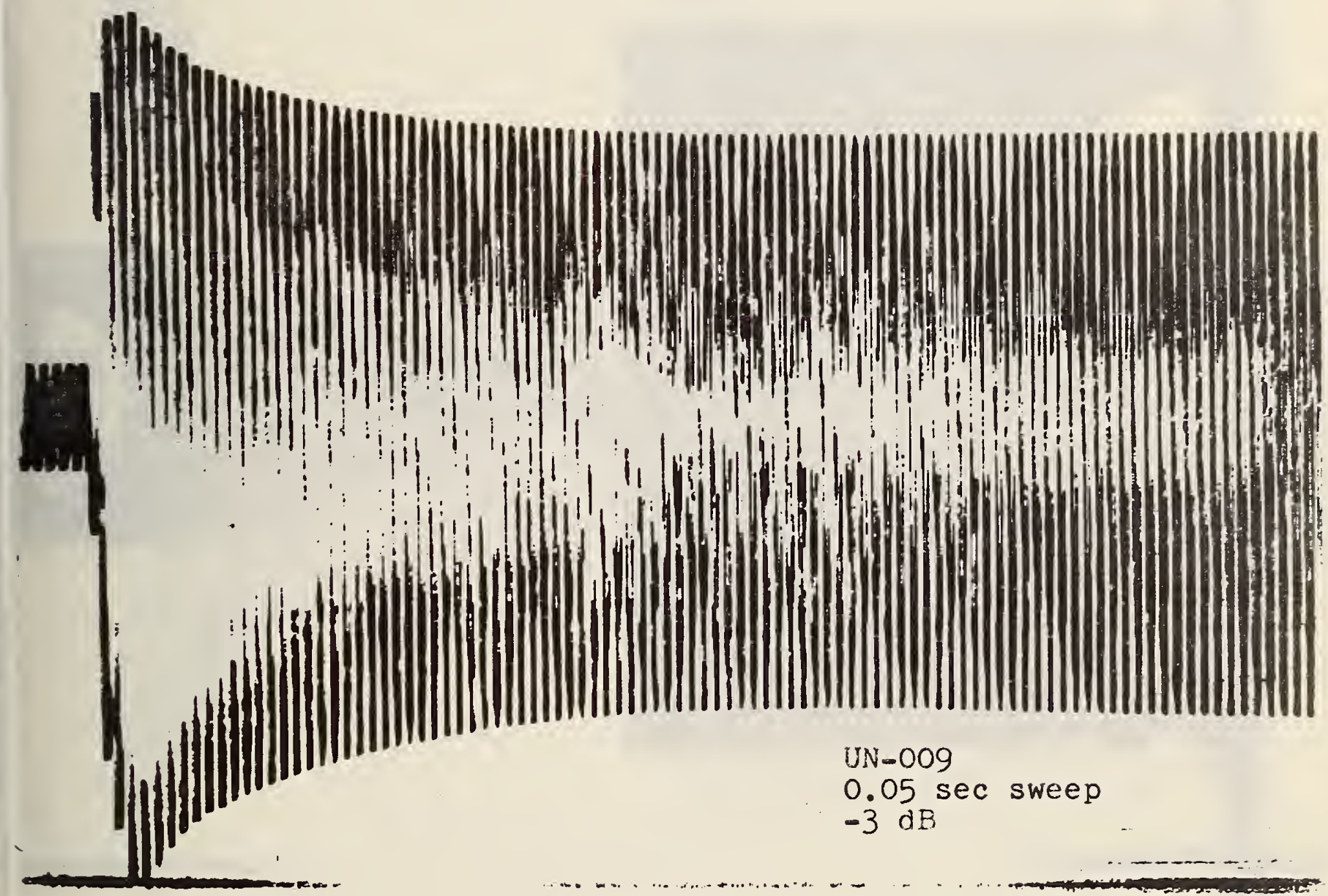




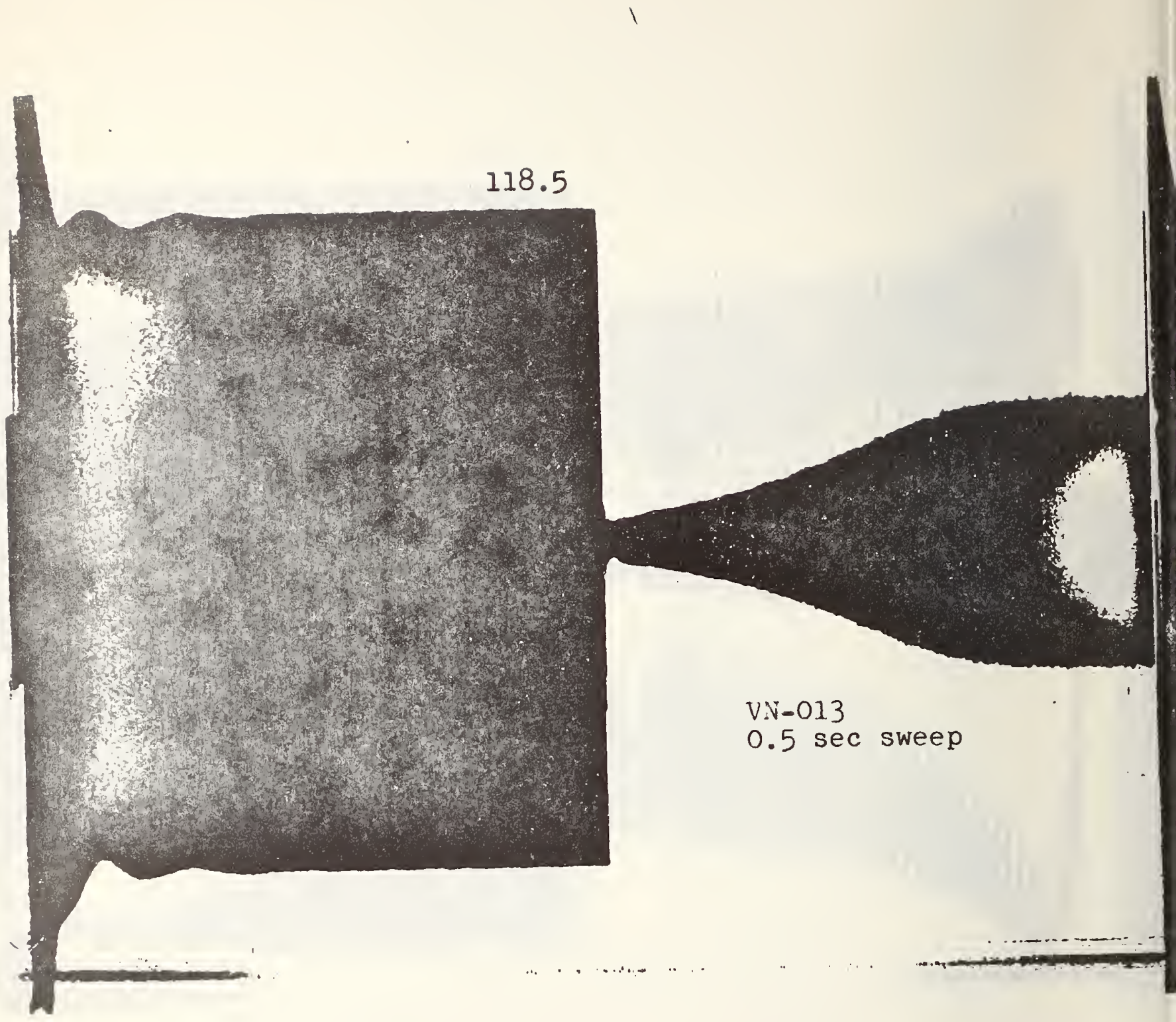




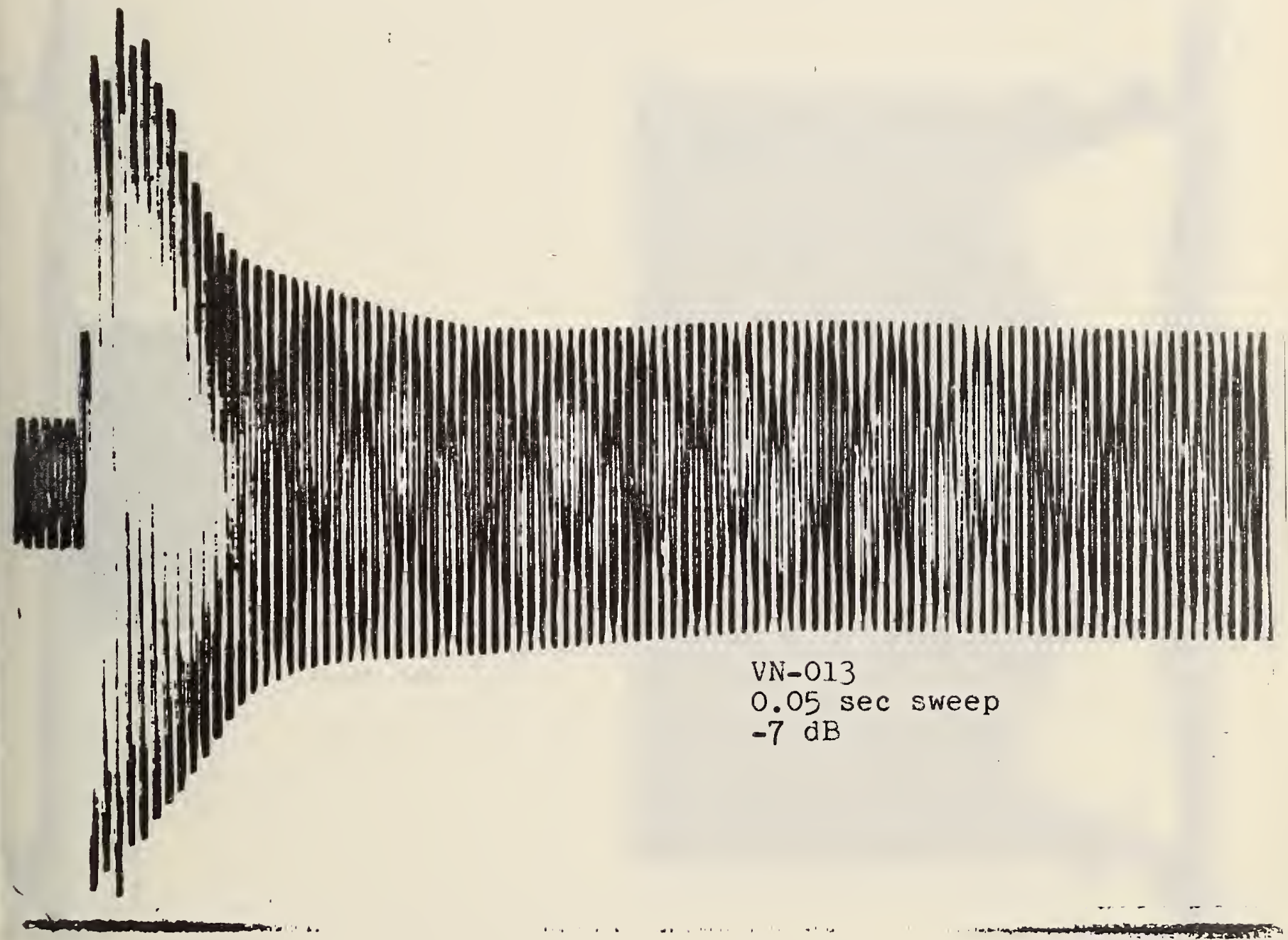




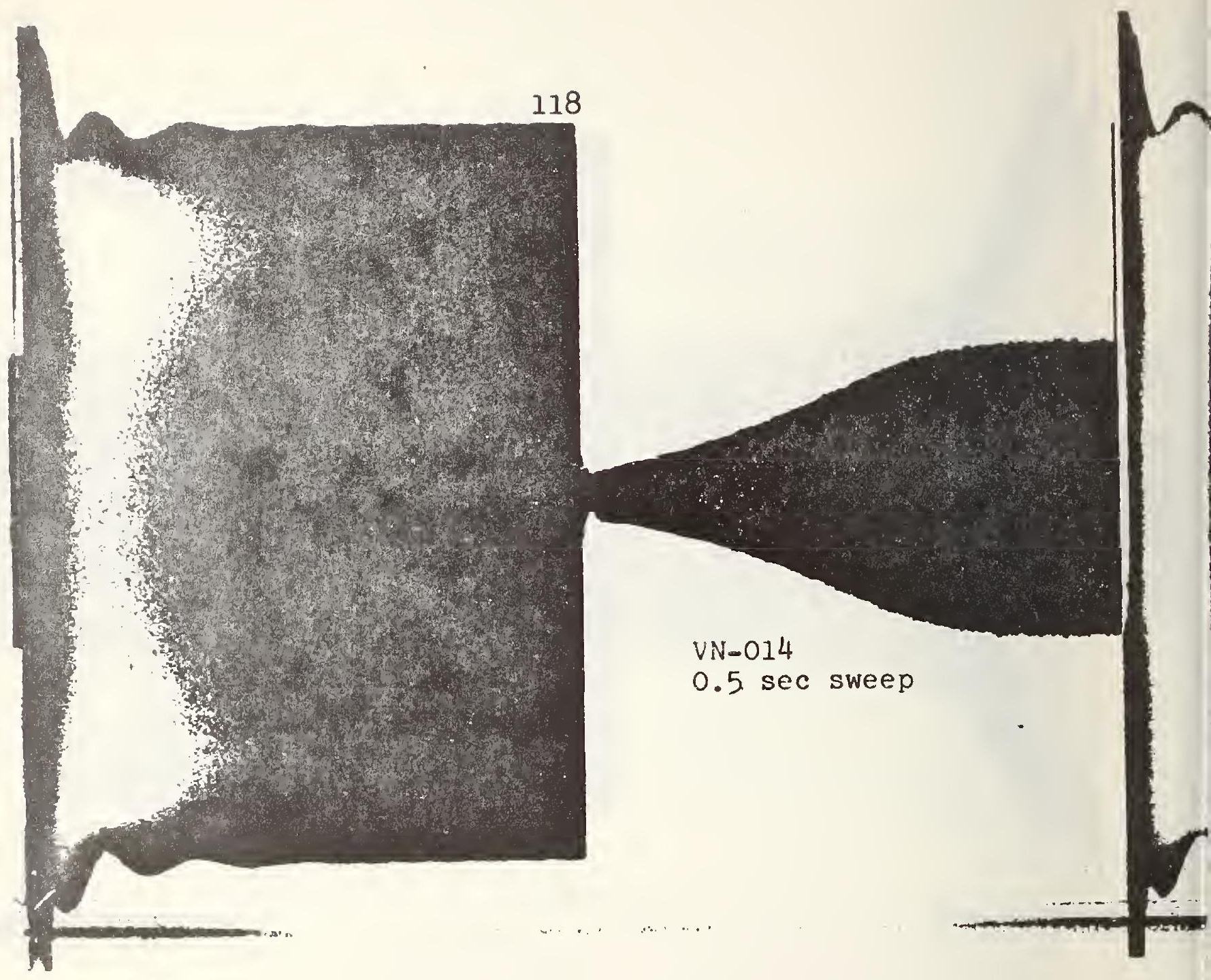




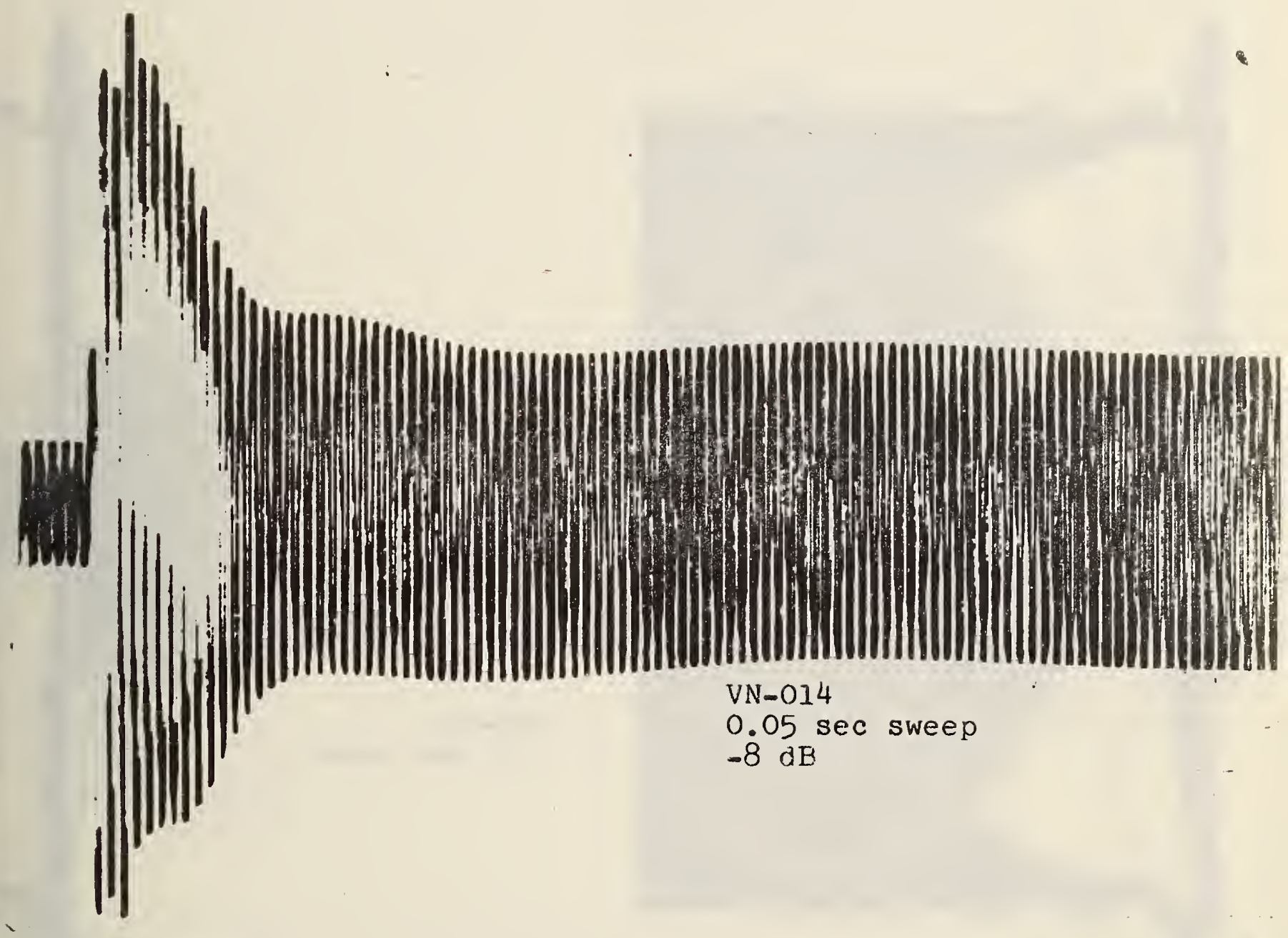

$$
\begin{aligned}
& \mathrm{VN}-014 \\
& 0.05 \text { sec sweep }
\end{aligned}
$$$$
-8 \mathrm{~dB}
$$ 

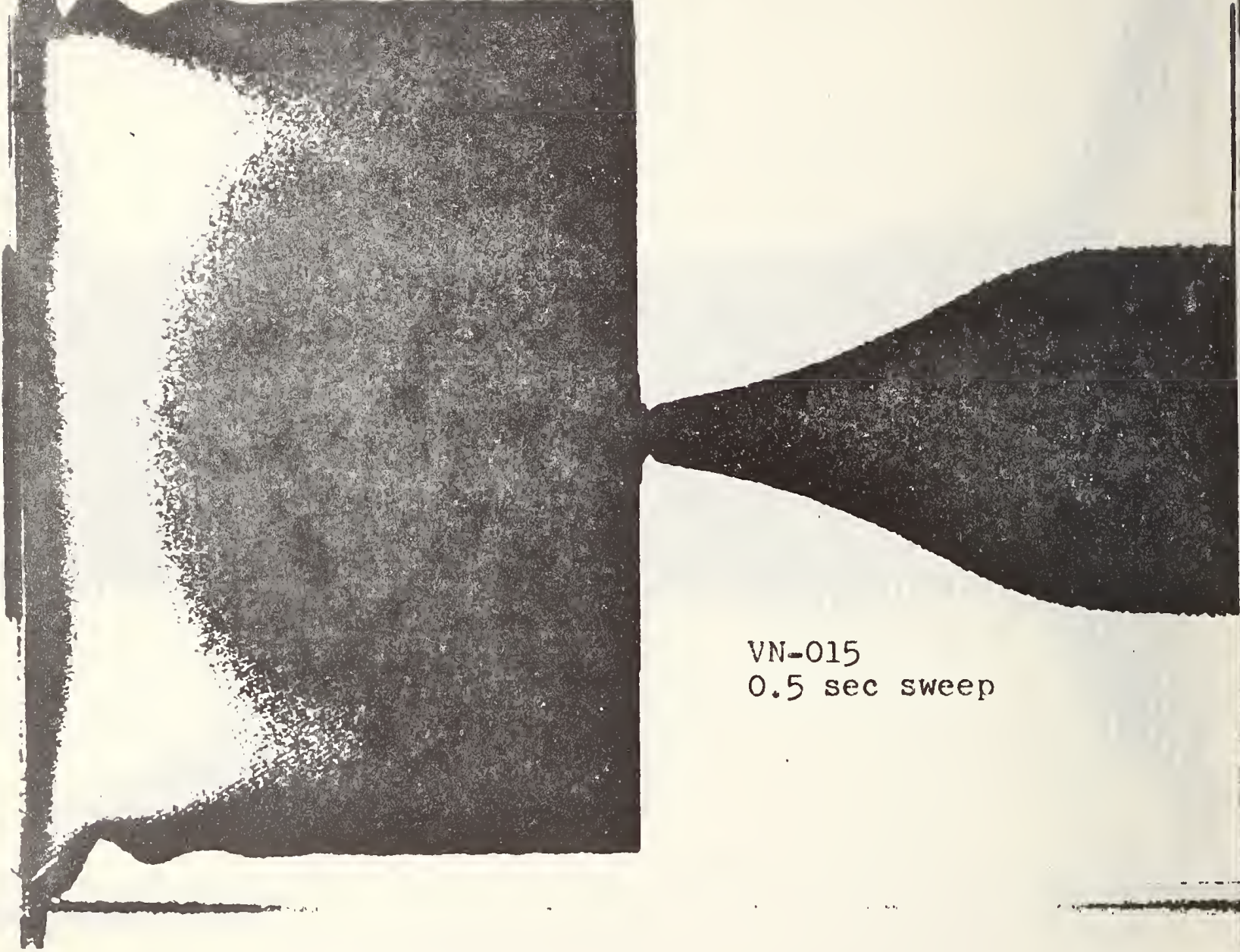

VN-015

0.5 sec sweep 


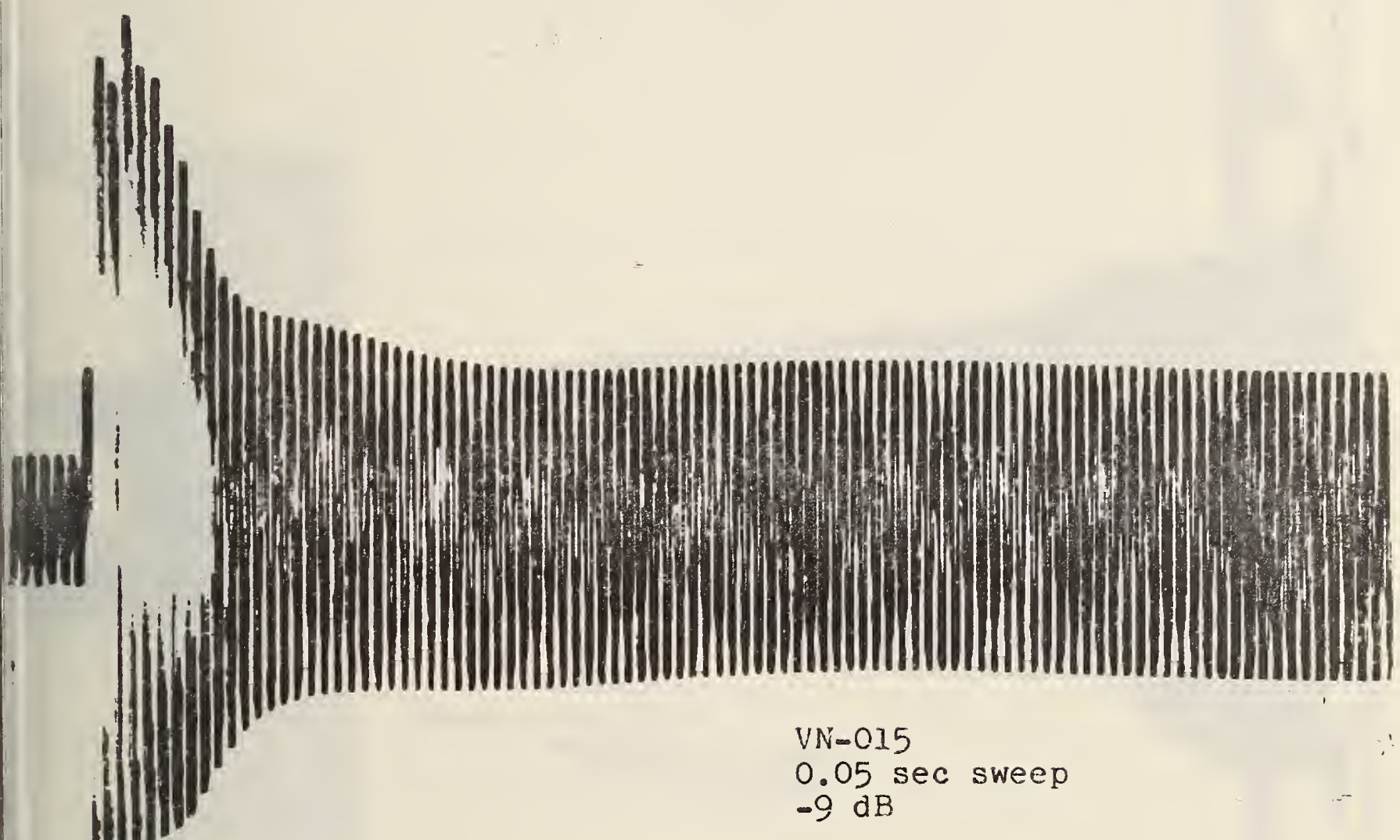

$$
\begin{aligned}
& \text { VN }-015 \\
& 0.05 \text { sec sweep } \\
& -9 \mathrm{~dB}
\end{aligned}
$$




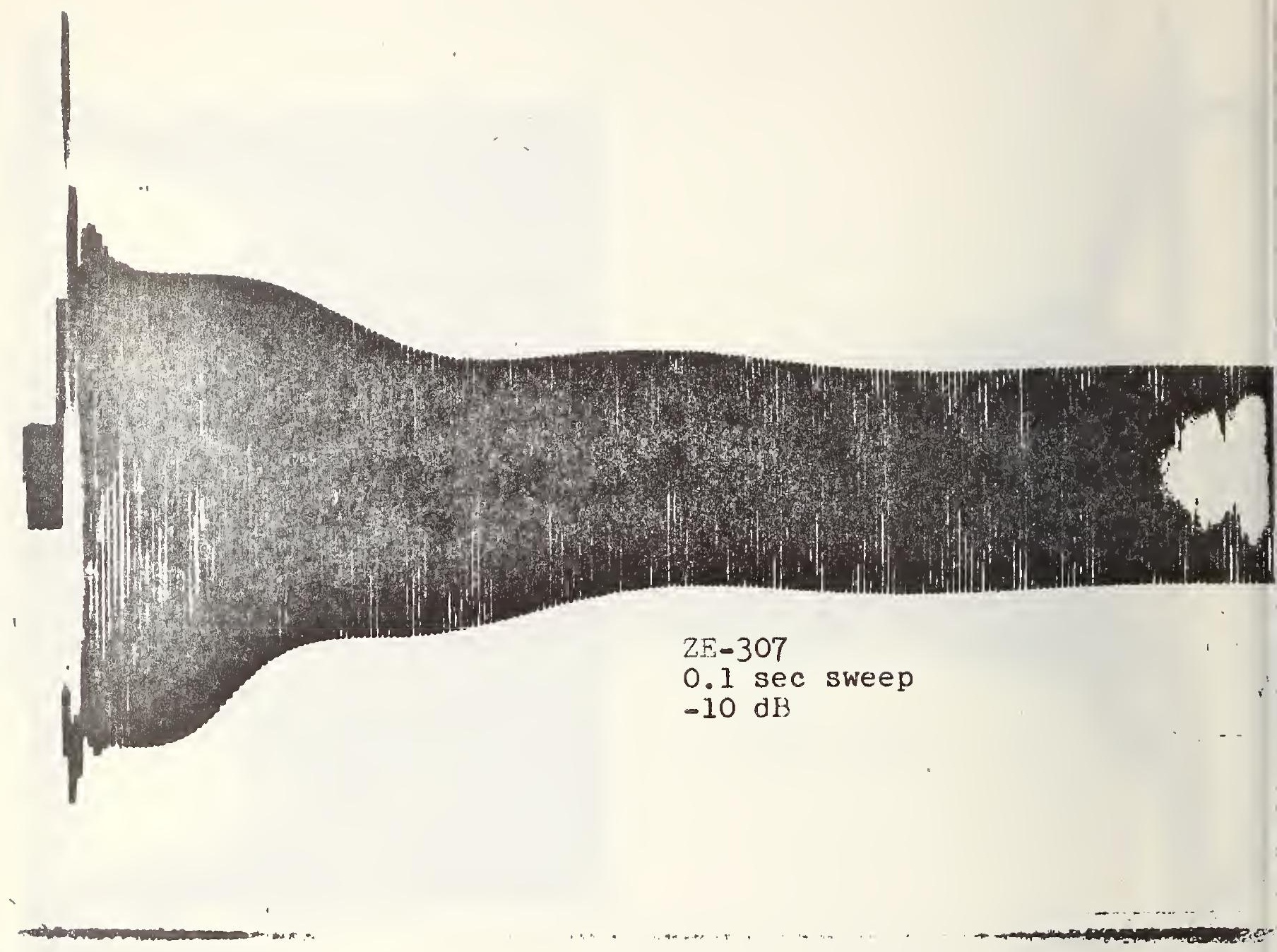


Attack and Release Characteristics of Compression

Hearing Aids

6. Performing Organization Code

200.03

7. AUTHOR(S) Edwin D. Burnett and Martin A. Bassin

9. PERFORMING ORGANIZATION NAME AND ADDRESS

\section{NATIONAL BUREAU OF STANDARDS \\ DEPARTMENT OF COMMERCE \\ WASHINGTON, D.C. 20234}

8. Performing Organ. Report No. NBSIR 76-1179

10. Project/Task/Work Unit No. 2003416

11. Contract/Grant No.

12. Sponsoring Organization Name and Complete Address (Street, City, State, ZIP)

Department of Medicine and Surgery

Veterans Administration

Washington, D.C. 20422

13. Type of Report \& Period Covered Final

14. Sponsoring Agency Code

15. SUPPLEMENTARY NOTES

16. ABSTRACT (A 200-word or less factual summary of most significant information. If document includes a significant bibliography or literature survey, mention it here.)

The attack and release time characteristics of 81 compression hearing aids are presented. The input signal used was that specified in the standard, ANSI S3.22.

17. KEY WORDS (six to twelve entries; alphabetical order; capitalize only the first letter of the first key word unless a proper name; separated by semicolons)

Attack and release characteristics; compression; hearing aids

18. AVAILABILITY

$\square$ For Official Distribution. Do Not Release to NTIS

[ Order From Sup. of Doc., U.S. Government Printing Office Washington, D.C. 20402, SD Cat. No. C13

$x$ Order From National Technical Information Service (NTIS) Springfield, Virginia 22151

\begin{tabular}{|l|c|}
\hline $\begin{array}{l}\text { 19. SECURITY CLASS } \\
\text { (THIS REPURT) }\end{array}$ & 21. NO. OF PAGES \\
UNCL ASSIFIED & 174 \\
\hline $\begin{array}{l}\text { 20. SECURITY CLASS } \\
\text { (THIS PAGE) }\end{array}$ & 22. Price \\
UNCLASSIFIED & $\$ 6.75$ \\
\hline
\end{tabular}

